\title{
THE DISCRETE YET UBIQUITOUS THEOREMS OF CARATHÉODORY, HELLY, SPERNER, TUCKER, AND TVERBERG
}

\author{
JESÚS A. DE LOERA, XAVIER GOAOC, FRÉDÉRIC MEUNIER, \\ AND NABIL H. MUSTAFA
}

\begin{abstract}
We discuss five fundamental results of discrete mathematics: the lemmas of Sperner and Tucker from combinatorial topology and the theorems of Carathéodory, Helly, and Tverberg from combinatorial geometry. We explore their connections and emphasize their broad impact in application areas such as data science, game theory, graph theory, mathematical optimization, computational geometry, etc.
\end{abstract}

\section{Contents}

1. Introduction

1.1. The five theorems at a glance

1.2. Notation and preliminaries

2. Combinatorial topology

2.1. Sperner and Tucker

2.2. Continuous versions

2.3. Generalizations and variations

2.4. Computational considerations

3. Combinatorial convexity

3.1. Carathéodory

3.2. Helly

3.3. Tverberg

3.4. Computational considerations

4. Games and fair division

4.1. Strategic games

4.2. Two fair-division problems: cakes and necklaces 454

5. Graphs

5.1. Chromatic number of graphs

5.2. Colorful independent sets

5.3. Kernels in graphs

6. Optimization

6.1. Linear programming

Received by the editors June 16, 2018.

2010 Mathematics Subject Classification. Primary 52Cxx, 57M99, 90Cxx, 91Axx.

The first author was partially supported by LabEx Bezout grant ANR-10-LABX-58 and also by NSF grant DMS-1522158.

The second author was partially supported by Institut Universitaire de France.

The fourth author was supported by ANR SAGA grant JCJC-14-CE25-0016-01. 
6.2. Integer programming

6.3. LP duality

6.4. Convex optimization

6.5. Sampling approaches

7. Data point sets

7.1. Equipartitioning: the ham sandwich theorem and its relatives 477

7.2. Parametrized partitioning of data via geometric methods 480

7.3. Parametrized partitioning of data via topological methods 484

7.4. Depth of point sets

Acknowledgments

About the authors

References 493

\section{INTRODUCTION}

This article surveys the theory and applications of five elementary theorems. Two of them, due to Sperner and Tucker, are from combinatorial topology and are well known for being the discrete analogues of Brouwer's fixed point theorem and the Borsuk-Ulam theorem. The other three, due to Carathéodory, Helly, and Tverberg, are the pillars of combinatorial convexity. These theorems are between fifty and one hundred years old, which is not very old as far as mathematics goes, but have already produced a closely knit family of results in combinatorial geometry and topology. They have also found spectacular applications in, among other places, mathematical optimization, equilibrium theorems for games, graph theory, fairdivision problems, the theory of geometric algorithms, and data analysis.

The first goal of this paper is to introduce some of the many reformulations and variations of our five theorems and explore how these results fit together. It is convenient to split this presentation into two parts. Sections 2 and 3 discuss the Sperner and Tucker theorems and the Carathéodory, Helly, and Tverberg theorems, respectively. At a coarse level, the former deals with combinatorial topology and the latter deals with combinatorial geometry. In each case, we include a special section on algorithmic aspects of these results relevant later for applications.

The second goal of this survey is to sample some of the many applications of our five theorems. In Sections 4 to 7 we proceed by broad areas and examine examples from game theory and fair division, from graph theory, from optimization, and from geometric data analysis. Some of our illustrations are classical (e.g., Nash equilibria, von Neumann's min-max theorem, linear programming), others are more specialized (e.g., Dol'nikov's colorability defect or the polynomial partitioning technique). We aim to show that our five theorems provide simple proofs of each example. This led us to present some new proofs, for instance for Meshulam's lemma (Section 2) or for the ham sandwich theorem (Section 7).

The research topics that we discuss are vibrant and have already prompted a number of prior surveys [28, 42, 50, 71, 120, 152, 211, 219, 396, but other surveys were focused on a single one of the five theorems or did not cover applications. The important developments that we present here are from the past few years and emphasize both a global view and the value of geometric and topological ideas for modern applied and computational mathematics. This research area abounds with 
open questions, all the more enticing because they can often be stated without much technical apparatus. We made a particular effort to stress some of them.

1.1. The five theorems at a glance. Let us start with a classical rendition of Brouwer's fixed point theorem. If you stand in your favorite Parisian boulangerie holding a map of the city in your hands, then crumple it, squeeze it (without ripping it apart, mind you), and throw it to the ground, some point on the map must have landed right on top of its precise loca-

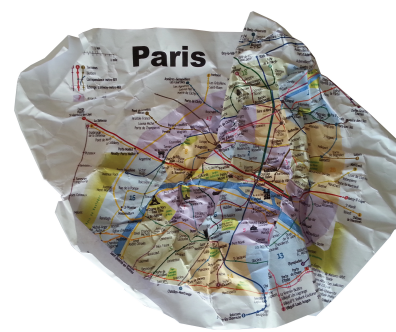
tion. Brouwer's theorem follows from the classical Sperner lemma on the labeling of triangulations (see Figure 11). Surprisingly, all that is needed to prove Sperner's lemma is to understand why a house with an odd number of openings (doors and windows) must have a room with an odd number of openings. This simplicity and its amazing applications attracted the attention of popular newspapers [370] and video sites [214]. Sperner's lemma is one of these five theorems, and we present it in detail in Section 2.1.
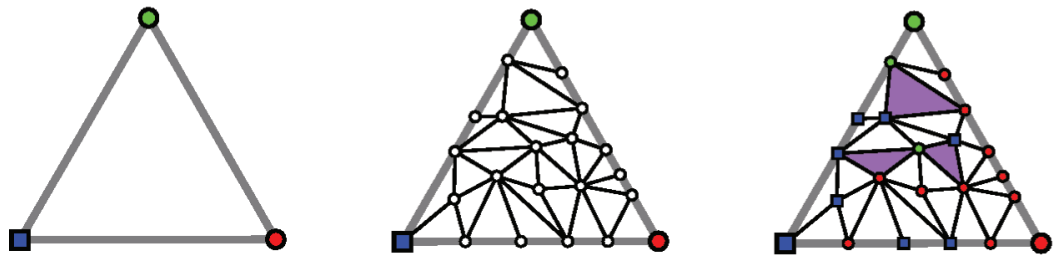

FiguRE 1. Sperner's lemma in the plane. Start with a triangle with vertices colored red, green, blue (left). Subdivide it into smaller triangles that only meet at a common edge or a common vertex (center). Color every new vertex on an edge of the original triangle like either of the vertices of that original edge, and color the remaining vertices arbitrarily (right). At least one of the smaller triangles has vertices with pairwise distinct colors (see the shaded triangles on the right).

In the game of Hex, two players take turns coloring, in black and white, the hexagonal cells of an $11 \times 11$ diamond-shape board (see picture on the right); the opposite sides of the board have matching colors, and the player that manages to connect the two sides of his/her color wins (here, black wins). Since its invention by Hein in 1942, there has never been a draw in Hex. The fact that there is always a winner happens to have a geometric explanation: for any triangulation of the projective plane and any two-coloring of its vertices, one of the color classes spans a noncontractible cycle 361. (To see that this

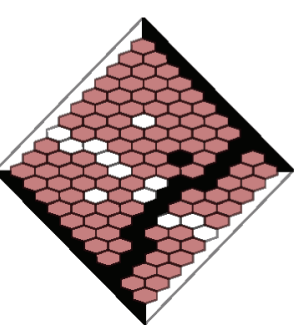
implies the impossibility of a draw in Hex, take the dual of the hexagonal cell decomposition to obtain a triangulation of the diamond, then carefully identify the boundaries to turn that diamond into a projective plane.) This geometric property 

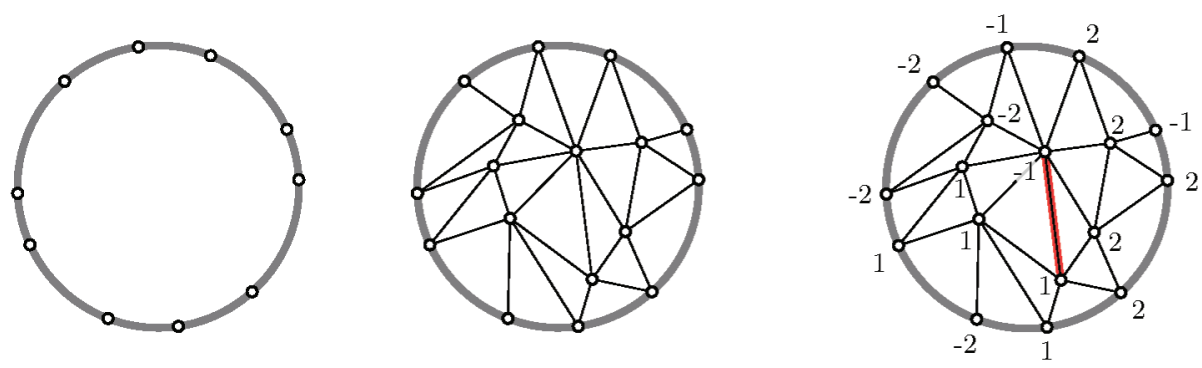

Figure 2. Tucker's lemma in the plane. Start with a symmetric subdivision of the circle (left), and extend it into a triangulation of the disk (center). Label every vertex of the triangulation by $\{-2,-1,1,2\}$ so that antipodal points on the circle get opposite labels (right). There must exist an edge with opposite labels.

is equivalent to the two-dimensional case of Tucker's lemma, whose statement is given in the caption of Figure 2. Tucker's lemma is also discussed in Section 2.1. see in particular the detailed discussion following Proposition 2.2 .

As a matter of fact, Gale [176] proved that the game of Hex cannot end in a draw using Brouwer's fixed point theorem, and Nash 302 proved that for boards of arbitrary size, the first player has a winning strategy. Another application of Tucker's lemma is the ham sandwich theorem, which says that any three finite measures in $\mathbb{R}^{3}$ (such as a piece of bread, a slice of cheese, and a slice of ham for an open-faced sandwich) can be simultaneously bisected by a plane.

Let us now consider finite point sets in the plane. It turns out that any seven points can be partitioned into three

1

0

${ }^{2}$

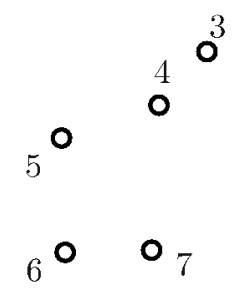
parts so that the triangles, segments, and points that they form have a point in common; for example, the seven points shown at left admit $\{1,7\},\{2,4,6\}$, and $\{3,5\}$ as such a partition. This is the simplest case of Tverberg's theorem. Tverberg's theorem will be discussed at length in Section 3.3. As the number of points grows, so does the number of possible parts in which we can partition the points while assuring all the convex hulls intersect: 10 points allow four parts, 13 points allow five parts, $\ldots$, and in general $3 r-2$ points allow $r$ parts. A similar phenomenon holds in arbitrary dimension: any set of $(r-1)(d+1)+1$ points can be partitioned into $r$ parts whose convex hulls intersect. Coming back to our (uncrumpled) map of Paris, consider the 302 points that represent the subway stations. By Tverberg's theorem, they can be partitioned into 101 parts, so that the corresponding 101 triangles and segments all intersect in a common point $c$. Observe that any line passing through $c$ must leave at least 101 subway stations on either of its (closed) sides. The median of a list of real numbers separates the list in half by sizes. The properties of point $c$ make it an acceptable two-dimensional generalization of the median, but now for the set of subway stations. More generally, the centerpoint theorem, which follows from Tverberg's theorem, asserts that for any finite measure $\mu$ in the plane there is a point $c_{\mu}$ such that $\mu(H) \geq \frac{1}{3} \mu\left(\mathbb{R}^{2}\right)$ for every halfplane $H$ that contains $c_{\mu}$. As 
we will show in Section 7.4, centerpoints are very important objects in applications and have influenced geometry too; e.g., Tverberg himself was motivated to prove his famous theorem (which we discuss in Section 3.3), with the intention of finding an elegant proof of the centerpoint theorem. See the end of his classic paper [376].

In the (fully supervised) classification problem in machine learning, one is given a data set (e.g., images), each with a tag (e.g., indicating whether the image depicts a cat or a car), and one is presented with new data to be tagged. A natural approach is to map the set of data to a set of points in some geometric space and look for a simple separation in the space (e.g., a line or a circle in the plane)

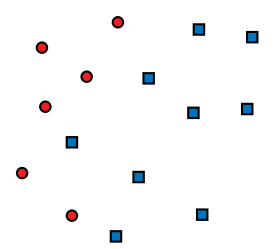
that separates the points with different tags; for instance, perceptron neural networks - some of the basic classifiers - look for a hyperplane best separating the tagged point sets. It is easy to conclude that a separator exists by producing an explicit hyperplane. Kirchberger's theorem states that it is also easy to certify when no line separator exists. In the plane, when no line separator exists, then there must exist a point of one of the colors, say blue, contained in a triangle of the opposite color, red, or a red segment intersecting a blue segment. The set of all lines that define a halfplane containing a given point of $\mathbb{R}^{2}$ defines a convex cone in $\mathbb{R}^{3}$, so Kirchberger essentially reduces

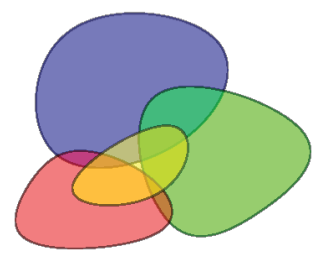
to an intersection property: if a finite family of convex sets in $\mathbb{R}^{d}$ has empty intersection, then some $d+1$ of these sets already have empty intersection. This property is also known as Helly's theorem and is one of our main theorems. The curious reader may check that the centerpoint theorem, discussed above, also follows easily from Helly's theorem. It will be carefully studied in Section 3.2 .

Let us finally turn our attention to the geometry underlying the popular magic squares from ancient China. A magic square is an $n \times n$ square grid of nonnegative real numbers such that the entries along any row, column, and diagonal, all add up to the same value. Look at the four $3 \times 3$ examples on the right. It turns out that any $3 \times 3$ magic square can be written as a linear combination, with

\begin{tabular}{|l|l|l|}
\hline 0 & 2 & 1 \\
\hline 2 & 1 & 0 \\
\hline 1 & 0 & 2 \\
\hline 1 & 2 & 0 \\
\hline 0 & 1 & 2 \\
\hline 2 & 0 & 1 \\
\hline
\end{tabular}

\begin{tabular}{|l|l|l|}
\hline 2 & 0 & 1 \\
\hline 0 & 1 & 2 \\
\hline 1 & 2 & 0 \\
\hline \hline 1 & 0 & 2 \\
\hline 2 & 1 & 0 \\
\hline 0 & 2 & 1 \\
\hline
\end{tabular}
nonnegative coefficients, of only three of these four magic squares! In fact, for any $n$ there exists a finite set $X_{n}$ of $n \times n$ magic squares such that any other $n \times n$ magic square can be written using only $(n-1)^{2}-1$ elements of $X_{n}$. This last statement follows from Carathéodory's theorem, which we will study carefully in Section 3.1: any vector in a cone in $\mathbb{R}^{d}$ is a nonnegative linear combination of extreme rays of the cone, and a linear combination of $k$ elements suffice, where $k$ is the dimension of the cone. Indeed, the set of $n \times n$ magic squares forms a polyhedral cone in a vector space of dimension $(n-1)^{2}-1$. It may come as a surprise that no one knows what $X_{n}$ is for all $n \geq 6$; see $[9$. 


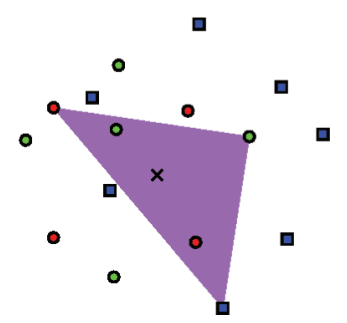

A colorful generalization of Carathéodory's theorem asserts that if three polygons in the plane - one with red vertices, one with green vertices, and one with blue vertices - all contain a given point $p \in \mathbb{R}^{2}$, then there exists a colorful triangle, using a vertex of each color, that also contains $p$. This implies that for the centerpoint $c$ that we constructed earlier for the Parisian subway stations from Tverberg's theorem, at least $\left(\begin{array}{c}101 \\ 3\end{array}\right)$ of the triangles spanned by the subway stations contain $c$. In fact, there is a quantitatively stronger statement given by the first selection lemma. It states that for any set of $n$ points in the plane, there exists a point covered by at least $\frac{2}{9}\left(\begin{array}{l}n \\ 3\end{array}\right)$ of the triangles they span. We will see more about this topic in Section 7.4 .

1.2. Notation and preliminaries. In this subsection we collect notation, terminology, and general basic background on combinatorics, geometry, and topology that will be used in the rest of this survey. The advanced reader may want to skip or move quickly through this section. For a more thorough introduction to the topics listed here, we recommend the classical books and textbooks in combinatorial convexity [52,194, 195, 257] as well as [345, §5.3]. For topological combinatorics and combinatorial aspects of algebraic topology, see [133, 258, 288.

Given $n \in \mathbb{N}$, we write $[n]$ to denote the set $\{1,2, \ldots, n\}$. If $X$ is a set and $k \in \mathbb{N}$, we write $\left(\begin{array}{l}X \\ k\end{array}\right)$ for the set of $k$-element subsets of $X$. The notation $\tilde{O}(\cdot)$ denotes asymptotic notation where we ignore polylogarithmic factors: $f(n)=\widetilde{O}(g(n))$ if there exists $k \in \mathbb{N}$ such that $f(n)=O\left(g(n) \log ^{k} g(n)\right)$.

We denote by $\left(\boldsymbol{e}_{1}, \boldsymbol{e}_{2}, \ldots, \boldsymbol{e}_{d}\right)$ the orthonormal frame of $\mathbb{R}^{d}$. Given two vectors $\boldsymbol{x}$ and $\boldsymbol{y}$ in $\mathbb{R}^{d}$, we write $\boldsymbol{x} \leq \boldsymbol{y}$ to mean that $x_{i} \leq y_{i}$ for $i=1,2, \ldots, d$. We write $\mathbb{B}^{d}=$ $\left\{\boldsymbol{x} \in \mathbb{R}^{d}: \sum_{i=1}^{d} x_{i}^{2} \leq 1\right\}$ for the unit ball in $\mathbb{R}^{d}$ and $\mathbb{S}^{d}=\left\{\boldsymbol{x} \in \mathbb{R}^{d+1}: \sum_{i=1}^{d+1} x_{i}^{2}=1\right\}$ for the unit sphere in $\mathbb{R}^{d+1}$.

1.2.1. Polytopes, simplices, polyhedra, cones. Let $A \subseteq \mathbb{R}^{d}$ be a set. The convex hull of $A$, denoted by $\operatorname{conv}(A)$, is the intersection of all convex sets containing $A$. In other words, $\operatorname{conv}(A)$ is the smallest convex set containing $A$. It is well known that

$$
\operatorname{conv}(A)=\left\{\sum_{i=1}^{n} \gamma_{i} \boldsymbol{a}_{i}: n \in \mathbb{N}, \boldsymbol{a}_{i} \in A, \gamma_{i} \geq 0, \text { and } \gamma_{1}+\cdots+\gamma_{n}=1\right\} .
$$

A polytope is the convex hull of a finite set of points in $\mathbb{R}^{d}$. Here are a few examples. The convex hull of affinely independent points is a simplex; the standard $k$-dimensional simplex $\Delta_{k}$ is $\operatorname{conv}\left(\left\{\boldsymbol{e}_{1}, \ldots, \boldsymbol{e}_{k+1}\right\}\right)$, with $e_{i}$ is the $i$-standard unit vector. The convex hull of $\boldsymbol{e}_{1},-\boldsymbol{e}_{1}, \boldsymbol{e}_{2},-\boldsymbol{e}_{2}, \ldots, \boldsymbol{e}_{k},-\boldsymbol{e}_{k}$ is the $k$-dimensional crosspolytope. The convex hull of all vectors with 0,1 entries is the $d$-dimensional hypercube. A face of a polytope is its intersection with a hyperplane that avoids its relative interior. Faces of dimension 0 are vertices and inclusion-maximal faces are facets. A face of a polytope (resp., simplex) is also a polytope (resp., simplex). There is a face of dimension -1 , the empty set.

A polyhedron is the intersection of finitely many halfspaces in $\mathbb{R}^{d}$. In particular, any polyhedron can be represented as $\left\{\boldsymbol{x} \in \mathbb{R}^{d}: A \boldsymbol{x} \leq \boldsymbol{b}\right\}$, where $A$ is an $n \times d$ matrix and $\boldsymbol{b} \in \mathbb{R}^{n}$. A polyhedral cone is a polyhedron that is closed under addition and 
scaling by a positive constant. In particular, any polyhedral cone can be written as $\left\{\boldsymbol{x} \in \mathbb{R}^{d}: A \boldsymbol{x} \geq \mathbf{0}\right\}$.

The polar of a point $\boldsymbol{x} \in \mathbb{R}^{d} \backslash\{\mathbf{0}\}$ is the halfspace $\left\{\boldsymbol{y} \in \mathbb{R}^{d}: \boldsymbol{x} \cdot \boldsymbol{y} \leq 1\right\}$ (and vice-versa) and the dual of $\boldsymbol{x}$ is the hyperplane $\left\{\boldsymbol{y} \in \mathbb{R}^{d}: \boldsymbol{x} \cdot \boldsymbol{y}=1\right\}$ bounding its polar; we speak of polarity or duality relative to $\boldsymbol{o}$ to represent the polarity or duality after the coordinate system has been translated to have its origin in $\boldsymbol{o}$.

The Weyl-Minkowski theorem asserts that the polytopes are exactly the bounded polyhedra. (This can be proven using polarity arguments.) Polytopes can thus be represented both as convex hulls of finitely many points or as intersections of finitely many halfspaces. To see that these viewpoints are complementary, we invite the reader to prove that projections or intersections of polytopes are polytopes. The Weyl-Minkowski theorem similarly implies that any polyhedral cone also writes as the set of convex combinations of a finite set of rays emanating from the origin. By a theorem of Motzkin et al. 284, every polyhedron $P$ decomposes into the Minkowski sum of a polytope $Q$ and a polyhedral cone $C: P=\{\boldsymbol{x}+\boldsymbol{y}: \boldsymbol{x} \in Q, \boldsymbol{y} \in C\}$.

A polyhedron is pointed if the largest affine subspace it contains is zero dimensional. Any polyhedron can be decomposed into the Minkowski sum of a pointed polyhedron and a vector space.

1.2.2. Simplicial complexes. A geometric simplicial complex is a family of simplices with two properties: the intersection of any two distinct simplices is a face of both of them; and it contains all the faces of every member of the family. Simplicial complexes may also be considered abstractly by only retaining which sets of vertices span a simplex. Formally, an abstract simplicial complex $\mathrm{K}$ is a family of finite subsets (the faces) of some ground set (the vertices) that is closed under taking subsets: if $\sigma \in \mathrm{K}$ and $\tau \subseteq \sigma$, then $\tau \in \mathrm{K}$. We write $\sigma_{n}$ for the abstract $n$-dimensional simplex consisting of all subsets of $[n+1]$. A facet of a simplicial complex is an inclusion-maximal face.

Let us stress that the meaning of the word face (or facet) depends on the context and can denote a polytope (for polytopes), a simplex (for simplices), or a set of vertices (for abstract simplicial complexes). In particular, we consider $\Delta_{n}$ to be a polytope, so it has $n+1$ faces of maximal dimension $n-1$; in contrast, $\sigma_{n}$ has a single face of maximal dimension $n$.

Given a geometric simplicial complex $\mathrm{K}$, we let $|\mathrm{K}|$ denote the underlying topological space, that is $|\mathrm{K}|=\bigcup_{\sigma \in \mathrm{K}} \sigma$. If $\mathrm{L}$ is the abstract simplicial complex obtained from $\mathrm{K}$, we say that $\mathrm{K}$ is a geometric realization of $\mathrm{L}$ and put $|\mathrm{L}|=|\mathrm{K}|$. (The reader can check that all this is well-defined up to homeomorphism.) A triangulation of a topological space $X$ is a (geometric or abstract) simplicial complex whose underlying topological space is homeomorphic to $X$.

1.2.3. Homology. We will use some basic notions of homology, mostly simplicial homology over $\mathbb{Z}$ or $\mathbb{Z}_{q}=\mathbb{Z} / q \mathbb{Z}$. To allow readers unacquainted with homology to appreciate at least our simplest examples, we recall here the basic definitions. An important idea in homology theory is that topological spaces can be studied by associating to them some groups, called homology groups. These groups can be defined geometrically (in singular homology) or combinatorially (in simplicial homology) from a triangulation of the space. In the cases that we consider, these approaches produce isomorphic groups, and we mostly work with simplicial homology. 
Given a simplicial complex $\mathrm{K}$, we denote by $C_{i}\left(\mathrm{~K}, \mathbb{Z}_{2}\right)$ the set of finite formal sums of $i$-dimensional faces of $\mathrm{K}$, and $C \bullet\left(\mathrm{K}, \mathbb{Z}_{2}\right)=\bigoplus_{i=0}^{\infty} C_{i}\left(\mathrm{~K}, \mathbb{Z}_{2}\right)$ is the chain complex of $K$. The map that sends every $i$-face of $K$ to the formal sum, with coefficients in $\mathbb{Z}_{2}$, of its $(i-1)$-faces extends linearly, over $\mathbb{Z}_{2}$, to a map $\partial_{i}: C_{i}\left(\mathrm{~K}, \mathbb{Z}_{2}\right) \rightarrow C_{i-1}\left(\mathrm{~K}, \mathbb{Z}_{2}\right)$. Notice that $C_{\bullet}\left(\mathrm{K}, \mathbb{Z}_{2}\right)$ has an additive group structure and that the sum of the $\partial_{i}$ is a morphism from $C_{\bullet}\left(\mathrm{K}, \mathbb{Z}_{2}\right)$ into itself; this morphism is called the boundary map of $C_{\bullet}\left(\mathrm{K}, \mathbb{Z}_{2}\right)$. Note that with $\mathbb{Z}_{2}$-coefficients finite formal sums are simply subsets and the boundary operator maps to proper subsets that appear an odd number of times. It turns out that $\partial_{i-1} \circ \partial_{i}=0$, so we can define the ith homology group of $\mathrm{K}$ over $\mathbb{Z}_{2}$ coefficients as the quotient group $H_{i}\left(\mathrm{~K}, \mathbb{Z}_{2}\right)=\operatorname{ker} \partial_{i} / \operatorname{im} \partial_{i+1}$.

Intuitively, the rank of $H_{i}\left(\mathrm{~K}, \mathbb{Z}_{2}\right)$ relates to the number of independent holes of dimension $i$ in $|\mathrm{K}|$; for example, the rank of $H_{0}\left(\mathrm{~K}, \mathbb{Z}_{2}\right)$ counts the number of connected components of $|\mathrm{K}|$. In particular, if $\mathrm{K}$ is a single vertex, then all its homology groups are trivial except the 0 th one. The reduced homology groups $\widetilde{H}_{i}$ modify slightly $H_{0}$ so that it is trivial for connected sets; in dimension $i \geq 1$, homology groups and reduced homology groups coincide, and $\widetilde{H}_{-1}$ is defined as 0 for nonempty complexes. Going from $\mathbb{Z}_{2}$ to other coefficient groups involves only one technical complication: the definition of $\partial_{i}$ involves some sign bookkeeping, so as to ensure that $\partial_{i-1} \circ \partial_{i}=0$; see [288] for details.

\section{Combinatorial topology}

From combinatorial topology, we will focus on two results about labeled or colored triangulations of simplicial complexes - Sperner's lemma and Tucker's lemma. The importance of these two lemmas owes much to their special position at the crossroads of topology and combinatorics. As we alluded in the introduction, Sperner's and Tucker's lemmas are the combinatorial equivalent versions to the famous topological theorems of Brouwer and of Borsuk and Ulam, respectively. Their combinatorial nature makes them particularly well-suited for computations and applications too.

Combinatorial structures have been used in algebraic topology since Poincaré's foundational analysis situs, so it is not surprising that some topological questions may be studied by combinatorial methods. The lemmas of Sperner and Tucker are well-known for offering an elementary access, via labelings of combinatorial structures, to important results in topology, such as the theorems of Brouwer and of Borsuk and Ulam.

It is perhaps less obvious that some combinatorial problems may be studied by topological methods. A seminal example of topological methods applied to combinatorics was the use by L. Lovász of the Borsuk-Ulam theorem to settle a conjecture of Kneser on the chromatic number of certain graphs (see Section 5). His paper opened up the application of topological methods in combinatorics that are now common tools. These techniques appear in several books [133, 258, and surveys 62,225$]$. In many cases the topological methods hinge on the theorems of Brouwer or Borsuk and Ulam; as we discuss in the application sections, on several occasions the topological machinery can be made implicit, and the combinatorial question is settled directly by the lemmas of Sperner or Tucker.

2.1. Sperner and Tucker. A labeling of a simplicial complex $\mathrm{K}$ by a set $S$ is a map from the vertices of K to $S$; the label of a vertex is its image. Sperner's 
lemma gives a sufficient condition for the existence of a fully labeled facet, that is a facet whose vertices have pairwise distinct labels. (Sometimes the labels are called colors, and fully labeled faces are called colorful; we will avoid this terminology in this paper to avoid confusion with the colorful theorems in convex geometry that we discuss in Sections 3 and 5 )

Sperner's lemma. Assume that the vertices of a finite triangulation $\mathrm{T}$ of a simplex $\Delta$ are labeled so that any vertex lying in a face of $\Delta$ has the same label as one of the vertices of that face. If the vertices of $\Delta$ are given pairwise distinct labels, then the number of facets of $\mathrm{T}$ whose vertices have pairwise distinct labels is odd.

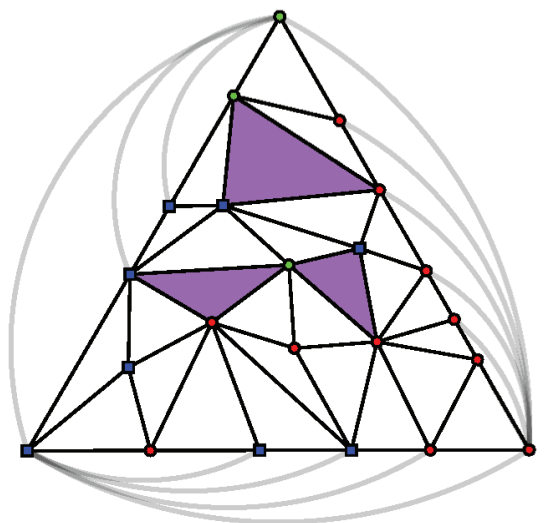

Figure 3. A Sperner labeling of a triangulation of $\Delta_{2}$ illustrated by colors. The fully labeled triangles are shown shaded. The gray edges augment the triangulation of $\Delta_{2}$ into a triangulation of $\mathbb{S}^{2}$.

We call a labeling that satisfies the assumptions of Sperner's lemma a Sperner labeling. A more general version holds for pseudomanifolds, i.e., for pure $d$-dimensional simplicial complexes where every face of dimension $d-1$ is contained in at most two facets. (Recall that a simplicial complex is pure if all its inclusion-maximal faces have the same dimension.) In particular, any triangulation of a $d$-dimensional manifold is a pseudomanifold of dimension $d$. The boundary of a pseudomanifold is the subcomplex generated by its $(d-1)$-dimensional simplices that are faces of exactly one $d$-dimensional simplex.

Proposition 2.1. Any labeling by $[d+1]$ of a d-dimensional pseudomanifold without boundary has an even number of fully labeled facets.

Proposition 2.1 follows from a simple parity argument. Consider the graph where the nodes are the facets and where the edges connect pairs of facets that share a $(d-1)$-dimensional face whose vertices use every label in $[d]$. This graph has only nodes of degree 0,1 , or 2 , so it consists of vertex-disjoint cycles and paths. The nodes of degree 1 are exactly the fully labeled facets, and there are evenly many of them (twice the number of paths). Coming back to the remark in introduction, this is where it is useful to understand why a house with an odd number of openings has a room with an odd number of openings.

Clearly, Sperner's lemma follows from Proposition 2.1. For this observe that any Sperner labeling of a $d$-dimensional simplex $\Delta_{d}$ extends into a triangulation 

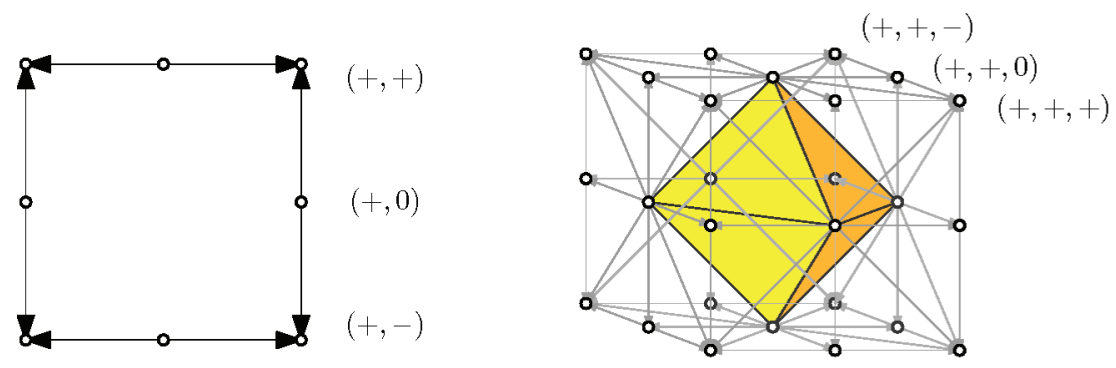

FIGURE 4. Geometric interpretation of the dominance graph for $\preceq$ in the octahedral Tucker lemma for $n=2$ (left) and $n=3$ (right).

of $\mathbb{S}^{d}$ where (i) the outer vertices of $\Delta_{d}$ form a fully labeled facet, and (ii) no other added facet is fully labeled (as illustrated in Figure 3). Knowing a vertex of degree 1 allows us easily, by path following, to find another one; in Section 2.4 we will present algorithmic applications of this idea. Other arguments can be used to prove Sperner's lemma [269].

Now we will state the octahedral Tucker lemma. This is a rather streamlined version of Tucker's lemma that already suffices for all our applications. Given vectors of signs $\boldsymbol{x}, \boldsymbol{y} \in\{+,-, 0\}^{n}$, we write $\boldsymbol{x} \preceq \boldsymbol{y}$ if every nonzero coordinate of $\boldsymbol{x}$ is the same as in $\boldsymbol{y}$. We let $\boldsymbol{x}^{+}$denote the set of indices $i$ such that $x_{i}=+$, and $\boldsymbol{x}^{-}$similarly. In particular, $\boldsymbol{x} \preceq \boldsymbol{y}$ if and only if $\boldsymbol{x}^{+} \subseteq \boldsymbol{y}^{+}$and $\boldsymbol{x}^{-} \subseteq \boldsymbol{y}^{-}$. Note that each vector of signs uniquely identifies a coordinate (sub)orthant, and that the order $\preceq$ indicates containment. There is an interpretation of $\left(\{+,-, 0\}^{n}, \preceq\right)$ as a simplicial complex illustrated in Figure 4. By $\pm a$ we mean a choice of one of the two scalars $-a$ or $a$.

Octahedral Tucker lemma. Let $\lambda:\{+,-, 0\}^{n} \backslash\{\mathbf{0}\} \rightarrow\{ \pm 1, \pm 2, \ldots, \pm m\}$ be such that $\lambda(-\boldsymbol{x})=-\lambda(\boldsymbol{x})$ for all $\boldsymbol{x}$. If $\lambda(\boldsymbol{x})+\lambda(\boldsymbol{y}) \neq 0$ for all $\boldsymbol{x} \preceq \boldsymbol{y}$, then $n \leq m$.

The octahedral Tucker lemma was apparently first stated explicitly by Ziegler [395, Lemma 4.1], following its implicit use by Matoušek [259] in his proof of the lower bound on the chromatic number of Kneser graphs from Tucker's lemma (see Section 5.11). Several classical proofs of Proposition 2.2 can be found in Matoušek's book [258]. As we explain in Section 2.2, the lemmas of Sperner and Tucker are indeed topological in that they essentially state that certain chain maps, namely those induced by the labeling maps, are nontrivial in simplicial homology with coefficients over $\mathbb{Z}_{2}$.

A more common version of Tucker's lemma deals with triangulations of a ball instead of an octahedron. Tucker's lemma gives a lower bound on the number of distinct labels used by labelings that avoid certain local patterns. We say that a triangulation $\mathrm{T}$ of $\mathbb{B}^{d}$ induces a symmetric triangulation of $\mathbb{S}^{d-1}$ if its boundary $\partial \mathrm{T}$ forms a centrally symmetric triangulation of $\mathbb{S}^{d-1}$. A labeling of a symmetric triangulation of $\mathbb{S}^{d}$ by integers is antipodal if antipodal vertices have opposite labels.

Proposition 2.2 (Tucker's lemma). Let $\mathrm{T}$ be a triangulation of $\mathbb{B}^{d}$ that induces a symmetric triangulation of $\mathbb{S}^{d-1}$. Let $\lambda$ be a labeling of the vertices of $\mathrm{T}$ by 

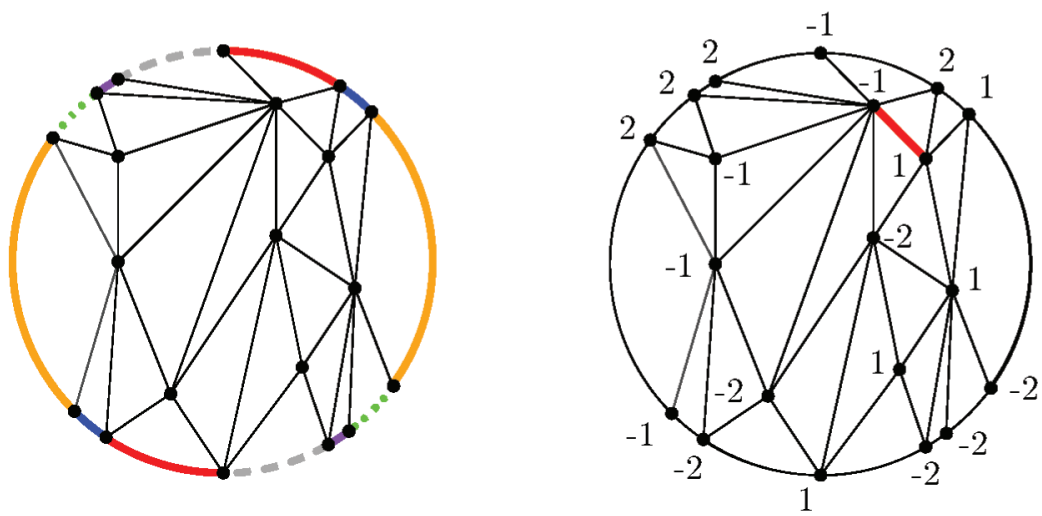

Figure 5. An illustration of Tucker's lemma: a triangulation of $\mathbb{B}^{2}$ that induces a symmetric triangulation of $\mathbb{S}^{1}$. On the left the antipodal simplices on the boundary are painted with the same color. On the right side, a labeling of the vertices that is antipodal on the boundary.

$\{ \pm 1, \ldots, \pm d\}$. If $\lambda(-\boldsymbol{v})=-\lambda(\boldsymbol{v})$ for every vertex $\boldsymbol{v}$ of $\partial \mathrm{T}$, then there exists an edge $\boldsymbol{u v}$ in $\mathrm{T}$ with $\lambda(\boldsymbol{u})+\lambda(\boldsymbol{v})=0$; see Figure 5 .

The octahedral version is obtained by applying Proposition 2.2 to the barycentric subdivision $\mathrm{T}$ of the $n$-dimensional cross-polytope $\diamond^{n}=\operatorname{conv}\left\{ \pm \boldsymbol{e}_{i}\right\}_{1 \leq i \leq n}$ [258, Theorem 2.3.2]. Indeed, consider a map $\lambda:\{+,-, 0\}^{n} \backslash\{\boldsymbol{0}\} \rightarrow\{ \pm 1, \ldots, \pm m\}$ such that $\lambda(-\boldsymbol{x})=-\lambda(\boldsymbol{x})$ for all $\boldsymbol{x}$ and $\lambda(\boldsymbol{x})+\lambda(\boldsymbol{y}) \neq 0$ for all $\boldsymbol{x} \preceq \boldsymbol{y}$. Any $\boldsymbol{x} \in$ $\{+,-, 0,\}^{n} \backslash\{\mathbf{0}\}$ can be interpreted as the vertex in $\mathrm{T}$ corresponding to the face $\operatorname{conv}\left(\left\{\boldsymbol{e}_{i}: i \in \boldsymbol{x}^{+}\right\} \cup\left\{-\boldsymbol{e}_{i}: i \in \boldsymbol{x}^{-}\right\}\right)$of $\nabla^{n}$. The edges in T connect pairs $\boldsymbol{x}$ and $\boldsymbol{y}$ such that $\boldsymbol{x} \preceq \boldsymbol{y}$. Defining $\lambda(\mathbf{0})=m+1$, we get a labeling of all vertices of $\mathrm{T}$ satisfying $\lambda(-\boldsymbol{v})=-\lambda(\boldsymbol{v})$ for every vertex $\boldsymbol{v}$ of $\partial \mathrm{T}$ and having no edge $\boldsymbol{u} \boldsymbol{v}$ in $\mathrm{T}$ with $\lambda(\boldsymbol{u})+\lambda(\boldsymbol{v})=0$. By Proposition 2.2, we must have $m+1>n$.

As stated in the introduction, Tucker's lemma is equivalent to the fact that for any triangulation of the projective plane and any 2-coloring of its vertices, one of the color classes spans a noncontractible cycle. Indeed, such a 2-coloring can be seen as a 2-coloring of a triangulation of the disk, with symmetric vertices of the boundary getting identical colors. If all monochromatic cycles were contractible, we could easily choose a sign for each vertex and get a labeling that would contradict Tucker's lemma. The reverse implication is also easy and is left to the reader.

Consider a triangulation $\mathrm{T}$ as in Proposition 2.2 and a labeling $\lambda$ of its vertices by $\{ \pm 1, \ldots, \pm m\}$; a $k$-dimensional face of $\mathrm{T}$ is alternating if its vertices can be indexed $\boldsymbol{v}_{i_{0}}, \ldots, \boldsymbol{v}_{i_{k}}$ so that $0<\lambda\left(\boldsymbol{v}_{i_{0}}\right)<-\lambda\left(\boldsymbol{v}_{i_{1}}\right)<\cdots<(-1)^{k} \lambda\left(\boldsymbol{v}_{i_{k}}\right)$ or if $0>\lambda\left(\boldsymbol{v}_{i_{0}}\right)>-\lambda\left(\boldsymbol{v}_{i_{1}}\right)>\cdots>(-1)^{k} \lambda\left(\boldsymbol{v}_{i_{k}}\right)$. In the first case we call the simplex positively alternating; in the second case, negatively alternating. A lemma due to Fan [160] generalizes Tucker's lemma in terms of a parity counting of alternating simplices.

Theorem 2.3 (Fan's lemma). Let $\mathrm{T}$ be a triangulation of $\mathbb{B}^{d}$ that induces a symmetric triangulation of $\mathbb{S}^{d-1}$. Let $\lambda$ be a labeling of the vertices of $\mathrm{T}$ by $\{ \pm 1, \ldots, \pm m\}$ such that $\lambda(-\boldsymbol{v})=-\lambda(\boldsymbol{v})$ for every vertex $\boldsymbol{v}$ of $\partial \mathrm{T}$. If no two 
adjacent vertices have opposite labels, then $\mathrm{T}$ has an odd number of alternating facets.

Fan's lemma readily implies Tucker's lemma since the existence of an alternating $d$-dimensional face implies $m \geq d+1$. Going in the other direction, it was only recently observed by Alishahi [15] that an existential version of Fan's lemma is easily derived from Tucker's lemma.

Let us illustrate that, surprisingly, Fan's lemma can be easier to prove than Tucker's lemma: this is one example where a stronger hypothesis facilitates induction. We give an inductive proof for a flag of hemispheres, i.e., a triangulation $\mathrm{T}$ of $\mathbb{B}^{d}$ such that the restriction of $\mathrm{T}$ on $H_{i}^{+}$and on $H_{i}^{-}$triangulates each of them, where $H_{i}^{+}$and $H_{i}^{-}$are the $i$-dimensional hemispheres

$$
\begin{aligned}
& H_{i}^{+}=\left\{\boldsymbol{x} \in \mathbb{S}^{d-1}: x_{i+1} \geq 0, x_{i+2}=\cdots=x_{d}=0\right\}, \\
& H_{i}^{-}=\left\{\boldsymbol{x} \in \mathbb{S}^{d-1}: x_{i+1} \leq 0, x_{i+2}=\cdots=x_{d}=0\right\} .
\end{aligned}
$$

(Prescott and $\mathrm{Su}$ 318] gave another combinatorial constructive proof for this special case.) Consider the graph whose nodes are the facets of $\mathrm{T}$ and whose edges connect pairs of facets that share a $(d-1)$-dimensional face that is positively alternating. We augment this graph with an extra node $s$ and add edges connecting $s$ to all facets of $\mathrm{T}$ that have a $(d-1)$-dimensional positively alternating face on $\partial \mathrm{T}$; in a sense, $s$ represents the outer facet. Apart from $s$, all nodes have degree 0,1 , or 2. The nodes of degree 1 are exactly the $d$-dimensional alternating facets. The triangulation $\mathrm{T}$ refines $H_{d-1}^{+}$, which is homeomorphic to $\mathbb{B}^{d-1}$. So by induction in dimension $d-1$, the number of $(d-1)$-dimensional alternating faces of $\partial \mathrm{T}$ in $H_{d-1}^{+}$is odd; the same holds for the $(d-1)$-dimensional faces of $\partial \mathrm{T}$ in $H_{d-1}^{-}$. The symmetry of $\partial \mathrm{T}$ and that of the labeling imply that the degree of $s$ is odd; it follows that there is an odd number of $d$-dimensional alternating facets in $\mathrm{T}$.

The above elementary proof requires that the triangulation restricts nicely to lower-dimensional spheres to allow induction. Two proofs of Theorem 2.3 can be found in the literature, both for an equivalent version with a sphere instead of a ball (later we will explain this equivalence for our own proof of Theorem 2.3). On the one hand, Živaljević [397] observed that the labeling is essentially a classifying map that is unique up to $\mathbb{Z}_{2}$-homotopy, so the number of alternating facets $(\bmod 2)$ reformulates as the cap product of a certain cohomology class with a certain homology class. On the other hand, Musin 289 builds a simplicial $\mathbb{Z}_{2}$-map from the triangulation to a $d$-dimensional polytope for which the following holds: a simplex is alternating if and only if its image by this simplicial map contains 0 in its convex hull; a degree argument then allows us to conclude. It turns out that Alishahi's idea to derive Fan's lemma from Tucker's lemma leads to a short proof of Theorem 2.3. also based on a degree argument. Before we spell out this (original) proof, let us stress that the following question remains.

Open Problem 2.4. Give a direct combinatorial proof of Fan's lemma (as stated in Theorem 2.3) and of Tucker's lemma (Proposition 2.2) valid for any centrally symmetric triangulation.

Let us now prove Theorem 2.3 for an arbitrary triangulation $\mathrm{T}$ of $\mathbb{B}^{d}$. Let $\lambda$ be a labeling of the vertices of $T$ satisfying the conditions of Theorem 2.3 . We first turn $\mathrm{T}$ into a triangulation $\mathrm{T}^{\prime}$ of $\mathbb{S}^{d}$ by gluing two antipodal copies of $\mathbb{B}^{d}$, each triangulated by $\mathrm{T}$; notice that the number of positively alternating facets 
of $\mathrm{T}^{\prime}$ equals the number of alternating facets of $\mathrm{T}$ (both positive and negative ones), since the negatively alternating facets in one copy of $T$ become positively alternating in the other copy. We define next a labeling $\mu$ of the vertices of $\mathrm{sd}^{\mathrm{T}^{\prime}}$ by $\{ \pm 1, \ldots, \pm(d+1)\}$ : a vertex $v$ of $\operatorname{sd}^{\mathrm{T}^{\prime}}$ corresponds to a simplex $\tau_{v}$ of $\mathrm{T}^{\prime}$, and we set the absolute value of $\mu(v)$ to be the number of vertices of the largest alternating face of $\tau_{v}$, and its sign according to alternation. This sign is defined uniquely since there cannot be maximal alternating faces of both types in $\tau$ (this can be checked using the fact that no adjacent vertices in $T$ can have opposite labels). Now, a crucial observation is that if $\sigma$ is an alternating facet (for $\lambda$ ), then $\operatorname{sd} \sigma$ contains exactly one alternating facet (for $\mu$ ) of the same type; and if $\sigma$ is not an alternating facet, then $\operatorname{sd} \sigma$ contains no alternating facet. At this point, to establish Theorem 2.3 , it suffices thus to prove that sd $\mathrm{T}^{\prime}$ contains an odd number of positively alternating facets. This fact follows from basic degree theory. The assumptions on $\lambda$ guarantee that $\mu$ induces an antipodal simplicial map from sd $\mathrm{T}^{\prime}$ to $\partial \diamond^{d+1}$, the boundary of the $(d+1)$-dimensional cross-polytope, whose vertices are identified with the elements in $\{ \pm 1, \ldots, \pm(d+1)\}$. Any antipodal self-map of $\mathbb{S}^{d}$ is of odd degree [142, Theorem 4.3.32]. Thus, denoting by $t \in C_{d}\left(\operatorname{sd~} \mathrm{T}^{\prime}, \mathbb{Z}_{2}\right)$ the formal sum of all facets of $\mathrm{sd}^{\prime}$ and by $z \in C_{d}\left(\partial \diamond^{d+1}, \mathbb{Z}_{2}\right)$ the formal sum of all facets of $\partial \diamond^{d+1}$, we must have $\mu_{\sharp}(t)=z$. The only simplices that are mapped to the simplex $\left\{+1,-2, \ldots,(-1)^{d}(d+1)\right\}$ by $\mu_{\sharp}$ are the positively alternating ones, so there are an odd number of them.

2.2. Continuous versions. One of the most famous theorems about fixed points is due to the Dutch mathematician L. E. J. Brouwer and states that any continuous function from a ball into itself has a fixed point. Brouwer's theorem is often seen as a continuous version of Sperner's lemma (without the oddness assertion): they can be deduced easily from one another.

Let us sketch how Brouwer's theorem follows from Sperner's lemma (we discuss the other direction in Section 2.3). Contrary to Brouwer's original proof, which says nothing about how to find the fixed point or a good approximation of it, this proof has computational implications (see Section 2.4). Without loss of generality we take the $d$-dimensional standard simplex $\Delta_{d} \subset \mathbb{R}^{d+1}$ as a realization of a ball (it is easy to set up a homeomorphism). Then we triangulate the simplex $\Delta_{d}$ and design a labeling of that triangulation tailored to the continuous function $f$ under consideration. Specifically, we associate to a vertex $\boldsymbol{v}$ of the triangulation the label $i$ if the $i$ th barycentric coordinate of $\boldsymbol{v}$ is larger than the $i$ th barycentric coordinate of its image $f(\boldsymbol{v})$. (So, intuitively, if $\boldsymbol{v}$ is labeled $i$, then $f$ moves $\boldsymbol{v}$ away from the $i$ th vertex of the standard simplex.) Note that, unless the vertex $\boldsymbol{v}$ is a fixed point, there must be at least one such index. If there are several such indices, simply make an arbitrary choice among them. Now, the labeling we provided satisfies the assumptions of Sperner's lemma, so we can find a fully labeled simplex of T such that the $i$ th barycentric coordinate of the vertex labeled $i$ is decreased by $f$. Retriangulate $\Delta_{d}$ again and again adding more and more points in such a way that the maximum diameter of the simplices appearing in the triangulation goes to zero in the limit. At each step we find a fully labeled simplex. The barycenters of all such simplices will produce an infinite sequence of points and, since it is a bounded sequence, it contains a convergent subsequence. Let $\boldsymbol{x}^{*}$ be the limit of this subsequence. Since the map $f$ is continuous, the $i$ th barycentric coordinate of $\boldsymbol{x}^{*}$ is larger than or equal to the $i$ th barycentric coordinate of $f\left(\boldsymbol{x}^{*}\right)$ for every $i$ and therefore $\boldsymbol{x}^{*}$ is a fixed point of the map. 


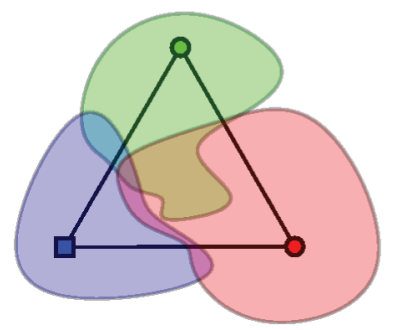

Figure 6. An illustration of the KKM lemma in two dimensions.

The Knaster-Kuratowski-Mazurkiewicz theorem, also known as the KKM theorem, is a classical consequence of Sperner's lemma or Brouwer's theorem. It is used, for instance, in game theory or for the study of variational inequalities. Consider the $d$-dimensional simplex $\Delta_{d}=\operatorname{conv}\left\{\boldsymbol{e}_{i}: 1 \leq i \leq d+1\right\}$ and $d+1$ closed sets $C_{1}, C_{2}, \ldots, C_{d+1}$ in $\mathbb{R}^{d}$. This theorem (illustrated in Figure 6 ) ensures that if for every $I \subseteq[d+1]$ the face $\operatorname{conv}\left\{\boldsymbol{e}_{i}: i \in I\right\}$ of $\Delta_{d}$ is covered by $\bigcup_{i \in I} C_{i}$, then $\bigcap_{i=1}^{d+1} C_{i}$ is nonempty. This statement is somehow reminiscent of Helly's theorem. A corollary of the KKM theorem can actually be used to prove it; see Section 3.2 . This corollary states that if $d+1$ closed sets $C_{1}, C_{2}, \ldots, C_{d+1}$ are such that each of them contains a distinct facet of $\Delta_{d}$ and such that their union covers $\Delta_{d}$, then their intersection is nonempty (this statement is also called a dual KKM theorem in [32]). To see that it is a consequence of the KKM theorem, assign number $i$ to the facet covered by $C_{i}$; number the vertices of $\Delta_{d}$ so that vertex $i$ is on facet $i$; the $C_{i}$ 's satisfy then the condition of the KKM theorem.

Several variations of the original KKM theorem exist. Gale 177 proved the following colorful version: given $d+1$ different KKM covers $\left\{C_{i}^{1}\right\}_{i=1}^{d+1},\left\{C_{i}^{2}\right\}_{i=1}^{d+1}, \ldots$, $\left\{C_{i}^{d+1}\right\}_{i=1}^{d+1}$ of the $d$-simplex, there exists a permutation $\pi$ of the numbers $[d+1]$ such that $\bigcap_{i=1}^{d+1} C_{\pi(i)}^{i} \neq \varnothing$. Clearly, choosing all the covers to be the same recovers the classical version of KKM stated above. Gale's colorful KKM theorem has an intuitive interpretation first stated by Gale himself: "if each of three people paint a triangle red, white, and blue according to the KKM rules of covering, then there will be a point which is in the red set of one person, the white set of another, the blue of the third". A recent strengthening of this colorful theorem 32 states that, given $d$ KKM covers $\left\{C_{i}^{1}\right\}_{i=1}^{d+1},\left\{C_{i}^{2}\right\}_{i=1}^{d+1}, \ldots,\left\{C_{i}^{d}\right\}_{i=1}^{d+1}$ of the $d$-simplex $\Delta_{d}$, then there exist a point $\boldsymbol{x}$ in $\Delta_{d}$ and $d+1$ bijections $\pi_{i}:[d] \rightarrow[d+1] \backslash\{i\}$ for $i=1, \ldots, d+1$, such that $\boldsymbol{x} \in \bigcap_{j=1}^{d} C_{\pi_{i}(j)}^{j}$ for every $i$. It is interesting to note the proofs of these colorful results combine degree theory with Birkhoff's theorem on doubly stochastic matrices. Finally, we note that 291 has common generalizations of Sperner, Tucker, KKM, and Fan.

Another fascinating and very useful consequence of Brouwer's theorem is Kakutani's 1941 fixed-point theorem. It deals, not with real-valued functions, but with set-valued functions, where points are mapped to subsets. For a suitable notion of continuity, it ensures that for any continuous function $F$ mapping points of a ball to convex subsets of it there is an $\boldsymbol{x}$ such that $\boldsymbol{x} \in F(\boldsymbol{x})$. Kakutani's theorem is especially useful in game theory, its most traditional application being the Nash theorem; see Section 4 
Similar to Sperner's lemma, Tucker's lemma has continuous and covering versions. The continuous version is the celebrated Borsuk-Ulam theorem, which has many applications in discrete geometry, combinatorics, and topology. It asserts that there is no continuous function from $\mathbb{S}^{d}$ into $\mathbb{S}^{d-1}$ that commutes with the central symmetry. Nice proofs of the Borsuk-Ulam theorem from Tucker's lemma, as well as equivalent formulations and many applications, can be found for instance in the books of Matoušek [258, Chapter 2.3] and de Longueville [133, Chapter 1]. Just as KKM is the covering version of Sperner's lemma, Tucker's lemma has a covering version, the Lysternik-Schnirel'mann theorem [250]. It states that if the sphere $\mathbb{S}^{d}$ is covered by $d+1$ closed subsets, then one of the sets must contain two antipodal points. This theorem and some of its extensions (e.g., those due to Fan) have found many applications in other areas of mathematics, for instance, for the KKM theorem, in the study of variational inequalities.

2.3. Generalizations and variations. A labeling $\lambda$ of a simplicial complex $\mathrm{T}$ by a set $S$ can be interpreted as a map from the vertices of T to the vertices of some abstract simplicial complex $\mathrm{K}$ with vertex set $S$. This viewpoint leads to several interesting developments.

A first idea is to extend $\lambda$ into a linear map $f$ from $|\mathrm{T}|$ into $|\mathrm{K}|$. For a Sperner labeling, $f$ maps $\Delta_{d}$ into itself. Composing $f$ with a suitable orthogonal transformation ensures that any fixed point of the resulting map, which must exist by Brouwer's theorem, lies in a fully labeled simplex; this is the standard proof of Sperner's lemma from Brouwer's theorem [133, Section 1.1]. Using this idea, Sperner's lemma was generalized to triangulations of arbitrary polytopes by De Loera, Peterson, and $\mathrm{Su}$ [131.

Proposition 2.5 (Polytopal Sperner lemma). Let $P \subset \mathbb{R}^{d}$ be a polytope with $n$ vertices, let $\mathrm{T}$ be a triangulation of $P$, and let $\lambda$ be a labeling of the vertices of $\mathrm{T}$ by $[n]$. If the vertices of $P$ have pairwise distinct labels and every vertex of $\mathrm{T}$ lying on a face $F$ of the boundary of $P$ has same label as some vertex of $F$, then $\mathrm{T}$ contains at least $n-d$ fully labeled facets.

The gist of the proof is that $\lambda$ extends into a piecewise linear map from $|\mathrm{T}|$ to $P$ (as illustrated in Figure 7). This map can be shown to be surjective, so its image provides a covering of $P$ by simplices spanned by its vertices. The number of such simplices required to cover $P$ is at least $n-d$, and each of them is the image (under the extension of $\lambda$ ) of a fully labeled facet of $T$. This approach generalizes to nonconvex polyhedral manifolds [273] and broader classes of manifolds [289,290]. The reader interested in recent progress on lower bounds for the number of fully labeled facets is refered to the work of Asada et al. 32.

Another idea is to extend $\lambda$ into a chain map $\lambda_{\sharp}: C_{\bullet}(\mathrm{T}, R) \rightarrow C_{\bullet}(\mathrm{K}, R)$. Depending on the coefficients ring $R$ and the complex $\mathrm{K}$, one gets different generalizations of classical statements. This extension goes as follows. Send every simplex $\left\{\boldsymbol{v}_{0}, \ldots, \boldsymbol{v}_{k}\right\}$ of $\mathrm{T}$ to $\left\{\lambda\left(\boldsymbol{v}_{0}\right), \ldots, \lambda\left(\boldsymbol{v}_{k}\right)\right\}$ if the $\lambda\left(\boldsymbol{v}_{i}\right)$ are pairwise distinct and to 0 otherwise; the linear extension of this map to $C \bullet\left(T, \mathbb{Z}_{2}\right)$ is the map $\lambda_{\sharp}$, and it commutes with $\partial$ (it is a chain map).

For $R=\mathbb{Z}_{2}$, we obtain a short proof by induction of Sperner's lemma. Assume that $\lambda$ is a Sperner labeling of a triangulation $\mathrm{T}$ of $\Delta_{d}$, so $\lambda_{\sharp}: C_{\bullet}\left(\mathrm{T}, \mathbb{Z}_{2}\right) \rightarrow$ $C \bullet\left(\sigma_{d}, \mathbb{Z}_{2}\right)$. Let $t$ denote the sum of $d$-simplices of $\mathrm{T}$, and let $\sigma$ denote the unique 

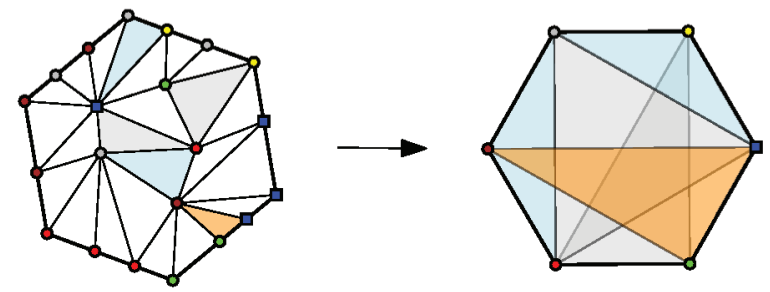

FIgURE 7. A Sperner-type labeling of a triangulation of a polygon and the associated surjective map to the polygon.

$d$-simplex of $\sigma_{d}$. Observe that $\lambda_{\sharp}(t)=\ell \sigma$, where $\ell$ is the number of fully labeled simplices in $\mathrm{T} \bmod 2$. A simple induction shows that $\lambda_{\sharp}(\partial t)=\partial \sigma$. Thus, $\partial \lambda_{\sharp}(t)=\lambda_{\sharp}(\partial t) \neq 0$. It comes that $\ell \neq 0$, and $\mathrm{T}$ has an odd number of fully labeled simplices.

For $R=\mathbb{Z}$, the same proof gives a Sperner-type result for oriented simplices. The orientation of a fully labeled simplex $\left\{\boldsymbol{v}_{1}, \boldsymbol{v}_{2}, \ldots, \boldsymbol{v}_{d+1}\right\}$ of $\mathrm{T}$, where $\boldsymbol{v}_{i}$ has label $i$, is defined as the sign ( 1 or -1$)$ of the determinant

$$
\left|\begin{array}{cccc}
\boldsymbol{v}_{1} & \boldsymbol{v}_{2} & \ldots & \boldsymbol{v}_{d+1} \\
1 & 1 & \ldots & 1
\end{array}\right| .
$$

Specifically, this proves that in any Sperner labeling of a triangulation of $\Delta_{d}$ by $[d+1]$ the orientations of the fully labeled simplices add up to 1 [161, Theorem 2]. This approach also yields a proof of (a signed version of) Fan's lemma [133, Theorem 1.10], again for triangulations with flags of hemispheres.

For $R=\mathbb{Z}_{q}$, this idea leads to generalizations of the lemmas of Tucker and Fan with more than two signs [201,272]. The general insight, following a generalization by Dold [144] of the Borsuk-Ulam theorem, is to replace $\mathbb{S}^{d}$ and the antipodality by a suitable simplicial complex on which $\mathbb{Z}_{q}$ acts freely. The resulting $\mathbb{Z}_{q}$-Fan lemmas actually provide combinatorial proofs of Dold's theorem. The deduction of the $\mathbb{Z}_{q}$-Fan lemmas from their $\mathbb{Z}_{q}$-Tucker versions works as explained above in the $\mathbb{Z}_{2}$ case 15 .

A variant of the above argument yields a new and elementary proof of a relative of Sperner's lemma, due to Meshulam [270. It is a powerful result that has found many applications in graph theory and in discrete geometry, such as the recently found generalization of the colorful Carathéodory theorem by Holmsen and Karasev [212]. We come back later to some of its applications in discrete geometry (Section 3.1) and in graph theory (Section 5.2). Meshulam's lemma was first explicitly derived from (a special version of) Leray's acyclic cover theorem (its first use was implicit; see Kalai and Meshulam [222, Proposition 3.1] for an explicit statement and proof for rational homology).

For the sake of presentation, we consider here homology over $\mathbb{Z}_{2}$ but the proof generalizes, mutatis mutandis, to an arbitrary ring. Given a simplicial complex $\mathrm{K}$ and a subset $X$ of its vertices, we denote by $\mathrm{K}[X]$ the simplicial complex formed by the simplices of $\mathrm{K}$ whose vertices are in $X$.

Proposition 2.6 (Meshulam's lemma). Let $\lambda$ be a labeling of the vertices of a simplicial complex $\mathrm{K}$ by $[d+1]$. If $\widetilde{H}_{|I|-2}\left(\mathrm{~K}\left[\lambda^{-1}(I)\right]\right)$ is trivial for every nonempty $I \subseteq[d+1]$, then $\mathrm{K}$ contains a fully labeled d-dimensional face. 
The proof goes as follows. Let $\lambda_{\sharp}: C_{\bullet}(\mathrm{K}) \rightarrow C_{\bullet}\left(\sigma_{d}\right)$ denote the chain map induced by $\lambda$ and recall that it maps simplices with repeated labels to 0 . We build a chain map $f_{\sharp}: C_{\bullet}\left(\sigma_{d}\right) \rightarrow C_{\bullet}(\mathrm{K})$ such that $\lambda_{\sharp} \circ f_{\sharp}=\mathrm{id}_{\sigma_{d}}$; the identity $\left(\lambda_{\sharp} \circ f_{\sharp}\right)([d+1])=$ $[d+1]$ then ensures that $f_{\sharp}([d+1])$ contains an odd number of fully labeled simplices, proving the statement. We build $f_{\sharp}$ by increasing dimension. We start by setting $f_{\sharp}(\{i\})$ to be some (arbitrary) vertex in $\lambda^{-1}(\{i\})$ for every $i \in[d+1]$; this is possible because $\widetilde{H}_{-1}\left(\mathrm{~K}\left[\lambda^{-1}(\{i\})\right]\right)$ is trivial. Assume that $f_{\sharp}$ is defined over all chains up to dimension $k$, that it maps any subset $I$ of cardinality at most $k+1$ to $C \cdot\left(\mathrm{K}\left[\lambda^{-1}(I)\right]\right)$, and that it commutes with the boundary operator. Now, for any subset $I$ of cardinality $k+2$, we have

$$
\partial\left(\sum_{i \in I} f_{\sharp}(I \backslash\{i\})\right)=\partial f_{\sharp}\left(\sum_{i \in I} I \backslash\{i\}\right)=\partial f_{\sharp}(\partial I)=f_{\sharp}\left(\partial^{2} I\right)=0,
$$

so the $k$-chain $\sum_{i \in I} f_{\sharp}(I \backslash\{i\})$ is a cycle in $C_{\bullet}\left(\mathrm{K}\left[\lambda^{-1}(I)\right]\right)$. The assumption of the lemma ensures that it is the boundary of some chain $\gamma \in C_{|I|-1}\left(\mathrm{~K}\left[\lambda^{-1}(I)\right]\right)$, and we set $f_{\sharp}(I)$ to be $\gamma$. To see that $\left(\lambda_{\sharp} \circ f_{\sharp}\right)(I)=I$ for any $I \subseteq[d+1]$, first note that this is straightforward for $|I|=1$. For the general case, remark that

$$
\partial \lambda_{\sharp}\left(f_{\sharp}(I)\right)=\lambda_{\sharp}\left(\partial f_{\sharp}(I)\right)=\sum_{i \in I} \lambda_{\sharp}\left(f_{\sharp}(I \backslash\{i\})\right)=\sum_{i \in I} I \backslash\{i\},
$$

so we can assume by induction that $\partial \lambda_{\sharp}\left(f_{\sharp}(I)\right)=\partial I$. We conclude by observing that $f_{\sharp}(I) \in C_{|I|-1}\left(\mathrm{~K}\left[\lambda^{-1}(I)\right]\right)$ means that $\lambda_{\sharp}\left(f_{\sharp}(I)\right)$ is supported only on subsets of $I$, so it must be that $\lambda_{\sharp}\left(f_{\sharp}(I)\right)=I$.

The parity argument used to prove Proposition 2.1 can also be found, specialized to a certain labeled pseudomanifold, in Scarf's proofs of the integer Helly theorem 340, and his classical lemma in game theory 339. There is a related unbounded polar version that will be useful in Section 5.3 .

Corollary 2.7 ([232, Theorem 3]). Let $P$ be a d-dimensional pointed polyhedron whose characteristic cone is generated by dinearly independent vectors and whose facets are labeled by $[d]$. If no facet containing the ith extreme direction is labeled $i$, then there exists a extreme point incident to a facet of each label.

Another recent variation of Sperner's lemma, motivated by applications in approximation algorithms, asks for the minimum possible number of facets in the Sperner labeling that must be nonuniquely labeled. Mirzakhani and Vondrak [281, 282 settled this question for certain triangulations of the simplex, for which they provided the optimal Sperner labeling. They then proposed two very interesting open questions.

Open Problem 2.8. Is there a theorem that interpolates between the result above (lower bound on the number of simplices with at least two different colors) and the original Sperner's lemma (lower bound on the number of simplices with vertices of different color) by predicting how many simplices are colored with at least $j$ different colors? How does such a theorem depend on the structure of the particular triangulation?

It must be mentioned that Tucker-type theorems and Sperner-type theorems are related to each other in interesting ways. For example, it is known that the BorsukUlam theorem implies the Brouwer fixed-point theorem, but at the combinatorial 
level Nyman and Su [306] proved that Fan's lemma implies Sperner's theorem too. Other interconnections can be found in 318, 359.

2.4. Computational considerations. The proof of Sperner's lemma given for Proposition 2.1 builds a graph where every vertex has degree 0,1 , or 2 , then exhibits a vertex of degree 1 and argues that any other vertex of degree 1 must correspond to a fully labeled simplex. This provides a simple algorithm for finding a fully labeled simplex: just follow the path! We can combine this simple path-following algorithm for finding fully labeled simplices with the proof of Brouwer's theorem, presented at the beginning of Section 2.2 and provide a method for finding an approximate fixed point of the continuous map $f$. Again assume we are given a continuous map $f: \Delta_{d} \rightarrow \Delta_{d}$ and an $\varepsilon>0$. Our goal is to find $\boldsymbol{x} \in \Delta_{d}$ such that $\|f(\boldsymbol{x})-\boldsymbol{x}\| \leq \varepsilon$. For this, it suffices to compute a triangulation of $\Delta_{d}$ with simplices of diameter sufficiently small, depending on $\varepsilon$ and the modulus of continuity of $f$, label it as in the proof of Brouwer's theorem, and any vertex $\boldsymbol{x}$ of a fully labeled simplex does the job (this fact is more easily formalized by using the $\ell_{\infty}$ norm on the barycentric coordinates). This template of proof was first presented in 338 and is quite flexible, e.g., it applies to noncontracting functions. We left out many details, for instance the choice of the triangulation to speed up the algorithm and the estimation of the modulus of continuity. The interested reader can find more details on methods to compute approximate fixed points based on these ideas in [374].

The theory of computational complexity is a formal way for computer scientists to classify the inherent difficulty of computational problems. Families of problems, called complexity classes, collect problems of equivalent difficulty (a complete introduction can be found in 30 ). Famous complexity classes of course include the class $\mathrm{P}$ and the class NP, but here and in Section 3.4 we briefly discuss the complexity classes that relate to computational versions of our five central theorems.

The path-following algorithm for computing the fully labeled simplex for Sperner's lemma is representative of the PPAD class, a complexity class well-suited for studying computational problems whose solution is known to exist, but finding it is not that easy. This class was presented by Papadimitriou [314; see also 304. The prototypical problem of the class PPAD (which abbreviates Polynomial Parity Argument for Directed graphs) assumes an underlying directed graph where every vertex has in- and out-degrees at most 1; the graph may be implicit, and all that is required is the existence of a function that computes the neighborhood of a given vertex in time polynomial in the encoding of that vertex. The PPAD problem asks, given the encoding of an unbalanced vertex (that is, with different in- and outdegrees), to compute the encoding of another unbalanced vertex. (Note that this computational problem does not easily reduce to a meaningful associated decision problem, since the existence of this other unbalanced vertex follows systematically from parity considerations.) A problem is in the PPAD class if it has a polynomial reduction to the PPAD problem, and a problem from the PPAD class is PPADcomplete if every problem from the PPAD class has a polynomial reduction to that problem. (All reductions here are meant in the usual sense of polynomial reductions; see [304, §2].) PPAD-completeness results imply conditional lower bounds in the following sense: one cannot solve a PPAD-complete problem substantially faster than by path following, unless there is also a substantially better method than path following for the PPAD problem (and similarly for every other problem in the PPAD class). As in the case of the $\mathrm{P}$ vs NP problem, failure over time to improve 
on even the most streamlined of these problems supports the conjecture that none of these methods can be substantially improved.

The Sperner problem - where one asks, given a Sperner labeling, for a fully labeled simplex - is well-known to be PPAD-complete in any fixed dimension. (Formalizing this problem properly requires some care, for instance the definition of a canonical sequence of triangulations with simplices of vanishing diameter, which we do not discuss.) Papadimitriou's seminal paper, which started the theory of PPAD problems 314, settled the three-dimensional case and listed the two-dimensional case as an important open problem; it was settled in the positive a decade later by Chen and Deng [103.

While Tucker's lemma can also be proved via a path-following argument [167, the computational problem associated to Tucker's lemma is not known to belong to the PPAD class: contrary to Sperner's lemma, there is no natural orientation of the edges of the underlying graph. The suitable complexity class to use for the Tucker problem is a superclass of the PPAD class, the class PPA. Here PPA abbreviates Polynomial Parity Argument for graphs. This class was introduced at the same time as the PPAD class, its definition is almost the same: instead of working with directed graphs, one works with undirected ones. The underlying graph defining the PPA problem has all its vertices of degree at most 2 and asks, given the encoding of a vertex of degree 1 , to output the encoding of another degree 1 vertex. PPA contains PPAD, but it is a famous problem to decide whether the two classes are actually the same; this was already asked in the paper founding this topic [314]. Experts believe that these two classes are different [191].

Open Problem 2.9. Are the complexity classes PPA and PPAD equal?

As for the Sperner problem, the Tucker problem is PPA-complete already in dimension 2 (see Aisenberg et al. [11, who corrected an earlier, wrong, assertion of PPAD-ness). Pálvölgyi [312 introduced a clean variation of this problem-the octahedral Tucker problem - together with the open question below:

Given a function $\lambda:\{+,-, 0\}^{n} \backslash\{\mathbf{0}\} \rightarrow\{ \pm 1, \pm 2, \ldots, \pm m\}$, computable in time polynomial in $n$ and such that $n>m$ and $\lambda(-\boldsymbol{x})=$ $-\lambda(\boldsymbol{x})$ for all $\boldsymbol{x}$, compute $\boldsymbol{x} \preceq \boldsymbol{y}$ such that $\lambda(\boldsymbol{x})+\lambda(\boldsymbol{y})=0$.

Note that contrary to the Tucker problem we just discussed, the dimension is not part of the input. The computational complexity of the algorithmic version of the octahedral Tucker lemma had been an outstanding challenging problem, but the paper [137] resolved this problem by proving octahedral Tucker lemma to be PPA-complete.

We will also discuss in our applications (particularly in section 4.1.2) some consequences of the lemmas of Sperner or Tucker whose computational versions may be complete for two related complexity classes. The first class is FIXP, introduced by Etessami and Yannakakis [158. It consists of the problems whose resolution on an instance $\ell$ reduces to the computation of a fixed point of some function $F_{\ell}$ that can be expressed by the operations $\{+, *,-, /$, max, $\min \}$ with rational constants and functions and computed in time polynomial in the size of $\ell$; this extends PPAD, which coincides with the case of linear functions. The second class, called $\exists \mathbb{R}$ [341] (sometimes abbreviated ETR for existence of real solutions, see [181]), studies problems that reduce to deciding the emptiness of a general semi-algebraic 
set, i.e., the set of real solutions of a system of inequalities with polynomials as constraints. These two complexity classes are relevant in Section 4.

\section{Combinatorial Convexity}

We now focus on three classical combinatorial theorems about convex sets first identified in the early 20th century. These are the theorems of Carathéodory, Helly, and Tverberg. The importance of convexity in applications, and hence of these three theorems, owes much to the computational effectiveness of convex optimization algorithms both in practice and in theory [58, 76]. This encourages applied mathematicians to look for convexity or for ways to approximate complicated sets using convex sets. Surprisingly, convexity appears in unexpected settings.

Extensive surveys were devoted to (subsets and variations on) some of these three theorems by Danzer, Grünbaum, and Klee [120, Eckhoff [152, Holmsen and Wenger 211] and Amenta, De Loera, and Soberón [28]. An account of early variations of Carathéodory's theorem is in the memoir by Reay 322 .

There is an abundant literature on axiomatic convexity, which studies analogues of the theorems of Carathéodory, Helly, and Tverberg, not over Euclidean spaces as we do here, but over purely combinatorial abstract settings, for instance in the convexity spaces defined by arbitrary graphs, finite geometries, matroids, greedoids, etc. The three theorems play a significant and interesting role there too, but we do not cover this topic here. We refer the interested reader to the references [148, 230, 380.

3.1. Carathéodory. We will first consider Carathéodory-type theorems that certify membership of a point in the convex hull of a set via linear nonnegative combinations. The original theorem of Carathéodory [89] asserts that any point in the convex hull of a finite point set in $\mathbb{R}^{d}$ is a convex combination of some at most $d+1$ of these points. Equivalently, if a vector $\boldsymbol{b}$ belongs to the cone of $X=\left\{\boldsymbol{v}_{1}, \boldsymbol{v}_{2}, \ldots, \boldsymbol{v}_{n}\right\} \subset \mathbb{R}^{d}$ (i.e., the positive hull of all nonnegative real linear combinations of vectors in $X$ ), then $\boldsymbol{b}$ is a positive combination of at most $d$ vectors of $X$. To see this, let $A=\left(\boldsymbol{v}_{1} \boldsymbol{v}_{2} \cdots \boldsymbol{v}_{n}\right)$, and assume that $\tilde{\boldsymbol{x}}$ is a solution of

$$
\begin{aligned}
A \boldsymbol{x} & =\boldsymbol{b} \\
\boldsymbol{x} & \geq \mathbf{0} .
\end{aligned}
$$

If the support of $\tilde{\boldsymbol{x}}$ has size at least $d+1$, then $A \boldsymbol{x}=\mathbf{0}$ has some nontrivial solution $\boldsymbol{z}$ with support contained in the support of $\tilde{\boldsymbol{x}}$. For an adequate value of $t$, the vector $\tilde{\boldsymbol{x}}+t \boldsymbol{z}$ is a solution of system (3.1) with smaller support than $\tilde{\boldsymbol{x}}$. A closer examination of this argument yields that, in the plane, any point in the convex hull of four points lies in two of the triangles they span (as illustrated on the right). The following strengthening of Carathéodory's theorem will be useful in optimization.

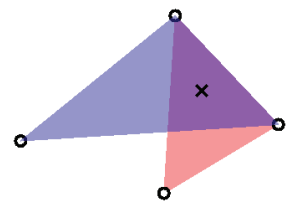

Proposition 3.1. Any point in the convex hull of (at least) $d+2$ points in $\mathbb{R}^{d}$ lies in the convex hull of at least two $(d+1)$-element subsets of these points. 


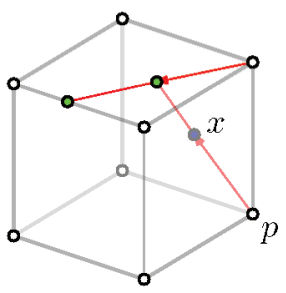

Let us give a geometric proof of Proposition 3.1 for $d+2$ points. Let $p$ be any one of the $d+2$ points, and let $x$ be in the interior of their convex hull. Shoot a ray from $\boldsymbol{p}$ to $\boldsymbol{x}$ and collect the (at most) $d$ vertices of the face of a (triangulation of the) convex hull through which this ray exits; these $d$ points and $\boldsymbol{p}$ contain the point $\boldsymbol{x}$ in their convex hull, and any of the $d+2$ points can be used as the origin of the ray.

The figure above illustrates this process in the case of a (blue) point $\boldsymbol{x}$ contained in the convex hull of the (white) vertices of a cube in $\mathbb{R}^{3}$. The green projections to faces help us find the four white points containing $\boldsymbol{x}$. Several variants of Carathéodory's theorem have been developed. For instance, Steinitz 364 proved that a point in the interior of the convex hull of a set $S$ lies in the interior of the convex hull of some $2 d$ points of $S$. A related classical result is the Krein-Milman theorem 251]: if $C$ is a convex set, then every point in $C$ is a convex combination of its extreme points, i.e., those that are not convex combinations of others in the set. Allowing more flexible representations, Dobbins 141 proved that any point in an $a b$-dimensional polytope is the barycenter of $a$ points on its $b$-dimensional skeleton (another proof of Dobbins's theorem is shown in [67]).

One of the most applicable and powerful variants is the colorful Carathéodory theorem. We saw this in the introduction already.

Colorful Carathéodory theorem. Let $C_{1}, C_{2}, \ldots, C_{d+1}$ be point sets in $\mathbb{R}^{d}$. If a point $\boldsymbol{p}$ is in the convex hull of every $C_{i}$, then there exist $\boldsymbol{x}_{1} \in C_{1}, \boldsymbol{x}_{2} \in C_{2}, \ldots$, $\boldsymbol{x}_{d+1} \in C_{d+1}$ such that $\boldsymbol{p}$ lies in the convex hull of $\left\{\boldsymbol{x}_{1}, \boldsymbol{x}_{2}, \ldots, \boldsymbol{x}_{d+1}\right\}$.

In other words, if the origin is contained in the convex hull of each of $d+1$ point sets $C_{1}, C_{2}, \ldots, C_{d+1}$ (the color classes), then it is contained in a colorful simplex, i.e., one where each of the vertices comes from a different $C_{i}$ (with the understanding that this simplex may be degenerate). This theorem was discovered by Bárány [40, who showed that a colorful simplex that minimizes the distance to the origin must contain it. Indeed, when the distance is still positive, it is attained on a facet and the vertex opposite to that facet may be changed to further decrease the distance to the origin. This approach inspired new proofs and algorithms, by minimization, of the colorful Carathéodory theorem [46] and other results such as Tverberg's theorem 329, 377,378, (see more on Tverberg's theorem later in Section 3.3 .

An alternative proof of the colorful Carathéodory theorem applies Meshulam's lemma (Proposition 2.6) to the join of two abstract simplicial complexes built on top of $\bigcup_{i=1}^{d+1} C_{i}$ : one has a simplex for any subset of points with no repeated color, and the other has a simplex for every subset of points not surrounding the origin. (The labeling is given by the identification of the vertices of the join to $\bigcup_{i=1}^{d+1} C_{i}$.) This approach emerged from a colorful Helly theorem of Kalai and Meshulam [222] (see below) and later allowed a purely combinatorial generalization of the colorful Carathéodory theorem by Holmsen [209], where the geometry and the colorfulness are abstracted away into, respectively, an oriented matroid and a matroid. See also the paper [212]. 
The assumption of the colorful Carathéodory theorem ensures that not only one, but actually many colorful simplices exist; we come back to this question when discussing simplicial depth in Section 7.4. This also underlies its connection to Tverberg's theorem, which we discussed in Section 3.3 .

Many variations and strengthenings of the colorful Carathéodory theorem have been explored, starting with Bárány's seminal paper [40] and other collaborations [46]. Recent strengthenings include Deza et al. colorful simplicial depth [139] (discussed in Section 7.4) and Frick and Zerbib's common generalization of the colorful Carathéodory theorem and the KKM theorem [172. Another key variation, discovered independently by Arocha et al. [29] and Holmsen et al. [210], is that the assumption that the convex hull of each $C_{i}, 1 \leq i \leq d+1$, contains the origin can be weakened to only require that the convex hull of each pair $C_{i} \cup C_{j}, 1 \leq i<j \leq d+1$, contains the origin. There are examples showing that it is not sufficient that the convex hulls of triples contain the origin, but weaker relaxations are possible [275]. Arocha et al. 29] also proved another "very colorful Carathéodory theorem".

Via point-hyperplane duality, one can derive a colorful theorem of Helly from the colorful Carathéodory theorem. We will not discuss this in detail, but let us explain the basics. Consider $d+1$ families $F_{1}, F_{2}, \ldots, F_{d+1}$ of convex sets inside $\mathbb{R}^{d}$ (researchers think of these as colors). Assume that every colorful selection of $d+1$ of the sets (i.e., one set from each $F_{i}$ ) has a nonempty common intersection. Then, the classical colorful Helly theorem of Lovász (see [40]) says that there is at least one family $F_{i}$, whose sets have a nonempty intersection. Here is now the dual of the very colorful Carathéodory theorem of [29]: given a finite family of halfspaces in $\mathbb{R}^{d}$ colored with $d+1$ colors, if every colorful selection of $d+1$ halfspaces has a nonempty common intersection, then there exist two color classes, all of whose members intersect. For more examples and references, see 253] and references therein.

The proof of the colorful Carathéodory theorem also implies that given $d+1$ point sets $C_{1}, \ldots, C_{d+1}$ and a convex set $C$, either one $C_{i}$ can be separated from $C$ by a hyperplane or there exists a colorful simplex intersecting $C$. Building on this, Mustafa and Ray [295] showed that given $\left\lfloor\frac{d}{2}\right\rfloor+1$ sets of points in $\mathbb{R}^{d}$ and a convex object $C$, then either one of the sets can be separated from $C$ by a constant number of hyperplanes or there is a $\left\lfloor\frac{d}{2}\right\rfloor$-dimensional colorful simplex intersecting $C$.

The integer Carathéodory problem considers a finite set $X \subset \mathbb{Z}^{d}$ and $\boldsymbol{v} \in \mathbb{Z}^{d}$ in its positive hull and asks whether $\boldsymbol{v}$ can be written as a nonnegative, integer linear combination of some elements of $X$, and, if true, how many elements are needed. The answer to the first question is negative in general. For instance consider

$$
X=\left\{\left(\begin{array}{l}
1 \\
0
\end{array}\right),\left(\begin{array}{l}
1 \\
2
\end{array}\right)\right\} \quad \text { and } \boldsymbol{v}=\left(\begin{array}{l}
1 \\
1
\end{array}\right)
$$

where $\boldsymbol{v}$ is an integral vector of the positive hull of $X$ but is not an integer nonnegative combination of elements of $X$.

It is thus natural to restrict one's attention to subsets $X \subset \mathbb{Z}^{d}$ such that every integral point of the cone of $X$ can be written as a nonnegative integer combination of elements of $X$; such sets are called Hilbert generating sets. This restriction is reasonable given that the integer points of any rational polyhedral cone $C$ have a finite Hilbert generating set. However, even in this setting, there is no version of an integer Carathéodory theorem with a bound on the size of the representation 
depending only on the dimension. Take for example

$$
\begin{gathered}
X_{n}=\left\{2^{i} \boldsymbol{e}_{j}+\boldsymbol{e}_{d}: 0 \leq i \leq n-1 \text { and } 1 \leq j \leq d-1\right\} \subset \mathbb{Z}^{d} \text { and } \\
\boldsymbol{v}_{n}=\left(2^{n}-1,2^{n}-1, \ldots, 2^{n}-1, n(d-1)\right)^{T},
\end{gathered}
$$

where $\boldsymbol{v}_{n}$ can be written as an integer combination over $X_{n}$, but any such combination requires at least $n$ summands. Notice that the coefficients in example (3.3) grow quickly with $n$. Such growth is necessary to force larger and larger sums because a Carathéodory-type theorem is possible if one wants to bound the number of summands in terms of the dimension and the size of the coordinates. The best upper bound in that direction was recently obtained in [14]. An earlier bound appears in [155].

Theorem 3.2. Let $X=\left\{\boldsymbol{x}_{1}, \ldots, \boldsymbol{x}_{t}\right\} \subseteq \mathbb{Z}^{d} \backslash\{\mathbf{0}\}$ be a finite set, let $\|X\|_{\infty}=$ $\max _{\boldsymbol{x} \in X}\|\boldsymbol{x}\|_{\infty}$, let $W=\left(\boldsymbol{x}_{1} \cdots \boldsymbol{x}_{t}\right)$, and let $\Lambda$ denote the sublattice of $\mathbb{Z}^{t}$ of the integer points in the row space of $W$. Any vector representable as nonnegative integer combination over $X$ can be written as a combination of at most $\min \{\operatorname{rank} W+$ $\left.\log \operatorname{det}(\Lambda), 2 d \log \left(2 \sqrt{d}\|X\|_{\infty}\right)\right\}$ terms.

The proof of Theorem 3.2 starts from some nonnegative integer combination and uses some element of the kernel of $W$ to eliminate one of the summands. This is very similar to the classical proof of the real-valued Carathéodory theorem, but now the kernel element must be an integral vector with coordinate entries in $\{-1,1,0\}$. That such a vector exists is no longer a rank argument but follows from Siegel's lemma (see [352,379]).

Interestingly, a full-fledged integer Carathéodory theorem, depending only on the dimension, does exist for Hilbert bases of pointed cones. Let us explain. First of all, a Hilbert basis is an inclusion-minimal Hilbert generating set. We say a cone is pointed if it contains no linear subspace other than the nullspace. It is known that a pointed cone has a unique Hilbert basis; see, e.g., [124, Corollary 2.6.4]. In contrast, when the cone is not pointed, there is no uniqueness, for instance $\{(x, y): x+y=0\}$ has two Hilbert bases $\{(1,-1),(-1,1)\}$ and $\{(2,-2),(-1,1)\}$. As we will see in Sections 6.2.2 and 6.3.2. Hilbert bases play an important role in optimization theory and in the solution of integer optimization problems. Here is the best-known upper bound of the number of Hilbert basis elements necessary to write a vector. This is due to Sebö [347].

Theorem 3.3. If the pointed cone $C$ is generated by a Hilbert basis $X \subseteq \mathbb{Z}^{d}$, then any of its integral points can be written as a nonnegative integer combination of at most $2 d-2$ elements of $X$.

A weaker version of Sebö's theorem, with a constant $2 d-1$, was previously obtained by Cook, Fonlupt, and Schrijver [115]. Note that the sets $X_{n}$ in example (3.3) do define pointed cones but are not the Hilbert bases of those cones. Sebő's theorem gives an upper bound, but the best-known lower bound on the size of the linear combination is only $d+\left\lfloor\frac{d}{6}\right\rfloor$ for $d \geq 6[83]$, so that leaves an open important problem:

Open Problem 3.4. Determine the best-possible constant for the integer Carathéodory theorem on Hilbert bases of pointed cones.

The answer is known to be $d$ for $d=3$ [347] and in some special cases such as the cone formed by the bases of any matroid 184. 
We conclude with an approximate Carathéodory theorem, recently recovered by Barman [51, which has an interesting application in game theory (see Section 4.1.2). Informally, it says that any point in the convex hull of a point set $X \subseteq \mathbb{R}^{d}$ can be approximated by a convex combination of few elements of $X$. The precise relation between the quality of approximation and the size of the convex combination is quantified as follows.

Theorem 3.5. Let $p \in[2, \infty)$, and let $X \subseteq \mathbb{R}^{d}$. For any point $\boldsymbol{a} \in \operatorname{conv}(X)$ and any $\varepsilon>0$, there is a point $\boldsymbol{b}$ such that $(i)\|\boldsymbol{a}-\boldsymbol{b}\|_{p} \leq \varepsilon$, and (ii) $\boldsymbol{b}$ can be expressed as a linear combination of at most $4 p\left(\frac{\max _{\boldsymbol{x} \in X}\|\boldsymbol{x}\|_{p}}{\varepsilon}\right)^{2}$ vectors from $X$.

Observe that the number of points used to represent the approximation $\boldsymbol{b}$ to $\boldsymbol{a}$ is independent of the ambient dimension $d$. The point $\boldsymbol{b}$ can in fact be chosen as the barycenter of points of $X$ with nonnegative integer weights, the sum of the weights being at most $k=4 p\left(\frac{\max _{\boldsymbol{x} \in X}\|\boldsymbol{x}\|_{p}}{\varepsilon}\right)^{2}$. Barman's nice probabilistic proof writes $a$ as a barycentric combination of $d+1$ points of $X$ (by Carathéodory's theorem) and finds the $k$ points adding to $\boldsymbol{b}$ by sampling those $d+1$ points using the weights as probabilities (some special care must be taken to ensure a bound independent of the dimension). Theorem 3.5 can also be derived from Maurey's lemma in functional analysis (see Pisier [316] and Carl [90]). See also [72] for the derivation of a related theorem using the perceptron algorithm [305. A very recent new generalization of Theorem [3.5 was presented by Adiprasito, Bárány, and Mustafa in [3]. They proved that, given a point set $P \subset \mathbb{R}^{d}$ of cardinality $n$, a point $\boldsymbol{a} \in \operatorname{conv}(P)$, and an integer $r \leq d, r \leq n$, then there exists a subset $Q \subset P$ of $r$ elements such that the distance between $\boldsymbol{a}$ and $\operatorname{conv}(Q)$ is less then $\operatorname{diam} P / \sqrt{2 r}$. Here the diameter of $P$ is the largest distance between a pair of points in $P$.

3.2. Helly. Helly's theorem asserts that for a finite family of convex subsets of $\mathbb{R}^{d}$ with at least $d+1$ members, if every $d+1$ member intersects, then the whole family intersects. In the contrapositive, the empty intersection of finitely many convex sets in $\mathbb{R}^{d}$ is always witnessed by the empty intersection of some $d+1$ of the sets; see Figure 8

The special case of Helly's theorem where each subset is a halfspace is of particular interest. Since a family of halfspaces not containing the origin has empty intersection if and only if their inner normals positively span the space, Helly's theorem for halfspaces is equivalent to Carathéodory's theorem for their polars. Note
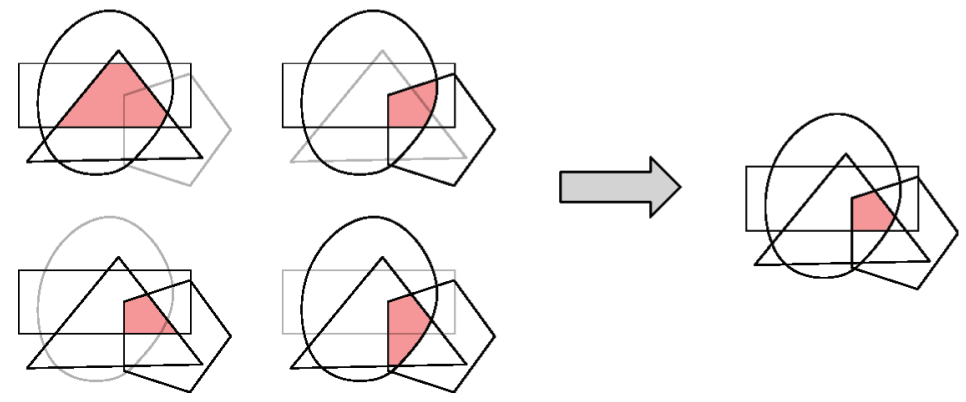

FiguRE 8. Helly's theorem in the plane 
that the polar of a set of hyperplanes is a set of ray vectors. The case of halfspaces implies the general case because, given a family of convex sets in $\mathbb{R}^{d}$, we can replace each set by a polytope that it contains without altering the intersection patterns. It suffices to take a witness point in the intersection of every subfamily and then replace each set by the convex hull of the witness points that it contains.

Helly's original proof used the separability of compact convex sets by hyperplanes to set up an induction on the dimension. It is spelled out in the survey of Danzer et al. 120, Section 1] along with references to eight other proofs. The most common proof deduces Helly's theorem from Radon's lemma (the case $r=2$ of Tverberg's theorem) [257, Section 1.3]. Starting with $k \geq d+2$ convex sets $C_{1}, C_{2}, \ldots, C_{k}$ where any $k-1$ intersects, one picks a witness point $\boldsymbol{w}_{i} \in \bigcap_{j \neq i} C_{j}$. Partition $\boldsymbol{w}_{1}, \boldsymbol{w}_{2}, \ldots, \boldsymbol{w}_{k}$ into two subsets with intersecting convex hulls, and observe that this intersection point lies in every convex set. A proof of Helly's theorem, due to Krasnoselsky [236], fits well with the theme of our survey as it uses the KKM theorem. Lift the $\boldsymbol{w}_{i}$ to the vertex $\boldsymbol{e}_{i}$ of the simplex $\Delta_{k-1}$. The map $\boldsymbol{e}_{i} \mapsto \boldsymbol{w}_{i}$ extends into a linear map $f: \Delta_{k-1} \rightarrow \mathbb{R}^{d}$, and setting $D_{i}=f^{-1}\left(\operatorname{conv}\left\{\boldsymbol{w}_{j}: j \neq i\right\}\right)$ produces $k$ closed subsets of $\Delta_{k-1}$. Each facet of $\Delta_{k-1}$ is covered by a distinct $D_{i}$ and the $D_{i}$ 's cover $\Delta_{k-1}$ by Carathéodory's theorem in $\mathbb{R}^{d}$. The KKM theorem (see Section 2.2) yields a point $\boldsymbol{w} \in \bigcap_{i} D_{i}$ and the point $f(\boldsymbol{w})$ is contained in every $C_{i}$. Chakerian showed in 92 that Helly's theorem also follows from Brouwer's fixed point theorem, in a similar fashion as the proof of the KKM theorem: the function, instead of moving every point $\boldsymbol{x}$ by the vector of its distances to each $C_{i}$, moves every point $\boldsymbol{x}$ to the barycenter of its projections on each $C_{i}$. (See the picture at right for an illustration: the projections of $\boldsymbol{x}$ to the three convex sets, red, green, and blue, are shown by the white points; the black square is their barycenter).

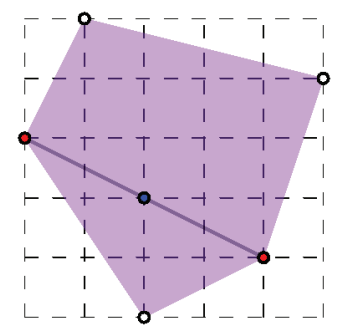

Helly's theorem for integral points was first established by Doignon [143, Proposition 4.2] and rediscovered later by Scarf [340] and Bell [56]. Hoffman [208] observed that the techniques apply for more cases than the integer lattice. The proofs of Doignon, Bell, and Hoffman hinge on the following insight: if a polytope in $\mathbb{R}^{k}$ has $m$ vertices, each with integer coordinates, and it contains no other integral point, then no two vertices may have all their coordinates of the same parity (else their midpoint would yield a contradiction), and thus $m$ is at most $2^{k}$. Bell's proof starts with a family of $m$ halfspaces in $\mathbb{R}^{k}$ whose intersection contains no integer point and such that removing any halfspace would enlarge the intersection to include some integer point. Translating each halfspace in the direction of its outer normal until every facet contains a witness point with integer coordinates, one gets witness points that must be distinct and form a polytope as above, so $m$ is at most $2^{k}$. Hoffman's proof is more complicated but holds in a more general axiomatic setting. Scarf's proof is algorithmic and relies on Sperner's lemma (see also the variation by Todd [373]). We remark that the equivalence via polarity between Helly's and Carathéodory's theorems in $\mathbb{R}^{d}$ does not carry over to $\mathbb{Z}^{d}$, as the bounds are respectively $2^{d}$ and at most $2 d-2$ (by Theorem 3.3 ). 
Some of our applications will use the following version where some of the coordinates in the intersection may be required to be integers.

Mixed Helly theorem. Let $\mathcal{F}$ be a finite family of convex sets in $\mathbb{R}^{d+k}$ of cardinality at least $(d+1) 2^{k}$. If every $(d+1) 2^{k}$ members of $\mathcal{F}$ have a common point whose last $k$ coordinates are integer numbers, then all members of $\mathcal{F}$ have an intersection point whose last $k$ coordinates are integer numbers.

The mixed Helly theorem was announced by Hoffman [208] as one of the outcomes of his axiomatic setting; however, he deferred details of the proof for the mixed analogue of the property of the Doignon theorem to a forthcoming paper, which never appeared. A complete proof came decades later and is due to Averkov and Weismantel [36. Their proof proceeds in two steps. They start with a family of halfspaces in $\mathbb{R}^{d+k}$ whose intersection is a nonempty full-dimensional polytope $P$ containing no point whose last $k$ coordinates are integers (the general case follows). They project $P$ onto the last $k$ coordinates, obtaining a polytope $T(P)$ in $\mathbb{R}^{k}$ with no integer point; Doignon's theorem then ensures that at most $2^{k}$ of the halfspaces supporting the facets of $T(P)$ already intersect with no integer point. By Carathéodory's theorem in $\mathbb{R}^{d+k}$, each $k$-dimensional halfspace is the projection of the intersection of some at most $d+1$ of the original halfspaces; the bound follows.

Fractional versions of Helly's theorem play an important role in the study of sampling and hitting geometric set systems. Here, fractional means that one only assumes that some constant fraction of the subfamilies (of a given size) intersect, and one concludes that some constant fraction of the whole family intersect.

Theorem (Fractional Helly theorem). Let $0<\alpha \leq 1$, and let $\mathcal{F}$ be a family of $n$ convex sets in $\mathbb{R}^{d}$. If $\alpha\left(\begin{array}{c}n \\ d+1\end{array}\right)$ of the $(d+1)$-element subsets of $\mathcal{F}$ have nonempty intersection, then some $\left(1-(1-\alpha)^{\frac{1}{d+1}}\right) n$ elements of $\mathcal{F}$ intersect.

The first result in this direction was proven by Katchalski and Liu [229]. Starting with a family of $n$ convex sets, they assign to any subfamily the lexicographically minimum point in their intersection. The set of points lexicographically larger than a given point is convex, so Helly's theorem ensures that the minimal point of the intersection of $k \geq d$ convex sets is also the minimal point in the intersection of some $d$ among them. A weak version of the above theorem, where the size of the intersecting subfamily is only guaranteed to be at least $\frac{\alpha}{d+1} n$, then follows from a pigeonhole argument.

There are few settings in which fractional Helly theorems are known. On the one hand, Matoušek 260] proved, via a general sampling technique due to Clarkson [109, 113, that any set system with bounded VC dimension affords a fractional Helly theorem; his approach holds for other measures of complexity than the VC dimension [315]); we come back to the notions of VC dimension in Section 7.2. On the other hand, Bárány and Matoušek [45] established a fractional Helly theorem for lattices, including over the integers. It is surprising that they only have to check the nonempty integral intersection of a positive fraction of $(d+1)$-tuples, instead of the expected $2^{d}$-tuples of intersections.

The bound of $\left(1-(1-\alpha)^{\frac{1}{d+1}}\right) n$ in Theorem 3.2 is sharp and was obtained by Kalai [217] and, independently, by Eckhoff [151, via a study of nerve complexes that led to a more general topological point of view. The nerve of a family of convex sets is the abstract simplicial complex with a vertex for every set in the 
family, and a simplex for every intersecting subfamily. Helly's theorem and its fractional version easily translate in terms of nerves: the former states that the nerve cannot contain the boundary of a $(\geq d)$-dimensional simplex without containing the simplex, and the latter asserts that if the nerve contains a positive fraction of the $d$-dimensional faces, then it must contain a simplex of dimension a positive fraction of $n$. Kalai's proof uses his technique of algebraic shifting [220] to study how the number of simplices of various dimensions behaves as the nerve is simplified through a sequence of $d$-collapses, a type of filtration available to nerves of convex sets [372]. If a complex is $d$-collapsible, then all its subcomplexes have trivial homology in dimension $d$ and above, i.e., it is $d$-Leray. Kalai's proof of Theorem 3.2 extends to $d$-Leray complexes (see [205, §5.2]), and Alon et al. [21] further proved that families of subsets of $\mathbb{R}^{d}$ that are closed under intersection and whose nerve is $d$ Leray also admit weak $\varepsilon$-nets and $(p, q)$-theorems; examples of such families include good covers (this follows from the nerve theorem [75]) and acyclic covers [135. Topological versions of Helly's theorem have a further application in geometric group theory [162].

Fairly general topological Helly theorems can be derived from nonembeddability results via a construction reminiscent, again, of the setup of the KKM theorem. Let us illustrate the basic idea with five sets in the plane. If any four intersect and any three have path-connected intersection, then we can draw $K_{5}$, the complete graph on five vertices, inside the family by placing each vertex in the intersections of four sets (different vertices missing different sets) and connecting any two vertices by a path contained in the intersection of the three sets that contain them both. By a classical theorem of Kuratowski for planar graphs 140, there exist two edges that have no common vertex and that intersect. This intersection point must be in all five sets. An induction on the same idea yields that in a family of planar sets, where intersections are empty or path connected, if every four sets intersect, then they all must intersect. In higher dimensions, where all graphs can be drawn without crossing, the same approach can be combined with nonembeddability results derived from the Borsuk-Ulam theorem, e.g., the Van Kampen-Flores theorem which states that $\Delta_{2 k+2}^{(k)}$ does not embed in $\mathbb{R}^{2 k}$ [165, 381]; cf. [258, Chapter 5]. The discussion we present below on the topological Tverberg theorem is also connected to embeddability of complexes; e.g., the paper [66] proves that the topological Radon theorem implies the Van Kampen-Flores theorem.

The most general result in this direction [188] is that any family $\mathcal{F}$ of subsets in $\mathbb{R}^{d}$ admits a Helly-type theorem in which the constant that replaces $d+1$ in the case of convex sets is bounded as a function of the dimension $d$ and

$$
b=\max _{G \subseteq \mathcal{F}, 0 \leq i \leq\left\lceil\frac{d}{2}\right\rceil-1} \tilde{\beta}_{i}(\bigcap G),
$$

where $\tilde{\beta}_{i}(X)$ denotes the $i$ th reduced Betti numbers, over $\mathbb{Z}_{2}$, of a space $X$.

Nonembeddability arguments and the study of nerves offer two different pathways to topological Helly theorems. While the former allows more flexible assumptions, the latter offers more powerful conclusions in the form of a sharp fractional Helly theorem. It is not known whether the benefits of both approaches could be combined.

Open Problem 3.6. Given $b$ and $d$, is there a fractional Helly theorem for families $\mathcal{F}$ of subsets in $\mathbb{R}^{d}$ where $\tilde{\beta}_{i}(\bigcap G) \leq b$ for any $G \subseteq \mathcal{F}$ and any $0 \leq i \leq\left\lceil\frac{d}{2}\right\rceil-1$ ? 
This open question relates to a more systematic effort to build a theory of homological VC dimension [221, Conjectures 6 and 7]. There are some recent results for planar sets with connected intersections (the case $d=2$ and $b=0$ ) [213.

There are too many Helly-type theorems to list in this survey, but we wish to point out at least one more variation. Quantitative Helly theorems were introduced by Bárány, Katchalski, and Pach in 43. In this family of Helly-style theorems one is not content with a nonempty intersection of a family, but the intersections must have measurable or enumerable information in the hypothesis and the conclusion. Typical measurements include the volume, the diameter, or the number of points in a lattice. Motivated by applications in optimization, in the last two years several papers have been published on this subject, both for continuous [80, 127, 303] and for discrete [12, 35, 106, 326] quantitative Helly-type theorems. For other recent Helly-type theorems see [28].

We next discuss the fifth remarkable theorem of our survey.

3.3. Tverberg. Tverberg-type theorems allow for the partition of finite point sets so that the convex hulls of the parts intersect. In its original form we have the following.

Tverberg theorem. Any set of at least $(r-1)(d+1)+1$ points in $\mathbb{R}^{d}$ can be partitioned into $r$ subsets whose convex hulls all have at least one point in common.

Such a division in parts is often called a Tverberg partition; see Figure 9 The case $r=2$ is known as Radon's lemma [321, and the case $d=2$, but with general $r$, was proven by Birch [61] before Tverberg proved the general statement.

Tverberg's first proof of his theorem [376] relies on a deformation argument: start with a configuration with a known Tverberg partition, and move the points continuously to the target configuration. This process is such that, while the number of Tverberg partitions may change, there will always be one present. A simpler proof consists in arguing that a partition of the point set minimizing an adequate function must be a Tverberg partition. This idea, which originates in Bárány's proof of the colorful Carathéodory theorem, was gradually refined by Tverberg [377, Tverberg and Vrećica 378, and Roudneff [329] (Roudneff minimizes the sum of the squared distances between a point and the convex hulls of the parts). Another proof, due to Sarkaria [334, uses multilinear algebra to deduce Tverberg's theorem from the colorful Carathéodory theorem; the idea behind Sarkaria's proof was later made simpler (using explicit tensors instead of number fields) and more algorithmic by Bárány and Onn in 47. Recently, Bárány and Soberón revisited these ideas and proved a new generalization of Tverberg's theorem using affine combinations [49].

Just as for Carathéodory's and Helly's theorems, there is an integer Tverberg theorem. Its most recent version guarantees that any set of at least $(r-1) d 2^{d}+1$
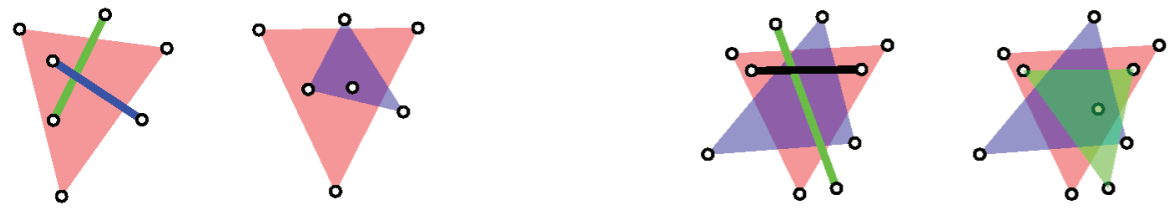

Figure 9. Tverberg's theorem in the plane: the two types of partitions for $r=3$ (left) and for $r=4$ (right). 

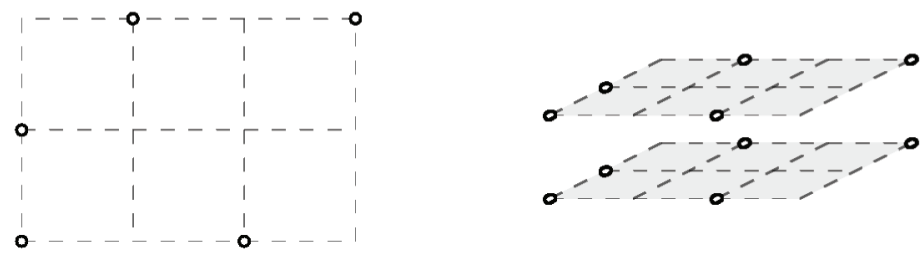

Figure 10. The lower bound for the integral Radon theorem. Left: Five integral points in the plane with no integral Radon partition. Right: A configuration of $k$ integral points in $\mathbb{R}^{d}$ with no Radon partition can be turned into a configuration of $2 k$ integral points in $\mathbb{R}^{d+1}$ with no Radon partition.

integer points in $\mathbb{Z}^{d}$ can be partitioned into $r$ parts whose convex hulls have a point of $\mathbb{Z}^{d}$ in common 130 . The proof of this upper bound goes as follows. From the integer Helly theorem, one can prove that any finite set of integer points $S \subset \mathbb{Z}^{d}$ has an integer centerpoint: a point $\boldsymbol{p}$ such that for every hyperplane $H$ containing $\boldsymbol{p}$, one of its halfspaces contains at least $|S| / 2^{d}$ points from $S$. Using this centerpoint, it is not difficult to see that $(r-1) d 2^{d}+1$ points can be partitioned into $r$ pairwise disjoint simplices all containing $\boldsymbol{p}$. We will see more about centerpoints in Sections 6.5 .5 and 7.4 .

The upper bound on the integer Tverberg number is not known to be sharp, and the best lower bound of $2^{d}(r-1)+1$ is due to Doignon (this result was communicated in [153]). Recently in 125 the authors showed that the Tverberg number in $\mathbb{Z}^{2}$ is exactly $4 r-3$ when $r \geq 3$, and they improved the upper bounds for the Tverberg numbers of $\mathbb{Z}^{3}$.

The special case of bipartition, i.e., $r=2$, is called the integral Radon theorem. A sharper upper bound of $d 2^{d}-d+3$ and a lower bound of $\frac{5}{4} 2^{d}+1$ were established by Onn [308, (see Figure 10). Even low-dimensional cases are hard. The only sharp bound known, also due to Onn, is for $r=d=2$ : any six integral points in the plane have an integral Radon partition. An upper bound of 17 for the case $d=3$ was proven by Bezdek and Blokhuis [60.

Open Problem 3.7. Determine the exact value of the integer Tverberg numbers. In particular, is the integer Radon number for $d=3$ less than 17? Is it bigger than 11 ?

More generally, there is the notion of an integer quantitative Tverberg number: any set of at least $\left(\left(2^{d}-2\right)\left[\frac{2}{3}(k+1)\right]+2\right)(r-1) k d+k$ integer points can be partitioned into $r$ parts whose convex hulls have $k$ integral points in common [130]. Similar results hold more generally for sets that are discrete (i.e., the sets intersect any compact in only finitely many points), for instance, the difference between a lattice and one of its sublattices. Recent improvements on the quantitative integer Helly theorem [35,106] leads to sharper upper bounds for the Tverberg version.

Open Problem 3.8. Determine tighter lower and upper bounds on the integer quantitative Tverberg numbers.

Let $\Delta_{(r-1)(d+1)}^{(d)}$ denote the $d$-dimensional skeleton of the simplex with $(r-1)(d+1)+1$ vertices. Tverberg's theorem can be understood as stating that for any linear map from $\Delta_{(r-1)(d+1)}^{(d)}$ into $\mathbb{R}^{d}$ there must exist $r$ disjoint simplices whose images 

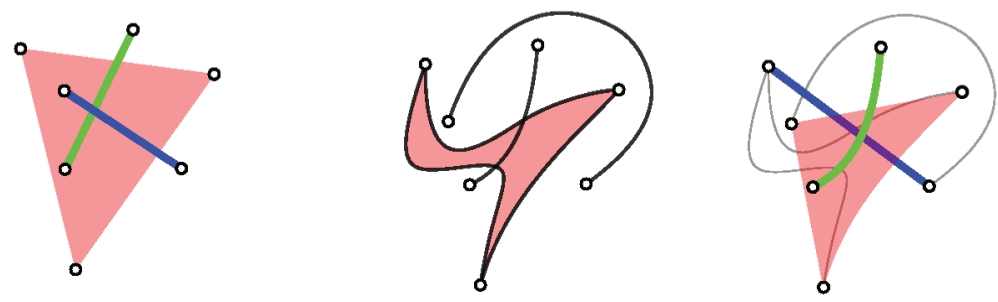

Figure 11. The topological Tverberg theorem in the plane $(r=3)$. Left: A configuration of seven points and its Tverberg partition into three parts. Center: The linear map used in the left is deformed continuously so as to break the previous Tverberg partition, every edge not represented is kept straight. Right: Nonetheless, a new Tverberg partition emerges.. Note that the topological Tverberg conjecture remains open in dimension two when $r$ is not a prime-power. See 42 and, for the two-dimensional case, 342].

intersect. This reformulation invites the question, going as far back as 1979 (see ending of the important paper [39]), whether the same conclusion holds for all continuous maps. In other words, is there a topological Tverberg theorem? For $r=2$, this is the question of nonembeddability discussed above in relation to topological Helly theorems; see Figure 11.

Positive answers were obtained first for $r=2$ (the topological Radon theorem) by Bajmóczy and Bárány [39, then for $r$ prime by Bárány et al. [48, and for $r$ a power of a prime by Özaydin [310] and independently, but later, by Volovikov [386] and Sarkaria [335]. Matoušek [258, Chapters 5 and 6] offers an accessible introduction to the techniques behind the topological Tverberg theorem.

For $r=2$, the proof of the topological Radon theorem uses the notion of deleted product of a geometric simplicial complex $\mathrm{K}$ with itself, defined as

$$
\mathrm{K}_{\Delta}^{2}=\{\sigma \times \tau: \sigma, \tau \in \mathrm{K}, \sigma \cap \tau=\varnothing\} .
$$

Now, for contradiction, suppose there exists a continuous map $f:|\mathrm{K}| \rightarrow \mathbb{R}^{d}$ with the property that points in distinct faces are mapped to distinct points. It induces another continuous map $\tilde{f}:\left|\mathrm{K}_{\Delta}^{2}\right| \rightarrow \mathbb{S}^{d-1}$ where $\tilde{f}\left(x_{1}, x_{2}\right)=\frac{f\left(x_{1}\right)-f\left(x_{2}\right)}{\left\|f\left(x_{1}\right)-f\left(x_{2}\right)\right\|}$. The map $\tilde{f}$ commutes with the central symmetries of $\mathbb{S}^{d-1}$ and $\mathrm{K}_{\Delta}^{2}$, where the central symmetry of $\mathrm{K}_{\Delta}^{2}$ is the exchange of the two components. When $\mathrm{K}$ is the boundary of the $d$-dimensional simplex, $\mathrm{K}_{\Delta}^{2}$ is homotopy equivalent to $\mathbb{S}^{d}$ (this is not trivial). Since the Borsuk-Ulam theorem prevents the existence of an antipodal map from $\mathbb{S}^{d}$ to $\mathbb{S}^{d-1}$, the continuous $f$ will have two faces intersecting in its image, which gives us a contradiction.

More generally, to prove the topological Tverberg theorem when $r$ is a prime, one may start with a map from $\mathrm{K}=\Delta_{(r-1)(d+1)}^{(d)}$ to $\mathbb{R}^{d}$ with no $r$-wise intersection and use it to build another map from an $r$-fold deleted product $\mathrm{K}_{\Delta}^{r}$, replace the antipodality by the action of the symmetric group, and apply a generalization of the Borsuk-Ulam theorem such as Dold's theorem [144. The case of $r$ a prime power is technically more involved. (Note that this outline leaves out some issues such as dimension-reduction considerations [342.) 
By the late 1990s, the widespread belief that Bárány's question had a positive answer for every $r$ and $d$ was known as the topological Tverberg conjecture. It was only recently refuted by Frick [168, who completed an approach of Mabillard and Wagner 252] building on Özaydin's work 310. In a nutshell, Özaydin proved that an equivariant map from the adequate $r$-fold product $\tilde{X}$ exists if and only if $r$ is not a prime power. Mabillard and Wagner proposed an isotopy-based approach to construct a map with no $r$-wise intersection when such an equivariant map exists, but they could only develop it in codimension larger than what the topological Tverberg conjecture allows. Frick overcame this codimension restriction, producing the first series of counterexamples to the topological Tverberg conjecture. The current state of affairs is that a counterexample is known for every $r$ that is not a prime power and for every $d \geq 2 r$. See the survey [42] for more details.

To conclude our discussion of "all things Tverberg", let us highlight some natural variants inspired by Tverberg's theorem for which only very partial results are known. One can find the most recent variants and extensions of Tverberg's theorem in $[50,126,317$.

A tolerant Tverberg theorem, due to Soberón and Strausz [358, asserts that any set of $(t+1)(r-1)(d+1)+1$ points can be partitioned into $r$ parts such that, after deletion of any $t$ points, what remains is a Tverberg partition. This bound was improved to $r(t+2)-1$ for $d=1$ and to $2 r(t+2)-1$ for $d=2$ [285] (the bound for $d=1$ is tight). Recently there have been two significant improvements, GarcíaColín et al. 180 gave an asymptotically tight bound for the tolerant Tverberg theorem when the dimension and the size of the partition are fixed. Later, in [357, Soberón used the probabilistic method to give another asymptotic bound that is polynomial in all three parameters. Still we can ask for precise values.

Open Problem 3.9. What is the smallest number $n$ such that any set of $n$ points in $\mathbb{R}^{d}$ has a Tverberg partition into $r$ parts that tolerates the deletion of $t$ points?

A related Carathéodory-type variation of Tverberg's theorem [29] considers $r$ linear maps $f_{1}, \ldots, f_{r}$, assumes that $f_{1}(e) \cap f_{2}(e) \cap \cdots \cap f_{r}(e)$ is nonempty for every onedimensional edge $e$ of $\Delta_{(r-1)(d+1)}$, and concludes the existence of disjoint faces in the simplex $\Delta_{(r-1)(d+1)}, \sigma_{1}, \sigma_{2}, \ldots, \sigma_{r}$ of dimensions summing to $(r-1)(d+1)+1-r$ and such that $f_{1}\left(\sigma_{1}\right) \cap f_{2}\left(\sigma_{2}\right) \cap \cdots \cap f_{r}\left(\sigma_{r}\right)$ is not empty.

A conjectured relaxed version of Tverberg's theorem, due to Reay, goes as follows. Denote by $T(d, r, k)$ the minimum positive integer number $n$ such that any set of $n$ points $a_{1}, \ldots, a_{n}$ in $\mathbb{R}^{d}$ (not necessarily distinct) admits a partition into $r$ pairwise disjoint sets $A_{1}, \ldots, A_{r}$ such that any size $k$ subfamily of $\left\{\operatorname{conv}\left(A_{1}\right), \operatorname{conv}\left(A_{2}\right)\right.$, $\left.\ldots, \operatorname{conv}\left(A_{r}\right)\right\}$ has a nonempty intersection. Note that Tverberg's theorem says $T(d, r, r)=(r-1)(d+1)+1$; Reay conjectured that the Tverberg constant is tight even for smaller values of $k$ :

Conjecture 3.10. $T(d, r, k)=(r-1)(d+1)+1$ for all $2 \leq k \leq r$.

Note that if all $\operatorname{conv}\left(A_{i}\right)$ intersect, then they intersect $k$-wise, so $T(d, r, k)$ is at most $T(d, r, r)$ and in particular $T(d, r, k)$ is finite for any $k \leq r$. Moreover, Helly's theorem ensures that $T(d, r, k)=T(d, r, r)$ for every $d+1 \leq k \leq r$. The conjecture is known to hold for $d+1 \leq 2 k-1$ or $k<r<\frac{d+1}{d+1-k} k$, and some weaker bounds are known in several other cases; we refer the interested reader to [31] and the discussion therein. 
Let us take this opportunity to mention another famous conjecture of a flavor similar to Reay's conjecture. A thrackle is a graph that can be drawn in the plane in such a way that any pair of edges intersects precisely once, either at a common vertex or a transverse intersection point.

Conjecture 3.11 (Conway's thrackle conjecture). For any thrackle, the number of edges is at most the number of vertices.

The conjecture is known to hold if all edges are drawn as straight line segments [156] (it is akin to Reay's setup for $k=2$ ). We refer the interested reader to the recent progress of Fulek and Pach [175] and the discussion and references therein.

The next big open question was stated in 1979 by Sierksma (he offered an entire Dutch cheese as a prize for whoever could solve this problem). In unpublished mimeographed notes, he conjectured about the number of distinct Tverberg partitions of a set of points guaranteed to exist for $(r-1)(d+1)+1$ points in $\mathbb{R}^{d}$.

Conjecture 3.12 (Sierksma). Any set of $(r-1)(d+1)+1$ points in $\mathbb{R}^{d}$ has at least $((r-1) !)^{d}$ distinct Tverberg partitions into $r$ parts.

We do not state the lower bounds here, as they are a bit cumbersome, but lower bounds for the number of Tverberg partitions were first obtained when $r$ is prime by Vučić and Živaljević in 388. They used topological tools to settle this. Hell showed that these bounds also hold when $r$ is a prime power [206. Later Hell, without any topology, provided better bounds for the case of the plane 207.

Last, but certainly not least, the colorful Tverberg conjecture was formulated by Bárány and Larman [44] some 25 years ago, but only a few results are known today (see [50,68, 69, 399] and the references therein).

Conjecture 3.13. Let $F_{1}, F_{2}, \ldots, F_{d+1} \subset \mathbb{R}^{d}$ be sets of $r$ points each. There exists a partition of $\bigcup_{i=1}^{d+1} F_{i}$ into $r$ sets $A_{1}, A_{2}, \ldots, A_{r}$ of $d+1$ points each, such that every $A_{i}$ contains exactly one point from every $F_{j}$ and $\bigcap_{j=1}^{r} A_{j} \neq \varnothing$.

Further conjectures along the lines of the Bárány-Larman conjecture, with colorful, discrete, and quantitative flavors were formulated by De Loera et al. [130.

3.4. Computational considerations. We continue our discussion of computational issues begun in Section 2.4. We remark that the Carathéodory and Helly theorems, in their classical real-valued versions from Section 3 , are dual to each other and, essentially, if one has an algorithm to find the Carathéodory decomposition of a vector in terms of other vectors, one also has an algorithm for finding an intersection point for a family of convex sets. For Helly's theorem, one wishes to find a point in the intersection of convex sets. The problem of finding such an intersection point can be thought of as a special case of the problem of minimizing a convex function over convex sets. We will see explicit cases for this problem later in Section 6, where the convex sets are explicitly given by constraints (i.e., equations and inequalities), but for now all that we need to know is that (a) a whole range of different algorithms for solving such problems exist; (b) some of these algorithms are in fact efficient; and (c) depending on the type of input constraints (e.g., convex sets defined linear inequalities versus arbitrary constraints), one can be even more efficient [58,76]. By the convexity assumption, a local minimum is also a global 
minimum and, thanks to Helly's and Carathéodory's theorems, there are nice necessary and sufficient conditions for when we have found an optimum. We discuss more about this in Section 6 .

Convex optimization problems have been classified in levels of increased computational difficulty, and different specialized algorithms are available (e.g., least squares, linear programming, conic optimization, semidefinite programming, etc). For example, Carathéodory's theorem, in the simplest real-valued form presented in Section 3.1. can be formulated as a linear programming problem, and Helly's theorem for halfspaces is reducible to linear programming too. The good news is linear programs have efficient algorithmic solutions, both in theory and in practice 344. Still, even if we move to the most general version of a (real-valued) Helly's theorem of finite family of arbitrary convex sets, the challenge is to solve a convex programming problem that has many types of algorithms. We cannot cover them here, but we recommend [58,76] for excellent introductions to convexity algorithms and computational methods.

Compare now the good news above to the bad news involving the integer and mixed-integer versions of Carathéodory's and Helly's theorems. We are now in the realm of combinatorial, integer, and mixed-integer programming where, for the most part, the problems are not efficiently solvable. Solving combinatorial or mixed-integer optimization problems, that is, finding an optimal solution to such problems, can be a difficult task. Unlike linear programming, whose feasible region is a convex (polyhedral) set, in combinatorial problems one must search a lattice of feasible points or, in the mixed-integer case, a set of disjoint halflines or line segments to find an optimal solution. Thus, unlike linear programming, finding a global optimum to the problem requires us to relax, approximate, decompose the solution space, and sometimes we are forced to enumerate all possibilities.

As an example of the higher computational difficulty of discrete versions of Helly's and Carathéodory's theorems let us look at the problem of computing a Hilbert basis. This was a particularly simple case for the integer version of Carathéodory's theorem presented in Section 3.1] Alas, in [150] it was proved that deciding whether a given solution belongs to the Hilbert basis of a given system is co-NP-complete. Thus, even in this tame case, the integral Carathéodory property is hard to realize computationally.

We chose to highlight the colorful Carathéodory theorem because it is so general and because it can be used to prove the original Carathéodory theorem and many other existence theorems in high-dimensional discrete geometry, such as Tverberg's theorem or the centerpoint theorem (see Section 7 for details). While the original Carathéodory's theorem can be cast as a linear program and thus a solution can be implemented in polynomial time, much less is known about the algorithmic complexity of its colorful version. More precisely, the algorithmic colorful Carathéodory problem is the computational problem of finding such a colorful choice of elements as described in the theorem. Despite several efforts in the past, the computational complexity of the colorful Carathéodory problem in arbitrary dimension is still open. In [276], Meunier et al. showed that the problem lies in the complexity class PPAD.

Open Problem 3.14. What is the complexity of finding a colorful simplex under the hypotheses of the colorful Carathéodory theorem? 
This question was formulated for the first time by Bárány and Onn in [4] and there they formulated a general family of related questions that come under the name colorful linear programming.

Meunier and Sarrabezolles 277] have shown that a closely related problem is PPAD-complete: given $d+1$ pairs of points $P_{1}, \ldots, P_{d+1} \in \mathbb{Q}^{d}$ and a colorful choice that contains the origin in its convex hull, find another colorful choice of points that contains the origin in their convex hull.

Since we have no exact combinatorial polynomial-time algorithms for the colorful Carathéodory theorem, approximation iterative algorithms are of interest. This was first considered in [47, but other researchers (e.g., 286]) have approached this problem too.

Let us now speak about computational complexity of Tverberg's theorem. Sarkaria's proof of Tverberg's theorem (later simplified by Bárány and Onn [46]) gives a polynomial-time way to compute a Tverberg partitions from a colorful Carathéodory choice with the origin in its convex hull. In this way, the computational issues about Tverberg's theorem are closely connected to computational issues regarding the colorful Carathéodory theorem. Since one can calculate a Tverberg partition from a colorful selection of Carathéodory, one can show Tverberg's theorem belongs to the class PPAD. One of the simplest, yet most frustrating, aspects of Tverberg's result is that it is not clear how to find a Tverberg partition. So it is natural to ask the following.

Open Problem 3.15. Is there a polynomial-time algorithm to find a Tverberg partition when one exists? That is, given $n=(d+1)(m+1)+1$ points in $\mathbb{R}^{d}$, compute, in time polynomial in $n$, a partition into $m$ parts with intersecting convex hulls.

Since finding an $m$-Tverberg partition is an open question, approximate versions of Tverberg's theorem are of interest. Mulzer et al. 286 designed a deterministic algorithm that finds a Tverberg partition into $n / 4(d+1)^{3}$ parts in time $d^{O(\log d)} n$. This means that for every fixed dimension one can compute an approximate Tverberg point (and hence also an approximate centerpoint) in linear time. Rolnick and Soberón 325] proposed probabilistic algorithms for computing Tverberg partitions into $n / d^{3}$ parts with error probability $\epsilon$ and with time complexity that is weakly polynomial in $n, d, \log \left(\frac{1}{\epsilon}\right)$.

\section{GAMES AND FAIR DIVISION}

Mathematics and the social sciences have had rich interactions since Condorcet's seminal work on the analysis of voting systems. The relevance of (combinatorial) convexity and topology to this interdisciplinary research was first established in the 1940s-1950s through the work of Nash, von Neumann, Gale, Shapley, Scarf, and many others, and it has been confirmed in the following decades in the development of fair-division algorithms and computational social choice (see, e.g., [78,79, 117, 304 and the many references therein). This section shows how our five discrete theorems appear in these topics too.

4.1. Strategic games. Game theory studies a broad range of games that model situations where several agents collaborate or compete. Strategic games model the situations where $N$ players interact by each choosing from finitely many strategies to play and enjoy a payoff depending on the strategies chosen by all players (his or 
her choice included). Formally, each player is modeled by the pair $\left(S_{i}, u^{i}\right)$, where $S_{i}$ is a set of strategies available to him or her and where $u^{i}$ is a payoff function $S_{1} \times \cdots \times S_{N} \rightarrow \mathbb{R}$. A central theme in the theory of strategic games is the search for equilibria, where each player's choice is the best response to the other players' choices.

4.1.1. Nash equilibria. Formally, a Nash equilibrium in pure strategies is a choice of strategy for each player $s_{1} \in S_{1}, \ldots, s_{N} \in S_{N}$ such that for $i=1, \ldots, N$ and all $g \in S_{i}$

$$
u^{i}\left(s_{1}, \ldots, s_{i-1}, s_{i}, s_{i+1}, \ldots, s_{N}\right) \geq u^{i}\left(s_{1}, \ldots, s_{i-1}, g, s_{i+1}, \ldots, s_{N}\right) .
$$

Let us illustrate pure Nash equilibria with the max-cut game, where an arbitrary graph $G=(V, E)$ is fixed and each vertex $x \in V$ represents a player. Each player $x$ chooses from two strategies $S_{x}=\{1,-1\}$, and his or her payoff function is the number of neighbors of $x$ in $G$ with a different strategy:

$$
u^{x}\left(s_{1}, \ldots, s_{|V|}\right)=\mid\left\{y: x y \in E \text { and } s_{x} \neq s_{y}\right\} \mid .
$$

Any bipartition of $V$ that maximizes the number of edges between the two parts, also called maximum cut, is a pure Nash equilibrium. Indeed, if a player could strictly increase his or her payoff by switching strategy, then this switch would increase the value of the cut.

Now, unfortunately not every $N$-player game has a pure Nash equilibrium. For example, consider the matching penny game. Players Alice and Bob simultaneously select heads or tails of a coin. If the choice is the same, then Alice wins one penny and Bob loses a penny. If they choose differently, then Bob wins a penny and Alice loses a penny. Each player thus has a choice of two strategies, and the payoffs for each player can be recorded in two $2 \times 2$ matrices ( $A$ and $B$, for each player). Note that the pure strategies alone offer no Nash equilibrium as this is a winner-takes-all situation. (In the matching penny game, the payoff matrices can be put together to show $A+B=0$; this is an example of a zero-sum game, a notion to which we will come back later.)

It turns out that equilibria always exist when considering a randomized choice of the strategies. We now allow more freedom to the players by making them choose not a single strategy, but a probability distribution over all their strategies. Once choices are made, a random strategy is selected for each player from his or her distribution, and the (random) payoff is determined. Each player then wants to maximize his or her expected payoff. Formally, a mixed strategy for player $i$ is a probability distribution $m_{i}$ on the set of pure strategies $S_{i}$. For instance, in the matching penny game, this means that each player decides his or her move according to a biased coin flip and is free to choose the bias. Note that the mixed strategies include all the pure strategies as a special case too.

The set of all possible mixed strategies are the vectors that lie in the convex polytope $M=\prod \Delta_{\left|S_{i}\right|-1}$. We define a product probability measure on $S=S_{1} \times$ $\cdots \times S_{N}$ by $P_{m}(s)=\prod_{i=1}^{N} m_{i}\left(s_{i}\right)$, where $s=\left(s_{1}, s_{2}, \ldots, s_{N}\right)$. Therefore, the expected payoff for the probability distribution $P_{m}(s)$ for the $i$ th player is

$$
U^{i}\left(m_{1}, \ldots, m_{N}\right)=U^{i}(m)=\sum_{s=\left(s_{1}, s_{2}, \ldots, s_{N}\right) \in S} P_{m}(s) u^{i}(s) .
$$


The mixed strategies $m=\left(m_{1}, \ldots, m_{N}\right)$ form a Nash equilibrium in mixed strategies if for each player $i$ and for all probability distributions $p$ on $S_{i}$, modifying $m_{i}$ to $p$ does not increase the expected payoff with respect to the choices of other players, that is

$$
U^{i}\left(m_{1}, \ldots, m_{i}, \ldots, m_{N}\right) \geq U^{i}\left(m_{1}, \ldots, m_{i-1}, p, m_{i+1}, \ldots, m_{N}\right) .
$$

The literature has plenty of examples of two-player games [262, §8.1]. Thinking about three or more players is more delicate, as illustrated by Nash's three-man poker game [301, p. 293].

The existence of Nash equilibria for mixed strategies - the theorem for which John Nash received the Nobel prize - is one of the most celebrated applications of combinatorial topology, following from Brouwer's theorem; see [300,301].

Theorem 4.1 (Nash's theorem). Every $N$-player game with continuous payoff functions has at least one Nash equilibrium in mixed strategies.

Nash's original, very short, proof [300] makes strong use of the combinatorial topology and convexity arguments. It considers the set-valued function that maps each $N$-tuple of mixed strategies $m=\left(m_{1}, \ldots, m_{N}\right)$ to the set of $N$-tuples $\left(t_{1}, \ldots, t_{N}\right)$ where $t_{i}$ is a best response to $\left(m_{1}, \ldots, m_{i-1}, m_{i+1}, \ldots, m_{N}\right)$. Because the probability distributions on $S_{i}$ are the points of the simplex $\Delta_{\left|S_{i}\right|-1}$, Kakutani's theorem (we saw this in Section 2 after Brouwer) ensures this function has a fixed point, which is the desired equilibrium.

Nash gave a second proof using Brouwer's fixed point theorem [301. For this, Nash constructed a continuous function $f$ from the polytope $M$, associated to the game above, into itself. For $m \in M$, we define $f(m)$ componentwise as follows: $f(m)=\left(f_{i}(m), \ldots, f_{N}(m)\right)$, and each entry $f_{i}(m)$ is equal to

$$
\left(f_{i 1}(m), f_{i 2}(m), \ldots, f_{i t_{i}}(m)\right),
$$

where

$$
f_{i j}(m)=\frac{m_{i j}+\max \left(0, u^{i}\left(m_{1}, \ldots, m_{i-1}, s_{j}^{(i)}, m_{i+1}, \ldots, m_{N}\right)-u^{i}\left(m_{1}, \ldots, m_{i}, \ldots, m_{N}\right)\right)}{1+\sum_{k=1}^{\left|S_{i}\right|} \max \left(0, u^{i}\left(m_{1}, \ldots, m_{i-1}, s_{k}^{(i)}, m_{i+1}, \ldots, m_{N}\right)-u^{i}\left(m_{1}, \ldots, m_{i}, \ldots, m_{N}\right)\right)},
$$

where the $s_{j}^{(i)}$ 's are the pure strategies available for player $i$. Nash showed this function $f$ is a multivariate continuous map from the polytope $M$ into itself and thus, by Brouwer's fixed point theorem, it must have at least one fixed point. Nash then went to show that any fixed point of this function is in fact a Nash equilibrium.

The polytope $M=\prod \Delta_{\left|S_{i}\right|-1}$ is actually a Cartesian product of simplices, sometimes called a simplotope. The special structure of simplotopes has been exploited for the computation of fixed points (see [346,374] and references therein) and in the algebraic solution of equilibrium problems via polynomial equations [268. There is one more reason why knowing $M$ is a polyhedron is useful. Despite its geometric beauty and intricacy, there are several modeling limitations with the notion of Nash equilibria. For example, the assumption behind Nash mixed strategies equilibria is that the choices of each player are independent of those of his/her opponents, but that may not hold. Alternative mathematical models that adjust the definition of Nash mixed strategies to allow correlated equilibria appear in 33. In other words, the expression for the payoff function no longer uses the easy product probability structure of a simplotope, but it can have a more complicated polyhedral geometry. For example, it is known that all correlated equilibria are described by a finite set 
of linear inequalities and that it is nonempty, independently of Nash theorem. See [186] and its references.

4.1.2. Two-player games. Nash equilibria have been largely investigated in the area of algorithmic game theory, see for instance the introductory chapter of [304, §2]. We only discuss here some of their relations to combinatorial topology and convexity.

The Nash equilibria for two players can be formulated as a linear complementarity problem, the theory of which subsumes both linear programming and two-player game theory (an introduction is in [116]). Let $A$ and $B$ denote the $m \times n$ payoff matrices of the first and second players, respectively. By definition, a pair $\left(\boldsymbol{x}^{*}, \boldsymbol{y}^{*}\right) \in \Delta_{m-1} \times \Delta_{n-1}$ is a Nash equilibrium if and only if

$$
\boldsymbol{x}^{* T} A \boldsymbol{y}^{*} \geq \boldsymbol{x}^{T} A \boldsymbol{y}^{*} \quad \forall \boldsymbol{x} \in \Delta_{m-1} \quad \text { and } \quad \boldsymbol{x}^{* T} B \boldsymbol{y}^{*} \geq \boldsymbol{x}^{* T} B \boldsymbol{y} \quad \forall \boldsymbol{y} \in \Delta_{n-1}
$$

Here comes the linear complementarity formulation:

Proposition 4.2. The pair $\left(\boldsymbol{x}^{*}, \boldsymbol{y}^{*}\right) \in \Delta_{m-1} \times \Delta_{n-1}$ satisfies (4.1) if and only if there exist $\boldsymbol{u}^{*} \geq \mathbf{0}, \boldsymbol{v}^{*} \geq \mathbf{0}, s \geq 0$, and $t \geq 0$ such that

$$
A \boldsymbol{y}^{*}+\boldsymbol{u}^{*}=s \mathbf{1}, \quad B^{T} \boldsymbol{x}^{*}+\boldsymbol{v}^{*}=t \mathbf{1}, \quad \text { and } \quad \boldsymbol{x}^{* T} \boldsymbol{u}^{*}=\boldsymbol{y}^{* T} \boldsymbol{v}^{*}=0 .
$$

Since all vectors are nonnegative, the conditions on inner products imply that the supports of $\boldsymbol{x}^{*}$ and $\boldsymbol{u}^{*}$ are disjoint, and similarly for $\boldsymbol{y}^{*}$ and $\boldsymbol{v}^{*}$, hence the aforementioned complementarity. The proof of Proposition 4.2 is as follows. Start with a Nash equilibrium $\left(\boldsymbol{x}^{*}, \boldsymbol{y}^{*}\right)$, and let $s=\boldsymbol{x}^{* T} A \boldsymbol{y}^{*}$ and $t=\boldsymbol{x}^{* T} B \boldsymbol{y}^{*}$; the values of $\boldsymbol{u}^{*}$ and $\boldsymbol{v}^{*}$ are forced, and the complementarity conditions follow from multiplying the first equation by $\boldsymbol{x}^{* T}$ and the second equation by $\boldsymbol{y}^{* T}$. Conversely, the complementarity $\boldsymbol{x}^{* T} \boldsymbol{u}^{*}=0$ and $\boldsymbol{x}^{*} \in \Delta_{m-1}$ yield that $s=\boldsymbol{x}^{* T} A \boldsymbol{y}^{*}$ (and similarly $\left.t=\boldsymbol{x}^{* T} B \boldsymbol{y}^{*}\right)$; the positivity of $\boldsymbol{x}^{T} \boldsymbol{u}^{*}$ for any $\boldsymbol{x} \in \Delta_{m-1}$ implies that $\boldsymbol{x}^{*}$ is a best response to $\boldsymbol{y}^{*}$; by a similar argument, $\boldsymbol{y}^{*}$ is a best response to $\boldsymbol{x}^{*}$.

The linear complementarity formulation of Proposition 4.2 yields, after adequate rescaling, the standard method to compute two-player Nash equilibria: find a nontrivial solution (i.e., other than $\boldsymbol{x}=\boldsymbol{y}=\mathbf{0}$ ) to the linear complementarity system

$$
\left(\begin{array}{ll}
A & I_{m}
\end{array}\right)\left(\begin{array}{l}
\boldsymbol{y} \\
\boldsymbol{u}
\end{array}\right)=\mathbf{1}, \quad\left(\begin{array}{ll}
I_{n} & B^{T}
\end{array}\right)\left(\begin{array}{l}
\boldsymbol{v} \\
\boldsymbol{x}
\end{array}\right)=\mathbf{1}, \quad \text { and } \quad \boldsymbol{x}^{T} \boldsymbol{u}=\boldsymbol{y}^{T} \boldsymbol{v}=0
$$

The standard method to solve a linear complementarity system of this form is the Lemke-Howson pivoting algorithm [242] which operates on feasible bases. A feasible basis of a linear system with nonnegativity constraints is a set of indices of columns whose induced submatrix has the same rank as the system and defines a nonnegative solution. The trivial solution $\boldsymbol{x}=\boldsymbol{y}=\mathbf{0}$ gives feasible bases $I_{1}$ for $\left(\begin{array}{c}\boldsymbol{v} \\ \boldsymbol{x}\end{array}\right)$ and $J_{1}$ for $\left(\begin{array}{l}\boldsymbol{y} \\ \boldsymbol{u}\end{array}\right)$, and these bases are complementary, i.e., they have disjoint supports. Pick an (arbitrary) element $k_{1} \notin I_{1}$. By Carathéodory's theorem (in the form of Proposition 3.1),$I_{1} \cup\left\{k_{1}\right\}$ contains another feasible basis $I_{2}$ for the system $B^{T} \boldsymbol{x}+\boldsymbol{v}=1$. Switching from $\left(I_{1}, J_{1}\right)$ to $\left(I_{2}, J_{1}\right)$ is our first pivot step. We remark that $I_{2}$ and $J_{1}$ are no longer disjoint, as they share $k_{1}$. To remedy this, note that the part of $I_{2}$ corresponding to $\boldsymbol{v}$ lost some element $k_{2}$ which is also absent from the part of $J_{1}$ corresponding to $\boldsymbol{y}$. We can thus make $k_{2}$ enter $J_{1}$ without further degrading the complementarity; Carathéodory's theorem ensures that $J_{1} \cup\left\{k_{2}\right\}$ contains another feasible basis $J_{2}$, and we pivot from $\left(I_{2}, J_{1}\right)$ to $\left(I_{2}, J_{2}\right)$. The part 
of $J_{2}$ corresponding to $\boldsymbol{u}$ lost some element $k_{3}$. If $k_{3}=k_{1}$, then $I_{2}$ and $J_{2}$ are complementary, and they determine our nontrivial solution; otherwise, we continue pivoting by making $k_{3}$ enter $I_{2}$, etc., until a pivot makes $k_{1}$ exit one of the bases. The pair at hand is then complementary.

The Lemke-Howson algorithm is guaranteed to terminate under the nondegeneracy assumption that $\mathbf{1}$ is not a positive combination of less than $m$ columns of ( $\left.\begin{array}{ll}A & I_{m}\end{array}\right)$ or less than $n$ columns of $\left(\begin{array}{ll}I_{n} & B^{T}\end{array}\right)$. This follows from a nondegenerate Carathéodory theorem (after Proposition 3.1): any point in the convex hull of $d+2$ points of $\mathbb{R}^{d}$ that is nondegenerate (i.e., is not in the convex hull of some $d$ of them) lies in the convex hulls of exactly two $(d+1)$-element subsets. (Note that the two nondegeneracy assumptions stated above are equivalent via the convex/conic change of viewpoint.) Now, for any pair $(I, J)$ of noncomplementary feasible bases encountered by the algorithm, there is exactly one index $k$ not in $I \cup J$. A pivot step makes $k$ enter either $I$ or $J$; in each case, the nondegenerate Carathéodory theorem yields exactly one other pair of feasible bases. Every pair $(I, J)$ of noncomplementary feasible bases encountered by the algorithm thus has exactly two neighbors through pivot steps. Since the trivial solution $\left(I_{1}, J_{1}\right)$ has exactly one neighbor, there is no place where the walk can loop back. We remark that this algorithm gives an alternate (constructive) proof of the existence of a Nash equilibrium for two-player games.

The argument that proves termination also reveals that, from a computational complexity point of view, linear complementarity systems of the form of equation (4.2) are in the PPAD class. Let us point out that the Lemke-Howson algorithm can be understood as a Sperner-type search for a fully labeled simplex in a pseudomanifold. Let $V=\{ \pm 1, \pm 2, \ldots, \pm(m+n)\}$, where positive integers are understood as indices of columns of $\left(\begin{array}{lll}A & I_{m}\end{array}\right)$ and negative integers are understood as (minus) indices of columns of $\left(I_{n} \quad B^{T}\right)$. Consider the simplicial complex $\mathrm{K}$ on $V$ whose maximal simplices are the union of the complement of a feasible basis of ( $\left.\begin{array}{ll}A & I_{m}\end{array}\right)$ and the complement of a feasible basis of $\left(\begin{array}{lll}I_{n} & B^{T}\end{array}\right)$. The nondegenerate Carathéodory theorem spelled out above ensures that $\mathrm{K}$ is a pseudomanifold without boundary. If every $i \in V$ is labeled by its absolute value $|i|$, the fully labeled simplices correspond exactly to the complementary feasible bases.

As a byproduct of the linear complementarity formulation of Proposition 4.2 . we also get that the problem of computing a Nash equilibrium for two players is well-posed from the point of view of computational complexity: if the input involves only rational data, there is an equilibrium that involves only rational data and has an encoding size polynomial in the input size (see for instance the discussion in the survey of McKelvey and McLennan [267]). This is in sharp contrast with the case of three or more players: Nash's three-player poker game [301] shows that a three-player game with finitely many strategies and rational payoff arrays may have a (unique) Nash equilibrium with irrational coordinates.

The Lemke-Howson algorithm has exponential complexity in the worst case [337, and solving general linear complementarity problems is NP-hard [107. The problem of computing a Nash equilibrium for two players is PPAD-complete [104] (see also [121]). The intractability for games with three or more players is even more stringent, as many decision problems are $\exists \mathbb{R}$-complete, i.e., as difficult as deciding the emptiness of a general semi-algebraic set; in particular this includes deciding whether a three-player game has a Nash equilibrium within $\ell_{\infty}$-distance $r$ 
from a given distribution $\boldsymbol{x}$ [341, Corollary 5.5] or the existence of more than one equilibrium or of equilibria with payoff or support conditions [181. Behind this $\exists \mathbb{R}$-completeness lurks a more daunting fact: Datta's universality theorem [122] asserts that arbitrarily complicated semi-algebraic sets can be encoded as sets of Nash equilibria (formally: every real algebraic variety is isomorphic to the set of mixed Nash equilibria of some multiplayer game). Whether the $\exists \mathbb{R}$-completeness results stated above could follow from Datta's proof is an interesting open question [341, Remark 5.6].

Open Problem 4.3. Can Datta's universality theorem be improved to yield an efficient polynomial-time reduction between any semi-algebraic set and the Nash equilibria of a game?

Another open question is whether both few players and few strategies per player already give rise to universality.

Open Problem 4.4. Is there a universality result for the set of Nash equilibria of games with a constant number of players and a constant number of strategies?

4.1.3. Zero-sum games. The two-player games where what is won by a player is lost by the other are called zero-sum games; this is the case when the payoff matrices satisfy $A=-B$ in the formulation (4.1). In this special case, it is customary to consider that one player aims at maximizing the payoff while the other tries to minimize it. Nash's theorem then asserts that there exist $\boldsymbol{x}^{*} \in \Delta_{n-1}$ and $\boldsymbol{y}^{*} \in \Delta_{m-1}$ such that

$$
\begin{aligned}
& \forall \boldsymbol{y} \in \Delta_{m-1}, \quad \boldsymbol{x}^{* T} A \boldsymbol{y} \geq \boldsymbol{x}^{* T} A \boldsymbol{y}^{*}, \\
& \forall \boldsymbol{x} \in \Delta_{n-1}, \quad \boldsymbol{x}^{T} A \boldsymbol{y}^{*} \leq \boldsymbol{x}^{* T} A \boldsymbol{y}^{*}
\end{aligned}
$$

This readily implies

$$
\begin{aligned}
\boldsymbol{x}^{* T} A \boldsymbol{y}^{*} & \geq \max _{\boldsymbol{x} \in \Delta_{n-1}} \boldsymbol{x}^{T} A \boldsymbol{y}^{*} \geq \min _{\boldsymbol{y} \in \Delta_{m-1}} \max _{\boldsymbol{x} \in \Delta_{n-1}} \boldsymbol{x}^{T} A \boldsymbol{y} \\
& \geq \max _{\boldsymbol{x} \in \Delta_{n-1}} \min _{\boldsymbol{y} \in \Delta_{m-1}} \boldsymbol{x}^{T} A \boldsymbol{y} \geq \min _{\boldsymbol{y} \in \Delta_{m-1}} \boldsymbol{x}^{* T} A \boldsymbol{y} \geq \boldsymbol{x}^{* T} A \boldsymbol{y}^{*} .
\end{aligned}
$$

The only inequality that does not follow from equation (4.3), the central one, holds in fact for arbitrary bivariate functions (see Section 6.4.2). Altogether, this yields the min-max theorem of von Neumann.

Theorem 4.5. For any $A \in \mathbb{R}^{m \times n}$,

$$
\max _{\boldsymbol{x} \in \Delta_{n-1}} \min _{\boldsymbol{y} \in \Delta_{m-1}} \boldsymbol{x}^{T} A \boldsymbol{y}=\min _{\boldsymbol{y} \in \Delta_{m-1}} \max _{\boldsymbol{x} \in \Delta_{n-1}} \boldsymbol{x}^{T} A \boldsymbol{y}
$$

In game-theoretic language, the real number $\boldsymbol{x}^{* T} A \boldsymbol{y}^{*}$ is the value of the game. Von Neumann's theorem has a nice asynchronous interpretation: for any choice of a strategy by the minimizing player, the maximizing player can respond so as to ensure a payoff at least the value of the game. Moreover, if the maximizing player cares only about achieving the value of the game, the strategy $\boldsymbol{x}^{*}$ will work regardless of what the opponent plays. (Of course, symmetric statements hold for the minimizing player.) In zero-sum games, in each of the (possibly many) Nash equilibria, every player gets the same payoff. This is specific to zero-sum games and fails already for broader types of two-player games. A classical result of Dantzig [119 says that the minmax identity of von Neumann's theorem can be proved, without help of combinatorial topology, via linear programming duality and is thus 
polynomially solvable. We will discuss more about this in Section 6. More generally, if the rank of $A+B$ is constant, then the problem is polynomial [223].

Formulation (4.1) suggests an approximate relaxation and the approximate Carathéodory theorem, Theorem 3.5 provides a positive complexity result. We say a mixed strategy pair $(\boldsymbol{x}, \boldsymbol{y})$ is an $\varepsilon$-Nash equilibrium if

$$
\boldsymbol{x}^{T} A \boldsymbol{y} \geq \boldsymbol{e}_{i}^{T} A \boldsymbol{y}-\varepsilon \quad \forall i \in[n] \quad \text { and } \quad \boldsymbol{x}^{T} B \boldsymbol{y} \geq \boldsymbol{x}^{T} B \boldsymbol{e}_{j}-\varepsilon \quad \forall j \in[n] .
$$

Intuitively, a mixed strategy pair is an $\varepsilon$-Nash equilibrium if no player can benefit more than $\varepsilon$, in expectation, by a unilateral deviation.

The case when $A+B=0$ is precisely the case of zero-sum games, for which we know efficient algorithms exist. Barman, using the approximate Carathéodory theorem presented in Theorem 3.5. provided an extension in [51].

Theorem 4.6. Suppose that all entries of the payoff matrices $A, B$ lie in $[-1,1]$. If the number of nonzero entries in $A+B$ is at most $s$, then an $\varepsilon-N a s h$ equilibrium of $(A, B)$ can be computed in time $n^{O\left(\frac{\log (\max (s, 4))}{\varepsilon^{2}}\right)}$.

This, in particular, gives a polynomial-time approximation scheme for Nash equilibrium in games with fixed column sparsity $s$. Moreover, for arbitrary bimatrix games - since $s$ can be at most $n$ - the running time of this algorithm matches the best-known upper bound, which was obtained by Lipton, Markakis, and Mehta [243.

4.2. Two fair-division problems: cakes and necklaces. In various situations players are eager to divide goods in a fair way. There are several examples of such fair-division problems where our five theorems (or their variations) play a key role. We review some famous examples, all with combinatorial-topological proofs. Before we start, we remark there are other interesting mathematical challenges arising in distributing resources, some we will not cover here, such as gerrymandering, which is the practice of drawing political maps to gain an advantage; see, e.g., 356 for connections to the five theorems in this survey.

Cake cutting. A cake is a metaphor for a heterogeneous, divisible good, such as a piece of land or an inheritance. We consider the problem of dividing a cake between $r$ players in such a way that each player prefers his or her part to any other part. We call this envy-free. Let us point out that the literature about fair division, including this and other types of cake-cutting problems, is both old and huge; see e.g., [77 79, 324, 328, 363. For example, one of the first envy-free division results was shown by Dubins and Spanier [146].

One setting where pieces are connected is the following: The cake is identified with the unit interval $[0,1]$ and a division of the cake into $r$ pieces is an $r$-tuple $\boldsymbol{x}=\left(x_{1}, \ldots, x_{r}\right)$, with $x_{j} \geq 0$ for all $j \in[r]$ and $\sum_{j=1}^{r} x_{j}=1$; in other words, a division is a point $\boldsymbol{x}$ in the $(r-1)$-dimensional simplex $\Delta_{r-1}$. Here, $x_{j}$ represents the size of the $j$ th piece, when ordered from left to right. The preferences of player $i$ are modeled by a function $P^{i}$ mapping each division $\boldsymbol{x} \in \Delta_{r-1}$ to a nonempty subset of $[r]$ (indexing the pieces that he or she prefers). A division $\boldsymbol{x}$ is envy-free if there exists a choice of pairwise distinct indices, one from each $P^{i}(\boldsymbol{x})$.

It is natural to assume that the set $P^{i}(\boldsymbol{x})$ of preferences of player $i$ never contains the index of a piece of size zero (i.e., all players are hungry), and it is common to suppose that the $P^{i}$ 's are closed, that is if $\lim _{n \rightarrow \infty} \boldsymbol{x}_{n}=\boldsymbol{x}$ and $j \in P^{i}\left(\boldsymbol{x}_{n}\right)$ for every 
$n$, then $j \in P^{i}(\boldsymbol{x})$. Stromquist [366] and Woodall [392] proved the following result independently.

Theorem 4.7. Under the assumptions that all $r$ players are hungry and the preference functions $P^{i}$ are closed, there exists an envy-free division with connected pieces.

The original proof relies on the KKM theorem we saw in Section 2, An unpublished proof due to Forest Simmons was improved and adapted by $\mathrm{Su}$ 368. The idea is to refine the usual derivation of Brouwer's theorem from Sperner's lemma. Take a sequence $\left(T_{n}\right)$ of triangulations of $\Delta_{r-1}$ whose edge-length goes to 0. Assign every vertex to a player in a way that every full-dimensional simplex of $\mathrm{T}_{n}$ has a vertex assigned to each player; this may not be possible for any sequence of triangulations, but taking iterated barycentric subdivisions does the job. We label or color every vertex $\boldsymbol{x}$ assigned to player $i$ by some (arbitrary) element from $P^{i}(\boldsymbol{x})$. The assumption that players are hungry ensures that this is a Sperner labeling. The limit of a converging subsequence of fully labeled simplices is, by the closedness assumption, an envy-free division.

It may be disappointing in practice that one only gets an iterative infinite process converging to an envy-free division, but it has been shown in 367. that there exists no finite procedure, if you require each person to get a connected piece (i.e., the cake is cut by a minimal number of cuts). In fact, Aziz and Mackenzie [38] showed that there is in fact a bounded finite procedure for $r$-person cake cutting bounded by a huge number of steps, but this would involve breaking the cake into a ridiculous number of pieces. Thus for now one cannot get a minimal number of cuts in a finite procedure, but if you allow a lot of cuts, you get a division that is impractical since it destroys the cake. This is why an infinite process converging to an envy-free solution with a minimal number of cuts makes sense. Let us comment that the difficulty of the process is not so surprising perhaps. It is known that in the polynomial-time function model, where the utility functions are given explicitly by polynomial-time algorithms, the envy-free cake-cutting problem has the same complexity as finding a Brouwer's fixed point, or, more formally, it is PPAD-complete 138.

The polytopal version of Sperner's lemma (Proposition 2.5] in Section 2.3) has many interesting game-theoretic applications. Su 369 recently gave a simple, elegant proof that Hex does not end in a draw when using it. Cloutier, Nyman, and $\mathrm{Su}$ [114 applied the polytopal Sperner's lemma to multi-cake multi-player fair-division problems. In this type of problem the players have several cakes to choose from, but choices from one cake influence each other, e.g., for a player the amount of vanilla cake may influence how much chocolate cake to order, or after some vanilla cake the player may not want any chocolate cake. Cloutier et al. asked whether there exists an integer $r(q, m)$, independent of the preferences, such that there exists an envy-free division of the $m$ cakes not requiring the division of each cake into more than $r(q, m)$ pieces, some of which are assigned to each of the $q$ players (some of the pieces can remain unassigned). Note that Theorem 4.7 for a single cake asserts that $r(q, 1)=q$. They also used the polytopal version of Sperner's lemma, and they proved the existence of $r(2,2)$ and $r(2,3)$ and that $r(2,2)=3$ and $r(2,3) \leq 4$. This means that two cakes can be divided into three pieces each in such a way that two players receive the pieces and everyone is satisfied with the fairness of division. Moreover they asked whether $r(2, m) \leq m+1$. Recently, Lebert, Meunier, and Carbonneaux 241] have shown $r(2, m)$ exists for any $m \geq 2$ and its value is at 
most $m(m-1)+1$. Again they used the polytopal version of Sperner's lemma and an inequality between the matching number and the fractional matching number in $m$-partite hypergraphs. Similarly, they showed $r(3,2)$ exists and $r(3,2) \leq 5$. Several interesting open questions remain; consider the following.

Open Problem 4.8. Can the bound on $r(2, m)$ be improved? Can one assure the existence of $r(q, m)$ for all values of $q, m$ ?

Finally, there are other surprising variations of the cake-division theorem. Consider one where there are no cuts involved and one divides objects that are not physically divisible. Consider a house with $n$ rooms and a total rent amount to be divided among $n$ roommates. Assume that for each possible division of the rent amount each roommate can point to one or more rooms as preferred. Then, the theorem proved by $\mathrm{Su}$ in 368 states that there exists a division of the rent and an assignment of rooms to each participant, such that each player receives one of his/her preferred rooms. Similar assumptions on the preference function that we made for cake-cutting before Theorem 4.7 must hold again to make this happen; for instance, now it is assumed that each roommate prefers a free room over paying rent. Once more the proof of this theorem is grounded in Sperner's lemma. In most results on fair division it is assumed that no player is happy with an empty piece of cake, but imagine a part of the cake is undesirable (burnt cake anyone?) another recent variation in 278, 348, considers the possibility players may prefer an empty piece.

It is well-known that Gale's colorful KKM theorem (see Section 2.2) has interesting applications in economics, e.g., for the existence of economics equilibria. Now, Asada et al. 32 used Gale's colorful KKM theorem to prove an extension, by Woodall [392, of Theorem 4.7] and a similar extension of the rental-harmony result of $\mathrm{Su}$. It turns out that there are envy-free cake divisions for any number of players, even if the preferences of one person remain secret! Say the situation is one where one of the cake-cutters (maybe the person celebrating a birthday) is not providing preference information, but still the cutting of the cake can be made without anyone being envious. Similarly, for deciding what the rent should be, it suffices to consider the information of all but one of the roommates and still none of them will be jealous. The authors provided a rather nice existence proof of such fair divisions. Recently Frick, Houston-Edwards, and Meunier gave an iterative approximation algorithm for the solution [171.

Necklace splitting. Another fair division problem asks for fair splitting, between two thieves, of an open necklace with beads threaded along the string. Here, fair means that for each type of bead the number of beads assigned to each of the thieves differs by at most one (say because the thieves are unaware of the value of the beads). Perhaps surprisingly, this can be achieved using only a few cuts (which turns out to be convenient should the string material be precious). Contrary to the classical statement, with this notion of fairness we do not need to add any conditions on divisibility.

Theorem 4.9 (Necklace theorem). There exists a fair splitting of a necklace that uses no more cuts than there are bead types.

The result is optimal because some bead arrangements need $k$ cuts, where $k$ equals the number of bead types (e.g., such a situation happens when all beads 
of the same type are consecutive). Theorem 4.9 was first proved by Goldberg and West [190, and a simpler proof using the Borsuk-Ulam theorem was proposed by Alon and West [24], who also came up with the above popular formulation. More generally, the challenge of finding a division of an object into two portions so that each of $n$ people believes the portions are equal is called consensus-halving. Note that necklace splitting is a special case because different preferences are represented by different beads. In 353. Simmons and Su showed how a nonconstructive existence result on consensus-halving can be obtained from the Borsuk-Ulam theorem. They also showed, by a direct application of Tucker's lemma, how one can construct an approximate consensus-halving (up to a prespecified tolerance level).

Later on, a combinatorial proof due to Pálvölgyi [313] used the octahedral Tucker lemma, instead of the Borsuk-Ulam theorem, for necklace-splitting. Here is a sketch of that idea. Let $n$ and $t$ denote the numbers of beads and bead types, respectively, let $a_{j}$ denote the number of beads of type $j$, and assume, ad absurdum, that any fair splitting uses more than $t$ cuts. Every vector $\boldsymbol{x} \in\{+,-, 0\}^{n}$ defines a partial assignment of the beads to the two thieves (identified with + and - , respectively). If every extension of $\boldsymbol{x}$ into a complete assignment has at most $t$ cuts, then no such extension can be a fair splitting, and there is some $j$ such that more than $\frac{a_{j}}{2}$ beads of type $j$ are assigned to the same thief by $\boldsymbol{x}$. Define $\lambda(\boldsymbol{x})$ to be the smallest such index $j$, signed by the thief who gets more than $\frac{a_{j}}{2}$ beads of that type. If there is an extension of $\boldsymbol{x}$ into a complete assignment with more than $t$ cuts, define $\lambda(\boldsymbol{x})$ to be the maximum number of cuts achieved by a completion, signed by the first component of that completion (that this sign is well-defined is straightforward). This map $\lambda$ satisfies the condition of the octahedral Tucker lemma with $m=n-1$ and therefore cannot exist, contradicting the initial assumption.

The necklace splitting problem naturally generalizes from 2 to any number $q$ of thieves. Alon [19] showed that $(q-1) t$ cuts suffice (note that they are sometimes necessary). His proof first assumes $q$ to be prime and replaces (in a nontrivial way) the stronger form of the Borsuk-Ulam theorem due to Bárány, Shlosman, and Szücs (see [19, Lemma 4.1] or [48, Statement $\left.\mathrm{B}^{\prime}\right]$ ). The case of general $q$ follows easily by a recursive argument. (The original proof assumed $a_{j}$ to be divisible by $q$ but this was subsequently relaxed by Alon et al. 23, who obtained fair roundings via integrality properties of flows.) It is not known, however, whether Pálvölgyi's proof can be adapted to the case with more than two thieves; perhaps an ingredient for this could be $\mathbb{Z}_{q}$-generalizations of the octahedral Tucker lemma [274,395]. Another open question [313] relates to the rounding: For those $a_{j}$ 's not divisible by $q$, can one choose the thieves who get the additional beads in the fair splitting? This is easily seen to be true for two thieves, and it is also true for three [18]; it is open for $q \geq 4$.

Open Problem 4.10. Is it possible to choose for each type $j$ the thieves who get $\left\lceil a_{j} / q\right\rceil$ and those who get $\left\lfloor a_{j} / q\right\rfloor$ in the fair splitting?

For two thieves, a fair splitting can be computed in linear time for $t=2$ and in $O\left(n^{t-2}\right)$ time for $t \geq 3$ [190]. Let us mention again consensus-halving (the problem of dividing an object into two portions so that each of $n$ players believes the portions are equal) appears in many contexts. In 353] the Borsuk-Ulam theorem and Tucker's lemma were used for this purpose. A well-known challenge of computational complexity was to decide whether the computation of a fair splitting is a PPA-complete problem 314]. This was just recently settled by Filos-Ratsikas and 
Goldberg 163, 164. The fact that necklace splitting is PPA-complete implies that the algorithmic version of the octahedral Tucker lemma is PPA-complete as well, but there is also a paper directly proving that the algorithmic octahedral Tucker's lemma is PPA-complete 137. (see Section 2.4).

The topic of fair-division is very active, and once more we can only point to a few additional types of results. One may, for instance, explore necklace splittings with the added constraint that adjacent pieces of the necklace cannot be claimed by certain pairs of thieves; for example, Asada et al. 32] prove that four thieves on a circle can share the beads of the necklace, with the restriction that the two pairs of nonadjacent thieves will not receive adjacent pieces of the necklace. There are also several nice high-dimensional generalizations of (convex) splitting of booty; see [70,134 and the references therein.

\section{GRAPHS}

Graphs are often used to model problems where pairwise interactions are prominent. This includes situations where graph-like structures are apparent, for instance road or train networks, or situations as in Euler's famous problem on the bridges of Königsberg (although the curious reader may check that Euler's original article does not use any graph-related notions, but argues purely in terms of words coding paths). In other situations, graphs are not evident, but they exist implicitly; for instance, describing time dependencies between tasks in scheduling problems.

Graph theory developed in many independent directions, driven both by applications (e.g., finding graph matchings to resolve assignments [345]) and deep structural questions (e.g., the graph minor theory [248]). Its interaction with (combinatorial) topology started in the mid-1970s with the proof by Lovász [62, Theorem 6.1] of the conjecture of Frank and Maurer that any $k$-connected graph $G=(V, E)$ can be partitioned into $k$ subsets that induce connected subgraphs and have prescribed size (summing to $|V|$ ), and in which each contains a prescribed element. (This result was independently given a nontopological proof by Győry [199].) Lovász [247] followed up shortly after with an astonishing solution to the Kneser conjecture based on the Borsuk-Ulam theorem.

5.1. Chromatic number of graphs. A coloring of a graph by $k$ colors is a map from its vertex set into $[k]$; a coloring is proper if any adjacent vertices have different colors. The chromatic number of a graph is the smallest integer $k$ such that a proper coloring by $k$ colors exists. Graph colorings arise in applications such as frequency assignment [1] or scheduling [136].

Proving good lower bounds on chromatic numbers is usually a difficult task, as one needs to show that all colorings with fewer colors are improper. (In contrast, proving an upper bound on the chromatic number of a given graph only requires that one proper coloring be exhibited.) In some cases, such as the perfect graphs discussed in Section 5.3. sharp lower bounds can be obtained from the existence of large cliques. Kneser graphs are archetypes where this clique criterion fails dramatically. The Kneser graph $\operatorname{KG}(n, k)$, where $n$ and $k$ are two integers, is the graph with vertex set $\left(\begin{array}{c}{[n]} \\ k\end{array}\right)$ - the $k$-element subsets of $[n]$ - and where two subsets form an edge if they are disjoint. When $n \geq 2 k-1$, a natural coloring of $\operatorname{KG}(n, k)$ assigns to every $k$-element subset that intersects $[n-2 k+1]$ its minimal element and $n-2 k+2$ to all remaining subsets (see Figure 12, and note that $\operatorname{KG}(5,2)$ is also known as Petersen's graph). Kneser conjectured this to be optimal in 1955. 


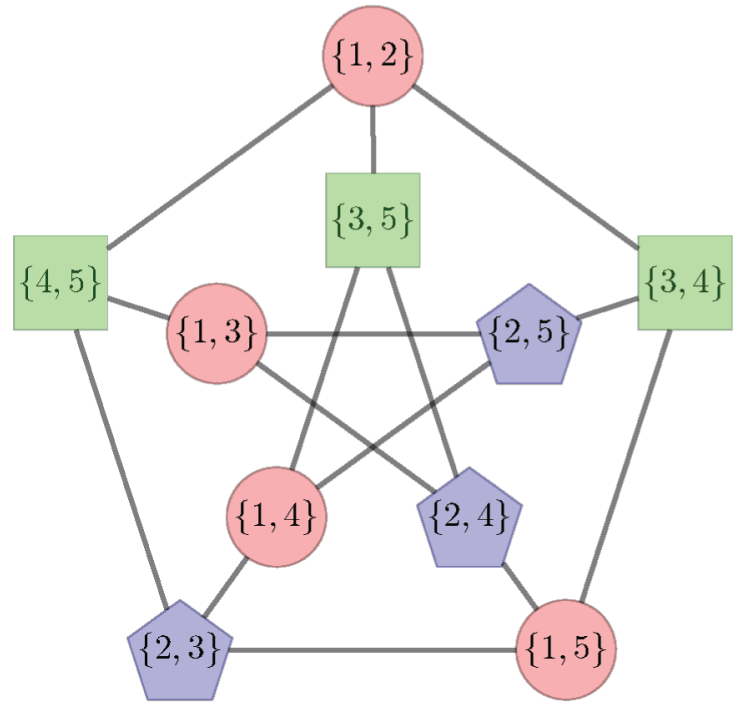

Figure 12. A proper coloring with three colors: 1-red, 2-blue, 3 -green on the Kneser graph $\mathrm{KG}(5,2)$.

Lovász approached the Kneser conjecture via a topological invariant. Given a graph $G=(V, E)$, consider the simplicial complex $N(G)$ that encodes its neighborhoods: $N(G)$ has the same vertex set as $G$, and a subset of the vertices forms a simplex whenever they have a common neighbor in $G$. The key invariant is the (homotopy) connectivity of $N(G)$ : if $N(G)$ is $(k-1)$-connected, then $G$ is not $(k+1)$-colorable. In the case of Kneser graphs, this yields a lower bound that proves the conjecture. The interested reader may refer to the book of Matoušek [258, Section 3.3] and the surveys of Björner [62, Section 6] and Bárány [41, Section 5] for details.

The idea of associating a simplicial complex to a graph and to relate the chromatic number of the latter to topological properties of the former has been especially fruitful, and there are now various complexes that can be used to obtain lower bounds for the chromatic number of a graph; see [263] and [234, Chapter 19] for surveys on that approach. Recently, Frick used Tverberg-type results to show bounds for chromatic numbers of (generalized) Kneser graphs and hypergraphs [169, 170.

The octahedral Tucker lemma emerged from a purely combinatorial proof of the Kneser conjecture due to Matoušek 259]. Ziegler 395] then showed that his method can combinatorialize other topological arguments for chromatic numbers. Let us illustrate this method on a bound due to Dol'nikov which deals with hypergraphs.

Recall that a hypergraph is a pair $\mathcal{H}=(V, E)$ where $V$ is a finite set (the vertices) and $E$ is a set of subsets of $V$ (the edges); in particular, hypergraphs whose edges all have size 2 are graphs. Given a hypergraph $\mathcal{H}=(V, E)$ and a subset $S \subseteq V$ of its vertices, the hypergraph $\mathcal{H}[S]$ induced on $S$ has vertex set $S$ and edge set $\{e \in E: e \subseteq S\}$. A hypergraph $\mathcal{H}$ is 2-colorable if $V$ can be colored so that no edge is monochromatic. To any hypergraph $\mathcal{H}=(V, E)$, we associate the generalized Kneser graph $\operatorname{KG}(\mathcal{H})=\left(V^{\prime}, E^{\prime}\right)$ where

$$
V^{\prime}=E \quad \text { and } \quad E^{\prime}=\{e f: e, f \in E \text { and } e \cap f=\varnothing\} .
$$


In particular, the generalized Kneser graph of the $k$-uniform hypergraph with vertex set $[n]$ is the usual Kneser graph $\operatorname{KG}(n, k)$. The colorability defect $\operatorname{cd}(\mathcal{H})$ of a hypergraph $\mathcal{H}$ is the minimum number of vertices to be removed from $\mathcal{H}$ to ensure that the remaining vertices can be 2 -colored so that no edge of $\mathcal{H}$ is monochromatic (edges with at least one removed vertex are discarded).

Theorem 5.1 (Dol'nikov [145]). For any hypergraph $\mathcal{H}$, the chromatic number of $\operatorname{KG}(\mathcal{H})$ is at least $\operatorname{cd}(\mathcal{H})$.

The combinatorial proof of Theorem 5.1 goes essentially as follows. Consider a hypergraph $\mathcal{H}$ with vertex set (identified with) $[n]$. Given a proper coloring $c$ of $\operatorname{KG}(\mathcal{H})$ by $[t]$, we define a map $\lambda:\{+,-, 0\}^{n} \backslash\{\mathbf{0}\} \rightarrow\{ \pm 1, \ldots, \pm m\}$ as follows. Consider $\boldsymbol{x} \in\{+,-, 0\}^{n}$. If at least one edge of $\mathcal{H}$ is entirely contained in $\boldsymbol{x}^{+}$or in $\boldsymbol{x}^{-}$, then we choose such an edge with smallest color $a$, and we set

$$
\lambda(\boldsymbol{x})=\varepsilon(n-\operatorname{cd}(\mathcal{H})+a),
$$

where $\varepsilon \in\{-,+\}$ records which of $\boldsymbol{x}^{+}$or $\boldsymbol{x}^{-}$contains the edge. (The sign $\varepsilon$ is unambiguously defined because the coloring is proper.) If neither $\boldsymbol{x}^{+}$nor $\boldsymbol{x}^{-}$ contains an edge of $\mathcal{H}$, then we define $\lambda(\boldsymbol{x})$ to be $\varepsilon\left(\left|\boldsymbol{x}^{+}\right|+\left|\boldsymbol{x}^{-}\right|\right)$, where the sign $\varepsilon$ is the first nonzero entry of $\boldsymbol{x}$. In the latter case, the edges of $\mathcal{H}$ contained in $\boldsymbol{x}^{+} \cup \boldsymbol{x}^{-}$ induce a sub-hypergraph of $\mathcal{H}$ that is 2-colorable, so $\left|\boldsymbol{x}^{+} \cup \boldsymbol{x}^{-}\right| \leq n-\operatorname{cd}(\mathcal{H})$. It then follows that $m=n-\operatorname{cd}(\mathcal{H})+t$ suffices and that in either case, $\boldsymbol{x}$ is mapped to disjoint sets of labels, which helps in checking that $\lambda$ satisfies the condition of the octahedral Tucker lemma. As a consequence, $n-\operatorname{cd}(\mathcal{H})+t \geq n$, and the announced inequality follows.

Since every graph is isomorphic to some (actually, many) generalized Kneser graph, Theorem 5.1 provides a lower bound on the chromatic number of any graph. In the case of Kneser graphs, this bound is sharp. There exist refinements of the colorability defect that yield better combinatorial bounds [16]. Cases of equality for Theorem 5.1 are remarkable for reasons related to circular chromatic numbers [17] (see 394] for an introduction to circular chromatic numbers). Deciding whether the chromatic number of $\operatorname{KG}(\mathcal{H})$ equals $\operatorname{cd}(\mathcal{H})$ is a natural problem asked in [17. Very recently, this has been proved to be NP-hard by Meunier and Mizrahi (personal communication).

Some generalizations of the Kneser conjecture are still open. A $k$-element subset $A$ of $[n]$ is $s$-stable if for any $i, j \in A$ we have $s \leq|i-j| \leq n-s$ (or, equivalently, if $i$ and $j$ are distance at least $s$ apart in $\left.\mathbb{Z}_{n}\right)$. Let $\mathrm{KG}_{s \text {-stab }}(n, k)$ denote the graph with vertices the $s$-stable $k$-element subsets of $[n]$, and where two vertices span an edge if they are disjoint. The graph $\mathrm{KG}_{2 \text {-stab }}(n, k)$, known as Schrijver's graph [343, has the same chromatic number as the Kneser graph; since $\mathrm{KG}_{2 \text {-stab }}(n, k)$ is a subgraph of $\mathrm{KG}(n, k)$, this strengthens Lovász's result. As a special case of a conjecture on hypergraphs, Meunier 274 proposed the following.

Conjecture 5.2. For any $s \geq 2$ and $n \geq k s \geq 1$, the chromatic number of $\mathrm{KG}_{s \text {-stab }}(n, k)$ is $n-s(k-1)$.

Besides the case $s=2$, the conjecture is known for all even $s$ [100] and for $s \geq 4$ and $n$ sufficiently large [216]. Some progress has been made by Chen [102; see also [170] for questions related to Kneser hypergraphs.

A more systematic viewpoint recasts graph colorings as special cases of graph homomorphisms [200]. A homomorphism from a graph $G=(V, E)$ to a graph 
$H=(W, F)$ is a map $f: V \rightarrow W$ such that for every edge $v v^{\prime} \in E$, the image $f(v) f\left(v^{\prime}\right)$ is an edge of $H$, that is $f(v) f\left(v^{\prime}\right) \in F$. A proper coloring of $G$ with $k$ colors corresponds to a homomorphism from $G$ to the complete graph with $k$ vertices. More generally, associating with every vertex of $G$ a $k$-element subset of $[n]$ so that adjacent vertices have disjoint subsets amounts to finding a homomorphism from $G$ to $\operatorname{KG}(n, k)$. The structure of homomorphisms of Kneser graphs remains to be elucidated, as is shown by the following, broadly open, conjecture.

Conjecture 5.3 (Stahl [360]). Let $n, k, k^{\prime}$ be integers, and let $q$ and $r$ be such that $k^{\prime}=q k-r$ with $0 \leq r<k$. There is a homomorphism $\mathrm{KG}(n, k) \rightarrow \mathrm{KG}\left(n^{\prime}, k^{\prime}\right)$ if and only if $n^{\prime} \geq q n-2 r$.

For more details on partial progress on Stahl's conjecture, see [200, §3.4].

5.2. Colorful independent sets. A subset of vertices of a graph is independent if there is no edge between any pair of them. Independent sets are sometimes called stable sets. This notion is central in graph theory. For instance, a proper coloring of a graph can be understood as a partition of its vertices into independent sets, namely the sets of vertices with the same color. The search for independent sets is also natural in applications such as the design of error-correcting codes [261, §29].

Methods from combinatorial topology were particularly effective in finding independent sets with color constraints in the spirit of the colorful theorems in combinatorial convexity. The following example was first stated explicitly by Aharoni et al. [7, who traced it back to the proof of a result of Haxell [204, Theorem 3] on hypergraph matchings.

Theorem 5.4. Let $G$ be a colored graph with maximum degree $\Delta$. There exists an independent set of $G$ that intersects every color class of size at least $2 \Delta$.

(Note that the coloring of $G$ is not required to be proper.) It suffices to prove the statement in the case where every color class has size at least $2 \Delta$ because deleting every vertex in a color class of size less than $2 \Delta$ preserves independence and does not augment the maximum degree. The gist of the proof of Aharoni et al. [7] is to apply Meshulam's lemma (Proposition [2.6) to the independence complex $\mathrm{K}$ of $G$, the simplicial complex consisting of its independent sets. The connectivity of $\mathrm{K}$ can be controlled via a variety of domination graph parameters; this principle, which underlies some proofs of Meshulam [271, was made explicit by Aharoni et al. [7, Theorem 2.3] and given a detailed proof by Adamaszek and Barmak [2]. In particular, given a coloring of $V(G)$ by $[k]$, a subset $I \subseteq[k]$, and an integer $j$, if no $2 j+3$ vertices of $G$ dominate the vertices with colors in $I$, then $\widetilde{H}_{j}\left(\mathrm{~K}\left[\lambda^{-1}(I)\right], \mathbb{Z}_{2}\right)$ is trivial. (Recall that $X$ dominates $Y$ if every vertex of $Y$ has a neighbor in $X$.) Here, the condition holds for $j=|I|-2$ because dominating $2 \Delta|I|$ vertices requires at least $2|I|$ vertices when the maximum degree is $\Delta$. By Meshulam's lemma (Proposition 2.6), therefore, the independence complex contains a colorful simplex. This is an independent set that intersects every color class.

Theorem 5.4 can be improved for graphs with more structure as the following example shows [18].

Theorem 5.5. In any coloring of a path, it is possible to delete a vertex of each color so that the remaining vertices can be partitioned into two independent sets $A$ and $B$ such that $-1 \leq|A \cap U|-|B \cap U| \leq 1$ for every color class $U$. 
(Again, the coloring does not need to be proper.) We sketch here a direct proof based on the octahedral Tucker lemma in a way reminiscent of Pálvolgyi's proof of the necklace theorem (Theorem 4.9). We identify the vertex set of the path with $[n]$ and denote the color classes by $U_{1}, \ldots, U_{t}$. The existence of the two disjoint independent sets will be ensured via the notion of alternating subsequences, which has been useful in, e.g., other similar contexts. A sequence of elements in $\{+,-, 0\}^{n}$ is alternating if all terms are nonzero and any two consecutive terms are different. Given an $\boldsymbol{x}=\left(x_{1}, \ldots, x_{n}\right) \in\{+,-, 0\}^{n}$, we denote by alt $(\boldsymbol{x})$ the maximum length of an alternating subsequence of $x_{1}, \ldots, x_{n}$.

The definition of the map $\lambda$ to which we will apply the octahedral Tucker lemma requires the quantity $s=\max \left\{\operatorname{alt}(\boldsymbol{x}): \boldsymbol{x} \in\{+,-, 0\}^{n}\right.$ s.t. $\left.I(\boldsymbol{x})=\varnothing\right\}$, where

$$
\begin{aligned}
I(\boldsymbol{x})=\{i \in[t]: & \left|\boldsymbol{x}^{+} \cap U_{i}\right|=\left|\boldsymbol{x}^{-} \cap U_{i}\right|=\left|U_{i}\right| / 2 \\
& \text { or } \left.\max \left(\left|\boldsymbol{x}^{+} \cap U_{i}\right|,\left|\boldsymbol{x}^{-} \cap U_{i}\right|\right)>\left|U_{i}\right| / 2\right\} .
\end{aligned}
$$

Note that $s \geq 0$.

Consider a nonzero vector $\boldsymbol{x} \in\{+,-, 0\}^{n}$. We distinguish two cases. In the case where $I(\boldsymbol{x}) \neq \varnothing$, we set $\lambda(\boldsymbol{x})= \pm\left(s+i^{\prime}\right)$, where $i^{\prime}$ is the maximum element in $I(\boldsymbol{x})$ and where the sign is defined as follows. When $\left|\boldsymbol{x}^{+} \cap U_{i^{\prime}}\right|=\left|\boldsymbol{x}^{-} \cap U_{i^{\prime}}\right|=$ $\left|U_{i^{\prime}}\right| / 2$, the sign is + if $\min \left(\boldsymbol{x}^{+} \cap U_{i^{\prime}}\right)<\min \left(\boldsymbol{x}^{-} \cap U_{i^{\prime}}\right)$ and is - otherwise. When $\max \left(\left|\boldsymbol{x}^{+} \cap U_{i^{\prime}}\right|,\left|\boldsymbol{x}^{-} \cap U_{i^{\prime}}\right|\right)>\left|U_{i^{\prime}}\right| / 2$, the sign is + if $\left|\boldsymbol{x}^{+} \cap U_{i^{\prime}}\right|>\left|U_{i^{\prime}}\right| / 2$, and it is - otherwise. In the case where $I(\boldsymbol{x})=\varnothing$, we set $\lambda(\boldsymbol{x})= \pm \operatorname{alt}(\boldsymbol{x})$, where the sign is the first nonzero element of $\boldsymbol{x}$. As in the proofs of Theorems 4.9 and [5.5, it can be checked that the map $\lambda$ satisfies the condition of the octahedral Tucker lemma with $m=s+t$. Thus we have $s+t \geq n$ and there exists $\boldsymbol{z}^{\prime} \in\{+,-, 0\}^{n}$ such that $I\left(\boldsymbol{z}^{\prime}\right)=\varnothing$ and $\operatorname{alt}\left(\boldsymbol{z}^{\prime}\right) \geq n-t$. It implies that there exists $\boldsymbol{z} \in\{+,-, 0\}^{n}$ such that $I(\boldsymbol{z})=\varnothing$ and $\operatorname{alt}(\boldsymbol{z})=\left|\boldsymbol{z}^{+}\right|+\left|\boldsymbol{z}^{-}\right|=n-t$. Let $A=\boldsymbol{z}^{+}$and $B=\boldsymbol{z}^{-}$. They are both independent sets. Since $I(\boldsymbol{z})=\varnothing$, we have $\left|A \cap U_{i}\right|+\left|B \cap U_{i}\right| \leq\left|U_{i}\right|-1$ for all $i$. The fact that $|A|+|B|=n-t$ then leads to $\left|A \cap U_{i}\right|+\left|B \cap U_{i}\right|=\left|U_{i}\right|-1$ for all $i$, and the conclusion follows.

Many statements about independent sets have analogues in terms of matchings, where a matching in a graph is a set of disjoint edges. This is natural since the matchings of a graph $G$ are the independent sets of its line graph, that is the graph in which vertices are the edges of $G$, and where edges with a common endpoint are connected. Matchings are important for theory and applications (see [249] for an excellent book about matchings). For instance, many resource management problems, take after the following example. Given a set of tasks and a set of workers, and a list of compatible workers for each task, assign to each task a different worker or report that no such assignment exists. The worker/task compatibilities can be modeled by a bipartite graph, so the question is whether there exists a matching that covers the vertex class modeling the tasks.

Colorful matchings still raise many questions, for instance the following wellknown conjecture due to Brualdi [82] and Stein [362].

Conjecture 5.6. If the edge set of $K_{n, n}$ (a complete bipartite graph with $n$ vertices in each side) is partitioned into sets $E_{1}, \ldots, E_{n}$ of size $n$ each, then there exists a matching in $K_{n, n}$ consisting of one edge from all but possibly one $E_{i}$.

A famous conjecture of Ryser about Latin squares 333. asserts that if $n$ is odd, then under the same condition as the Brualdi-Stein conjecture, there exists a perfect matching intersecting each $E_{i}$ once. 
5.3. Kernels in graphs. A kernel in a directed graph is a subset $K$ of the vertices that is independent (no two vertices of $K$ are joined by an arc) and absorbing (every vertex $v \notin K$ has an outgoing arc $v \rightarrow u$ to a vertex $u \in K$ ). Kernels naturally arise in certain combinatorial games, where they model the set of winning positions [387, or in stable matchings: it turns out the stable matchings of a graph with preferences equal the set of kernels of the associated line graph. Kernels proved effective in revisiting classical questions and are, for instance, at the heart of the proof by Galvin of Dinitz's conjecture on list colorings [10, §33].

Not every directed graph has a kernel (consider a directed cycle of length 3 ); this is in sharp contrast with the nondirected case, where the independent absorbing sets are the inclusion-maximal independent sets. As shown by a series of works by Richardson, Duchet, Meyniel, Galeana-Sánchez, and Neumann-Lara [147, 149, 178, 323 , a sufficient condition for the existence of a kernel is that each odd directed cycle has two chords whose heads are two consecutive vertices of the cycle. In particular, any acyclic directed graph has a kernel; this situation is actually what motivated, in the context of combinatorial games [387, the introduction of kernels. In general, however, deciding if a directed graph has a kernel is NP-complete [108].

Sperner's lemma comes up in the following relation between kernels and perfect graphs. A graph is perfect if for all its induced subgraphs, including itself, the chromatic number is equal to the clique number. These are precisely the graphs for which the trivial lower bound on the chromatic number is sharp for the graphs and all their induced subgraphs. The relation between kernels and perfect graphs is a special case of a conjecture of Berge and Duchet [57] proved by Boros and Gurvich 74].

Theorem 5.7. Any orientation of a perfect graph with no directed cycle of length three has a kernel.

The original proof translates any directed graph into a coalitional game, where the players are the cliques, and the outcomes are the stable sets. Under the theorem's assumptions, the game has stability properties that ensure, via results from coalitional game theory, the existence of a nonrejecting outcome, which translates into the desired kernel. A simpler and much more direct approach based on Scarf's lemma was proposed by Aharoni and Holzman [8] and was further simplified by Király and Pap [232] using Sperner's lemma.

The proof by Király and Pap goes as follows. Consider an orientation $D=(V, A)$ of a perfect graph with no directed cycle of length 3 . Let $P \subseteq \mathbb{R}^{V}$ denote the polyhedron of (possibly negative) vertex weights summing to at most 1 on every clique. This polyhedron has at least one extreme point (assigning 1 to any maximal independent set of $D$ and 0 to the remaining vertices does the job), so it is pointed. Moreover, it has exactly $n$ independent extreme directions (the $-\boldsymbol{e}_{v}$ where $\boldsymbol{e}_{v}$ is the unit vector associated to vertex $v$ ). Every facet of $P$ corresponds to a clique on which the weights sum to exactly 1 . Since there is no directed cycle of length 3 , every clique has a source, i.e., a vertex that is absorbed by all other vertices of the clique. Label each facet by the source of the corresponding clique. Note that a facet containing $-\boldsymbol{e}_{v}$ is not labeled by $v$. By Corollary 2.7, the polyhedron has an extreme point $\boldsymbol{\omega}$ that is incident to facets of each label. Now, consider the weights on $V$ defined by $\boldsymbol{\omega}$. If we could find an independent set $K$ of $D$ intersecting every clique of weight 1 , this set $K$ would also be absorbing: indeed, every vertex $v$ labels a facet incident to $\boldsymbol{\omega}$, so it is a source of a clique of weight 1 which intersects $K$. 
The existence of $K$ follows from a classical lemma of Lovász: in a perfect graph, there exists an independent set intersecting every clique of maximum weight. (This commonly used lemma is perhaps difficult to find spelled out in this form. A standard way to prove it is to use perfectness and coloring for cliques of maximum cardinality, then apply the vertex replication lemma of perfect graphs [246], to allow rational weights, then generalize to real weights using linear programming.)

Theorem 5.7 ensures the existence of a kernel, but the proof does not give any efficient method to compute one.

Open Problem 5.8. What is the complexity of computing a kernel in an orientation of a perfect graph with no directed cycle of length three?

\section{Optimization}

Broadly speaking, mathematical optimization develops mathematical tools for solving optimization problems. In this section we illustrate how the theorems of Carathéodory, Sperner, and Helly and their variations provide original viewpoints on different aspects of this field.

6.1. Linear programming. A linear program (LP) asks for the minimum of a linear function under a set of linear constraints and is usually written

$$
\begin{array}{cl}
\min & \boldsymbol{c}^{T} \boldsymbol{x} \\
\text { s.t. } & A \boldsymbol{x}=\boldsymbol{b}, \\
& \boldsymbol{x} \geq \mathbf{0} .
\end{array}
$$

Here, $A$ is a $m \times n$ matrix, $\boldsymbol{x}$ is a vector of $n$ indeterminates, $\boldsymbol{b}$ and $\boldsymbol{c}$ are vectors in $\mathbb{R}^{m}$ and $\mathbb{R}^{n}$, respectively, and $\boldsymbol{x} \geq \mathbf{0}$ means that each row of $\boldsymbol{x}$ is nonnegative. Linear programs may come in different presentations, with max in place of min or possibly inequalities in place of equalities; these presentations are essentially equivalent 262, $\S 4]$. Linear programming is by now a central tool in operations research as it allows researchers to model a variety of resource management problems [262, §2] and can be solved fairly effectively in practice. The theory of linear programming builds on the study of systems of linear inequalities. While this seems to be just a small variation from linear algebra, linear programming was only systematized in the late 1940s.

6.1.1. The simplex algorithm. Carathéodory's theorem underlies the simplex algorithm, arguably the standard method for solving linear optimization problems.

On the one hand, Carathéodory's theorem gives a way to discretize an a priori continuous problem. Indeed, the cone version of Carathéodory's theorem ensures that if the system $A \boldsymbol{x}=\boldsymbol{b}$ with $\boldsymbol{x} \geq \mathbf{0}$ admits a solution, then it admits a solution with support of size the rank of $A$. Such a support, understood as a set of indices of columns of $A$, is a feasible basis. A closer inspection of the proof of Proposition 3.1 reveals that the optimum of (6.1), if one exists, is attained on a solution supported by a feasible basis. Since a feasible basis determines a unique solution of $A \boldsymbol{x}=\boldsymbol{b}$, the optimum can be found in finite (but possibly long) time by enumerating feasible bases which are combinatorially described by their support.

On the other hand, Carathéodory's theorem, in the form of Proposition 3.1 also explains the pivoting mechanics of the simplex algorithm. Suppose there exists an optimum, and that we have a feasible basis $B$ determining a solution $\boldsymbol{x}^{*}$. It turns 
out that if $\boldsymbol{x}^{*}$ is not optimal, there exists $i \notin B$ such that increasing $x_{i}^{*}$ improves (i.e., decreases) the objective $\boldsymbol{c}^{T} \boldsymbol{x}$. The set $B \cup\{i\}$ contains another feasible basis, and it cannot define a worse solution than $\boldsymbol{x}^{*}$ (again, a consequence of the proof of Proposition 3.1). Switching to that new basis is a pivot step. It is a nontrivial result of Bland that there exist rules for choosing noncycling sequences of pivot steps; see [344, §11.3] and [262, §5.8]. Broadly speaking, the simplex algorithm starts by computing a feasible basis, and then it performs such pivot steps until no entry outside the basis can be used to improve the objective; the final basis then determines an optimal solution.

6.2. Integer programming. An integer program (IP) adds integrality constraints to linear programs by restricting all of the variables to take their values over $\mathbb{Z}$ rather than $\mathbb{R}$, for instance

$$
\begin{aligned}
\min & \boldsymbol{c}^{T} \boldsymbol{x} \\
\text { s.t. } & A \boldsymbol{x}=\boldsymbol{b}, \\
& \boldsymbol{x} \geq \mathbf{0}, \boldsymbol{x} \in \mathbb{Z}^{n} .
\end{aligned}
$$

This variation arises naturally in the management of indivisible resources or yes/no decision making; the emblematic example is the knapsack problem which asks, given a set of objects with weights and values, for the subset of maximal value whose weight does not exceed a given threshold.

The relaxation of an integer linear program is the linear program obtained by forgetting the integrality conditions, as is (6.1) for (6.2). In general, the solution to the relaxed linear program provides a bound on the solution to the integer program, a lower bound in the case of (6.1) and (6.2). Linear programming and relaxation play a fundamental role in combinatorial algorithms; we refer the reader to the books 385, 391 for more detail.

What we just said applies also on mixed integer programs in which only some of the variables are required to be integers.

6.2.1. Sparsity of integer solutions. Carathéodory's theorem readily measures the sparsity of optimal solutions. For example, Theorem 3.2 provides the following bound on the support of an optimal solution.

Corollary 6.1. Let $A \in \mathbb{Z}^{m \times d}, \boldsymbol{b} \in \mathbb{Z}^{m}$, and $\boldsymbol{c} \in \mathbb{Z}^{d}$. The integer point of the polyhedron $\left\{\boldsymbol{x} \in \mathbb{R}^{d}: A \boldsymbol{x}=\boldsymbol{b}, \boldsymbol{x} \geq \mathbf{0}\right\}$ that minimizes $\boldsymbol{c}^{T} \boldsymbol{x}$ has at most

$$
2(m+1) \log (2 \sqrt{(m+1)} M)
$$

nonzero components, where $M$ is the largest of the entries of $A, c$ in absolute value.

Similar results have been used, for instance, for solving of bin-packing problems; see e.g., [155, 189]. See [13] for an application to the sparsity of optimal solutions and tighter bounds for special cases such as knapsack problems.

6.2.2. Graver bases. Another example of the influence of Carathéodory's theorem is the use of Hilbert bases by Graver's optimization methods. Although we present these ideas for integer programs, they apply more broadly, for instance to convex integer optimization problems, with respect to a convex objective function composed with linear functions, or convex separable functions; see [124, $\S 3$ and $\S 4$ ] and [309]. 

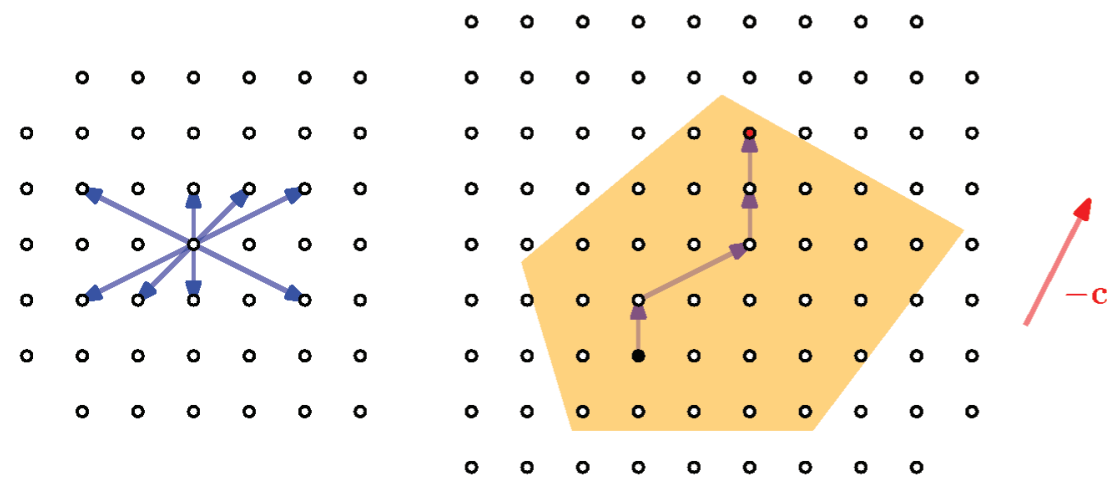

Figure 13. A planar projection illustration of Graver basis methods for $A \boldsymbol{x}=\boldsymbol{b}$ for $A=\left(\begin{array}{lll}1 & 2 & 1\end{array}\right)$. The Graver basis of $A \boldsymbol{x}=\mathbf{0}$ consists of $(2,-1,0),(0,-1,2),(1,0,-1),(1,-1,1)$, and their opposites. On the left, the neighbors of an integer point through the Graver basis. On the right, the cone $\boldsymbol{x} \geq 0$ (shaded) and a walk from an arbitrary (black) feasible integer point to the (red) integer point optimal for the given direction $\boldsymbol{c}$.

Consider the integer program (6.2) and assume, as is usually the case in practice, that $A \in \mathbb{Q}^{m \times n}$. We can decompose the polyhedron $A \boldsymbol{x}=\mathbf{0}$ into $2^{n}$ cones,

$$
\{A \boldsymbol{x}=\mathbf{0}\}=\bigcup_{\boldsymbol{\varepsilon} \in\{-1,1\}^{n}}\left\{A \boldsymbol{x}=\mathbf{0}, \varepsilon_{1} x_{1} \geq 0, \varepsilon_{2} x_{2} \geq 0, \ldots, \varepsilon_{n} x_{n} \geq 0\right\}
$$

see the left-hand side of Figure 13, Each of these cones is pointed and rational, so it has a Hilbert basis [124, Corollary 2.6.4]. The union of these $2^{d}$ Hilbert bases is called the Graver basis of $A$. Note that Sebő's integer Carathéodory theorem (Theorem 3.3) ensures that any integer point in the polyhedron $A \boldsymbol{x}=\boldsymbol{b}$ can be written as a nonnegative integer linear combination of at most $2 n-2$ vectors from the Graver basis of $A$. Moreover, Graver [192] established the following remarkable augmentation property: any nonoptimal feasible solution of the integer program (6.2) can be improved by adding some suitable vector from the Graver basis of $A$. Hence, any integer program of the form (6.2) can be solved by first computing a Graver basis for $A \boldsymbol{x}=\boldsymbol{b}$, then computing a feasible solution, and finally improving this solution by a greedy walk on the set of integer solutions, the candidate steps being provided by the vectors of the Graver basis; see the right-hand side of Figure 13 .

The main obstacle to the practical use of Graver bases is their potentially exponential size. For general matrices, deciding if a given set of vectors is a Hilbert basis is already co-NP-complete [150. The good news, from the last decade of work, is that for highly structured matrices, such as those with regular block decompositions, Graver bases can be computed efficiently and are actually manageable for optimization (see details in [124, Chapters 3 and 4] and the extensive presentation in 309]).

6.3. LP duality. An important idea for the study of linear programs is the notion of LP duality. This idea naturally arises from the question of certifying the quality of a solution to a linear program. For example, the objective function value of an 
optimal solution to

$$
\begin{array}{ll}
\min & 5 x_{1}+3 x_{2} \\
\text { s.t. } & \left(\begin{array}{ll}
2 & 3 \\
1 & 2
\end{array}\right)\left(\begin{array}{l}
x_{1} \\
x_{2}
\end{array}\right) \geq\left(\begin{array}{l}
2 \\
5
\end{array}\right), \\
& \boldsymbol{x} \geq \mathbf{0}
\end{array}
$$

can be seen to be at least 2 by looking at the first constraint and at least $\frac{15}{2}$ by scaling the second constraint by $\frac{3}{2}$. More generally, the solution of a linear program $(\mathrm{P})$ can be bounded from below by the solution of a linear program (D), where

$$
\begin{aligned}
& \text { (P) } \min \boldsymbol{c}^{T} \boldsymbol{x} \\
& \text { s.t. } \quad A \boldsymbol{x} \geq \boldsymbol{b}, \quad \text { and } \\
& \boldsymbol{x} \geq \mathbf{0} \\
& \text { (D) } \max \boldsymbol{b}^{T} \boldsymbol{y} \\
& \text { s.t. } A^{T} \boldsymbol{y} \leq \boldsymbol{c} \text {, } \\
& \boldsymbol{y} \geq \mathbf{0} \text {. }
\end{aligned}
$$

The linear programs $(\underline{\mathrm{P}})$ and $(\mathrm{D})$ are said to be dual to one another; the variable $\boldsymbol{y}$ of the dual program can be interpreted as the weights of a linear combination of the constraints of the primal program (and conversely). This relation, called weak linear programming duality, can be strengthened.

Theorem 6.2 (Strong duality). Given two dual linear programs, if at least one is feasible, then they have the same optimal value.

The duality theory of linear optimization has many applications, such as fast certification of solutions or primal-dual algorithms and in proving combinatorial theorems 344. But it also plays a role in discrete geometry, for example in the proof of $(p, q)$ theorems [22. In the following subsection we are going to prove Theorem 6.2 in an atypical way.

6.3.1. LP duality from the MinMax theorem. LP duality is the classical favorite approach to prove von Neumann's MinMax theorem (Theorem 4.5) for two-player zero-sum games, as we mentioned in Section 4.1.2. Going in the other direction, Dantzig [119] proposed a deduction of the strong duality theorem from the MinMax theorem; as we explain below, Dantzig's proof required a detour, in some cases, via Farkas's lemma, another result equivalent to Theorem 6.2. The impression of equivalence between the MinMax theorem, Nash equilibria for zero-sum two-player games, and the strong duality theorem nevertheless lingered and became a folklore theorem. It is only recently that Adler [5] filled in the missing case to give a genuine direct equivalence between these three cornerstones.

Dantzig's approach proceeds as follows. The weak duality already proves one inequality. The other inequality reduces to finding a solution $(\boldsymbol{x}, \boldsymbol{y}) \geq 0$ of the system $(\underline{\mathrm{P}+\mathrm{D})})$ :

$$
\left\{\begin{aligned}
A \boldsymbol{x}-\boldsymbol{b} & \geq 0 \\
-A^{T} \boldsymbol{y}+\boldsymbol{c} & \geq 0 \\
\boldsymbol{b}^{T} \boldsymbol{y}-\boldsymbol{c}^{T} \boldsymbol{x} & \geq 0
\end{aligned}\right.
$$


This system rewrites

$$
\underbrace{\left(\begin{array}{ccc}
0 & A & -\boldsymbol{b} \\
-A^{T} & 0 & \boldsymbol{c} \\
\boldsymbol{b}^{T} & -\boldsymbol{c}^{T} & 0
\end{array}\right)}_{M}\left(\begin{array}{l}
\boldsymbol{y} \\
\boldsymbol{x} \\
1
\end{array}\right) \geq \mathbf{0}
$$

so our task is to find a vector $\boldsymbol{z}$ with positive last component and such that $M \boldsymbol{z} \geq \mathbf{0}$. Consider the zero-sum game with payoff matrix $-M$, and let $\left(\boldsymbol{s}^{*}, \boldsymbol{t}^{*}\right)$ be a Nash equilibrium. The matrix $-M$ has dimension $n \times n$, so one may pit each strategy against itself to see that the value $v$ of the game satisfies

$$
\boldsymbol{s}^{* T}(-M) \boldsymbol{s}^{*} \geq v \geq \boldsymbol{t}^{* T}(-M) \boldsymbol{t}^{*} .
$$

Since $M$ is antisymmetric, this implies that $v=0$. Moreover, for any $\boldsymbol{z} \in \Delta_{n-1}$, we have $\boldsymbol{s}^{* T}(-M) \boldsymbol{z} \geq 0$, so $M \boldsymbol{s}^{*} \geq \mathbf{0}$. Since $\boldsymbol{s}^{*} \in \Delta_{n-1}$, writing $\boldsymbol{s}^{*}=\left(\begin{array}{lll}\widetilde{\boldsymbol{y}} & \widetilde{\boldsymbol{x}} & \widetilde{u}\end{array}\right)^{T}$ leads to the desired solution whenever $\widetilde{u} \neq 0$. When $\widetilde{u}=0$, Dantzig concluded by a separate use of Farkas's lemma (the incompleteness in his derivation). Adler was able to complete this missing case, without appealing to Farkas.

6.3.2. Totally dual integral polyhedra. Applied optimization models typically involve rational polyhedra, which are expressed as systems of linear inequalities with rational coefficients. An important question for computation is whether a rational polyhedron is integral, that is whether all its vertices have integer coordinates. Indeed, for integral polyhedra, integer optimization (which is typically very hard) becomes linear optimization (which is considered tractable). Let us see how Hilbert bases help when looking for rational polyhedra that are integral. In what follows, we consider a rational polyhedron $P=\{\boldsymbol{x}: A \boldsymbol{x} \leq \boldsymbol{b}\}$ with $A$ and $\boldsymbol{b}$ rational.

Checking whether $P$ is integral is a finite process, as one can simply list all the vertices. The following structural result allows us to bypass this tedious enumeration in various situations. Observe that if $P$ is integral, then for every integral vector $\boldsymbol{w}$, the value $\max \left\{\boldsymbol{w}^{T} \boldsymbol{x}: \boldsymbol{x} \in P\right\}$ is an integer (indeed, it is the inner product of two integral vectors). Surprisingly, a rational polyhedron is integral if and only if it satisfies this condition. This equivalence, due to Edmonds and Giles 154, is still not a practical way to detect integral polyhedra (the set of candidate vectors $\boldsymbol{w}$ is infinite) but it suggests that we look at things via duality. Indeed, the strong LP duality (Theorem 6.2) states

$$
\max \left\{\boldsymbol{w}^{T} \boldsymbol{x}: A \boldsymbol{x} \leq \boldsymbol{b}\right\}=\min \left\{\boldsymbol{y}^{T} \boldsymbol{b}: A^{T} \boldsymbol{y}=\boldsymbol{w}, \boldsymbol{y} \geq 0\right\},
$$

so $P$ is integral if the vector $\boldsymbol{b}$ is integral and the right-hand side minimization problem has an integral optimal value for every integral vector $\boldsymbol{w}$. (Note that, in general, integrality properties are not preserved through linear programming duality.) A system of inequalities $A \boldsymbol{x} \leq \boldsymbol{b}$ is totally dual integral (TDI) if the righthand side minimization problem above has an integral solution for every integral vector $\boldsymbol{w}$ (for which the optimum is finite). A rational polyhedron $P$ that can be represented by a TDI system where $\boldsymbol{b}$ is integral is thus integral. The converse is true and any integral polyhedron can be represented by a TDI system of inequalities (but which, in practice, may not be easy to find). Let us stress that TDI-ness is a property of the system of inequalities, not of the underlying polyhedron: Giles and Pulleyblank [187] proved that for every rational system of inequalities $A \boldsymbol{x} \leq \boldsymbol{b}$, there is a rational number $\alpha$ such that $\alpha A \boldsymbol{x} \leq \alpha \boldsymbol{b}$ is TDI. They also proved, using 
Carathéodory-style properties, that a system of inequalities $A \boldsymbol{x} \leq \boldsymbol{b}$ is TDI if and only if for every face $F$ of $P$, the rows of $A$ which are active in $F$ form a Hilbert basis for that supporting cone. This makes checking TDI-ness a finite process, but it is still not a practical one as checking whether a system of vectors forms a Hilbert basis is not efficient. See [150] and references therein for computational issues.

TDI-ness and related notions, such as box-TDI-ness, often shed new light on results in combinatorial optimization, for instance on the matroid intersection theorem. Consider two matroids $M_{1}$ and $M_{2}$ over the same ground set $S$, understood as their sets of independent sets. Any matroid has an associated matroid polytope, obtained by taking the convex hull of its (indicator vectors of) independent sets. It turns out that the convex hull of the independent sets in $M_{1} \cap M_{2}$ coincides with the intersection of the matroid polytopes of both matroids. This is remarkable, as in general $\operatorname{conv}(A \cap B)$ is different from $\operatorname{conv}(A) \cap \operatorname{conv}(B)$; see [345, §41] for more on this topic.

A special case of TDI-ness allows for linear programming proofs of combinatorial results. A matrix $A$ is totally unimodular (TU) if every square submatrix has determinant in $\{0,-1,+1\}$. For such a matrix, the polyhedron $\{\boldsymbol{x}: A \boldsymbol{x} \leq \boldsymbol{b}, \boldsymbol{x} \geq$ $\mathbf{0}\}$ is integral for every integral vector $\boldsymbol{b}$. TU matrices give rise to TDI systems. They are completely characterized and are very important in combinatorics and optimization (see 350,390 ). Since the transpose of a TU matrix is TU again, the strong duality theorem in linear programming (Theorem 6.2) provides alternative proofs of Konnig's theorem (the maximum cardinality of a matching in a bipartite graph is equal to the minimum cardinality of a set of nodes intersecting each edge), the König-Rado theorem (the maximum cardinality of a stable set in a bipartite graph without isolated vertices is equal to the minimum number of edges needed to cover all nodes), and the integrality of the max-flow/min-cut theorem. In all three cases, the matrix $A$ is the node-arc incidence matrix of a directed graph, and it is an easy exercise to check that such a matrix is TU.

6.4. Convex optimization. Using linearization techniques, one may apply ideas from the theory of linear programming, and its duality, to more general optimization problems of the form

$$
\begin{array}{cl}
\min & f(\boldsymbol{x}) \\
\mathrm{s.t.} & h_{j}(\boldsymbol{x}) \leq 0, \quad j=1, \ldots, q,
\end{array}
$$

where $f$ and $h_{j}$ 's are differentiable functions $\mathbb{R}^{n} \rightarrow \mathbb{R} \cup\{-\infty,+\infty\}$ (not just linear as before).

6.4.1. The KKT conditions from LP duality. A milestone in mathematical programming is the following necessary optimality condition, due to Karush, Kuhn, and Tucker [76].

Theorem 6.3 (KKT condition). Let $\boldsymbol{x}^{*}$ be a feasible solution of the problem ( $\left(\mathrm{P}^{\prime}\right)$ such that the constraints are qualified at $\boldsymbol{x}^{*}$. If $\boldsymbol{x}^{*}$ is a local optimum, then there are nonnegative real numbers $\mu_{1}, \mu_{2}, \ldots, \mu_{q}$ such that

$$
\nabla f\left(\boldsymbol{x}^{*}\right)+\sum_{j=1}^{q} \mu_{j} \nabla h_{j}\left(\boldsymbol{x}^{*}\right)=\mathbf{0} \quad \text { and } \quad \forall j, \quad \mu_{j} h_{j}\left(\boldsymbol{x}^{*}\right)=0 .
$$

The requirement that the "constraints are qualified" is a regularity condition on the feasible domain $F=\left\{\boldsymbol{x}: h_{j}(\boldsymbol{x}) \leq 0, j \in[q]\right\}$ near $\boldsymbol{x}^{*}$. We do not spell out 
this rather technical condition, but we will give a sufficient requirement. Call a direction $\boldsymbol{d}$ feasible at $\boldsymbol{x}^{*}$ if $F$ contains a segment of positive length with endpoint $\boldsymbol{x}^{*}$ and direction $\boldsymbol{d}$. (This is where we are approximating: the adequate notion of feasibility is somewhat more flexible.) The constraints are qualified at $\boldsymbol{x}^{*}$ if the closure of the cone of feasible directions coincides with the tangent cone at $\boldsymbol{x}^{*}$, that is

$$
\left\{\boldsymbol{d}: \nabla h_{j}\left(\boldsymbol{x}^{*}\right) \cdot \boldsymbol{d} \leq 0 \quad \text { for all } j \in[q] \text { s.t. } h_{j}\left(\boldsymbol{x}^{*}\right)=0\right\} .
$$

Even this coarser requirement may prove tedious to check, and several simpler sufficient conditions were investigated. The above criterion readily yields that affine constraints are qualified in any feasible point. Another important case is that of convex differentiable constraints, which are qualified at any feasible point provided there exists a point satisfying strictly every constraint [76, §5.5].

The factors $\mu_{i}$ in Theorem 6.3 are the Lagrange multipliers. The strong duality theorem is classically equivalent to the following lemma of Farkas [344, $\S 7.3]$.

Lemma 6.4 (Farkas's lemma). Let $A$ be a real matrix, and let $\boldsymbol{b}$ be a vector. There exists $\boldsymbol{x} \geq \mathbf{0}$ such that $A \boldsymbol{x}=\boldsymbol{b}$ if and only if $\boldsymbol{y} \cdot \boldsymbol{b} \geq 0$ for every $\boldsymbol{y}$ such that $A^{T} \boldsymbol{y} \geq \mathbf{0}$.

Theorem 6.3 can be deduced from Farkas's lemma via the following linearization argument. Let $\boldsymbol{x}^{*}$ be a feasible solution of $\left(\underline{\mathrm{P}^{\prime}}\right)$, and write

$$
A=-\left(\left(\nabla h_{j}\left(\boldsymbol{x}^{*}\right)\right)_{j \in J}\right) \quad \text { where } \quad J=\left\{j \in[q]: h_{j}\left(\boldsymbol{x}^{*}\right)=0\right\} .
$$

Let $\boldsymbol{x}^{*}$ be a local optimum of $\left(\underline{\mathrm{P}^{\prime}}\right)$. Since $f$ is differentiable, $\nabla f\left(\boldsymbol{x}^{*}\right) \cdot \boldsymbol{d} \geq 0$ for every direction $\boldsymbol{d}$ feasible at $\boldsymbol{x}^{*}$. Moreover, since the constraints are qualified at $\boldsymbol{x}^{*}$, any direction $\boldsymbol{d}$ satisfying $A^{T} \boldsymbol{d} \geq \mathbf{0}$ is in the closure of the cone of directions feasible at $\boldsymbol{x}^{*}$, hence

$$
\forall \boldsymbol{d} \in \mathbb{R}^{n} \text { s.t. } A^{T} \boldsymbol{d} \geq \mathbf{0}, \quad \nabla f\left(\boldsymbol{x}^{*}\right) \cdot \boldsymbol{d} \geq 0 .
$$

By Farkas's lemma, this is equivalent to the existence of a vector $\boldsymbol{\mu}^{\prime}$ in $\mathbb{R}_{+}^{J}$ such that $A \boldsymbol{\mu}^{\prime}=\nabla f\left(\boldsymbol{x}^{*}\right)$. Completing $\boldsymbol{\mu}^{\prime}$ into $\boldsymbol{\mu}$ by zeroes yields Theorem 6.3.

6.4.2. Strong duality in convex programming from the KKT conditions. The KKT condition (Theorem 6.3) can, in turn, be used to prove a strong duality theorem for convex programming, generalizing Theorem 6.2. Let us introduce the Lagrangian function

$$
\mathcal{L}(\boldsymbol{x}, \boldsymbol{\mu})=f(\boldsymbol{x})+\sum_{j=1}^{q} \mu_{j} h_{j}(\boldsymbol{x})
$$

Since

$$
\sup _{\boldsymbol{\mu} \in \mathbb{R}_{+}^{q}} \mathcal{L}(\boldsymbol{x}, \boldsymbol{\mu})= \begin{cases}f(\boldsymbol{x}) & \text { if } \mu_{j} h_{j}(\boldsymbol{x}) \leq 0, \\ +\infty & \text { otherwise. }\end{cases}
$$

$\left(\mathrm{P}^{\prime}\right)$ is equivalent to

$$
\begin{array}{cl}
\min & \sup \left\{\mathcal{L}(\boldsymbol{x}, \boldsymbol{\mu}): \boldsymbol{\mu} \in \mathbb{R}_{+}^{q}\right\} \\
\text { s.t. } & \boldsymbol{x} \in \mathbb{R}^{n} .
\end{array}
$$

The same argument as in equation (4.4) yields

$$
\inf _{\boldsymbol{x} \in \mathbb{R}^{n}} \sup \left\{\mathcal{L}(\boldsymbol{x}, \boldsymbol{\mu}): \boldsymbol{\mu} \in \mathbb{R}_{+}^{q}\right\} \geq \sup _{\mu \in \mathbb{R}_{+}^{q}} \inf \left\{\mathcal{L}(\boldsymbol{x}, \boldsymbol{\mu}): \boldsymbol{x} \in \mathbb{R}^{n}\right\} .
$$


Finding the right-hand side term consists in solving the following dual program:

$$
\begin{aligned}
\max & g(\boldsymbol{\mu}) \quad \text { where } \quad g(\boldsymbol{\mu})=\inf \left\{\mathcal{L}(\boldsymbol{x}, \boldsymbol{\mu}): \boldsymbol{x} \in \mathbb{R}^{n}\right\} \\
\text { s.t. } & \boldsymbol{\mu} \geq \mathbf{0} .
\end{aligned}
$$

This dual program always asks us to maximize a concave function. In the case where problem $\left(\mathrm{P}^{\prime}\right)$ is a linear program, this notion of duality coincides with the LP duality introduced in Section 6.3.

Proposition 6.5 (Strong duality for convex optimization). Suppose that in $\left(\mathrm{P}^{\prime}\right)$, $f$ and $h_{1}, \ldots, h_{q}$ are convex functions. Suppose moreover that the constraints are qualified at every feasible solution. If $\left(\mathrm{P}^{\prime}\right)$ has an optimal solution, then the dual program has one also, and the optimal values of $\left(\mathrm{P}^{\prime}\right)$ and $\left(\mathrm{D}^{\prime}\right)$ coincide.

The proof goes as follows. Let $\boldsymbol{x}^{*}$ be an optimal solution of $\left(\mathrm{P}^{\prime}\right)$. By Theorem 6.3 , there exists $\boldsymbol{\mu}^{*} \in \mathbb{R}_{+}^{q}$ such that

$$
\nabla f\left(\boldsymbol{x}^{*}\right)+\sum_{j=1}^{q} \mu_{j}^{*} \nabla h_{j}\left(\boldsymbol{x}^{*}\right)=\mathbf{0} \quad \text { and } \quad \forall j, \quad \mu_{j}^{*} h_{j}\left(\boldsymbol{x}^{*}\right)=0 .
$$

On the one hand, we have

$$
\mathcal{L}\left(\boldsymbol{x}^{*}, \boldsymbol{\mu}^{*}\right)=f\left(\boldsymbol{x}^{*}\right)+\sum_{j=1}^{q} \mu_{j}^{*} h_{j}\left(\boldsymbol{x}^{*}\right)=f\left(\boldsymbol{x}^{*}\right) .
$$

On the other hand, we have

$$
\nabla_{\boldsymbol{x}} \mathcal{L}\left(\boldsymbol{x}, \boldsymbol{\mu}^{*}\right)=\nabla f(\boldsymbol{x})+\sum_{j=1}^{q} \mu_{j}^{*} \nabla h_{j}(\boldsymbol{x}), \quad \text { so } \quad \nabla_{\boldsymbol{x}} \mathcal{L}\left(\boldsymbol{x}^{*}, \boldsymbol{\mu}^{*}\right)=\mathbf{0} .
$$

The map $\boldsymbol{x} \mapsto \mathcal{L}\left(\boldsymbol{x}, \boldsymbol{\mu}^{*}\right)$ is convex because $\boldsymbol{\mu}^{*} \geq \mathbf{0}$, so $\boldsymbol{x}^{*}$ is a global minimum and $g\left(\boldsymbol{\mu}^{*}\right)=\mathcal{L}\left(\boldsymbol{x}^{*}, \boldsymbol{\mu}^{*}\right)=f\left(\boldsymbol{x}^{*}\right)$. Together with the weak duality of equation (6.4), this ensures that $\boldsymbol{\mu}^{*}$ is an optimal solution for ( $\left.\mathrm{D}^{\prime}\right)$.

6.5. Sampling approaches. Let us now consider optimization problems of the form

$$
\begin{aligned}
\min & f(\boldsymbol{x}) \\
\text { s.t. } & \boldsymbol{x} \in C_{1} \cap C_{2} \cap \cdots \cap C_{m},
\end{aligned}
$$

where one minimizes a function over an intersection of subsets $C_{i}$, the constraints. Such problems include linear programming (when $f$ is linear and the $C_{i}$ are halfspaces), convex programming (when $f$ is convex and the $C_{i}$ are convex sets), or their integral or mixed analogues (via restrictions to $\mathbb{Z}^{k} \times \mathbb{R}^{d}$ ).

6.5.1. Witness sets of constraints. A first use of Helly's theorem concerns the removal of redundant constraints defining an optimal solution. To begin, consider the linear program

$$
\begin{array}{cl}
\min & \boldsymbol{c}^{T} \boldsymbol{x} \\
\text { s.t. } & A \boldsymbol{x} \leq \boldsymbol{b}, \\
& \boldsymbol{x} \in \mathbb{R}^{d},
\end{array}
$$

where $A \in \mathbb{R}^{m \times n}$ (this form differs from those seen so far but is equivalent [262, §4]). Assume that the problem is feasible, and let $t$ denote its solution. The set $A \boldsymbol{x} \leq \boldsymbol{b}$ of feasible solutions is an intersection of $m$ halfspaces, and their common intersection 
with $\boldsymbol{c}^{T} \boldsymbol{x}<t$, another halfspace, is empty. By Helly's theorem, some $d+1$ of these $m+1$ halfspaces must have empty intersection, and $\boldsymbol{c}^{T} \boldsymbol{x}<t$ must be one of them. It follows that we may drop from (6.6) all but some (carefully chosen) $d$ constraints without changing the solution. Recall that Helly's theorem for halfspaces is dual to Carathéodory's theorem; in the dual, this argument yields that an optimal solution can be realized by a feasible basis (as introduced in Section 6.1.1).

More generally, given a problem of the form (6.5), a subset $W \subseteq\left\{C_{1}, C_{2}, \ldots, C_{m}\right\}$ is a witness set of constraints if

$$
\begin{aligned}
& \min f(\boldsymbol{x})=\min f(\boldsymbol{x}) \\
& \text { s.t. } \boldsymbol{x} \in \bigcap_{i=1}^{m} C_{i} \quad \text { s.t. } \boldsymbol{x} \in \bigcap_{C \in W} C \text {, }
\end{aligned}
$$

and $W$ is inclusion-minimal for that property; in other words, a witness set is a nonredundant set of constraints that defines the same optimum as the entire problem. The above argument gives, mutatis mutandis, that the witness sets of any feasible mixed-convex programming over $\mathbb{Z}^{k} \times \mathbb{R}^{d}$ have size at most $2^{k}(d+1)-1$; this bound increases by 1 for unfeasible programs.

The relation between Helly's theorem and witness sets extends beyond convexity, as observed by Amenta [26]. We say that a family $\mathcal{F}$ admits a Helly-type theorem with constant $h$ if the nonempty intersection of every $h$-element subset of $\mathcal{F}$ implies the nonempty intersection of $\mathcal{F}$. Consider a minimization problem of the form (6.5), where $f$ is a function from a space $X$ to a space $V$. We assume that $V$ is equipped with a total order $\prec$, so that the minimization question makes sense. For any $v \in V$ we let $L_{v}=\{x \in X: f(x) \prec v\}$.

Proposition 6.6. Let $h \in \mathbb{N}$. If every family $\left\{C_{1}, C_{2}, \ldots, C_{m}, L_{v}\right\}$ admits a Hellytype theorem with constant $h$, then any witness set of constraints of (6.5) has size at most $h$ (actually, $h-1$ if the problem is feasible).

Note that we make no assumption on $f$ (not even continuity!), $X$, or $V$. The proof for the feasible case goes as follows. Let $\mathcal{F}=\left\{C_{1}, C_{2}, \ldots, C_{m}\right\}$, and let $s$ denote the solution to (6.5). For $G \subseteq\left\{C_{1}, C_{2}, \ldots, C_{m}\right\}$, define $s(G)$ as the minimum of $f$ over $\bigcap_{C \in G} C$, and put

$$
s^{\prime}=\max \{s(G): G \subseteq \mathcal{F} \text { and }|G|=h-1\} .
$$

On the one hand, $s \geq s(G)$ for every $G \subseteq \mathcal{F}$, we have $s \geq s^{\prime}$. On the other hand, every $h$ elements of $\mathcal{F}$ intersect (because (6.5) is feasible) and $L_{s^{\prime}}$ intersects any $h-1$ elements of $\mathcal{F}$ (by definition of $s^{\prime}$ ); thus, every $h$ elements in $\mathcal{F} \cup\left\{L_{s^{\prime}}\right\}$ intersect, and the Helly-type theorem on $\mathcal{F} \cup\left\{L_{s^{\prime}}\right\}$ ensures that $s \leq s^{\prime}$. By minimality, any witness set thus has size at most $h-1$.

6.5.2. Combinatorial algorithms for linear programming. Devising algorithms for linear programming with provably good complexity has been a major challenge for the past 70 years. The interior point method of Karmarkar 228 and the analysis of the ellipsoid method by Khachyian [231] only showed that the complexity of LP is polynomial in the number $m$ of constraints, the number $d$ of variables, and the bit complexity $L$ of the entries of the matrix $A$ and vectors $\boldsymbol{b}$ and $\boldsymbol{c}$. A major question thus remains: 
Open Problem 6.7. Is there an algorithm that solves linear programming in time polynomials in the number of constraints and the number of variables, assuming that arithmetic operations on input numbers have unit cost?

(This is problem number nine in Smale's list of open problems [355].) An algorithm with complexity polynomial in $m$ and $d$ in the unit cost model is called strongly polynomial. Although the simplex algorithm proves effective in practice, no choice of pivoting rule is known to ensure a number of step polynomial in the number $m$ of constraints and the number $d$ of variables; in fact, for every pivot rule whose worst-case complexity is established, that complexity is at least exponential in $m$ and $d$ (see [37, 118, 173, 233] and the references therein). Although no strongly polynomial time algorithm is known, partial progress was made through the $1980 \mathrm{~s}$ and 1990s via combinatorial random sampling algorithms; this approach hinges on the fact that the bounded size of witness sets allows us to throw away redundant constraints quickly.

Let us illustrate the basic idea of combinatorial random sampling algorithms in its simplest form, due to Seidel [349] (see also [123, §4]). Consider

$$
\begin{array}{cl}
\min & \boldsymbol{c}^{T} \boldsymbol{x} \\
\text { s.t. } & \boldsymbol{x} \in \mathbb{R}^{d}, \\
& \boldsymbol{x} \in H_{1} \cap H_{2} \cap \cdots \cap H_{m},
\end{array}
$$

where each $H_{i}$ is a halfspace in $\mathbb{R}^{d}$. Pick $t \in[m]$ uniformly at random, and let $\boldsymbol{s}_{t}$ denote the solution to the linear program with the constraint $H_{t}$ removed. The idea is to compute $s_{t}$ recursively, then deduce $s$ from $s_{t}$ : we check in $O(d)$ time whether $\boldsymbol{s}_{t}$ belongs to $H_{t}$. If $\boldsymbol{s}_{t} \in H_{t}$, then $\boldsymbol{s}=\boldsymbol{s}_{t}$, and if $\boldsymbol{s}_{t} \notin H_{t}$, then $\boldsymbol{s}$ must belong to the hyperplane bounding $H_{t}$ and be the solution to the linear program

$$
\begin{aligned}
\min & \boldsymbol{c}^{T} \boldsymbol{x} \\
\text { s.t. } & \boldsymbol{x} \in \partial H_{t}, \\
& \boldsymbol{x} \in \bigcap_{i \in[m] \backslash\{t\}}\left(H_{i} \cap \partial H_{t}\right) .
\end{aligned}
$$

This new linear program has $m-1$ constraints in $d-1$ variables and can be obtained from (6.7) in time $O(d m)$. Altogether, the expected time $T(m, d)$ to compute $s$ writes

$$
T(m, d)=T(m-1, d)+O(d)+\mathbb{1}_{\boldsymbol{s}_{t} \notin H_{t}} T(m-1, d-1) .
$$

Observe that $\boldsymbol{s}_{t} \notin H_{t}$ if and only if $H_{t}$ belongs to every witness set for (6.7). Since the size of witness sets is at most $d$, the event $s_{t} \notin H_{t}$ occurs with probability at most $\frac{d}{m}$ when $t$ is chosen uniformly at random. Altogether, this recursion solves to $T(m, d)=O(d ! m)$, which is the running time of Seidel's algorithm. In fact, Seidel's algorithm builds on an idea by Clarkson, which we describe next.

The iterated reweighting method of Clarkson [110] consists of assigning a weight $w_{i}$, initially set to 1 , to every constraint $H_{i}$ and iterating a simple process: Sample $O\left(d^{2}\right)$ constraints with probabilities proportional to their current weights and solve the problem on these constraints. If the solution is feasible, we are done; otherwise double the weights of all violated constraints, and reiterate. It remains to be shown that, almost surely, the algorithm terminates. This can be seen by comparing the growth rates of the total weight of the system, and the weights of some witness set $W$. On the one hand, every unsuccessful iteration must double the weight of at 
least one constraint in $W$. On the other hand, as the constraints are chosen in each iteration with probability proportional to their current weight, the expected total weight of the constraints violated at any given iteration is $O\left(\frac{\sum_{i} w_{i}}{d}\right)[94$. Thus, after $k$ iterations, $W$ has weight $\Omega\left(d 2^{k / d}\right)$ but the total weight of all the constraints in the system is $O\left(m\left(1+\frac{O(1)}{d}\right)^{k}\right)$. Putting these two bounds together implies that the algorithm terminates, with high probability, within $O(d \log m)$ iterations. At each iteration one has to solve a linear program on $O\left(d^{2}\right)$ constraints, which can be done in time $O(d)^{d / 2+O(1)}$, say by the simplex method, and compute the set of violating constraints, which takes time $O(m d)$. This implies an overall running time of $O\left(d^{2} m \log m+O(d)^{d / 2+O(1)} \log m\right)$.

Clarkson's approach was later improved by Matoušek et al. 264 to achieve an expected time complexity of $O\left(d^{2} m+e^{O(\sqrt{d \log d})}\right)$. A similar bound was obtained independently by Kalai 218] via a randomized pivot rule for the simplex algorithm. Clarkson's algorithm was subsequently derandomized; see [94] and the references therein for the latest developments.

6.5.3. Combinatorial abstractions of LP. Clarkson's algorithm uses very little structure from linear programming, namely the abilities to solve a small-size problem and to decide if a given solution violates a given constraint. Surprisingly, there are many computational problems for which these two operations can be performed effectively to find a solution and which are not linear. A simple example is the computation of the smallest enclosing circle of a finite point set $P \subset \mathbb{R}^{2}$. Here, the constraints are the points of $P$, the candidate solutions are the circles, and a circle violates a point if it does not enclose it. Observe that subsets of points that minimally define their enclosing centers have size at most 3, so in this case witness sets again have size at most 3 . It turns out that Clarkson's algorithm readily applies to this problem. This is not an LP in disguise: a generic instance may have witness sets of size 2 or 3.

Various combinatorial abstractions of LP were studied in order to understand precisely what class of problems can be solved with the randomized approach we described before. Consider an abstract set of constraints numbered from 1 to $\mathrm{m}$, and an objective function that associates to any set $S \subseteq[m]$ the value $f(S) \in \mathbb{R}$ of the optimum when only the constraints in $S$ are considered. A natural blackbox model allows us to compute $f(S)$ when $S$ has bounded size (independently of $m$ ), or to decide violations asking whether $f(S \cup\{i\}) \stackrel{?}{=} f(S)$. It turns out that Clarkson's algorithm can effectively compute $f(S)$ in this abstract model under three assumptions [264]:

(i) $f$ is decreasing under inclusion, that is, $f(S) \leq f(T)$ whenever $S \subseteq T \subseteq$ $[m]$;

(ii) $f$ is local in the sense that

$$
\begin{aligned}
& f(S) \neq f(S \cup\{i\}) \Leftrightarrow f(T) \neq f(T \cup\{i\}) \\
& \forall S \subseteq T \subseteq[m] \text { such that } f(S)=f(T), \text { and } \forall i \in[m] ; \text { and }
\end{aligned}
$$

(iii) witness sets have bounded size, where a witness set $S$ is a minimal subset of $[m]$ with $f(S)=f([m])$.

Functions $f$ satisfying (i) and (ii) are called LP-type problems or generalized linear programming problems. 
Any generic problem of the form (6.5) is LP-type; here by "generic" we mean that for every subset of constraints, $f$ achieves its minimum over the intersection of those constraints in only a bounded number of points. As noted in Proposition 6.6, controlling the size of the witness set for such problems, and thus the effectiveness of Clarkson's approach, is a matter of Helly-type theorems. Later a generalization, called violator spaces [182, was shown to give the precise family of problems solved by Clarkson's approach.

6.5.4. Chance-constrained optimization. Consider the problem of computing, given $n$ points in the plane, the smallest disk containing a given proportion of these points (say 70\%). More generally, given a probability measure $\mu$ in the plane and a positive number $\varepsilon$, one may consider the optimization problem

$$
\begin{array}{cl}
\min & r \\
\text { s.t. } & \operatorname{Pr}\left[\|\boldsymbol{x}-\boldsymbol{y}\|_{2} \leq r\right] \geq 1-\varepsilon, \\
& \boldsymbol{x} \in \mathbb{R}^{2},
\end{array}
$$

where $\boldsymbol{y}$ is a random point chosen from the probability distribution $\mu$. This quantitative variation of the smallest enclosing circle problem, discussed above, is no longer LP-type but can still be solved effectively. The technique relies, again, on the fact that witness sets have bounded size, and it applies more generally to a chance-constrained problem (CCP). A CCP asks to optimize a function of a variable $\boldsymbol{x} \in \mathbb{R}^{d}$ under constraints depending on a parameter $\boldsymbol{w} \in \Delta$ of the form:

$$
\begin{aligned}
C C P(\varepsilon)=\min & g(\boldsymbol{x}) \\
\text { s.t. } & \operatorname{Pr}(f(\boldsymbol{x}, \boldsymbol{w}) \leq 0) \geq 1-\varepsilon, \boldsymbol{x} \in K .
\end{aligned}
$$

Here, $g$ is a convex function, the probability is taken relative to a measure $\mu$ on the space $\Delta$ of parameters, $f(\boldsymbol{x}, \boldsymbol{w})$ is measurable with respect to $\boldsymbol{w}, f(\cdot, \boldsymbol{w})$ is convex for every $\boldsymbol{w}$, and $K$ is a convex set. This type of optimization problem naturally arises when modeling with uncertain constraints 351.

An approach to solve CCP, initiated by Calafiore and Campi [87, 88, is to sample $\boldsymbol{w}^{1}, \boldsymbol{w}^{2}, \ldots, \boldsymbol{w}^{N}$ from $\mu$ and solve the deterministic convex program

$$
\begin{aligned}
S C P(N)=\min & g(\boldsymbol{x}) \\
\text { s.t. } & f\left(\boldsymbol{x}, \boldsymbol{w}^{i}\right) \leq 0, \quad i=1,2, \ldots, N, \boldsymbol{x} \in K .
\end{aligned}
$$

For any $\delta \in(0,1)$, if $N \geq \frac{2 d}{\varepsilon} \ln \frac{1}{\varepsilon}+\frac{2}{\varepsilon} \ln \frac{1}{\delta}+2 d$, then the solution to $S C P(N)$ is a solution to $C C P(\varepsilon)$ with probability at least $1-\delta$ [87, 88.

The proof in [128] goes as follows. For $\boldsymbol{x} \in \mathbb{R}^{d}$, let $V(\boldsymbol{x})=\operatorname{Pr}(f(x, w)>0)$ so that we are interested in ensuring $V(\boldsymbol{x})<\varepsilon$. Each of the $N$ constraints $f\left(\cdot, \boldsymbol{w}^{i}\right) \leq 0$ is convex, so any witness set has size at most $d$. Now, for every $I \in\left(\begin{array}{c}{[N]} \\ d\end{array}\right)$, we define

$$
\Gamma_{N}^{I}=\left\{\left(\boldsymbol{w}^{1}, \ldots, \boldsymbol{w}^{N}\right) \in \Delta^{N}:\left(\boldsymbol{w}^{i}\right)_{i \in I} \text { is a witness set }\right\} .
$$

Note that the $\Gamma_{N}^{I}$ decompose $\Delta^{N}$ according to which ones are the indices of the $d$ witness constraints. Let $\boldsymbol{x}^{*}$ denote the optimal solution of $\operatorname{SCP}(N)$, and let $\boldsymbol{x}^{I}$ be the solution to the convex program defined by the constraints $\left\{\boldsymbol{w}^{i}: i \in I\right\}$ alone. The probability of failure $\operatorname{Pr}\left(V\left(\boldsymbol{x}^{*}\right) \geq \varepsilon\right)$ is less than or equal to

$$
\sum_{I \in\left(\begin{array}{c}
{[N]} \\
d
\end{array}\right)} \operatorname{Pr}\left(\left\{\left(\boldsymbol{w}^{1}, \ldots, \boldsymbol{w}^{N}\right) \in \Gamma_{N}^{I}: V\left(\boldsymbol{x}_{I}\right) \geq \varepsilon\right\}\right) .
$$


The summand corresponding to index set $I$ rewrites as

$$
\begin{aligned}
\operatorname{Pr} & {\left[\left\{\left(\boldsymbol{w}^{i}\right)_{i \in I}: V\left(\boldsymbol{x}_{I}\right) \geq \varepsilon\right\}\right] } \\
& \times \prod_{j \notin I} \operatorname{Pr}\left[\left\{\left(\boldsymbol{w}^{i}\right)_{i \in I}: f\left(\boldsymbol{x}_{I}, \boldsymbol{w}^{j}\right) \leq 0\right\} \mid\left\{\left(\boldsymbol{w}^{i}\right)_{i \in I}: V\left(\boldsymbol{x}_{I}\right) \geq \varepsilon\right\}\right] .
\end{aligned}
$$

The first factor is at most 1 and each of the following $N-d$ factors is at most $\varepsilon$. Altogether, we get $\operatorname{Pr}\left(V\left(\boldsymbol{x}^{*}\right)\right) \leq\left(\begin{array}{c}N \\ d\end{array}\right)(1-\varepsilon)^{N-d}$, and the announced bound follows.

6.5.5. S-optimization. There are many situations where one wants to optimize under convex constraints while restricting the solutions to belong to some set $S$; these are called $S$-optimization problems. This allows one to model complicated constraints such as mixed-integer constraints $\left(S=\mathbb{R}^{d} \times \mathbb{Z}^{k}\right)$, sparsity constraints (e.g., compressed sensing), or complementarity constraints.

Several of the techniques described above generalize if the intersections of $S$ with convex sets of the ambient space admits a Helly-type theorem. We denote its Helly number by $h(S)$, for example $h\left(\mathbb{R}^{d} \times \mathbb{Z}^{k}\right)=2^{k}(d+1)$. Proposition 6.6 yields that witness sets have size at most $h(S)-1$, and the reader can check that the analysis of chance constraint programs in Section 6.5.4 applies: the solution to $S C P(N)$ is a solution to $C C P(\varepsilon)$ with probability at least $1-\delta$ if

$$
N \geq \frac{2 h(S)-2}{\varepsilon} \ln \frac{1}{\varepsilon}+\frac{2}{\varepsilon} \ln \frac{1}{\delta}+2 h(S)-2 .
$$

In fact, the proof presented above differs from the initial argument 87, 88, and was found when generalizing CCP to the $S$-optimization setup [128. Helly-type theorems have been obtained for various sets $S$ [34, 129, 179, 319].

Let us now turn our attention to the problem of minimizing a convex function $g$ over an arbitrary subset $S \subseteq \mathbb{R}^{d}$. We assume that $g$ is given by a first-order evaluation oracle and that $S$ is nonempty and closed. The cutting plane method that we now present allows one to approximate the solution. To allow us to control the quality of the approximation, we fix a finite measure $\mu$ supported on $S$. The algorithm starts with a convex set $E_{0}$ that contains the solution in its interior and is such that $\mu\left(\operatorname{int} E_{0}\right)>0$. It then builds a sequence $\left\{E_{i}\right\}$ where each $E_{i}$ is also a convex set that contains the solution in its interior. Given $E_{i-1}$, we select a point $\boldsymbol{x}_{i} \in\left(\right.$ int $\left.E_{i-1}\right) \cap S$ and compute $g\left(\boldsymbol{x}_{i}\right)$ and a subgradient $\boldsymbol{h}_{i} \in \partial g\left(\boldsymbol{x}_{i}\right)$. We set $\boldsymbol{x}^{\star}:=\operatorname{argmin}_{\boldsymbol{x} \in\left\{\boldsymbol{x}_{1}, \ldots, \boldsymbol{x}_{i}\right\}} g(\boldsymbol{x})$ and define $E_{i}$ in a way that ensures that

$$
E_{i} \supseteq\left\{\boldsymbol{x} \in E_{0}: g\left(\boldsymbol{x}^{\star}\right)-g\left(\boldsymbol{x}_{j}\right) \geq \boldsymbol{h}_{j}^{T}\left(\boldsymbol{x}-\boldsymbol{x}_{j}\right) \quad \forall j \in[i]\right\},
$$

and that $\mu$ (int $\left.E_{i}\right)$ is nonincreasing. We stop when $\mu\left(\operatorname{int} E_{i}\right)$ is smaller than the desired error and return $\boldsymbol{x}^{*}$. This approach leaves many details unspecified, in particular the precise definition of $E_{i}$ and the way to choose the points $\boldsymbol{x}_{i}$. When $S=\mathbb{R}^{d}$ and $\mu$ is the Lebesgue measure, a possible implementation is the classical ellipsoid method [231. When $S=\mathbb{Z}^{d}$ and $\mu$ is the counting measure for $\mathbb{Z}^{d}$, we obtain cutting plane algorithms for convex integer optimization problems. Another variant of this method, which uses random sampling, was explored by Bertsimas and Vempala [59.

The choice of the points $\boldsymbol{x}_{i}$ in the cutting plane method is important. A particularly good choice are the Tukey centers. Given a vector $\boldsymbol{u} \in \mathbb{S}^{d-1}$ and a point 
$\boldsymbol{x} \in \mathbb{R}^{d}$, we let $H^{+}(\boldsymbol{u}, \boldsymbol{x})$ denote the halfspace $\left\{\boldsymbol{y} \in \mathbb{R}^{d}: \boldsymbol{u}^{T}(\boldsymbol{y}-\boldsymbol{x}) \geq 0\right\}$. Consider the function

$$
\mathcal{F}(S, \mu):=\max _{\boldsymbol{x} \in S} \inf _{\boldsymbol{u} \in \mathbb{S}^{n-1}} \mu\left(H^{+}(\boldsymbol{u}, \boldsymbol{x})\right) .
$$

A Tukey center is a point that attains the maximum value of $\mathcal{F}(S, \mu)$. Lower bounds on $\mathcal{F}(S, \mu)$, and therefore on the depth of Tukey centers (see Section 7.4), can often be obtained from Helly-type theorems. For instance, if $S=\mathbb{R}^{d}$ and $\mu$ is the counting measure of a finite subset of $\mathbb{R}^{d}$, then the centerpoint theorem (Theorem 7.12 ) ensures that $\mathcal{F}(S, \mu) \geq \frac{1}{d+1}|P|$. The proof of the centerpoint theorem from Helly's theorem extends to the setting of $S$-convexity: if $S \subseteq \mathbb{R}^{d}$ is nonempty and closed and $\mu$ is finite and supported on $S$, then $\mathcal{F}(S, \mu) \geq \frac{1}{h(S)} \mu\left(\mathbb{R}^{d}\right)$ [55. For instance, Doignon's theorem ensures that if $S=\mathbb{Z}^{d}$ and $\mu_{C}$ counts integer points inside a compact set $C$, then $\mathcal{F}\left(S, \mu_{C}\right) \geq \frac{\left|C \cap \mathbb{Z}^{d}\right|}{2^{d}}$. It turns out that choosing $\boldsymbol{x}_{i}$ among the points maximizing $\mathcal{F}\left(\mu_{i}, S\right)$, where $\mu_{i}$ is the restriction of $\mu$ to $\operatorname{int}\left(E_{i-1}\right)$, gives the best running times among cutting plane algorithms for convex minimization over $S$ [55.

More notions generalize to $S$-optimization. For instance there exists an analogue of the strong duality theorem for $S$-convex optimization under some natural conditions [54. We will meet Tukey centers again in Section 7.4] and we conclude this section with a challenge:

Open Problem 6.8. What is the complexity of computing Tukey centers for given $S$ and $\mu$ ? For example, can one compute an exact Tukey center of the integer points of a convex polytope in polynomial time in the input size?

\section{DAta POINT SETS}

In this section we consider some computer science results that are either applications of the main theorems or strongly related to them. There are simply far too many results for us to do justice to even a small number of them, thus we restrict ourselves to a few central themes, including classification, geometric shape analysis, and partitioning of $n$ points in $d$-dimensional Euclidean spaces. These will involve an interplay between affine geometric and topological techniques, offering the usual mix of advantages and drawbacks of the two: Affine tools - Radon's lemma, Helly's theorem, linear programming duality, simplicial decompositions - will imply fast algorithms, though they apply to a restricted group of geometric objects. Topological tools - the Borsuk-Ulam theorem, Tucker's lemma-yield broader structural statements, though, at this moment, settling the algorithmic feasibility of these methods remains a major open problem.

7.1. Equipartitioning: the ham sandwich theorem and its relatives. We say that a hyperplane $h$ bisects a set $P$ of points if the two open halfspaces defined by $h$ contain at most $\frac{|P|}{2}$ points of $P$. Note that if $|P|$ is odd, then a point of $P$ must necessarily lie on $h$. The famous ham sandwich theorem is the starting point of a large number of results concerning equipartitioning of geometric objects with other geometric objects.

Theorem 7.1. Let $P_{1}, \ldots, P_{d}$ be finite point sets in $\mathbb{R}^{d}$. Then there exists a hyperplane $h$ that simultaneously bisects each $P_{i}, i=1, \ldots, d$. 
The theorem holds more generally for finite Borel measures that evaluate to zero on every affine hyperplane. All known proofs of Theorem 7.1 are essentially topological in nature. A classical proof follows from the Borsuk-Ulam theorem by identifying points of $\mathbb{S}^{d}$ with hyperplanes in $\mathbb{R}^{d}$, and where the function $f: \mathbb{S}^{d} \rightarrow \mathbb{R}^{d}$ encodes the imbalance of the $d$ point sets (more generally, measures) with respect to that hyperplane [258. We now outline another proof of the ham sandwich theorem using Tucker's lemma. The proof we present was found independently by Holmsen and by the third author (both unpublished).

For simplicity we will assume that the given point sets $P_{1}, \ldots, P_{d}$ are in general position, and let $\bigcup_{i=1}^{d} P_{i}=\left\{\boldsymbol{p}_{1}, \ldots, \boldsymbol{p}_{n}\right\}$. Each pair $(\boldsymbol{a}, b) \in\left(\mathbb{R}^{d} \times \mathbb{R}_{+}\right) \backslash\{\mathbf{0}, 0\}$ induces a sign pattern $\boldsymbol{x} \in\{+,-, 0\}^{n}$ with $x_{j}$ being the sign of $\boldsymbol{a} \cdot p_{j}+b$. We will apply Tucker's lemma (Proposition 2.2) on an abstract simplicial complex induced by these sign patterns to show that there is a pair $(\boldsymbol{a}, b)$ whose sign pattern $\boldsymbol{x}$ is such that $\left\{p_{j}: j \in \boldsymbol{x}^{+}\right\}$and $\left\{p_{j}: j \in \boldsymbol{x}^{-}\right\}$each contain at most half of each $P_{i}$. Then the hyperplane $\left\{\boldsymbol{y} \in \mathbb{R}^{d}: \boldsymbol{a} \cdot \boldsymbol{y}+b=0\right\}$ bisects each $P_{i}$.

Denote by $\mathcal{P}$ the partially ordered set of all achievable sign patterns endowed with the partial order $\preceq$ (see Section 2 before Tucker's octahedral lemma). It is a well-known result from oriented matroid theory that the order complex $T$ of $\mathcal{P}$ is a triangulation of $\mathbb{B}^{d}$ that is symmetric on its boundary. Suppose, for contradiction, that there is no hyperplane bisecting each $P_{i}$. Given an $\boldsymbol{x}$ in $\mathcal{P}$, we define $\lambda(\boldsymbol{x})$ to be $\varepsilon i$, where $i$ is the smallest index such that either $\left\{\boldsymbol{p}_{j}: j \in \boldsymbol{x}^{+}\right\} \cap P_{i}$ or $\left\{\boldsymbol{p}_{j}: j \in \boldsymbol{x}^{-}\right\} \cap P_{i}$ contains more than half of the points of $P_{i}$, and where $\varepsilon$ is + if it is the first set and is - if it is the second. This map $\lambda$ is clearly antipodal on the boundary of $\mathrm{T}$ and labels the vertices of $\mathrm{T}$ with the elements of $\{ \pm 1, \ldots, \pm d\}$. According to Tucker's lemma, there exists an edge $\boldsymbol{u} \boldsymbol{v}$ of T with $\lambda(\boldsymbol{u})+\lambda(\boldsymbol{v})=0$. Without loss of generality, we assume that $\boldsymbol{u} \preceq \boldsymbol{v}$ and that $-\lambda(\boldsymbol{u})=\lambda(\boldsymbol{v})=k$ for some $k \in[d]$. By definition of $\lambda$, we have then $\left|\left\{\boldsymbol{p}_{j}: j \in \boldsymbol{u}^{-}\right\} \cap P_{k}\right|>\left|P_{k}\right| / 2$ and $\left|\left\{\boldsymbol{p}_{j}: j \in \boldsymbol{v}^{+}\right\} \cap P_{k}\right|>\left|P_{k}\right| / 2$. Combined with $\boldsymbol{u}^{-} \subseteq \boldsymbol{v}^{-}$, it implies that $\left|\left\{\boldsymbol{p}_{j}: j \in \boldsymbol{v}^{-} \cup \boldsymbol{v}^{+}\right\} \cap P_{k}\right|>\left|P_{k}\right|$, a contradiction.

We will see further applications of the ham sandwich theorem later on, but for now we point out that it gives another proof of Theorem 4.9, given an open necklace with $t$ types of beads to be divided equally between two thieves, embed the beads of the necklace along a moment curve in $\mathbb{R}^{t}$, and use a hyperplane $h$ guaranteed by Theorem 7.1 to bisect each type of bead. As any hyperplane intersects a moment curve at $t$ points, $h$ splits the open necklace into $t+1$ pieces that can then be divided among the two thieves.

Note that from the above discussion, we have the following computational hierarchy: computing a solution to this variation of the octahedral Tucker problem is harder than computing a ham sandwich cut (note also that this implies that the latter is in PPA), which is harder than computing a solution to the fair splitting necklace problem. In particular, the Filos-Ratsikas and Goldberg paper [164] proves that computing the ham sandwich cut is PPA-complete (see Section 4.2).

As far as the efficiency aspects of Theorem 7.1 are concerned, given point sets, of a total of $n$ points in $\mathbb{R}^{2}$, a bisecting line can be computed in $O(n)$ time. In $\mathbb{R}^{d}$ the best algorithm for computing a ham sandwich cut for $d$ point sets in $\mathbb{R}^{d}$ runs in time $O\left(n^{d-1}\right)$ [245]; in fact the algorithm proposed presents a new proof of Theorem 7.1] that proceeds by induction on the dimension and thus is more amenable to efficient algorithm design. 
By now there are dozens of variants of the ham sandwich theorem, and generalizations to other types of bisecting and bisected objects. We now present a few nice examples. For the ham sandwich theorem, see also [258, 398] and references therein.

One variation is called the center transversal theorem: given $s+1$ point sets in $\mathbb{R}^{d}$ where $s \in\{0, \ldots, d-1\}$, there exists an $s$-dimensional affine subspace $h$ of $\mathbb{R}^{d}$ such that any hyperplane containing $h$ has at least $\frac{1}{(d-s+1)}$-th fraction of the points of $P_{i}$ on each side, for $i=0, \ldots, s+1$. In fact, it has been conjectured that the constant $\frac{1}{(d-s+1)}$ can be replaced by $\frac{(s+1)}{(d+s+1)}$; see 86. Theorem 7.1 is the case $s=d-1$, and the case $s=0$ is the important centerpoint theorem that we encountered in the introduction and will visit again later. We refer the reader to the book 258. for many variants of this and related theorems in $\mathbb{R}^{2}$ and higher dimensions. Here now are two famous conjectures.

Conjecture 7.2. Let $P$ be a set of points in $\mathbb{R}^{4}$. Then there exists a set $\mathcal{H}$ of four hyperplanes such that each of the resulting 16 open regions of $\mathbb{R}^{4} \backslash \mathcal{H}$ contains at most $\frac{|P|}{16}$ points of $P$.

In the papers 64,65 , the authors showed that in $\mathbb{R}^{5}$ it is indeed possible to find four hyperplanes that divide the set into 16 equal parts; see also [354. We should mention here a related theorem of Yao and Yao 393] (see also Theorem[7.8): Given a set $P$ of $n$ points in $\mathbb{R}^{d}$, one can partition $\mathbb{R}^{d}$ into $2^{d}$ regions such that the interior of each region contains at most $\frac{n}{2^{d}}$ points of $P$, and any hyperplane intersects the interior of at most $2^{d}-1$ regions.

Next we consider another conjecture by Tverberg and Vrećica 378.

Conjecture 7.3. Let $0 \leq k \leq d-1$ be a given parameter, and let $P_{0}, P_{1}, \ldots, P_{k}$ be finite point sets in $\mathbb{R}^{d}$. If $\left|P_{i}\right|=\left(r_{i}-1\right)(d-k+1)+1$ for $i=0,1, \ldots, k$, then each $P_{i}$ can be partitioned into $r_{i}$ parts $P_{i, 1}, P_{i, 2}, \ldots, P_{i, r_{i}}$ such that the sets $\left\{\operatorname{conv}\left(P_{i, j}\right): 0 \leq i \leq k, 1 \leq j \leq r_{i}\right\}$ can be intersected by a $k$-dimensional affine space.

Note that Tverberg's theorem is the case when $k=0$ in the above statement.

If one wants to partition more than $d$ point sets in $\mathbb{R}^{d}$, then hyperplanes are often insufficient; however the following important variant of the ham sandwich theorem, due to Stone and Tukey [365], shows that then polynomials of a sufficiently high degree can be used to do the partition.

We say that a $d$-variate polynomial $f \in \mathbb{R}\left[x_{1}, \ldots, x_{d}\right]$ bisects a point set $P \subseteq \mathbb{R}^{d}$ if it evaluates to negative on at most $\frac{|P|}{2}$ points of $P$ and likewise evaluates to positive on at most $\frac{|P|}{2}$ points of $P$. Note that for the case of polynomials of degree 1 , this coincides with our earlier definition of bisection for hyperplanes.

Theorem 7.4. Let $P_{1}, \ldots, P_{s}$ be finite point sets in $\mathbb{R}^{d}$. Then there exists a $d$-variate polynomial $f \in \mathbb{R}\left[x_{1}, \ldots, x_{d}\right]$ of degree $O\left(s^{\frac{1}{d}}\right)$ such that $f$ bisects each $P_{i}, i=1, \ldots, s$.

The idea is to reduce the above problem to the usual ham sandwich theorem in a suitably high dimension. As a $d$-variate polynomial of degree $D$ has $d^{\prime}=\left(\begin{array}{c}D+d \\ d\end{array}\right)-1$ monomials (aside from the constant term), identify each such monomial with a distinct dimension of $\mathbb{R}^{d^{\prime}}$. Then each $d$-variate polynomial $f$ of degree $D$ can be identified with a hyperplane in $\mathbb{R}^{d^{\prime}}$, where the coefficients defining the hyperplane 
in $\mathbb{R}^{d^{\prime}}$ (i.e., the $d^{\prime}$ coordinates of the normal vector of the hyperplane) correspond to the coefficients of the corresponding monomials of $f$. This also gives a mappingcalled a Veronese mapping - of the points in $P_{1} \cup \cdots \cup P_{s}$ to $\mathbb{R}^{d^{\prime}}$, where the $i$ th coordinate of a point $p \in \mathbb{R}^{d}$ is the value of the corresponding monomial on $p$. One can now use Theorem 7.1 on the $d$ sets corresponding to the lifted points of $P_{1}, \ldots, P_{s}$ to get a hyperplane $h$ in $\mathbb{R}^{d^{\prime}}$ that bisects each of the $s$ lifted sets. Note that to use the ham sandwich theorem, we require $s \leq d^{\prime}=\left(\begin{array}{c}D+d \\ d\end{array}\right)-1$ and thus need to satisfy the constraint $D=\Omega\left(s^{\frac{1}{d}}\right)$. The ham sandwich hyperplane $h$ corresponds to the required $d$-variate polynomial in $\mathbb{R}^{d}$, of degree $O\left(s^{\frac{1}{d}}\right)$.

7.2. Parametrized partitioning of data via geometric methods. So far the partitioning statements have been of the type where the input geometric configuration precisely fixes the output type; e.g., given $d$ point sets in $\mathbb{R}^{d}$ fixes the output of Theorem 7.1 to be a hyperplane, or given $s$ point sets in $\mathbb{R}^{d}$ in Theorem 7.4 fixes the output to be a polynomial of degree $O\left(s^{\frac{1}{d}}\right)$. Now we consider statements where, besides the input geometric configuration, one is also given an independent parameter $r$ and the complexity of the output is a function of both the geometric configuration as well as the value of $r$. Thus one gets a hierarchy of output structures (varying with $r$ ), and one is free to choose the value of $r$ depending on the precise problem at hand. This turns out to be very useful for designing hierarchical data structures where one can pick the value of $r$ to maximize computational efficiency.

These kinds of partitioning statements, which we call parameterized spatial partitioning, have been a key theme in discrete and computational geometry for both algorithmic and proof purposes. Consider, for example, Hopcroft's problem studied in the early 1980s: Given a set of lines $L$ and a set of points $P$, an incidence is a pair $(\boldsymbol{p}, l)$, where $\boldsymbol{p} \in P, l \in L$, and the point $\boldsymbol{p}$ lies on the line $l$. Then given $L$ and $P$, is there an efficient method to determine if there exists at least one incidence between them? It is not difficult to see that a spatial partition that either partitions the points or partitions the line (in a suitable sense) is useful for decomposing the original problem into several problems of smaller size. The current best algorithm with a running time of $2^{O\left(\log ^{*} n\right)} \cdot n^{\frac{4}{3}}$ is based on such techniques. We refer the reader to [157] for details on this and other related results on Hopcroft's problem.

For a more mathematical application in a similar setting, consider the following question posed by Erdös [156]: What is the maximum number of incidences between any $n$ points and any $n$ lines in the plane? Erdös observed that as the bipartite incidence graph between points and lines is $K_{2,2}$-free, this is upper-bounded by $O\left(n^{\frac{3}{2}}\right)$. More generally, the maximum number of incidences between $m$ lines and $n$ points is $O(n \sqrt{m})$. On the other hand, a set of points in a "grid-like" configuration exhibits $\Omega\left(n^{\frac{4}{3}}\right)$ incidences, and Erdös conjectured this to be, asymptotically, the right bound.

This question was resolved affirmatively by Szemerédi and Trotter by a complicated combinatorial argument [371]. We sketch here a beautiful and simple proof of this theorem by Clarkson et al. [11] that showcases the use of spatial partitioning for proving combinatorial bounds. Given a set $L$ of $n$ lines in the plane, suppose that for any parameter $r>1$, there exists a partition of the plane into $t=O\left(r^{2}\right)$ (possibly unbounded) interior-disjoint triangles $\Pi=\left\{\triangle_{1}, \ldots, \triangle_{t}\right\}$ such that each triangle $\triangle_{i}$, for $i=1 \cdots t$, intersects at most $\frac{n}{r}$ lines of $L$ in its interior. Now one 
can partition the incidences between $P$ and $L$ into those for which the points lie on boundary of some triangle, and those for which the points lie in the interior of some triangle of $\Pi$. It is not difficult to see that the former can be only $O(n r)$; on the other hand, a triangle $\triangle_{i}$ intersecting $m_{i}$ lines of $L$ in its interior and containing $n_{i}$ points of $P$ can contain $O\left(n_{i} \sqrt{m_{i}}\right)$ incidences in its interior (via the graph-theoretic bound). This gives the overall number of incidences lying in the interior of triangles to be $\sum_{i} O\left(n_{i} \sqrt{m_{i}}\right)=O\left(n \sqrt{\frac{n}{r}}\right)$. Thus for any $r>1$, the total number of incidences is bounded by $O\left(n r+\frac{n^{\frac{3}{2}}}{\sqrt{r}}\right)$, and setting $r=\Theta\left(n^{\frac{1}{3}}\right)$ gives the desired bound!

We now elaborate on this structural partitioning problem and its variations. The key behind the proof is the partition of $\mathbb{R}^{2}$ into triangles, each of which intersects proportionally few lines of $L$. More generally, in any dimension, one can show the existence of a similar partition of hyperplanes [96].

Theorem 7.5. Given a set $\mathcal{H}$ of hyperplanes in $\mathbb{R}^{d}$ and a parameter $r \geq 1$, there exists a partition of $\mathbb{R}^{d}$ into $\Theta\left(r^{d}\right)$ interior-disjoint simplices such that the interior of any simplex intersects at most $\frac{|\mathcal{H}|}{r}$ hyperplanes of $\mathcal{H}$.

Such a partition is called a $\frac{1}{r}$-cutting of $\mathcal{H}$. Note that the bound of $\Theta\left(r^{d}\right)$ cannot be improved: each simplex can contain at most $\left(\frac{|\mathcal{H}|}{r}\right)^{d}$ vertices induced by $d$-tuples of $\mathcal{H}$ in its interior, and so there must be $\Omega\left(r^{d}\right)$ simplices to account for all the $\Theta\left(|\mathcal{H}|^{d}\right)$ vertices induced by $\mathcal{H}$.

The intuition behind Theorem 7.5 is as follows. Pick each hyperplane of $\mathcal{H}$ into a random sample $R$ independently with probability $p$ (to be set later). Then $\mathrm{E}[|R|]=|\mathcal{H}| \cdot p$, and so $R$ partitions $\mathbb{R}^{d}$ into $O\left((|\mathcal{H}| p)^{d}\right)$ induced cells, each of which can then be further partitioned into simplices, to get a partition of $\mathbb{R}^{d}$ into an expected total of $O\left((|\mathcal{H}| p)^{d}\right)$ simplices. Furthermore, note that each such simplex intersects, in expectation, $\frac{1}{p}$ hyperplanes of $\mathcal{H}$ in its interior. Setting $p=\frac{r}{|\mathcal{H}|}$ gives the required statement. This argument was done "in expectation", and it is nontrivial to convert it with the same asymptotic bounds to where each simplex is guaranteed to intersect no more than $\frac{|\mathcal{H}|}{r}$ hyperplanes of $\mathcal{H}$.

The proof of Theorem 7.5 is usually presented in the more general abstract framework of the theory of $\varepsilon$-nets, whose setting we briefly describe now. Given a base set of elements $X$, a set system $\mathcal{R}$ on $X$, and a parameter $0 \leq \varepsilon \leq 1$, call a set $N \subseteq X$ an $\varepsilon$-net for $\mathcal{R}$ if $N$ contains at least one element from each $R \in \mathcal{R}$ with $|R| \geq \varepsilon \cdot|X|$. In the case of $\frac{1}{r}$-cuttings, the set $X$ is the set of hyperplanes $\mathcal{H}$, and $R \in \mathcal{R}$ if and only if there exists a simplex $\Delta$ with $R=\operatorname{int}(\Delta) \cap \mathcal{H}$. Then a $\frac{1}{r}$ cutting can be constructed by taking an $\varepsilon$-net $N$ for $\mathcal{R}$ with $\varepsilon=\frac{1}{r}$ and partitioning $\mathbb{R}^{d}$ into $O\left(|N|^{d}\right)$ simplices using $N$. As the interior of each simplex induced by $N$ does not intersect any hyperplane of $N$, it can only intersect less than $\varepsilon \cdot|\mathcal{H}|=\frac{|\mathcal{H}|}{r}$ hyperplanes of $\mathcal{H}$, as desired.

Bounds on $\varepsilon$-nets have been extensively studied for set systems satisfying a combinatorial condition, called the Vapnik-Chervonenkis (VC) dimension [383]: Given a set system $(X, \mathcal{R})$, define the projection of $\mathcal{R}$ onto a set $Y \subseteq X$, denoted $\left.\mathcal{R}\right|_{Y}$, as the set system $\left.\mathcal{R}\right|_{Y}=\{Y \cap R: R \in \mathcal{R}\}$. We say that a subset $Y$ is shattered by $\mathcal{R}$ if all $2^{|Y|}$ subsets of $Y$ can be realized by intersection with some set of $\mathcal{R}$, i.e., if $|\mathcal{R}|_{Y} \mid=2^{|Y|}$. Then the VC dimension of $\mathcal{R}$ is defined to be the size of the largest set that is shattered by $\mathcal{R}$. 
The VC dimension plays an important role in the theory of set systems derived from geometric configurations due to the fact that the VC dimension of such systems is usually quite small. For example, consider the set system where $X$ is a finite set of $n$ points in $\mathbb{R}^{d}$, and the subsets in $\mathcal{R}$ are derived from intersection with halfspaces; here $R \in \mathcal{R}$ if and only if there exists a halfspace $h$ such that $R=h \cap X$. The VC dimension of this set system is $d+1$; in other words, given any set $X$ of $d+2$ points in $\mathbb{R}^{d}$, one cannot "separate" all subsets of $X$ by intersection with halfspaces. This is an immediate consequence of Radon's lemma (recall that Radon's lemma is the case of Tverberg's theorem for two parts, $r=2$, namely that any set $P$ of $d+2$ points in $\mathbb{R}^{d}$ can be partitioned into two subsets $P_{1}, P_{2}$ such that $\left.\operatorname{conv}\left(P_{1}\right) \cap \operatorname{conv}\left(P_{2}\right) \neq \varnothing\right)$ : the convex hulls of the Radon partitions intersect, and thus cannot be separated by a hyperplane. On the other hand, any set of $d+1$ points in general position can be shattered by halfspaces in $\mathbb{R}^{d}$. By Veronese maps, this implies more generally bounded VC dimension for set systems induced by geometric objects of bounded algebraic complexity (see [257, Chapter 10]).

Returning to $\varepsilon$-nets, building on the work of Vapnik and Chervonenkis 383 , Haussler and Welzl 203. showed the existence of small $\varepsilon$-nets as a function of the $\mathrm{VC}$ dimension of a set system.

Theorem 7.6. Let $(X, \mathcal{R})$ be a finite set system with $V C$ dimension at most $d \geq 1$. Then for any real parameter $\varepsilon>0$, there exists an $\varepsilon$-net for $\mathcal{R}$ of size $O\left(\frac{d}{\varepsilon} \log \frac{1}{\varepsilon}\right)$.

The power of this theorem derives from the fact that the size is independent of the number of elements in $X$ and the number of sets in $\mathcal{R}$. Combined with the VC dimension bound of $d+1$ on set systems induced on points in $\mathbb{R}^{d}$ by halfspaces, Theorem 7.6 implies the existence of $\varepsilon$-nets of size $O\left(\frac{d}{\varepsilon} \log \frac{1}{\varepsilon}\right)$, which has been shown to be optimal 239. The bounds of Theorem 7.6 can be further improved for many geometric set systems, and recent work presents a unified framework for these bounds [95, 292, 384. We refer the reader to the books [311, Chapter 15], [257, Chapter 10], [97, Chapter 4], and [256, Chapter 5] for more detailed expositions on $\varepsilon$-nets and their many applications.

The theory of $\mathrm{VC}$ dimension fails to help in the construction of $\varepsilon$-nets when it is unbounded. A basic case is the set system induced by convex objects in $\mathbb{R}^{d}$; namely, given a set $P$ of $n$ points in $\mathbb{R}^{d}$ and a parameter $\varepsilon>0$, one would like to show the existence of a small set $Q \subset \mathbb{R}^{d}$, called a weak $\varepsilon$-net for $P$ induced by convex objects, such that any convex object containing at least $\varepsilon n$ points of $P$ must contain at least one point of $Q$. Note here that $Q$ can be any set of points in $\mathbb{R}^{d}$, and is not just limited to being a subset of $P$-hence the term weak.

An initial bound of $O\left(\frac{1}{\varepsilon^{d+1}}\right)$ on the size of $Q$ was shown by Alon et al. 20] (their proof uses the colorful Carathéodory's theorem together with Tverberg's theorem; see the discussion about simplicial depth in Section 7.4), and this was improved to $\tilde{O}\left(\frac{1}{\varepsilon^{d}}\right)$ by Chazelle et al. [98. This was improved even further, by logarithmic factors, by Matoušek and Wagner [265] whose elegant proof we outline now. Partition $P$ into $t$ equal sized subsets $\mathcal{P}=\left\{P_{1}, \ldots, P_{t}\right\}$, for a parameter $t$ that is chosen optimally, such that any hyperplane intersects the convex hulls of $O\left(t^{1-1 / d}\right)$ subsets of $\mathcal{P}$. Let $C$ be a convex object containing $\varepsilon$ points of $P$. When $C$ intersects few sets of $\mathcal{P}$, the proportion of points of $C$ is contained in some $P_{i} \in \mathcal{P}$ is higher than $\varepsilon$, hence $C$ can be hit by a set constructed inductively for $P_{i}$. Otherwise, $C$ intersects many sets of $\mathcal{P}$. In this case, pick one arbitrary point from each set of $\mathcal{P}$ intersecting $C$. Let $\boldsymbol{q}$ be the centerpoint of those points. Then 
$\boldsymbol{q}$ must be contained in $C$, as otherwise the hyperplane separating $\boldsymbol{q}$ from $C$ must intersect the convex hulls of many sets in $\mathcal{P}$, a contradiction to the definition of $\mathcal{P}$.

For the case $d=2$, Rubin 332] proved the bound of $O\left(\frac{1}{\varepsilon^{1.5+\delta}}\right)$, where $\delta>0$ is an arbitrarily small constant.

Finding asymptotically optimal bounds for weak $\varepsilon$-nets induced by convex objects in $\mathbb{R}^{d}$ is a tantalizing open problem. The best known lower bounds of $\Omega\left(\frac{1}{\varepsilon}\left(\log \frac{1}{\varepsilon}\right)^{d}\right)$ [85] are quite far from the upper bounds. On the other hand, there are partial results that indicate the upper bounds that can be improved. For example, it is known 293] that one can construct weak $\varepsilon$-nets from $\tilde{O}\left(\frac{1}{\varepsilon}\right)$ points of $P$ in $\mathbb{R}^{d}$. Pick a set $Q^{\prime}$ of $\tilde{O}\left(\frac{1}{\varepsilon}\right)$ points that form an $\varepsilon$-net for the set system induced by the intersection of $d$ halfspaces. Then adding points lying in Tverberg partitions of carefully chosen subsets of $Q^{\prime}$ results in a weak $\varepsilon$-net, though of size $O\left(\frac{1}{\varepsilon^{d+2}}\right)$. For a different formulation, one can also fix an integer parameter $k>0$, and then ask for the minimization problem. Find $\varepsilon=\varepsilon(k)$ such that for any set $P$ of points in $\mathbb{R}^{d}$ there exists a set $Q \subset \mathbb{R}^{d}$ of $k$ points such that all convex objects containing at least $\varepsilon \cdot|P|$ points of $P$ are hit by $Q$; see [294].

Open Problem 7.7. What is the asymptotically best bound for the size of the smallest weak $\varepsilon$-net for the set system induced by convex objects in $\mathbb{R}^{d}$ ?

We next turn to the algorithmic aspects of spatial partitioning. There has been substantial work on improving the constants in the bounds on $\varepsilon$-nets, as they are directly linked to the approximation ratios of algorithms for the geometric hitting set problem [81,159]. Given a set $P$ of points in $\mathbb{R}^{d}$ and a set $\mathcal{O}$ of geometric objects, the goal is to compute a minimum subset of $P$ that hits all the objects in $\mathcal{O}$. This can be written as an integer program, which is then approximated as follows:

(a) solve the linear relaxation of the integer program (i.e., the linear program obtained by replacing the integral constraints with real ones); and

(b) assign weights to the points of $P$ according to the linear program; and finally

(c) compute a $\frac{1}{W}$-net $N$ for the set system induced by $\mathcal{O}$, where $W$ is the value of the linear programming relaxation.

As the weight of the points contained in each object of $\mathcal{O}$ is at least 1 by the linear program, $N$ is a hitting set for $\mathcal{O}$. The size of $N$ is then bounded by the size of a $\frac{1}{W}$-net; e.g., if an $\varepsilon$-net exists of size $\frac{c}{\varepsilon}$ for some constant $c$, then $N$ has size at most $c \cdot W$; i.e., at most $c$ times the optimal solution. We refer the reader to the recent surveys [105, 299] for precise bounds on $\varepsilon$-nets and cuttings for various geometric set systems as well as the current best algorithms for computing such nets.

Cuttings, together with linear programming duality (or alternatively, Farkas's lemma), can be used to derive another important partitioning tool we have already encountered in the construction of weak $\varepsilon$-nets - the simplicial partition theorem 255.

Theorem 7.8. Let $P$ be a set of points in $\mathbb{R}^{d}$, and let $t>1$ be a given integer parameter. Then there exists a partition of $P$ into $t$ sets $\mathcal{P}=\left\{P_{1}, \ldots, P_{t}\right\}$ such that $(a)\left|P_{i}\right| \leq \frac{2|P|}{t}$ for all $i=1, \ldots, t$ and $(b)$ any hyperplane intersects the convex hull of $O\left(t^{1-\frac{1}{d}}\right)$ sets of $\mathcal{P}$. 
We outline the general idea of the proof. Given $P$, one can first discretize the space of all possible hyperplanes in $\mathbb{R}^{d}$ into a finite set $\mathcal{H}$ of hyperplanes, so that one only needs to construct a partition $\mathcal{P}$ such that each hyperplane of $\mathcal{H}$ intersects the convex hulls of $O\left(t^{1-\frac{1}{d}}\right)$ sets of $\mathcal{P}$. Construct a $\Theta\left(\frac{1}{t^{1 / d}}\right)$-cutting for $\mathcal{H}$, consisting of at most $t$ simplices, and let $\mathcal{P}^{\prime}$ be the collection of points of $P$ lying in each simplex of the cutting. Then, on average, $\left|P^{\prime}\right|=\Theta\left(\frac{|P|}{t}\right)$ for each $P^{\prime} \in \mathcal{P}^{\prime}$, and furthermore the convex hull of each $P^{\prime}$ is intersected by at most $\frac{|\mathcal{H}|}{t^{1 / d}}$ hyperplanes of $\mathcal{H}$. This is, in a suitable sense, the dual of the statement that we want, where each hyperplane should intersect few cells in the cutting (and thus few convex hulls of sets in $\mathcal{P}^{\prime}$ ). However we are not far off-the total number of intersecting pairs of hyperplanes of $\mathcal{H}$ with convex hulls of sets in $\mathcal{P}^{\prime}$ is $O\left(t \cdot \frac{|\mathcal{H}|}{t^{1 / d}}\right)=O\left(t^{1-\frac{1}{d}} \cdot \mathcal{H}\right)$. In other words, the average hyperplane in $\mathcal{H}$ intersects the convex hulls of $O\left(t^{1-\frac{1}{d}}\right)$ sets of $\mathcal{P}^{\prime}$. Now LP duality [202] (or Farkas's lemma [183]) shows the existence of the required partition.

7.3. Parametrized partitioning of data via topological methods. Now we move to more recent approaches to parameterized spatial partitioning. These methods use equipartitioning results such as the ham sandwich theorem and Theorem 7.4 to show the existence of polynomials that induce parametrized partitions of $\mathbb{R}^{d}$. The resulting statements are similar in spirit and use those we saw earlier for affine objects. The main advantage of these newer approaches is that partitioning space with polynomials circumvents several difficult technical issues that arise when dealing with piecewise linear objects. On the other hand, new computational challenges arise in the topological approaches, as they often do not lend themselves to efficient algorithm design.

We present now a polynomial version of Theorem 7.8, discovered by Guth and Katz [198. For more on the impact of this theorem, see the book [197]. In what follows given a polynomial $f$, we denote by $Z(f)$ the zero set of $f$.

Theorem 7.9. Let $P$ be a set of $n$ points in $\mathbb{R}^{d}$, and let $r>1$ be a given parameter. Then there exists a d-variate polynomial $f$ of degree $O\left(r^{\frac{1}{d}}\right)$ such that each connected component of $\mathbb{R}^{d} \backslash Z(f)$ contains at most $\frac{|P|}{r}$ points.

Here the points lying in the components of $\mathbb{R}^{d} \backslash Z(f)$ play the role of the sets in Theorem 7.8 . Observe that any hyperplane $h$ in $\mathbb{R}^{d}$ intersects $O\left((\operatorname{deg}(f))^{d-1}\right)=$ $O\left(r^{1-\frac{1}{d}}\right)$ different components of $\mathbb{R}^{d} \backslash Z(f)$, quantitatively the same bound as in Theorem 7.8. In contrast with Theorem 7.8, these components are interior disjoint, though they will, in general, not be convex.

We sketch a proof: Partition $P$ into two equal sized sets $P_{1}, P_{2}$ by a polynomial, say $f_{0}$, of degree $O(1)$ (using Theorem 7.1 for example). Then partition these two point sets $P_{1}$ and $P_{2}$ into four equal disjoint subsets $P_{3}, P_{4}, P_{5}, P_{6}$ using a polynomial, say $f_{1}$, of degree $O\left(2^{\frac{1}{d}}\right)$ via Theorem 7.4. Continuing, let $f_{i}$ be a polynomial of degree at most $O\left(\left(2^{i}\right)^{\frac{1}{d}}\right)$ that equipartitions $2^{i}$ equal-sized disjoint subsets of $P$, for $i=0, \ldots, \log r$. Note here that as long as $2^{i} \leq d$, a hyperplane suffices for $f_{i}$. The key observation now is that the polynomial $f$ formed by taking the product of all these polynomials, namely $f=\prod_{i=0}^{\log r} f_{i}$, is the required polynomial: as the zero set of $f$ is simply the union of the zero sets of all the $f_{i}$ 's, each connected region of $\mathbb{R}^{d} \backslash Z(f)$ can contain at most $\frac{|P|}{2^{\log r}}=\frac{|P|}{r}$ points of $P$. The degree of $f$ can be 
bounded as

$$
\operatorname{deg}(f) \leq \sum_{i=0}^{\log r} \operatorname{deg}\left(f_{i}\right) \leq \sum_{i=0}^{\log r} O\left(\left(2^{i}\right)^{\frac{1}{d}}\right)=O\left(r^{\frac{1}{d}}\right)
$$

Theorem 7.9 gives another proof of the Szemerédi-Trotter theorem to bound the number of incidences between a set $P$ of $n$ points and a set $L$ of $n$ lines in the plane, this time by partitioning the points instead of the lines: Apply Theorem 7.9 on $P$ with $r=n^{\frac{2}{3}}$ to get a polynomial $f$, with $\operatorname{deg}(f)=O\left(n^{\frac{1}{3}}\right)$. Note that each line of $L$ intersects $O(\sqrt{r})=O\left(n^{\frac{1}{3}}\right)$ components of $\mathbb{R}^{2} \backslash Z(f)$ and each of the $O(r)=O\left(n^{\frac{2}{3}}\right)$ components of $\mathbb{R}^{2} \backslash Z(f)$ contains at most $O\left(\frac{n}{r}\right)=O\left(n^{\frac{1}{3}}\right)$ points of $P$. A simple calculation by summing up the incidences across each component induced by $f$ shows that the overall number of incidences is bounded by $O\left(n^{\frac{4}{3}}\right)$.

On the computational side, an efficient algorithm for computing the partition guaranteed by Theorem 7.9 was discovered by Agarwal et al. [6], who then used it to construct efficient data structures for answering range queries with constantcomplexity semi-algebraic sets as ranges, in time close to $O\left(n^{1-\frac{1}{d}}\right)$.

We now move on to our last topic in this section, a recent beautiful theorem of Guth [196] which gives a very general theorem that implies many statements that were previously regarded as unconnected.

Theorem 7.10. Let $\Gamma$ be a set of $k$-dimensional varieties in $\mathbb{R}^{d}$, each defined by at most $m$ polynomial equations of degree at most $D$. For any parameter $r \geq 1$, there exists a d-variate polynomial $f$ of degree $O\left(r^{\frac{1}{d-k}}\right)$ so that each component of $\mathbb{R}^{d} \backslash Z(f)$ intersects at most $\frac{|\Gamma|}{r}$ varieties of $\Gamma$. The constant in the asymptotic notation depends on $D, m$, and $d$.

Note that this theorem implies Theorem 7.9 by setting $k=0$, and it implies Theorem 7.5 (strictly speaking, a polynomial version of it where $\mathbb{R}^{d}$ is partitioned into the components of $\mathbb{R}^{d} \backslash Z(f)$ instead of simplices) by setting $k=d-1$. See 63 . for an exciting new alternative proof of Theorem 7.10 that extends it to a setting with several algebraic varieties.

We present a brief sketch of the proof of Theorem 7.10, which builds upon the proof of Theorem 7.9 in a natural way. The goal, as before, is to find polynomials $f_{0}, \ldots, f_{s}$, where $s \leq \log r^{\frac{d}{d-k}}$ and $\operatorname{deg}\left(f_{i}\right) \leq 2^{\frac{i}{d}}$ for each $i=0, \ldots, s$. Then the required polynomial will be

$$
f=\prod_{i=0}^{\log r^{\frac{d}{d-k}}} f_{i} \quad \text { with } \quad \operatorname{deg}(f) \leq \sum_{i=0}^{\log r^{\frac{d}{d-k}}} \operatorname{deg}\left(f_{i}\right) \leq \sum_{i=0}^{\log r^{\frac{d}{d-k}}} O\left(2^{\frac{i}{d}}\right)=O\left(r^{\frac{1}{d-k}}\right),
$$

as required. To see the idea behind the proof of Theorem 7.10, trace the proof of Theorem 7.9 backward: the polynomials $f_{i}$ 's were constructed using Theorem 7.4 , whose proof used the ham sandwich theorem in a suitably high dimension, whose proof identified the coefficients of the polynomial with points of the sphere $\mathbb{S}^{d^{\prime}}$ in a suitable dimension $d^{\prime}$ and then applied the Borsuk-Ulam theorem. Note that the $f_{i}$ 's were constructed independently via an iterative argument, where the first $f_{0}$ was used to partition the given point set $P$ into two sets. These two sets were then equipartitioned with $f_{1}$ and so on. This approach relied crucially on the fact that a point, outside the zero set of any polynomial $f_{i}$, lies in precisely one cell induced by their product. This fact fails for $k$-dimensional varieties when $k>0$ : Assume one has constructed polynomials $f_{0}, \ldots, f_{s^{\prime}-1}, s^{\prime}<s$, such that each 
component induced by $f^{\prime}=\prod_{i=0}^{s^{\prime}-1} f_{i}$ has the same number of incidences with the varieties in $\Gamma$. Then, even if the next polynomial $f_{s^{\prime}}$ equipartitions the incidences of each component of $f^{\prime}$, that does not imply that each component induced by the polynomial $f^{\prime} \cdot f_{s^{\prime}}$ will have the same number of incidences with $\Gamma$.

The key idea here is to compute $f_{0}, \ldots, f_{s}$ simultaneously. Thus we will identify, as before, the coefficients of each $f_{i}$ with some $\mathbb{S}^{d_{i}}$, but now instead of applying the Borsuk-Ulam theorem separately for each $i$, we will consider the product of these spheres and apply the following natural variant of the Borsuk-Ulam theorem to get the required polynomials $f_{0}, \ldots, f_{s}$ in one step. For an integer $s \geq 1$, let $X_{s}=\prod_{i=1}^{s} \mathbb{S}^{2^{i-1}}$, and for each $i \in[s]$, define the functions

$$
F l_{i}\left(\boldsymbol{x}_{1}, \ldots, \boldsymbol{x}_{i-1}, \boldsymbol{x}_{i}, \boldsymbol{x}_{i+1}, \ldots, \boldsymbol{x}_{s}\right)=\left(\boldsymbol{x}_{1}, \ldots, \boldsymbol{x}_{i-1},-\boldsymbol{x}_{i}, \boldsymbol{x}_{i+1}, \ldots, \boldsymbol{x}_{s}\right) .
$$

For each $v \in \mathbb{Z} \backslash\{0\}$, let $f_{v}: X_{s} \rightarrow \mathbb{R}$ be a continuous function with the property that $f_{v}\left(F l_{i}(\boldsymbol{x})\right)=(-1)^{v_{i}} f_{v}(\boldsymbol{x})$. Then there exists a point $\boldsymbol{x} \in X_{s}$ where $f_{v}(\boldsymbol{x})=0$ for all $v \in \mathbb{Z} \backslash\{0\}$.

We conclude this section with an open problem - the affine version of Theorem 7.10 .

Conjecture 7.11. For any set $\mathcal{H}_{1}$ of $n k_{1}$-dimensional flats, any set $\mathcal{H}_{2}$ of $m$ $k_{2}$-dimensional flats in $\mathbb{R}^{d}$, and any integer $r$, there exists a partition of $\mathbb{R}^{d}$ into $O\left(r^{d}\right)$ simplices such that (a) each simplex intersects $O\left(\frac{n}{r^{d-k_{1}}}\right)$ flats of $\mathcal{H}_{1}$, and (b) each flat in $\mathcal{H}_{2}$ intersects $O\left(r^{k_{2}}\right)$ simplices.

7.4. Depth of point sets. Data science aims to understand the features of data sets. The goal of data depth measures is to generalize the idea of the statistical median of a set of reals to higher dimensions: the data consists of a finite set $P$ of points in $\mathbb{R}^{d}$, and the goal is to compute a point $\boldsymbol{q} \in \mathbb{R}^{d}$ that is a combinatorial center of the data $P$. As we will see, there are several natural ways to measure data depth, and they are related to each other in sometimes surprising ways.

Figure 14 shows a set of points in $\mathbb{R}^{2}$ (circles), with combinatorial centers under three different measures: halfspace depth (cross), simplicial depth (box), and Oja depth (shaded disk). As the figure shows, the points for these three measures are geometrically close. Given integers $d$ and $n$, let $\mathcal{P}_{n}^{d}$ be the point set in $\mathbb{R}^{d}$ of size $n$,
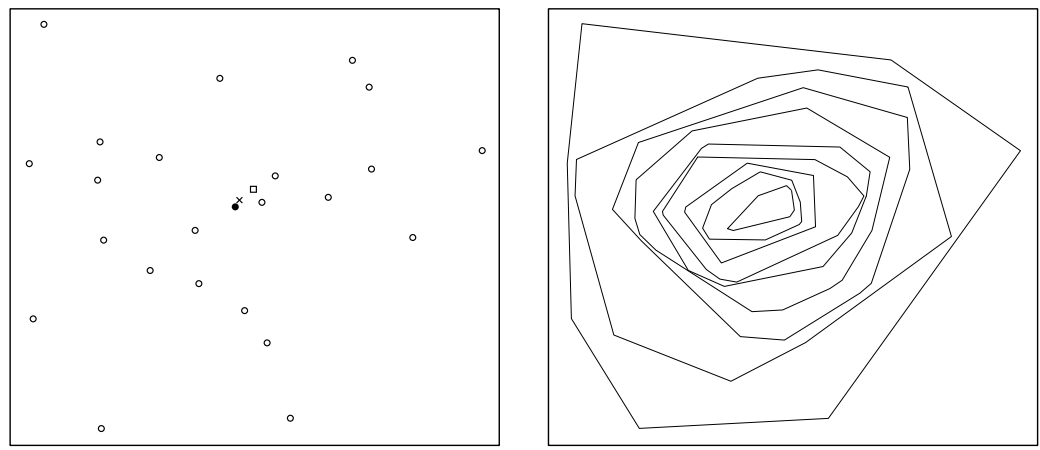

FiguRE 14. Left: A set of points in $\mathbb{R}^{2}$ together with three centers under halfspace (cross), simplicial (box) and Oja (disk) depth measures. Right: The $\beta$-deep regions under halfspace depth measure. 
with at least $\left\lfloor\frac{n}{d+1}\right\rfloor$ points placed at each of the vertices of the standard simplex. Slightly perturb each point so that all $n$ points are distinct, and in general position. This point set will be very useful for the remainder of this section, as it essentially captures both the intuition as well as the worst-case behavior with respect to many depth measures.

Halfspace depth. Given a set $P$ of $n$ points in $\mathbb{R}^{d}$, the halfspace depth of a point $\boldsymbol{q} \in \mathbb{R}^{d}$ with respect to $P$ is the minimum number of points of $P$ in any closed halfspace containing $q$ :

$$
\text { HALFspaCe-Depth }(\boldsymbol{q}, P)=\min _{\text {halfspace } H, \boldsymbol{q} \in H}|H \cap P| \text {. }
$$

Define the halfspace depth of $P$ as the maximum halfspace depth of any point in $\mathbb{R}^{d}$ (this has also been called Tukey depth [375]). The separation theorem implies that any point outside $\operatorname{conv}(P)$ has halfspace depth 0 . It is a nontrivial fact, first shown by Rado in 1947, that points of high halfspace depth exist for every point set.

Theorem 7.12 (Centerpoint theorem [320]). Any set of $n$ points in $\mathbb{R}^{d}$ has halfspace depth at least $\left\lceil\frac{n}{d+1}\right\rceil$.

Recall such a point is called a centerpoint of $P$, and we saw its importance and generalizations in various places within this survey (e.g., in Section 6.5.5). It turns out the centerpoint theorem is optimal, in the sense that the bound $\left\lceil\frac{n}{d+1}\right\rceil$ cannot be improved; $\mathcal{P}_{n}^{d}$ is an example of a point set where it is not possible to do better. By now there are several proofs of the centerpoint theorem: using Brouwer's fixed-point theorem [91, using Helly's theorem [257, following from Tverberg's theorem, and an elementary extremal argument by induction on the dimension $d$ [294]. Perhaps the following proof is the simplest: observe that any point $q \in \mathbb{R}^{d}$ hitting all convex objects containing greater than $\frac{d}{d+1} n$ points of $P$ is a centerpoint, whose existence now follows from Helly's theorem.

The centerpoint theorem and its generalizations have found several applications in combinatorial geometry, statistics, geometric algorithms, meshing, and related areas. A beautiful example is by Miller and Thurston [279, who showed that given a set $\mathcal{D}$ of $n$ disjoint disks in the plane, there exists another disk $B \subset \mathbb{R}^{2}$ intersecting $O(\sqrt{n})$ disks of $\mathcal{D}$, and with at least $\frac{n}{4}$ disks of $\mathcal{D}$ lying completely in the two connected components of $\mathbb{R}^{2}$ induced by $B$. To see this, use an inverse stereographic projection to lift the centers of the disks of $\mathcal{D}$ to a set $P$ of points lying on a carefully chosen sphere in $\mathbb{R}^{3}$. Then, with high probability, the image of the intersection of a random hyperplane through the centerpoint of $P$ with the sphere is the required disk $D$ !

A point of highest halfspace depth with respect to $P$ is called a Tukey median of $P$. It may not be unique. In general, the set of points of halfspace depth at least $\beta n$, for $0 \leq \beta \leq 1$, forms a convex region called the $\beta$-deep region of $P$. It is the intersection of all halfspaces containing more than $(1-\beta) n$ points of $P$. Each facet of this region is supported by a hyperplane that passes through $d$ points of $P$; Figure 14 shows that set of all such regions for the earlier point set. MartínezSandoval and Tamam, gave a generalization of Tukey depth in [254] which connects depth to fractional Helly theorems.

Algorithms. There has been considerable work on the algorithmic question of computing points of large halfspace depth. We first discuss the case in $\mathbb{R}^{2}$, which is 
by now settled. A centerpoint of $n$ points in $\mathbb{R}^{2}$ can be computed in $O(n)$ time [215], the key tool being the linear-time algorithm for computing ham sandwich partitions of two point sets in the plane. Chan [93] gave an $O(n \log n)$ time randomized algorithm for computing a point of the highest halfspace depth, i.e., a Tukey median for a set of points in the plane. The set of all depth contours of $n$ points in $\mathbb{R}^{2}$ can be computed in time $O\left(n^{2}\right)$ 280. A real-time GPU-based algorithm for computing the set of all deep regions of a two-dimensional point set was given in [237. Turning to $\mathbb{R}^{d}, d \geq 3$, the current best algorithms for both computing any centerpoint and the highest depth point take $O\left(n^{d-1}\right)$ time [93. Clarkson et al. [112] presented an iterative method to compute approximate centerpoints: the algorithm constructs a $(d+2)$-ary tree $T$, where the $n$ leaves of $T$ are the input points, and each internal node represents the Radon point (namely, the unique point lying in the common intersection of the convex hulls of the two Tverberg partitions of these $d+2$ points) of its $d+2$ children. This method was improved to the current best algorithm [287] which computes a point of halfspace depth at least $\frac{n}{4(d+1)^{3}}$ in time $d^{O(\log d)} n$. In fact, this method computes an approximate Tverberg partition; namely, a partition of $P$ into $\left\lceil\frac{n}{4(d+1)^{3}}\right\rceil$ sets whose convex hulls have a common intersection.

Open Problem 7.13. Can a centerpoint of $n$ points in $\mathbb{R}^{3}$ be computed in $\tilde{O}(n)$ time?

Simplicial Depth. A straightforward implication of Proposition 3.1 is that given a set $P$ of $n$ points in $\mathbb{R}^{d}$, any point $\boldsymbol{q} \in \operatorname{conv}(P)$ lies in the convex hull of at least $n-d$ tuples of $\left(\begin{array}{c}P \\ d+1\end{array}\right)$. In fact, any centerpoint must be contained in many more simplices spanned by $(d+1)$-tuples of points in $P$. Not surprisingly, the number of $(d+1)$-tuples of $P$ whose convex hull contains $\boldsymbol{q}$ is positively correlated to the halfspace depth of $\boldsymbol{q}$. This leads to the related depth measure of a simplicial depth, of first defined by Liu [244], which is the number of simplices spanned by points of $P$ containing a given point,

$$
\operatorname{Simplicial-Depth}(\boldsymbol{q}, P)=\left|\left\{Q \in\left(\begin{array}{c}
P \\
d+1
\end{array}\right): \boldsymbol{q} \in \operatorname{conv}(Q)\right\}\right| .
$$

The simplicial depth of $P$ is the highest simplicial depth of any point $\boldsymbol{q} \in \mathbb{R}^{d}$. As mentioned earlier, there is a close relation between halfspace depth and simplicial depth; the current best bound [389] shows that a point of halfspace depth $\tau n$ has simplicial depth at least

$$
\frac{(d+1) \tau^{d}-2 d \tau^{d+1}}{(d+1) !} \cdot n^{d+1}-O\left(n^{d}\right)
$$

Bárány [40] showed that the colorful Carathéodory's theorem together with Tverberg's theorem implies that there always exists a point contained in at least $\frac{1}{d !(d+1)^{d+1}} \cdot n^{d+1}$ simplices spanned by $P$. Let $c_{d}$ be a constant such that any set $P$ of $n$ points has simplicial depth at least $c_{d} \cdot n^{d+1}$. The optimal dependency on $c_{d}$ is a long-standing open problem. Bukh, Matoušek, and Nivash [86] constructed $n$ points in $\mathbb{R}^{d}$ so that no point in $\mathbb{R}^{d}$ is contained in, up to lower-order terms, more than $\left(\frac{n}{d+1}\right)^{d+1}$ simplices defined by $P$. Furthermore, they conjectured that this is the optimal bound. 
Conjecture 7.14. Any set of $n$ points in $\mathbb{R}^{d}$ has simplicial depth at least

$$
\left(\left\lceil\frac{n}{(d+1)}\right\rceil\right)^{d+1} \text {. }
$$

For $d=2$, a positive answer to the above conjecture was known already in 1984 by Boros and Füredi 73 . Bukh [84] gave a beautiful short proof: the required point set is the common intersection point of three lines in $\mathbb{R}^{2}$, having a common intersection and where each of the six induced cones contain at least $\frac{n}{6}$ points of $P$; the existence of such three lines follows via an elementary topological argument. For $d=3$, an elementary argument shows that $c_{3} \geq 0.0023$ [53]. Using algebraic topology machinery, Gromov 193 improved the bound to the value $c_{3} \geq 0.0026$. This bound for $\mathbb{R}^{3}$ has since been improved even further by Matoušek and Wagner 266 to 0.00263 , and then by Král et al. 235] to 0.0031. In fact, Gromov proves the bound for general $d$, showing that

$$
c_{d} \geq \frac{2 d}{(d+1) !^{2}(d+1)} .
$$

His proof has since been simplified by Karasev [227. Using the concepts of Gale diagrams and secondary polytopes, one can observe that the concept of simplicial depth is equivalent to a different maximization problem: Given a set of points on the sphere $\mathbb{S}^{d}$, what is the triangulation that uses those points (but possibly not all) as vertices that have the largest number of $d$-dimensional simplices? (See 132, Chapter 5] and the references therein.)

We conclude this discussion of simplicial depth with colorful simplicial depth, which was introduced by Deza et al. 139 . Consider a set of points $P$ in $\mathbb{R}^{d}$ partitioned into $(d+1)$ color classes $P=P_{0} \cup \cdots \cup P_{d}$. Suppose that $P$ has the property that the origin $\boldsymbol{o}$ is in the relative interior of each $\operatorname{conv}\left(P_{i}\right)$, for $0 \leq i \leq d$. Recall from Section 3.1 that a colorful simplex is a simplex where each of the vertices comes from a different $P_{i}$. While the colorful Carathéodory theorem asserts the existence of at least one colorful simplex containing $\boldsymbol{o}$, one can further ask about the number of distinct colorful simplices containing $\boldsymbol{o}$ that must always exist. Define the colorful simplicial depth of $P$, denoted ColorfulSimp-Depth $(P)$, as the number of colorful simplices in $P$ containing $\boldsymbol{o}$. Deza et al. [139] proposed some lower bounds on the colorful simplicial depth, and they conjectured that if $\left|P_{i}\right|=d+1$ for $0 \leq i \leq d$, then $\operatorname{Colorful} \operatorname{Simp-Depth}(P) \geq d^{2}+1$. This was proven by Sarrabezolles [336]. The bound is optimal by the work of Deza et al. [139]. They also conjectured the following upper bound which was shown by Adiprasito et al. [4]: let $P=P_{0} \cup \cdots \cup P_{d}$ be a point set in $\mathbb{R}^{d}$ with $\left|P_{i}\right| \geq 2$ for all $0 \leq i \leq d$. If no colorful simplex $S$ spanned by $P$ of dimension $d-1$ contains the origin $\boldsymbol{o}$ in its convex hull, then $\operatorname{Colorful} \operatorname{Simp-Depth}(P) \leq 1+\prod_{i=0}^{d}\left(\left|P_{i}\right|-1\right)$.

Algorithms. For the case of the plane, computing the simplicial depth of a query point can be done in time $O(n \log n)$ [185, which is optimal. Aloupis et al. 25] presented an algorithm to compute a point of highest simplicial depth in $\mathbb{R}^{2}$ in time $O\left(n^{4}\right)$. Using the fact that finding the highest simplicial depth is Gale dual to the problem of finding a maximum triangulation of points in the sphere, then one can set up an integer program to find a point of largest simplicial depth for any point configuration (see [132, Chapter 8]). A GPU-based algorithm for computing simplicial depth and colorful simplicial depth of point sets in the plane was given in 238 . 
Ray-shooting depth. It turns out that the previous two measures-halfspace depth and simplicial depth - are further related to each other via an even more general depth measure, called ray-shooting depth. Given a set $P$ of $n$ points in $\mathbb{R}^{d}$, let $E_{P}$ be the set of all $\left(\begin{array}{l}n \\ d\end{array}\right)(d-1)$-simplices spanned by points of $P$. Given a point $\boldsymbol{q} \in \mathbb{R}^{d}$ and a direction $\boldsymbol{u} \in \mathbb{S}^{d-1}$, let $r(\boldsymbol{q}, \boldsymbol{u})$ be the half-infinite ray from $\boldsymbol{q}$ in direction $\boldsymbol{u}$. Then the ray-shooting depth of a point $\boldsymbol{q} \in \mathbb{R}^{d}$ is defined as

$$
\operatorname{RAyshooting-Depth}(\boldsymbol{q}, P)=\min _{\boldsymbol{u} \in \mathbb{S}^{d-1}}\left|\left\{e \in E_{P}: r(\boldsymbol{q}, \boldsymbol{u}) \cap e \neq \varnothing\right\}\right| \text {. }
$$

The ray-shooting depth of $P$ is the maximum ray-shooting depth of any point in $\mathbb{R}^{d}$. The notion of ray-shooting depth was first introduced in Fox et al. [166, who proved the following using Brouwer's fixed point theorem.

Theorem 7.15. Any set of $n$ points in $\mathbb{R}^{2}$ has ray-shooting depth at least $\frac{n^{2}}{9}$.

Note that any point realizing the maximum ray-shooting depth must have halfspace depth at least $\frac{n}{3}$ and simplicial depth at least $\frac{n^{3}}{27}$ : Let $\boldsymbol{q}$ be a point with ray-shooting depth at least $\frac{n^{2}}{9}$. Then any line through $\boldsymbol{q}$ must intersect at least $\frac{2 n^{2}}{9}$ segments in $E_{P}$, so both halfspaces defined by it must contain at least $\frac{n}{3}$ points. For simplicial depth, consider, for each point $\boldsymbol{p} \in P$, the ray from $\boldsymbol{q}$ in the direction $\overrightarrow{\boldsymbol{p}} \mathbf{q}$. Then for every edge $\left\{\boldsymbol{p}_{i}, \boldsymbol{p}_{j}\right\}$ that intersects this ray, the triangle defined by $\left\{\boldsymbol{p}, \boldsymbol{p}_{i}, \boldsymbol{p}_{j}\right\}$ must contain $\boldsymbol{q}$. Summing up these triangles over all points, each triangle can be counted three times, and so $\boldsymbol{q}$ lies in at least $\frac{n^{3}}{27}$ distinct triangles spanned by $P$.

The problem of showing the existence of a point with large ray-shooting depth is open in higher dimensions.

Conjecture 7.16. Any set of $n$ points in $\mathbb{R}^{d}$ has ray-shooting depth at least $\left(\left\lceil\frac{n}{d+1}\right\rceil\right)^{d}$.

Other notions of ray-shooting depth for convex sets, instead of points sets, were studied in [174].

Algorithms. The proof in [166] is topological and does not give a method for computing such a point. A combinatorial proof, together with efficient algorithms were obtained in Mustafa et al. 296, where they showed how compute a point of ray-shooting depth at least $\frac{n^{2}}{9}$ in time $\tilde{O}\left(n^{2}\right)$.

Oja depth. It turns out that ray-shooting depth is related to another older depth measure, the Oja depth of a point set, first defined by Oja 307. Assume, without loss of generality, that $\operatorname{vol}(\operatorname{conv}(P))=1$. Then define the Oja depth of a point $\boldsymbol{q}$ with respect to $P$ as

$$
\text { OJA-Depth }(\boldsymbol{q}, P)=\sum_{\substack{P^{\prime} \subseteq P \\\left|P^{\prime}\right|=d}} \operatorname{vol}\left(\operatorname{conv}\left(P^{\prime} \cup\{\boldsymbol{q}\}\right)\right) .
$$

The Oja depth of $P$ is the minimum Oja depth over all $\boldsymbol{q} \in \mathbb{R}^{d}$. It is easy to see that the Oja depth of $\mathcal{P}_{n}^{d}$ is at least $\left(\frac{n}{d+1}\right)^{d}$. The conjecture 99] is that the lower bound given by $\mathcal{P}_{n}^{d}$ is essentially tight.

Conjecture 7.17. The Oja depth of any set of $n$ points in $\mathbb{R}^{d}$ is at most $\left(\frac{n}{d+1}\right)^{d}$. 
The conjecture has been resolved only for the case $d=2$ by Mustafa et al. 298]. For general $d$, it can be shown that the center of mass of $P$ has Oja depth at most $\left(\begin{array}{l}n \\ d\end{array}\right) /(d+1)$ [99. This estimate can be improved via ray-shooting depth, as the Oja depth of any point $\boldsymbol{q}$ which has ray-shooting depth at least $\frac{n^{2}}{9}$ is at least $\frac{n^{2}}{7.2}$. The reason is the number of triangles spanned by pairs of points in $P$ and the point $\boldsymbol{q}$, containing any point $\boldsymbol{p} \in \mathbb{R}^{2}$, is at most the number of edges spanned by $P$ intersecting the ray $\overrightarrow{\boldsymbol{q}}$, which is at most $\frac{n^{2}}{4}-\frac{n^{2}}{9}=\frac{n^{2}}{7.2}$. Integrating over all $\boldsymbol{p} \in \mathbb{R}^{2}$ gives the required bound. A calculation in $\mathbb{R}^{d}$ gives the current best bound [298]:

Theorem 7.18. Every set of $n$ points in $\mathbb{R}^{d}, d \geq 3$, has Oja depth at most

$$
\frac{2 n^{d}}{2^{d} d !}-\frac{2 d}{(d+1)^{2}(d+1) !}\left(\begin{array}{l}
n \\
d
\end{array}\right)+O\left(n^{d-1}\right) .
$$

Algorithms. For the case $d=2$, Rousseeuw and Ruts [330] presented an $O\left(n^{5} \log n\right)$ time algorithm for computing the lowest depth point, which was then improved to the current best algorithm with running time $O\left(n \log ^{3} n\right)$ [25]. A point of Oja depth at most $\frac{n^{2}}{9}$ can be computed in $O(n \log n)$ time [298. For general $d$, various heuristics for computing points with low Oja depth were given by Ronkainen, Oja, and Orponen 327.

Regression depth. The next depth measure, unlike earlier measures, is a combinatorial analogue of fitting a hyperplane through a set of points. Therefore it will be more convenient to state it in the dual. Given a point $\boldsymbol{p} \in \mathbb{R}^{d}$, let $p^{*}$ be its dual hyperplane, and for a set of points $P$, let $P^{*}=\left\{p^{*}: \boldsymbol{p} \in P\right\}$. Then define the regression depth of a point as

$$
\operatorname{RegRession-Depth}(\boldsymbol{q}, P)=\min _{\boldsymbol{u} \in \mathbb{S}^{d-1}}\left|\left\{H \in P^{*}: r(\boldsymbol{q}, \boldsymbol{u}) \cap H \neq \varnothing\right\}\right| .
$$

The regression depth of a set $P$ of points in $\mathbb{R}^{d}$ is the maximum regression depth of any point $\boldsymbol{q} \in \mathbb{R}^{d}$. It was introduced by Rousseeuw and Hubert [331, who showed that any set $P$ of $n$ points in $\mathbb{R}^{2}$ has regression depth at least $\left\lceil\frac{n}{3}\right\rceil$. Their proof is elegant: given the set $P$ of $n$ points, let $P_{1}, P_{2}, P_{3}$ be a partition of $P$ by consecutive $x$-coordinate values, and where $\left|P_{i}\right| \leq\left\lceil\frac{n}{3}\right\rceil$ for $i=1,2,3$. Then the required line is the ham sandwich cut of the two sets $P_{1} \cup P_{2}$ and $P_{2} \cup P_{3}$. The optimal bound for general $d$ was discovered later.

Theorem 7.19 ([27, 224, 283]). Any set of $n$ points in $\mathbb{R}^{d}$ has regression depth at least $\left\lceil\frac{n}{d+1}\right\rceil$.

Given a set $X \subseteq \mathbb{R}^{d}$ and a point $q \in \mathbb{R}^{d}$, the closest point in $X$ to $q$ (if it exists) is denoted by $\mathrm{c}(q, X)$. The proof in 224 deduces it from the centerpoint theorem: define the function $f(\boldsymbol{q})$ that maps $\boldsymbol{q} \in \mathbb{R}^{d}$ to a centerpoint of the set $\left\{\mathrm{c}\left(\boldsymbol{q}, p^{*}\right): \boldsymbol{p} \in P\right\}$. This can be done so that $f(\cdot)$ is continuous and maps a sufficiently large ball to itself. Then observe that the dual of any fixed point of $f(\cdot)$ is the required hyperplane.

Algorithms. The method of 331 gives a linear-time algorithm for computing a point of regression depth at least $\left\lceil\frac{n}{3}\right\rceil$ immediately, as it uses only ham sandwich cuts. A point of maximum regression depth in $\mathbb{R}^{2}$ can be computed in time $O(n \log n)$ 382, improving upon an earlier $O\left(n \log ^{2} n\right)$ time algorithm [240. For $d \geq 3$, the best algorithm takes time $O\left(n^{d}\right)[382$ to compute a point of maximum regression depth. 
The $k$-centerpoint conjectures. It turns out that many of the depth measures discussed are special cases of the following more general conjecture, first proposed by Mustafa et al. [297]; see also the related paper [226].

Conjecture 7.20. For any set $P$ of $n$ points in $\mathbb{R}^{d}$ and any integer $0 \leq k \leq d$, there exists a point $\boldsymbol{q} \in \mathbb{R}^{d}$ such that any $(d-k)$-half flat through $\boldsymbol{q}$ intersects at least $\left(\left\lceil\frac{n}{d+1}\right\rceil\right)^{k+1}$ of the $k$-simplices spanned by $P$.

The case $k=0$ corresponds to halfspace depth, $k=d$ corresponds to simplicial depth, and $k=d-1$ corresponds to ray-shooting depth.

It is not hard to show that given a set $P$ of $n$ points in $\mathbb{R}^{d}$, and an integer $0 \leq k \leq d-1$, there exists a point $\boldsymbol{q} \in \mathbb{R}^{d}$ such that any $(d-k)$-half flat through $\boldsymbol{q}$ intersects at least

$$
\max \left\{\left(\begin{array}{c}
\frac{n}{d+1} \\
k+1
\end{array}\right), \quad \frac{2 d}{(d+1)(d+1) !\left(\begin{array}{c}
n \\
d-k
\end{array}\right)} \cdot\left(\begin{array}{c}
n \\
d+1
\end{array}\right)\right\}
$$

$k$-simplices spanned by $P$. For simplicity, assume that $|P|$ is a multiple of $(d+1)$. The proof follows from the use of Tverberg's theorem to partition $P$ into $t=\frac{n}{(d+1)}$ sets $P_{1}, \ldots, P_{t}$ such that there exists a point $\boldsymbol{q}$ with $\boldsymbol{q} \in \operatorname{conv}\left(P_{i}\right)$ for all $i$. Consider any $(d-k)$-dimensional half-flat $\mathcal{F}$ through $\boldsymbol{q}$, where $\partial \mathcal{F}$ is a $(d-k-1)$-dimensional flat containing $\boldsymbol{q}$. Project $\mathcal{F}$ onto a $(k+1)$-dimensional subspace $\mathcal{H}$ orthogonal to $\partial \mathcal{F}$ such that the projection of $\mathcal{F}$ is a ray $\boldsymbol{r}$ in $\mathcal{H}$, and $\partial \mathcal{F}$ and $\boldsymbol{q}$ are projected to the point $\boldsymbol{q}^{\prime}$. And let $P_{1}^{\prime}, \ldots, P_{t}^{\prime}$ be the projected sets whose convex hulls now contain the point $\boldsymbol{q}^{\prime}$. Then note that the $k$-dimensional simplex spanned by $(k+1)$ points $Q^{\prime} \subset P^{\prime}$ intersects the ray $\boldsymbol{r}$ if and only if the $k$-dimensional simplex defined by the corresponding set $Q$ in $\mathbb{R}^{d}$ intersects the flat $\mathcal{F}$. Now apply the single-point version (i.e., given any point $s \in \mathbb{R}^{d}$ and $d$ sets $P_{1}, \ldots, P_{d}$ in $\mathbb{R}^{d}$ such that each $\operatorname{conv}\left(P_{i}\right)$ contains the origin, there exists a $d$-simplex spanned by $s$ and one point from each $P_{i}$ which also contains the origin) of a colorful Carathéodory theorem to every $(k+1)$-tuple of sets, say $P_{1}^{\prime}, \ldots, P_{k+1}^{\prime}$, together with the point $s$ at infinity in the direction antipodal to the direction of $\boldsymbol{r}$ to get a "colorful" simplex, defined by $\boldsymbol{s}$ and one point from each $P_{i}^{\prime}$, and containing $\boldsymbol{q}^{\prime}$. Then the ray $\boldsymbol{r}$ must intersect the $k$-simplex defined by the $(k+1)$ points of $P^{\prime}$, and so the corresponding points of $P$ in $\mathbb{R}^{d}$ span a $(k+1)$-simplex intersecting $\mathcal{F}$. In total, we get $\left(\begin{array}{c}n /(d+1) \\ k+1\end{array}\right)$ of the $k$-simplices intersecting $\mathcal{F}$. Another way is to use the simplicial depth bound of Gromov, that given any set $P$ of $n$ points in $\mathbb{R}^{d}$, there exists a point $\boldsymbol{q}$ lying in $2 d /((d+1)(d+1) !) \cdot\left(\begin{array}{c}n \\ d+1\end{array}\right) d$-simplices. Now take any $(d-k)$-half flat through $\boldsymbol{q}$. It must intersect at least one $k$-simplex of each $d$-simplex containing it, where each $k$-simplex is counted at most $\left(\begin{array}{c}n \\ d-k\end{array}\right)$ times. This implies the stated bound.

In the plane $(d=2)$ the centerpoint theorem can be restated as follows: Given a set $P$ of $n$ points in the plane, there exists a point $q$ such that if you take any line $L$ passing through $q$, and move it continuously until it arrives outside the convex hull of $P$, then along this motion, the line will intersect at least $n / 3$ points of $P$. The following more general statement has also been conjectured in [297]:

Conjecture 7.21. Given a set $P$ of $n$ points in $\mathbb{R}^{d}$ and an integer $0 \leq k \leq d$, there exists a point $q \in \mathbb{R}^{d}$ such that the following holds: Let $F_{q}, F_{o}$ be two $(d-k-1)$ flats, such that $q \in F_{q}$ and $F_{o}$ does not intersect the convex hull of $P$. Then any 
continuous motion family of $(d-k-1)$-flats, starting at $F_{q}$ and ending at $F_{o}$, must intersect at least $\left(\left\lceil\frac{n}{d+1}\right\rceil\right)^{k+1} k$-simplices spanned by $P$.

In Conjecture 7.21, the case $k=d$ gives a " -1 "-flat moving to infinity, which can be treated as a stationary point. The validity of these conjectures for the planar case $d=2$ follows from the work of Gromov [193.

\section{ACKNOWLEDGMEnTS}

The authors are truly grateful to the two anonymous referees who provided a very large and very detailed set of comments, insights, and corrections. Their reading of this work was invaluable and it took a lot of effort and time. Very special thanks to Peter Landweber who gave us numerous corrections too. We are also grateful to the following colleagues who gave us support and many comments and references: Ian Agol, Imre Bárány, Pavle Blagojević, Boris Bukh, Sabrina Enriquez, Florian Frick, Alexei Garber, Tommy Hogan, Andreas Holmsen, Wolfgang Mulzer, Oleg Musin, Deborah Oliveros, János Pach, Dőmőtőr Pálvőlgyi, Axel Parmentier, Lily Silverstein, Pablo Soberón, Francis Su, Uli Wagner, and Günter M. Ziegler. We are grateful for their help. The first author thanks Labex BEZOUT and the CERMICS research center at École National des Ponts et Chaussées for the support received and the enjoyable and welcoming environment in which the topics in this paper were discussed and built over several visits.

\section{ABout THE AUTHORS}

Jesús A. De Loera is professor of mathematics and a member of the graduate group of computer science at University of California, Davis. In 1995 he obtained his $\mathrm{PhD}$ in applied mathematics from Cornell University. De Loera's research includes combinatorics, algorithms, convexity, applied algebra, and optimization. He is a fellow of the AMS.

Xavier Goaoc is professor of computer science at Université de Lorraine and a member of Institut Universitaire de France. His research is in discrete and computational geometry.

Frédéric Meunier is professor of operations research at École des Ponts ParisTech. He obtained his $\mathrm{PhD}$ in 2006 from Grenoble University. His research interests are in combinatorics, operations research, and transportation.

Nabil H. Mustafa is professor of computer science at ESIEE (École Supérieure d'Ingénieurs en Électrotechnique et Électronique) Paris. He obtained his PhD in 2004 from the department of computer science at Duke University. His research interests include approximation algorithms, combinatorics, discrete and computational geometry.

\section{REFERENCES}

[1] K. I. Aardal, S. P. M. van Hoesel, A. M. C. A. Koster, C. Mannino, and A. Sassano, Models and solution techniques for frequency assignment problems, Ann. Oper. Res. 153 (2007), 79-129, DOI 10.1007/s10479-007-0178-0. MR2329980 4458

[2] M. Adamaszek and J. A. Barmak, On a lower bound for the connectivity of the independence complex of a graph, Discrete Math. 311 (2011), no. 21, 2566-2569, DOI 10.1016/j.disc.2011.06.010. MR2832154 461

[3] K. Adiprasito, I. Bárány, and N. Mustafa, Theorems of Carathéodory, Helly, and Tverberg without dimension, arXiv:1806.08725 (2018). 1438 
[4] K. A. Adiprasito, P. Brinkmann, A. Padrol, P. Paták, and Z. Patáková, and R. Sanyal, Colorful simplicial depth, Minkowski sums, and generalized Gale transforms, Int. Math. Res. Not. IMRN (2017), $\operatorname{rnx} 184.1489$

[5] I. Adler, The equivalence of linear programs and zero-sum games, Internat. J. Game Theory 42 (2013), no. 1, 165-177, DOI 10.1007/s00182-012-0328-8. MR3028051 467.

[6] P. K. Agarwal, J. Matoušek, and M. Sharir, On range searching with semialgebraic sets. II, SIAM J. Comput. 42 (2013), no. 6, 2039-2062, DOI 10.1137/120890855. MR3123833 1485

[7] R. Aharoni, E. Berger, and R. Ziv, Independent systems of representatives in weighted graphs, Combinatorica 27 (2007), no. 3, 253-267, DOI 10.1007/s00493-007-2086-y. MR2345810 461

[8] R. Aharoni and R. Holzman, Fractional kernels in digraphs, J. Combin. Theory Ser. B 73 (1998), no. 1, 1-6, DOI 10.1006/jctb.1997.1731. MR1620603 1463

[9] M. Ahmed, J. De Loera, and R. Hemmecke, Polyhedral cones of magic cubes and squares, Discrete and computational geometry, Algorithms Combin., vol. 25, Springer, Berlin, 2003, pp. 25-41, DOI 10.1007/978-3-642-55566-4_2. MR2038468 1419

[10] M. Aigner and G. M. Ziegler, Proofs from The Book, 5th ed., Springer-Verlag, Berlin, 2014. Including illustrations by Karl H. Hofmann. MR.3288091 1463

[11] J. Aisenberg, M. L. Bonet, and S. Buss, 2-d Tucker is PPA-complete. Electronic Colloquium on Computational Complexity (ECCC) 22 (2015), 163. 1433

[12] I. Aliev, R. Bassett, J. A. De Loera, and Q. Louveaux, A quantitative Doignon-BellScarf theorem, Combinatorica 37 (2017), no. 3, 313-332, DOI 10.1007/s00493-015-3266-9. MR 3666782 442

[13] I. Aliev, J. A. De Loera, F. Eisenbrand, T. Oertel, and R. Weismantel, The support of integer optimal solutions, SIAM J. Optim. 28 (2018), no. 3, 2152-2157, DOI 10.1137/17M1162792. MR.3835599 465

[14] I. Aliev, J. A. De Loera, T. Oertel, and C. O'Neill, Sparse solutions of linear Diophantine equations, SIAM J. Appl. Algebra Geom. 1 (2017), no. 1, 239-253, DOI 10.1137/16M1083876. MR3633776 4437

[15] M. Alishahi, Colorful subhypergraphs in uniform hypergraphs, Electron. J. Combin. 24 (2017), no. 1, Paper 1.23, 26. MR.3609193 1426 430

[16] M. Alishahi and H. Hajiabolhassan, On the chromatic number of general Kneser hypergraphs, J. Combin. Theory Ser. B 115 (2015), 186-209, DOI 10.1016/j.jctb.2015.05.010. MR3383256 4460

[17] M. Alishahi, H. Hajiabolhassan, and F. Meunier, Strengthening topological colorful results for graphs, European J. Combin. 64 (2017), 27-44, DOI 10.1016/j.ejc.2017.03.011. MR.3658818 1460

[18] M. Alishahi and F. Meunier, Fair splitting of colored paths, Electron. J. Combin. 24 (2017), no. 3, Paper 3.41, 8. MR3691558 157 461

[19] N. Alon, Splitting necklaces, Adv. in Math. 63 (1987), no. 3, 247-253, DOI 10.1016/00018708(87)90055-7. MR877785 1457

[20] N. Alon, I. Bárány, Z. Füredi, and D. J. Kleitman, Point selections and weak $\epsilon$ nets for convex hulls, Combin. Probab. Comput. 1 (1992), no. 3, 189-200, DOI 10.1017/S0963548300000225. MR.1208800 4482

[21] N. Alon, G. Kalai, J. Matoušek, and R. Meshulam, Transversal numbers for hypergraphs arising in geometry, Adv. in Appl. Math. 29 (2002), no. 1, 79-101, DOI 10.1016/S01968858(02)00003-9. MR 1921545 441

[22] N. Alon and D. J. Kleitman, Piercing convex sets and the Hadwiger-Debrunner $(p, q)$ problem, Adv. Math. 96 (1992), no. 1, 103-112, DOI 10.1016/0001-8708(92)90052-M. MR.1185788 467

[23] N. Alon, D. Moshkovitz, and S. Safra, Algorithmic construction of sets for $k$-restrictions, ACM Trans. Algorithms 2 (2006), no. 2, 153-177, DOI 10.1145/1150334.1150336. MR.2253804 1457

[24] N. Alon and D. B. West, The Borsuk-Ulam theorem and bisection of necklaces, Proc. Amer. Math. Soc. 98 (1986), no. 4, 623-628, DOI 10.2307/2045739. MR.861764 1457

[25] G. Aloupis, S. Langerman, M. Soss, and G. Toussaint, Algorithms for bivariate medians and a Fermat-Torricelli problem for lines, Comput. Geom. 26 (2003), no. 1, 69-79, DOI 10.1016/S0925-7721(02)00173-6. Thirteenth Canadian Conference on Computational Geometry-CCCG'01 (Waterloo, ON). MR1989273 1489 491 
[26] N. Amenta, Helly-type theorems and generalized linear programming, Discrete Comput. Geom. 12 (1994), no. 3, 241-261, DOI 10.1007/BF02574379. ACM Symposium on Computational Geometry (San Diego, CA, 1993). MR1298910 1472

[27] N. Amenta, M. Bern, D. Eppstein, and S.-H. Teng, Regression depth and center points, Discrete Comput. Geom. 23 (2000), no. 3, 305-323, DOI 10.1007/PL00009502. MR.1744506 491

[28] N. Amenta, J. A. De Loera, and P. Soberón, Helly's theorem: new variations and applications, Algebraic and geometric methods in discrete mathematics, Contemp. Math., vol. 685, Amer. Math. Soc., Providence, RI, 2017, pp. 55-95. MR.3625571 416 434442

[29] J. L. Arocha, I. Bárány, J. Bracho, R. Fabila, and L. Montejano, Very colorful theorems, Discrete Comput. Geom. 42 (2009), no. 2, 142-154, DOI 10.1007/s00454-009-9180-4. MR2519872 1436 445

[30] S. Arora and B. Barak, Computational complexity: A modern approach, Cambridge University Press, Cambridge, 2009. MR2500087 432

[31] M. Asada, R. Chen, F. Frick, F. Huang, M. Polevy, D. Stoner, L. H. Tsang, and Z. Wellner, On Reay's relaxed Tverberg conjecture and generalizations of Conway's thrackle conjecture, Electron. J. Combin. 25 (2018), no. 3, Paper 3.16, 14. MR3853868 1445

[32] M. Asada, F. Frick, V. Pisharody, M. Polevy, D. Stoner, L. H. Tsang, and Z. Wellner, Fair division and generalizations of Sperner- and KKM-type results, SIAM J. Discrete Math. 32 (2018), no. 1, 591-610, DOI 10.1137/17M1116210. MR3769696 1428 429 456 458

[33] R. J. Aumann, Subjectivity and correlation in randomized strategies, J. Math. Econom. 1 (1974), no. 1, 67-96, DOI 10.1016/0304-4068(74)90037-8. MR0421694 1450

[34] G. Averkov, On maximal $S$-free sets and the Helly number for the family of S-convex sets, SIAM J. Discrete Math. 27 (2013), no. 3, 1610-1624, DOI 10.1137/110850463. MR.3106473 1476

[35] G. Averkov, B. González Merino, I. Paschke, M. Schymura, and S. Weltge, Tight bounds on discrete quantitative Helly numbers, Adv. in Appl. Math. 89 (2017), 76-101, DOI 10.1016/j.aam.2017.04.003. MR3655733 442 443

[36] G. Averkov and R. Weismantel, Transversal numbers over subsets of linear spaces, Adv. Geom. 12 (2012), no. 1, 19-28, DOI 10.1515/advgeom.2011.028. MR2911157 1440

[37] D. Avis and O. Friedmann, An exponential lower bound for Cunningham's rule, Math. Program. 161 (2017), no. 1-2, Ser. A, 271-305, DOI 10.1007/s10107-016-1008-4. MR.3592779 1473

[38] H. Aziz and S. Mackenzie, A discrete and bounded envy-free cake cutting protocol for any number of agents, 57th Annual IEEE Symposium on Foundations of Computer Science (FOCS) 2016, IEEE Computer Soc., Los Alamitos, CA, 2016, pp. 416-427. MR3631004 1455

[39] E. G. Bajmóczy and I. Bárány, On a common generalization of Borsuk's and Radon's theorem, Acta Math. Acad. Sci. Hungar. 34 (1979), no. 3-4, 347-350 (1980), DOI 10.1007/BF01896131. MR565677 444

[40] I. Bárány, A generalization of Carathéodory's theorem, Discrete Math. 40 (1982), no. 2-3, 141-152, DOI 10.1016/0012-365X(82)90115-7. MR676720 1435 436 488

[41] I. Bárány, Geometric and combinatorial applications of Borsuk's theorem, New trends in discrete and computational geometry. Springer, 1993, pp. 235-249. 459

[42] I. Bárány, P. V. M. Blagojević, and G. M. Ziegler, Tverberg's theorem at 50: extensions and counterexamples, Notices Amer. Math. Soc. 63 (2016), no. 7, 732-739, DOI 10.1090/noti1415. MR3495518 1416 444, 445

[43] I. Bárány, M. Katchalski, and J. Pach, Quantitative Helly-type theorems, Proc. Amer. Math. Soc. 86 (1982), no. 1, 109-114, DOI 10.2307/2044407. MR663877 442

[44] I. Bárány and D. G. Larman, A colored version of Tverberg's theorem, J. London Math. Soc. (2) 45 (1992), no. 2, 314-320, DOI 10.1112/jlms/s2-45.2.314. MR1171558 1446

[45] I. Bárány and J. Matoušek, A fractional Helly theorem for convex lattice sets, Adv. Math. 174 (2003), no. 2, 227-235, DOI 10.1016/S0001-8708(02)00037-3. MR1963693 1440

[46] I. Bárány and S. Onn, Carathéodory's theorem, colourful and applicable, Intuitive geometry (Budapest, 1995), Bolyai Soc. Math. Stud., vol. 6, János Bolyai Math. Soc., Budapest, 1997, pp. 11-21. MR1470753 1435 436 448

[47] I. Bárány and S. Onn, Colourful linear programming and its relatives, Math. Oper. Res. 22 (1997), no. 3, 550-567, DOI 10.1287/moor.22.3.550. MR1467385 1442, 448 
[48] I. Bárány, S. B. Shlosman, and A. Szücs, On a topological generalization of a theorem of Tverberg, J. London Math. Soc. (2) 23 (1981), no. 1, 158-164, DOI 10.1112/jlms/s2-23.1.158. MR602247 444457

[49] I. Bárány and P. Soberón, Tverberg plus minus, Discrete Comput. Geom. 60 (2018), no. 3, 588-598, DOI 10.1007/s00454-017-9960-1. MR3849141 t442

[50] I. Bárány and P. Soberón, Tverberg's theorem is 50 years old: a survey, Bull. Amer. Math. Soc. (N.S.) 55 (2018), no. 4, 459-492, DOI 10.1090/bull/1634. MR.3854075 416 445 446

[51] S. Barman, Approximating Nash equilibria and dense bipartite subgraphs via an approximate version of Carathéodory's theorem, STOC'15-Proceedings of the 2015 ACM Symposium on Theory of Computing, ACM, New York, 2015, pp. 361-369. MR3388215 438 454

[52] A. Barvinok, A course in convexity, Graduate Studies in Mathematics, vol. 54, American Mathematical Society, Providence, RI, 2002. MR 1940576 1420

[53] A. Basit, N. H. Mustafa, S. Ray, and S. Raza, Hitting simplices with points in $\mathbb{R}^{3}$, Discrete Comput. Geom. 44 (2010), no. 3, 637-644, DOI 10.1007/s00454-010-9263-2. MR2679059 489

[54] A. Basu, M. Conforti, G. Cornuéjols, R. Weismantel, and S. Weltge, Optimality certificates for convex minimization and Helly numbers, Oper. Res. Lett. 45 (2017), no. 6, 671-674, DOI 10.1016/j.orl.2017.10.002. MR3724203 1477

[55] A. Basu and T. Oertel, Centerpoints: a link between optimization and convex geometry, Integer programming and combinatorial optimization, Lecture Notes in Comput. Sci., vol. 9682, Springer, Cham, 2016, pp. 14-25, DOI 10.1007/978-3-319-33461-5_2. MR3534718 1477

[56] D. E. Bell, A theorem concerning the integer lattice, Studies in Appl. Math. 56 (1976/77), no. 2, 187-188. MR 0462617 1439

[57] C. Berge and P. Duchet, Seminar, MSHi (Maison des Sciences de l'Homme), 1983. 1463

[58] D. P. Bertsekas, Convex optimization algorithms, Athena Scientific, Belmont, MA, 2015. MR 3558548 1344 446 447

[59] D. Bertsimas and S. Vempala, Solving convex programs by random walks, J. ACM 51 (2004), no. 4, 540-556, DOI 10.1145/1008731.1008733. MR2147847 1476

[60] K. Bezdek and A. Blokhuis, The Radon number of the three-dimensional integer lattice, Discrete Comput. Geom. 30 (2003), no. 2, 181-184, DOI 10.1007/s00454-003-0003-8. MR.2007959 1443

[61] B. J. Birch, On $3 N$ points in a plane, Proc. Cambridge Philos. Soc. 55 (1959), 289-293. MR0109315 442

[62] A. Björner, Topological methods, Handbook of combinatorics, Vol. 2, Elsevier Sci. B. V., Amsterdam, 1995, pp. 1819-1872. MR1373690 422 458459

[63] P. V. M. Blagojević, A. S. D. Blagojević, and G. M. Ziegler, Polynomial partitioning for several sets of varieties, J. Fixed Point Theory Appl. 19 (2017), no. 3, 1653-1660, DOI 10.1007/s11784-016-0322-z. MR3692419 1485

[64] P. V. M. Blagojević, F. Frick, A. Haase, and G. M. Ziegler, Topology of the GrünbaumHadwiger-Ramos hyperplane mass partition problem, Trans. Amer. Math. Soc. 370 (2018), no. 10, 6795-6824, DOI 10.1090/tran/7528. MR3841833 479

[65] P. V. M. Blagojević, F. Frick, A. Haase, and G. M. Ziegler, Hyperplane mass partitions via relative equivariant obstruction theory, Doc. Math. 21 (2016), 735-771. MR3548132 1479

[66] P. V. M. Blagojević, F. Frick, and G. M. Ziegler, Tverberg plus constraints, Bull. Lond. Math. Soc. 46 (2014), no. 5, 953-967, DOI 10.1112/blms/bdu049. MR3262197 441

[67] P. V. M. Blagojević, F. Frick, and G. M. Ziegler, G. M. Barycenters of polytope skeleta and counterexamples to the topological Tverberg conjecture, via constraints, J. Europ. Math. Soc. (to appear) (2017). 435

[68] P. V. M. Blagojević, B. Matschke, and G. M. Ziegler, Optimal bounds for a colorful Tverberg-Vrećica type problem, Adv. Math. 226 (2011), no. 6, 5198-5215, DOI 10.1016/j.aim.2011.01.009. MR2775898 446

[69] P. V. M. Blagojević, B. Matschke, and G. M. Ziegler, Optimal bounds for the colored Tverberg problem, J. Eur. Math. Soc. (JEMS) 17 (2015), no. 4, 739-754, DOI 10.4171/JEMS/516. MR 3336834 446

[70] P. V. M. Blagojević and P. Soberón, Thieves can make sandwiches, Bull. Lond. Math. Soc. 50 (2018), no. 1, 108-123, DOI 10.1112/blms.12109. MR.3778548 1458 
[71] P. V. M. Blagojević and G. M. Ziegler, Beyond the Borsuk-Ulam theorem: the topological Tverberg story, A journey through discrete mathematics, Springer, Cham, 2017, pp. 273-341. MR 3726602 416

[72] A. Blum, S. Har-Peled, and B. Raichel, Sparse approximation via generating point sets, Proceedings of the Twenty-Seventh Annual ACM-SIAM Symposium on Discrete Algorithms, ACM, New York, 2016, pp. 548-557, DOI 10.1137/1.9781611974331.ch40. MR3478416 1438

[73] E. Boros and Z. Füredi, The number of triangles covering the center of an $n$-set, Geom. Dedicata 17 (1984), no. 1, 69-77, DOI 10.1007/BF00181519. MR.771183 1489

[74] E. Boros and V. Gurvich, Perfect graphs are kernel solvable, Discrete Math. 159 (1996), no. 1-3, 35-55, DOI 10.1016/0012-365X(95)00096-F. MR1415280 1463

[75] K. Borsuk, On the imbedding of systems of compacta in simplicial complexes, Fund. Math. 35 (1948), 217-234, DOI 10.4064/fm-35-1-217-234. MR0028019 441

[76] S. Boyd and L. Vandenberghe, Convex optimization, Cambridge University Press, Cambridge, 2004. MR2061575 1434, 446 447 469 470

[77] S. J. Brams and A. D. Taylor, Fair division: From cake-cutting to dispute resolution, Cambridge University Press, Cambridge, 1996. MR1381896 1454

[78] S. J. Brams, M. A. Jones, and C. Klamler, N-person cake-cutting: there may be no perfect division, Amer. Math. Monthly 120 (2013), no. 1, 35-47, DOI 10.4169/amer.math.monthly.120.01.035. MR3007365 1448 454

[79] S. J. Brams, D. M. Kilgour, and C. Klamler, How to divide things fairly, Math. Mag. 88 (2015), no. 5, 338-348, DOI 10.4169/math.mag.88.5.338. MR3470684 448, 454

[80] S. Brazitikos, Quantitative Helly-type theorem for the diameter of convex sets, Discrete Comput. Geom. 57 (2017), no. 2, 494-505, DOI 10.1007/s00454-016-9840-0. MR.3602863 442

[81] H. Brönnimann and M. T. Goodrich, Almost optimal set covers in finite VC-dimension, Discrete Comput. Geom. 14 (1995), no. 4, 463-479, DOI 10.1007/BF02570718. ACM Symposium on Computational Geometry (Stony Brook, NY, 1994). MR.1360948 483

[82] R. A. Brualdi and H. J. Ryser, Combinatorial matrix theory, Encyclopedia of Mathematics and its Applications, vol. 39, Cambridge University Press, Cambridge, 1991. MR.1130611 1462

[83] W. Bruns, J. Gubeladze, M. Henk, A. Martin, and R. Weismantel, A counterexample to an integer analogue of Carathéodory's theorem, J. Reine Angew. Math. 510 (1999), 179-185, DOI 10.1515/crll.1999.045. MR.1696095 1437

[84] B. Bukh, A point in many triangles, Electron. J. Combin. 13 (2006), no. 1, Note 10, 3. MR 2240753 1489

[85] B. Bukh, J. Matoušek, and G. Nivasch, Lower bounds for weak epsilon-nets and stair-convexity, Israel J. Math. 182 (2011), 199-208, DOI 10.1007/s11856-011-0029-1. MR 2783971 483

[86] B. Bukh, J. Matoušek, and G. Nivasch, Stabbing simplices by points and flats, Discrete Comput. Geom. 43 (2010), no. 2, 321-338, DOI 10.1007/s00454-008-9124-4. MR.2579699 479

[87] G. Calafiore and M. C. Campi, Uncertain convex programs: randomized solutions and confidence levels, Math. Program. 102 (2005), no. 1, Ser. A, 25-46, DOI 10.1007/s10107-0030499-y. MR2115479 475 476

[88] G. C. Calafiore and M. C. Campi, The scenario approach to robust control design, IEEE Trans. Automat. Control 51 (2006), no. 5, 742-753, DOI 10.1109/TAC.2006.875041. MR2232597 475476

[89] C. Carathéodory, Über den Variabilitätsbereich der Koeffizienten von Potenzreihen, die gegebene Werte nicht annehmen (German), Math. Ann. 64 (1907), no. 1, 95-115, DOI 10.1007/BF01449883. MR.1511425 4434

[90] B. Carl, Inequalities of Bernstein-Jackson-type and the degree of compactness of operators in Banach spaces, Ann. Inst. Fourier (Grenoble) 35 (1985), no. 3, 79-118. MR810669 1438

[91] G. D. Chakerian and S. K. Stein, Some intersection properties of convex bodies, Proc. Amer. Math. Soc. 18 (1967), 109-112, DOI 10.2307/2035233. MR0206818 1487

[92] G. D. Chakerian, Intersection and covering properties of convex sets, Amer. Math. Monthly 76 (1969), 753-766, DOI 10.2307/2317863. MR0250193 1439 
[93] T. M. Chan, An optimal randomized algorithm for maximum Tukey depth, Proceedings of the Fifteenth Annual ACM-SIAM Symposium on Discrete Algorithms, ACM, New York, 2004, pp. 430-436. MR2291081 1488

[94] T. M. Chan, Improved deterministic algorithms for linear programming in low dimensions, Proceedings of the Twenty-Seventh Annual ACM-SIAM Symposium on Discrete Algorithms, ACM, New York, 2016, pp. 1213-1219, DOI 10.1137/1.9781611974331.ch84. MR3478460 1474

[95] T. M. Chan, E. Grant, J. Könemann, and M. Sharpe, Weighted capacitated, priority, and geometric set cover via improved quasi-uniform sampling, Proceedings of the Twenty-Third Annual ACM-SIAM Symposium on Discrete Algorithms, ACM, New York, 2012, pp. 15761585. MR 3205315 1482

[96] B. Chazelle, Cutting hyperplanes for divide-and-conquer, Discrete Comput. Geom. 9 (1993), no. 2, 145-158, DOI 10.1007/BF02189314. MR1194032 481

[97] B. Chazelle, The discrepancy method: Randomness and complexity, Cambridge University Press, Cambridge, 2000. MR 1779341 482

[98] B. Chazelle, H. Edelsbrunner, M. Grigni, L. Guibas, M. Sharir, and E. Welzl, Improved bounds on weak $\epsilon$-nets for convex sets, Proceedings ACM symposium on Theory of computing (STOC) (1993), pp. 495-504. 1482

[99] D. Chen, O. Devillers, J. Iacono, S. Langerman, and P. Morin, Oja centers and centers of gravity, Comput. Geom. 46 (2013), no. 2, 140-147, DOI 10.1016/j.comgeo.2012.04.004. MR2983943 1490 491

[100] P.-A. Chen, On the multichromatic number of s-stable Kneser graphs, J. Graph Theory 79 (2015), no. 3, 233-248. 1460

[101] P.-A. Chen, On the chromatic number of almost s-stable Kneser graphs, arXiv:1711.06621 (2017). 1460

[102] P.-A. Chen, On the multichromatic number of s-stable Kneser graphs, J. Graph Theory 79 (2015), no. 3, 233-248, DOI 10.1002/jgt.21826. MR3346141 4460

[103] X. Chen and X. Deng, On the complexity of $2 D$ discrete fixed point problem, Theoret. Comput. Sci. 410 (2009), no. 44, 4448-4456, DOI 10.1016/j.tcs.2009.07.052. MR2561571 1433

[104] X. Chen, X. Deng, and S.-H. Teng, Settling the complexity of computing two-player Nash equilibria, J. ACM 56 (2009), no. 3, Art. 14, 57, DOI 10.1145/1516512.1516516. MR2536129 4452

[105] O. Cheong, K. Mulmuley, and E. Ramos, Randomization and derandomization, Handbook of Discrete Comput. Geom. (J. E. Goodman, J. O'Rourke, and C. D. Tóth, Eds.), CRC Press LLC, 2016, to appear. 483

[106] S. R. Chestnut, R. Hildebrand, and R. Zenklusen, Sublinear bounds for a quantitative Doignon-Bell-Scarf theorem, SIAM J. Discrete Math. 32 (2018), no. 1, 352-371, DOI 10.1137/16M1100095. MR3757097 1442443

[107] S. J. Chung, NP-completeness of the linear complementarity problem, J. Optim. Theory Appl. 60 (1989), no. 3, 393-399, DOI 10.1007/BF00940344. MR993006 1452

[108] V. Chvátal, On the computational complexity of finding a kernel, Tech. Rep., Centre de Recherches Mathématiques, Université de Montréal, 1973. 1463

[109] K. L. Clarkson, New applications of random sampling in computational geometry, Discrete Comput. Geom. 2 (1987), no. 2, 195-222, DOI 10.1007/BF02187879. MR884226 440

[110] K. L. Clarkson, Las Vegas algorithms for linear and integer programming when the dimension is small, J. Assoc. Comput. Mach. 42 (1995), no. 2, 488-499, DOI 10.1145/201019.201036. MR 1409744 1473

[111] K. L. Clarkson, H. Edelsbrunner, L. J. Guibas, M. Sharir, and E. Welzl, Combinatorial complexity bounds for arrangements of curves and spheres, Discrete Comput. Geom. 5 (1990), no. 2, 99-160, DOI 10.1007/BF02187783. MR1032370 1480

[112] K. L. Clarkson, D. Eppstein, G. L. Miller, C. Sturtivant, and S.-H. Teng, Approximating center points with iterative Radon points, Internat. J. Comput. Geom. Appl. 6 (1996), no. 3, 357-377, DOI 10.1142/S021819599600023X. ACM Symposium on Computational Geometry (San Diego, CA, 1993). MR1409651 488

[113] K. L. Clarkson and P. W. Shor, Applications of random sampling in computational geometry. II, Discrete Comput. Geom. 4 (1989), no. 5, 387-421, DOI 10.1007/BF02187740. MR.1014736 1440 
[114] J. Cloutier, K. L. Nyman, and F. E. Su, Two-player envy-free multi-cake division, Math. Social Sci. 59 (2010), no. 1, 26-37, DOI 10.1016/j.mathsocsci.2009.09.002. MR2587345 1455

[115] W. Cook, J. Fonlupt, and A. Schrijver, An integer analogue of Carathéodory's theorem, J. Combin. Theory Ser. B 40 (1986), no. 1, 63-70, DOI 10.1016/0095-8956(86)90064-X. MR830593 437

[116] R. W. Cottle, J.-S. Pang, and R. E. Stone, The linear complementarity problem, Classics in Applied Mathematics, vol. 60, Society for Industrial and Applied Mathematics (SIAM), Philadelphia, PA, 2009. Corrected reprint of the 1992 original [ MR1150683]. MR3396730 1451

[117] K.-D. Crisman and M. A. Jones, Eds. The mathematics of decisions, elections, and games (2014), vol. 624 of Contemporary Mathematics, American Mathematical Society, Providence, RI. 1448

[118] W. H. Cunningham, Theoretical properties of the network simplex method, Math. Oper. Res. 4 (1979), no. 2, 196-208, DOI 10.1287/moor.4.2.196. MR543931 1473

[119] G. B. Dantzig, Constructive proof of the Min-Max theorem, Pacific J. Math. 6 (1956), 25-33. MR 0081807 453 467

[120] L. Danzer, B. Grünbaum, and V. Klee, Helly's theorem and its relatives, Proc. Sympos. Pure Math., Vol. VII, Amer. Math. Soc., Providence, R.I., 1963, pp. 101-180. MR0157289 416. 434 439

[121] C. Daskalakis, P. W. Goldberg, and C. H. Papadimitriou, The complexity of computing a Nash equilibrium, STOC'06: Proceedings of the 38th Annual ACM Symposium on Theory of Computing, ACM, New York, 2006, pp. 71-78, DOI 10.1145/1132516.1132527. MR2277132 1452

[122] R. S. Datta, Universality of Nash equilibria, Math. Oper. Res. 28 (2003), no. 3, 424-432, DOI 10.1287/moor.28.3.424.16397. MR.1997243 453

[123] M. de Berg, O. Cheong, M. van Kreveld, and M. Overmars, Computational geometry: Algorithms and applications, 3rd ed., Springer-Verlag, Berlin, 2008. MR2723879 1473

[124] J. A. De Loera, R. Hemmecke, and M. Köppe, Algebraic and geometric ideas in the theory of discrete optimization, MOS-SIAM Series on Optimization, vol. 14, Society for Industrial and Applied Mathematics (SIAM), Philadelphia, PA; Mathematical Optimization Society, Philadelphia, PA, 2013. MR3024570 437 465 466

[125] J. A. De Loera, T. Hogan, F. Meunier, and N. Mustafa, Integer and mixed integer Tverberg numbers, Proceedings European Congress of Computational Geometry (2018). 1443

[126] J. A. De Loera, T. A. Hogan, D. Oliveros, and D. Yang, Tverberg-Type Theorems with Trees and Cycles as (Nerve) Intersection Patterns, arXiv:1808.00551 (2018). 1445

[127] J. A. De Loera, R. N. La Haye, D. Rolnick, and P. Soberón, Quantitative combinatorial geometry for continuous parameters, Discrete Comput. Geom. 57 (2017), no. 2, 318-334, DOI 10.1007/s00454-016-9857-4. MR3602856 1442

[128] J. A. De Loera, R. N. La Haye, D. Oliveros, and E. Roldán-Pensado, Chance-constrained convex mixed-integer optimization and beyond: two sampling algorithms within $S$-optimization, J. Convex Anal. 25 (2018), no. 1, 201-218. MR3778498 1475 476

[129] J. A. De Loera, R. N. La Haye, D. Oliveros, and E. Roldán-Pensado, Helly numbers of algebraic subsets of $\mathbb{R}^{d}$ and an extension of Doignon's theorem, Adv. Geom. 17 (2017), no. 4, 473-482, DOI 10.1515/advgeom-2017-0028. MR3714450 1476

[130] J. A. De Loera, R. N. La Haye, D. Rolnick, and P. Soberón, Quantitative Tverberg theorems over lattices and other discrete sets, Discrete Comput. Geom. 58 (2017), no. 2, 435-448, DOI 10.1007/s00454-016-9858-3. MR3679944 443 446

[131] J. A. De Loera, E. Peterson, and F. E. Su, A polytopal generalization of Sperner's lemma, J. Combin. Theory Ser. A 100 (2002), no. 1, 1-26, DOI 10.1006/jcta.2002.3274. MR.1932067 4429

[132] J. A. De Loera, J. Rambau, and F. Santos, Triangulations: Structures for algorithms and applications, Algorithms and Computation in Mathematics, vol. 25, Springer-Verlag, Berlin, 2010. MR2743368 489

[133] M. de Longueville, A course in topological combinatorics, Universitext, Springer, New York, 2013. MR2976494 1420 422 429 430

[134] M. de Longueville and R. T. Živaljević, Splitting multidimensional necklaces, Adv. Math. 218 (2008), no. 3, 926-939, DOI 10.1016/j.aim.2008.02.003. MR2414326 1458 
[135] É. Colin de Verdière, G. Ginot, and X. Goaoc, Helly numbers of acyclic families, Adv. Math. 253 (2014), 163-193, DOI 10.1016/j.aim.2013.11.004. MR.3148550 1441

[136] D. de Werra, The combinatorics of timetabling, European J. Oper. Res. 96 (1997), no. 3, 504-513. 1458

[137] X. Deng, Z. Feng, and R. Kulkarni, Octahedral tucker is PPA-complete, Electronic Colloquium on Computational Complexity (ECCC) (2017), vol. 24, p. 118. 1433 458

[138] X. Deng, Q. Qi, and A. Saberi, Algorithmic solutions for envy-free cake cutting, Oper. Res. 60 (2012), no. 6, 1461-1476, DOI 10.1287/opre.1120.1116. MR3009176 1455

[139] A. Deza, S. Huang, T. Stephen, and T. Terlaky, Colourful simplicial depth, Discrete Comput. Geom. 35 (2006), no. 4, 597-615, DOI 10.1007/s00454-006-1233-3. MR.2225675 1436 489

[140] R. Diestel, Graph theory, 3rd ed., Graduate Texts in Mathematics, vol. 173, Springer-Verlag, Berlin, 2005. MR2159259 1441

[141] M. G. Dobbins, A point in a nd-polytope is the barycenter of $n$ points in its $d$-faces, Invent. Math. 199 (2015), no. 1, 287-292, DOI 10.1007/s00222-014-0523-2. MR3294963 1435

[142] C. T. J. Dodson and P. E. Parker, A user's guide to algebraic topology, Mathematics and its Applications, vol. 387, Kluwer Academic Publishers Group, Dordrecht, 1997. MR.1430097 1427

[143] J.-P. Doignon, Convexity in cristallographical lattices, J. Geometry 3 (1973), 71-85, DOI 10.1007/BF01949705. MR0387090 1439

[144] A. Dold, Simple proofs of some Borsuk-Ulam results, Proceedings of the Northwestern Homotopy Theory Conference (Evanston, Ill., 1982), Contemp. Math., vol. 19, Amer. Math.

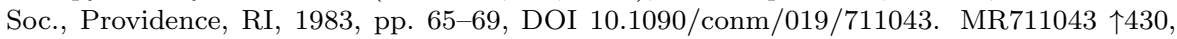
444

[145] V. Dol'nikov, Transversals of families of sets. Studies in the theory of functions of several real variables (Russian) (1981), 30-36. 1460

[146] L. E. Dubins and E. H. Spanier, How to cut a cake fairly, Amer. Math. Monthly 68 (1961), $1-17$, DOI 10.2307/2311357. MR0129031 1454

[147] P. Duchet, Graphes noyau-parfaits (French, with English summary), Ann. Discrete Math. 9 (1980), 93-101. Combinatorics 79 (Proc. Colloq., Univ. Montréal, Montreal, Que., 1979), Part II. MR597359 1463

[148] P. Duchet, Convexity in combinatorial structures, Proceedings of the 14th winter school on abstract analysis (Srní, 1986), Rend. Circ. Mat. Palermo (2) Suppl. 14 (1987), 261-293. MR 9208604434

[149] P. Duchet and H. Meyniel, Une généralisation du théorème de Richardson sur l'existence de noyaux dans les graphes orientés (French, with English summary), Discrete Math. 43 (1983), no. 1, 21-27, DOI 10.1016/0012-365X(83)90017-1. MR680300 1463

[150] A. Durand, M. Hermann, and L. Juban, On the complexity of recognizing the Hilbert basis of a linear Diophantine system, Theoret. Comput. Sci. 270 (2002), no. 1-2, 625-642, DOI 10.1016/S0304-3975(01)00017-2. MR.1871087 447 466 469

[151] J. Eckhoff, An upper-bound theorem for families of convex sets, Geom. Dedicata 19 (1985), no. 2, 217-227, DOI 10.1007/BF00181472. MR809468 1440

[152] J. Eckhoff, Helly, Radon, and Carathéodory type theorems, Handbook of convex geometry, Vol. A, B, North-Holland, Amsterdam, 1993, pp. 389-448. MR.1242986 1416 434

[153] J. Eckhoff, The partition conjecture, Discrete Math. 221 (2000), no. 1-3, 61-78, DOI 10.1016/S0012-365X(99)00386-6. Selected papers in honor of Ludwig Danzer. MR 1778908 1443

[154] J. Edmonds and R. Giles, A min-max relation for submodular functions on graphs, Studies in integer programming (Proc. Workshop, Bonn, 1975), North-Holland, Amsterdam, 1977, pp. 185-204. Ann. of Discrete Math., Vol. 1. MR0460169 1468

[155] F. Eisenbrand and G. Shmonin, Carathéodory bounds for integer cones, Oper. Res. Lett. 34 (2006), no. 5, 564-568, DOI 10.1016/j.orl.2005.09.008. MR.2238405 1437465

[156] P. Erdös, On sets of distances of $n$ points, Amer. Math. Monthly 53 (1946), 248-250, DOI 10.2307/2305092. MR0015796 1446 480

[157] J. Erickson, New lower bounds for Hopcroft's problem, Discrete Comput. Geom. 16 (1996), no. 4, 389-418, DOI 10.1007/BF02712875. Eleventh Annual Symposium on Computational Geometry (Vancouver, BC, 1995). MR1414963 1480

[158] K. Etessami and M. Yannakakis, On the complexity of Nash equilibria and other fixed points, SIAM J. Comput. 39 (2010), no. 6, 2531-2597, DOI 10.1137/080720826. MR2644357 1433 
[159] G. Even, D. Rawitz, and S. Shahar, Hitting sets when the VC-dimension is small, Inform. Process. Lett. 95 (2005), no. 2, 358-362, DOI 10.1016/j.ipl.2005.03.010. MR2149262 1483

[160] K. Fan, A generalization of Tucker's combinatorial lemma with topological applications, Ann. of Math. (2) 56 (1952), 431-437, DOI 10.2307/1969651. MR0051506 1425

[161] K. Fan, Simplicial maps from an orientable n-pseudomanifold into $S^{m}$ with the octahedral triangulation, J. Combinatorial Theory 2 (1967), 588-602. MR0214048 4430

[162] B. Farb, Group actions and Helly's theorem, Adv. Math. 222 (2009), no. 5, 1574-1588, DOI 10.1016/j.aim.2009.06.004. MR.2555905 1441

[163] A. Filos-Ratsikas and P. W. Goldberg, Consensus halving is PPA-complete, STOC'18Proceedings of the 50th Annual ACM SIGACT Symposium on Theory of Computing, ACM, New York, 2018, pp. 51-64. MR3826233 1458

[164] A. Filos-Ratsikas and P. W. Goldberg, The Complexity of Splitting Necklaces and Bisecting Ham Sandwiches, arXiv:1805.12559 (2018). 1458 478

[165] A. I. Flores, Über die Existenz n-dimensionaler Komplexe, die nicht in den $\mathbb{R}^{2 n}$ topologisch einbettbar sind, Ergeb. Math. Kolloqu. 5 (1933), 17-24. 1441

[166] J. Fox, M. Gromov, V. Lafforgue, A. Naor, and J. Pach, Overlap properties of geometric expanders, Proceedings ACM-SIAM Symposium on Discrete Algorithms (SODA) 2011, pp. 1188-1197. 1490

[167] R. M. Freund and M. J. Todd, A constructive proof of Tucker's combinatorial lemma, J. Combin. Theory Ser. A 30 (1981), no. 3, 321-325, DOI 10.1016/0097-3165(81)90027-3. MR618536 1433

[168] F. Frick, Counterexamples to the topological Tverberg conjecture, Oberwolfach Reports 12, (1) (2015), 318-321. 445

[169] F. Frick, Intersection patterns of finite sets and of convex sets, Proc. Amer. Math. Soc. 145 (2017), no. 7, 2827-2842, DOI 10.1090/proc/13443. MR3637933 1459

[170] F. Frick, Chromatic numbers of stable Kneser hypergraphs via topological Tverberg-type theorems, Int. Math. Res. Not. IMRN (2018). 1459, 460

[171] F. Frick, K. Houston-Edwards, and F. Meunier, Achieving rental harmony with a secretive roommate, Amer. Math. Monthly (to appear, 2017). 4456

[172] F. Frick, and S. Zerbib, Colorful coverings of polytopes and piercing numbers of colorful d-intervals, Combinatorica (to appear, 2018). 1436

[173] O. Friedmann, A subexponential lower bound for Zadeh's pivoting rule for solving linear programs and games, Integer programming and combinatorial optimization, Lecture Notes in Comput. Sci., vol. 6655, Springer, Heidelberg, 2011, pp. 192-206, DOI 10.1007/978-3-64220807-2_16. MR2820908 1473

[174] R. Fulek, A. F. Holmsen, and J. Pach, Intersecting convex sets by rays, Discrete Comput. Geom. 42 (2009), no. 3, 343-358, DOI 10.1007/s00454-009-9163-5. MR2519884 1490

[175] R. Fulek and J. Pach, A computational approach to Conway's thrackle conjecture, Comput. Geom. 44 (2011), no. 6-7, 345-355, DOI 10.1016/j.comgeo.2011.02.001. MR.2785903 1446

[176] D. Gale, The game of Hex and the Brouwer fixed-point theorem, Amer. Math. Monthly 86 (1979), no. 10, 818-827, DOI 10.2307/2320146. MR.551501 1418

[177] D. Gale, Equilibrium in a discrete exchange economy with money, Internat. J. Game Theory 13 (1984), no. 1, 61-64, DOI 10.1007/BF01769865. MR741586 1428

[178] H. Galeana-Sánchez and V. Neumann Lara, On kernels and semikernels of digraphs, Discrete Math. 48 (1984), no. 1, 67-76, DOI 10.1016/0012-365X(84)90131-6. MR732201 463

[179] A. Garber, On Helly number for crystals and cut-and-project sets, arXiv:1605.07881 (2016). 1476

[180] N. García-Colín, M. Raggi, and E. Roldán-Pensado, A note on the tolerant Tverberg theorem, Discrete Comput. Geom. 58 (2017), no. 3, 746-754, DOI 10.1007/s00454-017-9875-x. MR 3690670 1445

[181] J. Garg, R. Mehta, V. V. Vazirani, and S. Yazdanbod, ETR-completeness for decision versions of multi-player (symmetric) Nash equilibria, Automata, languages, and programming. Part I, Lecture Notes in Comput. Sci., vol. 9134, Springer, Heidelberg, 2015, pp. 554-566, DOI 10.1007/978-3-662-47672-7_45. MR3382467 1433 453

[182] B. Gärtner, J. Matoušek, L. Rüst, and P. Skovroň, Violator spaces: structure and algorithms, Discrete Appl. Math. 156 (2008), no. 11, 2124-2141, DOI 10.1016/j.dam.2007.08.048. MR 2437006 1475 
[183] P. Giannopoulos, M. Konzack, and W. Mulzer, Low-crossing spanning trees: an alternative proof and experiments, Proceedings European Workshop on Computational Geometry (2014). 484

[184] D. Gijswijt and G. Regts, Polyhedra with the integer Carathéodory property, J. Combin. Theory Ser. B 102 (2012), no. 1, 62-70, DOI 10.1016/j.jctb.2011.04.004. MR2871767 1437

[185] J. Gil, W. Steiger, and A. Wigderson, Geometric medians: Topological, algebraical and combinatorial structures, Discrete Math. 108 (1992), no. 1-3, 37-51, DOI 10.1016/0012365X(92)90658-3. Frolík's memorial volume. MR.1189827 1489

[186] I. Gilboa and E. Zemel, Nash and correlated equilibria: some complexity considerations, Games Econom. Behav. 1 (1989), no. 1, 80-93, DOI 10.1016/0899-8256(89)90006-7. MR.1000049 1451

[187] F. R. Giles and W. R. Pulleyblank, Total dual integrality and integer polyhedra, Linear Algebra Appl. 25 (1979), 191-196, DOI 10.1016/0024-3795(79)90018-1. MR528725 1468

[188] X. Goaoc, P. Paták, Z. Patáková, M. Tancer, and U. Wagner, Bounding Helly numbers via Betti numbers, A journey through discrete mathematics, Springer, Cham, 2017, pp. 407-447. MR.3726608 441

[189] M. X. Goemans and T. Rothvoß, Polynomiality for bin packing with a constant number of item types, Proceedings of the Twenty-Fifth Annual ACM-SIAM Symposium on Discrete Algorithms, ACM, New York, 2014, pp. 830-839, DOI 10.1137/1.9781611973402.61. MR3376421 465

[190] C. H. Goldberg and D. B. West, Bisection of circle colorings, SIAM J. Algebraic Discrete Methods 6 (1985), no. 1, 93-106, DOI 10.1137/0606010. MR772181 1457

[191] P. W. Goldberg and C. H. Papadimitriou, TFNP: an update, Algorithms and complexity, Lecture Notes in Comput. Sci., vol. 10236, Springer, Cham, 2017, pp. 3-9. MR 3657533 1433

[192] J. E. Graver, On the foundations of linear and integer linear programming. I, Math. Programming 9 (1975), no. 2, 207-226, DOI 10.1007/BF01681344. MR0386673 1466

[193] M. Gromov, Singularities, expanders and topology of maps. Part 2: From combinatorics to topology via algebraic isoperimetry, Geom. Funct. Anal. 20 (2010), no. 2, 416-526, DOI 10.1007/s00039-010-0073-8. MR2671284 489 493

[194] P. Gruber and J. Wills, Eds. Handbook of Convex Geometry. North-Holland, Amsterdam, 1993. 420

[195] P. M. Gruber, Convex and discrete geometry, Grundlehren der Mathematischen Wissenschaften [Fundamental Principles of Mathematical Sciences], vol. 336, Springer, Berlin, 2007. MR 2335496 1420

[196] L. Guth, Polynomial partitioning for a set of varieties, Math. Proc. Cambridge Philos. Soc. 159 (2015), no. 3, 459-469, DOI 10.1017/S0305004115000468. MR3413420 1485

[197] L. Guth, Polynomial methods in combinatorics, University Lecture Series, vol. 64, American Mathematical Society, Providence, RI, 2016. MR.3495952 4484

[198] L. Guth and N. H. Katz, On the Erdös distinct distances problem in the plane, Ann. of Math. (2) 181 (2015), no. 1, 155-190, DOI 10.4007/annals.2015.181.1.2. MR3272924 4484

[199] E. Győri, On division of graphs to connected subgraphs, Combinatorics (Proc. Fifth Hungarian Colloq., Keszthely, 1976), (1976), vol. 1, pp. 485-494. 1458

[200] G. Hahn and C. Tardif, Graph homomorphisms: structure and symmetry, Graph symmetry (Montreal, PQ, 1996), NATO Adv. Sci. Inst. Ser. C Math. Phys. Sci., vol. 497, Kluwer Acad. Publ., Dordrecht, 1997, pp. 107-166, DOI 10.1007/978-94-015-8937-6_4. MR1468789 1460. 461

[201] B. Hanke, R. Sanyal, C. Schultz, and G. M. Ziegler, Combinatorial Stokes formulas via minimal resolutions, J. Combin. Theory Ser. A 116 (2009), no. 2, 404-420, DOI 10.1016/j.jcta.2008.06.009. MR2475024 4430

[202] S. Har-Peled, Approximating Spanning Trees with Low Crossing Number, arXiv:0907.1131 (2009). 1484

[203] D. Haussler and E. Welzl, $\epsilon$-nets and simplex range queries, Discrete Comput. Geom. 2 (1987), no. 2, 127-151, DOI 10.1007/BF02187876. MR884223 4482

[204] P. E. Haxell, A condition for matchability in hypergraphs, Graphs Combin. 11 (1995), no. 3, 245-248, DOI 10.1007/BF01793010. MR1354745 461

[205] S. Hell, Tverberg-type theorems, and the Fractional Helly property, PhD thesis, T.U. Berlin, 2006. 1441 
[206] S. Hell, On the number of Tverberg partitions in the prime power case, European J. Combin. 28 (2007), no. 1, 347-355, DOI 10.1016/j.ejc.2005.06.005. MR2261824 1446

[207] S. Hell, On the number of Birch partitions, Discrete Comput. Geom. 40 (2008), no. 4, 586-594, DOI 10.1007/s00454-008-9083-9. MR2453329 1446

[208] A. J. Hoffman, Binding constraints and Helly numbers, Second International Conference on Combinatorial Mathematics (New York, 1978), Ann. New York Acad. Sci., vol. 319, New York Acad. Sci., New York, 1979, pp. 284-288. MR556036 1439440

[209] A. F. Holmsen, The intersection of a matroid and an oriented matroid, Adv. Math. 290 (2016), 1-14, DOI 10.1016/j.aim.2015.11.040. MR3451916 1435

[210] A. F. Holmsen, J. Pach, and H. Tverberg, Points surrounding the origin, Combinatorica 28 (2008), no. 6, 633-644, DOI 10.1007/s00493-008-2427-5. MR2488743 436

[211] A. F. Holmsen and R. Wenger, Helly-type theorems and geometric transversals, Handbook of discrete and computational geometry, CRC Press Ser. Discrete Math. Appl., CRC, Boca Raton, FL, 1997, pp. 63-82. MR 1730160 416 434

[212] A. F. Holmsen and R. Karasev, Colorful theorems for strong convexity, Proc. Amer. Math. Soc. 145 (2017), no. 6, 2713-2726, DOI 10.1090/proc/13405. MR 3626523 430 435

[213] A. F. Holmsen, M. Kim, and S. Lee, Nerves, minors, and piercing numbers, arXiv:1706.05181 (2017). 4442

[214] K. Houston-Edwards, Splitting rent with triangles, Infinite series, PBS digital studios February 23, 2017. https://www. youtube.com/watch?v=48oBEvpdYSE. 417

[215] S. Jadhav and A. Mukhopadhyay, Computing a centerpoint of a finite planar set of points in linear time, Discrete Comput. Geom. 12 (1994), no. 3, 291-312, DOI 10.1007/BF02574382. ACM Symposium on Computational Geometry (San Diego, CA, 1993). MR1298913 488

[216] J. Jonsson, On the chromatic number of generalized stable Kneser graphs, http://www . math.kth.se/ jakobj/doc/submitted/stablekneser.pdf (2012). 460

[217] G. Kalai, Intersection patterns of convex sets, Israel J. Math. 48 (1984), no. 2-3, 161-174, DOI 10.1007/BF02761162. MR.770699 1440

[218] G. Kalai, A subexponential randomized simplex algorithm, Proceedings ACM Symposium on Theory of computing (STOC) (1992), pp. 475-482. 1474

[219] G. Kalai, Combinatorics and convexity, Proceedings of the International Congress of Mathematicians, Vol. 1, 2 (Zürich, 1994), Birkhäuser, Basel, 1995, pp. 1363-1374. MR1404038 1416

[220] G. Kalai, Algebraic shifting, Computational commutative algebra and combinatorics (Osaka, 1999), Adv. Stud. Pure Math., vol. 33, Math. Soc. Japan, Tokyo, 2002, pp. 121-163. MR. 1890098 化4

[221] G. Kalai, Combinatorial expectations from commutative algebra, Combinatorial Commutative Algebra (I. Peeva and V. Welker, Eds.), vol. 1(3). Oberwolfach Reports, 2004, pp. 17291734. 1442

[222] G. Kalai and R. Meshulam, A topological colorful Helly theorem, Adv. Math. 191 (2005), no. 2, 305-311, DOI 10.1016/j.aim.2004.03.009. MR2103215 1430 435

[223] R. Kannan and T. Theobald, Games of fixed rank: a hierarchy of bimatrix games, Proceedings of the Eighteenth Annual ACM-SIAM Symposium on Discrete Algorithms, ACM, New York, 2007, pp. 1124-1132. MR2485264 1454

[224] R. N. Karasëv, Dual theorems on a central point and their generalizations (Russian, with Russian summary), Mat. Sb. 199 (2008), no. 10, 41-62, DOI 10.1070/SM2008v199n10ABEH003968; English transl., Sb. Math. 199 (2008), no. 9-10, 1459-1479. MR2473811 1491

[225] R. N. Karasëv, Topological methods in combinatorial geometry (Russian, with Russian summary), Uspekhi Mat. Nauk 63 (2008), no. 6(384), 39-90, DOI 10.1070/RM2008v063n06ABEH004578; English transl., Russian Math. Surveys 63 (2008), no. 6, 1031-1078. MR2492772 1422

[226] R. N. Karasev, Tverberg-type theorems for intersecting by rays, Discrete Comput. Geom. 45 (2011), no. 2, 340-347, DOI 10.1007/s00454-010-9294-8. MR2765534 4492

[227] R. Karasev, A simpler proof of the Boros-Füredi-Bárány-Pach-Gromov theorem, Discrete Comput. Geom. 47 (2012), no. 3, 492-495, DOI 10.1007/s00454-011-9332-1. MR2891243 489

[228] N. Karmarkar, A new polynomial-time algorithm for linear programming, Combinatorica 4 (1984), no. 4, 373-395, DOI 10.1007/BF02579150. MR.779900 1472 
[229] M. Katchalski and A. Liu, A problem of geometry in $\mathbf{R}^{n}$, Proc. Amer. Math. Soc. 75 (1979), no. 2, 284-288, DOI 10.2307/2042758. MR532152 1440

[230] D. C. Kay and E. W. Womble, Axiomatic convexity theory and relationships between the Carathéodory, Helly, and Radon numbers, Pacific J. Math. 38 (1971), 471-485. MR0310766 434

[231] L. G. Khachiyan, Polynomial algorithms in linear programming, USSR Computational Mathematics and Mathematical Physics 201 (1980), 53-72. 4472 476

[232] T. Király and J. Pap, A note on kernels and Sperner's lemma, Discrete Appl. Math. 157

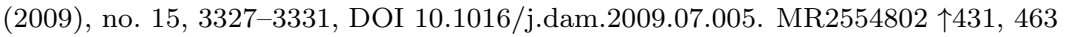

[233] V. Klee and G. J. Minty, How good is the simplex algorithm?, Inequalities, III (Proc. Third Sympos., Univ. California, Los Angeles, Calif., 1969; dedicated to the memory of Theodore S. Motzkin), Academic Press, New York, 1972, pp. 159-175. MR0332165 4773

[234] D. Kozlov, Combinatorial algebraic topology, Algorithms and Computation in Mathematics, vol. 21, Springer, Berlin, 2008. MR 2361455 1459

[235] D. Král, L. Mach, and J.-S. Sereni, A new lower bound based on Gromov's method of selecting heavily covered points, Discrete Comput. Geom. 48 (2012), no. 2, 487-498, DOI

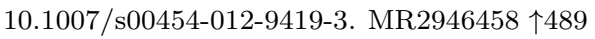

[236] M. A. Krasnosel'ski1, On a proof of Helly's theorem on sets of convex bodies with common points (Russian), Voronež. Gos. Univ. Trudy. Fiz.-Mat. Sb. 33 (1954), 19-20. MR0075615 1439

[237] S. Krishnan, N. H. Mustafa, and S. Venkatasubramanian, Hardware-assisted computation of depth contours, Proceedings ACM-SIAM Symposium on Discrete Algorithms (SODA) 2002, pp. 558-567. 488

[238] S. Krishnan, N. H. Mustafa, and S. Venkatasubramanian, Statistical data depth and the graphics hardware, Data depth: robust multivariate analysis, computational geometry and applications, DIMACS Ser. Discrete Math. Theoret. Comput. Sci., vol. 72, Amer. Math. Soc., Providence, RI, 2006, pp. 223-246. MR2343123 1489

[239] A. Kupavskii, N. H. Mustafa, and J. Pach, New lower bounds for $\epsilon$-nets, 32nd International Symposium on Computational Geometry, LIPIcs. Leibniz Int. Proc. Inform., vol. 51, Schloss Dagstuhl. Leibniz-Zent. Inform., Wadern, 2016, pp. Art. 54, 16. MR.3540896 1482

[240] S. Langerman and W. Steiger, The complexity of hyperplane depth in the plane, Discrete Comput. Geom. 30 (2003), no. 2, 299-309, DOI 10.1007/s00454-003-0011-x. U.S.-Hungarian Workshops on Discrete Geometry and Convexity (Budapest, 1999/Auburn, AL, 2000). MR2007967 1491

[241] N. Lebert, F. Meunier, and Q. Carbonneaux, Envy-free two-player m-cake and three-player two-cake divisions, Oper. Res. Lett. 41 (2013), no. 6, 607-610, DOI 10.1016/j.orl.2013.07.010. MR3131831 1455

[242] C. E. Lemke and J. T. Howson Jr., Equilibrium points of bimatrix games, J. Soc. Indust. Appl. Math. 12 (1964), 413-423. MR0173556 1451

[243] R. J. Lipton, E. Markakis, and A. Mehta, Playing large games using simple strategies, Proceedings ACM Conference on Electronic Commerce, (EC) (2003), pp. 36-41. 1454

[244] R. Y. Liu, On a notion of simplicial depth, Proc. Nat. Acad. Sci. U.S.A. 85 (1988), no. 6, 1732-1734, DOI 10.1073/pnas.85.6.1732. MR930658 砳8

[245] C.-Y. Lo, J. Matoušek, and W. Steiger, Algorithms for ham-sandwich cuts, Discrete Comput. Geom. 11 (1994), no. 4, 433-452, DOI 10.1007/BF02574017. MR.1273227 1478

[246] L. Lovász, Normal hypergraphs and the perfect graph conjecture, Discrete Math. 2 (1972), no. 3, 253-267, DOI 10.1016/0012-365X(72)90006-4. MR0302480 1464

[247] L. Lovász, Kneser's conjecture, chromatic number, and homotopy, J. Combin. Theory Ser. A 25 (1978), no. 3, 319-324, DOI 10.1016/0097-3165(78)90022-5. MR514625 1458

[248] L. Lovász, Graph minor theory, Bull. Amer. Math. Soc. (N.S.) 43 (2006), no. 1, 75-86, DOI 10.1090/S0273-0979-05-01088-8. MR/2188176 1458

[249] L. Lovász and M. D. Plummer, Matching theory, AMS Chelsea Publishing, Providence, RI, 2009. Corrected reprint of the 1986 original [MR0859549]. MR2536865 随2

[250] L. Lyusternik and L. Schnirel'mann, Méthodes topologiques dans les problèmes variationnels, Gosudarstvennoe Izdat. (1930); French transl., Actualités Sci. Indust., no. 118, Hermann, Paris, 1934. 4429

[251] M. Krein and D. Milman, On extreme points of regular convex sets (English with Ukrainian summary), Studia Math. 9 (1940), 133-138, DOI 10.4064/sm-9-1-133-138. MR0004990 4435 
[252] I. Mabillard and U. Wagner, Eliminating Tverberg points, I. An analogue of the Whitney trick, Computational geometry (SoCG'14), ACM, New York, 2014, pp. 171-180. MR.3382296 445

[253] L. Martínez-Sandoval, E. Roldán-Pensado, and N. Rubin, Further consequences of the colorful Helly hypothesis, 34th International Symposium on Computational Geometry, LIPIcs. Leibniz Int. Proc. Inform., vol. 99, Schloss Dagstuhl. Leibniz-Zent. Inform., Wadern, 2018, pp. Art. No. 59, 14. MR3824303 1436

[254] L. Martínez-Sandoval and R. Tamam, Depth with respect to a family of convex sets, arXiv:1612.03435 (2016). 487

[255] J. Matoušek, Efficient partition trees, Discrete Comput. Geom. 8 (1992), no. 3, 315-334, DOI 10.1007/BF02293051. ACM Symposium on Computational Geometry (North Conway,

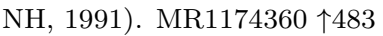

[256] J. Matoušek, Geometric Discrepancy: An Illustrated Guide, Springer, 1999. 1482

[257] J. Matoušek, Lectures on discrete geometry, Graduate Texts in Mathematics, vol. 212, Springer-Verlag, New York, 2002. MR.1899299 420, 439, 482,487

[258] J. Matoušek, Using the Borsuk-Ulam theorem: Lectures on topological methods in combinatorics and geometry, Universitext, Springer-Verlag, Berlin, 2003. Written in cooperation with Anders Björner and Günter M. Ziegler. MR1988723 1420 422 424 $425 \lcm{429} 441444$ 459, 478479

[259] J. Matoušek, A combinatorial proof of Kneser's conjecture, Combinatorica 24 (2004), no. 1, 163-170, DOI 10.1007/s00493-004-0011-1. MR2057690 1424 459

[260] J. Matoušek, Bounded VC-dimension implies a fractional Helly theorem, Discrete Comput. Geom. 31 (2004), no. 2, 251-255, DOI 10.1007/s00454-003-2859-z. MR2060639 1440

[261] J. Matoušek, Thirty-three miniatures: Mathematical and algorithmic applications of linear algebra, Student Mathematical Library, vol. 53, American Mathematical Society, Providence, RI, 2010. MR2656313 461

[262] J. Matoušek and B. Gärtner, Understanding and using linear programming, Springer Science \& Business Media, 2007. $450,464,465,471$

[263] J. Matoušek and G. M. Ziegler, Topological lower bounds for the chromatic number: a hierarchy, Jahresber. Deutsch. Math.-Verein. 106 (2004), no. 2, 71-90. MR2073516 1459

[264] J. Matoušek, M. Sharir, and E. Welzl, A subexponential bound for linear programming, Algorithmica 16 (1996), no. 4-5, 498-516, DOI 10.1007/s004539900062. MR1407586 1474

[265] J. Matoušek and U. Wagner, New constructions of weak $\epsilon$-nets, Discrete Comput. Geom. 32 (2004), no. 2, 195-206, DOI 10.1007/s00454-004-1116-4. MR2074337 低82

[266] J. Matoušek and U. Wagner, On Gromov's method of selecting heavily covered points, Discrete Comput. Geom. 52 (2014), no. 1, 1-33, DOI 10.1007/s00454-014-9584-7. MR.3231028 1489

[267] R. D. McKelvey and A. McLennan, Computation of equilibria in finite games, Handbook of computational economics, Vol. I, Handbooks in Econom., vol. 13, North-Holland, Amsterdam, 1996, pp. 87-142. MR.1416607 452

[268] A. McLennan, The expected number of Nash equilibria of a normal form game, Econometrica 73 (2005), no. 1, 141-174, DOI 10.1111/j.1468-0262.2005.00567.x. MR.2115633 1450

[269] A. McLennan and R. Tourky, Using volume to prove Sperner's lemma, Econom. Theory 35 (2008), no. 3, 593-597, DOI 10.1007/s00199-007-0257-0. MR2398817 1424

[270] R. Meshulam, The clique complex and hypergraph matching, Combinatorica 21 (2001), no. 1, 89-94, DOI 10.1007/s004930170006. MR1805715 430

[271] R. Meshulam, Domination numbers and homology, J. Combin. Theory Ser. A 102 (2003), no. 2, 321-330, DOI 10.1016/S0097-3165(03)00045-1. MR.1979537 1461

[272] F. Meunier, A $\mathbb{Z}_{q}$-Fan theorem, Tech. Rep., Laboratoire Leibniz-IMAG, Grenoble, 2005. 1430

[273] F. Meunier, Sperner labellings: a combinatorial approach, J. Combin. Theory Ser. A 113 (2006), no. 7, 1462-1475, DOI 10.1016/j.jcta.2006.01.006. MR2259071 垯9

[274] F. Meunier, The chromatic number of almost stable Kneser hypergraphs, J. Combin. Theory Ser. A 118 (2011), no. 6, 1820-1828, DOI 10.1016/j.jcta.2011.02.010. MR2793613 4457460

[275] F. Meunier and A. Deza, A further generalization of the colourful Carathéodory theorem, Discrete geometry and optimization, Fields Inst. Commun., vol. 69, Springer, New York, 2013, pp. 179-190, DOI 10.1007/978-3-319-00200-2_11. MR3156783 1436 
[276] F. Meunier, W. Mulzer, P. Sarrabezolles, and Y. Stein, The rainbow at the end of the line - a PPAD formulation of the colorful Carathéodory theorem with applications, Proceedings of the Twenty-Eighth Annual ACM-SIAM Symposium on Discrete Algorithms, SIAM, Philadelphia, PA, 2017, pp. 1342-1351, DOI 10.1137/1.9781611974782.87. MR.3627816 1447

[277] F. Meunier and P. Sarrabezolles, Colorful linear programming, Nash equilibrium, and pivots, Discrete Appl. Math. 240 (2018), 78-91, DOI 10.1016/j.dam.2016.10.006. MR3775040 1448

[278] F. Meunier and S. Zerbib Envy-free divisions of a partially burnt cake, arXiv:1804.00449 (2018). 1456

[279] G. L. Miller, and W. P. Thurston, Separators in two and three dimensions, Proceedings ACM Symposium on Theory of computing (STOC) (1990), pp. 300-309. 1487

[280] K. Miller, S. Ramaswami, P. Rousseeuw, T. Sellarès, D. Souvaine, I. Streinu, and A. Struyf, Fast implementation of depth contours using topological sweep, Proceedings of the Twelfth Annual ACM-SIAM Symposium on Discrete Algorithms (Washington, DC, 2001), SIAM, Philadelphia, PA, 2001, pp. 690-699. MR1958543 1488

[281] M. Mirzakhani and J. Vondrák, Sperner's colorings, hypergraph labeling problems and fair division, Proceedings of the Twenty-Sixth Annual ACM-SIAM Symposium on Discrete Algorithms, SIAM, Philadelphia, PA, 2015, pp. 873-886, DOI 10.1137/1.9781611973730.60. MR 3451084 1431

[282] M. Mirzakhani and J. Vondrák, Sperner's colorings and optimal partitioning of the simplex, A journey through discrete mathematics, Springer, Cham, 2017, pp. 615-631. MR.3726616 431

[283] I. Mizera, On depth and deep points: a calculus, Ann. Statist. 30 (2002), no. 6, 1681-1736, DOI 10.1214/aos/1043351254. MR.1969447 1491

[284] T. S. Motzkin, H. Raiffa, G. L. Thompson, and R. M. Thrall, The double description method, Contributions to the theory of games, vol. 2, Annals of Mathematics Studies, no. 28, Princeton University Press, Princeton, N. J., 1953, pp. 51-73. MR.0060202 421

[285] W. Mulzer and Y. Stein, Algorithms for tolerated Tverberg partitions, Algorithms and computation, Lecture Notes in Comput. Sci., vol. 8283, Springer, Heidelberg, 2013, pp. 295-305, DOI 10.1007/978-3-642-45030-328. MR3161009 1445

[286] W. Mulzer and Y. Stein, Computational aspects of the colorful Carathéodory theorem, 31st International Symposium on Computational Geometry, LIPIcs. Leibniz Int. Proc. Inform., vol. 34, Schloss Dagstuhl. Leibniz-Zent. Inform., Wadern, 2015, pp. 44-58. MR3392769 4448

[287] W. Mulzer and D. Werner, Approximating Tverberg points in linear time for any fixed dimension, Discrete Comput. Geom. 50 (2013), no. 2, 520-535, DOI 10.1007/s00454-0139528-7. MR 3090531 随8

[288] J. R. Munkres, Elements of algebraic topology, Addison-Wesley Publishing Company, Menlo Park, CA, 1984. MR755006 420 422

[289] O. R. Musin, Extensions of Sperner and Tucker's lemma for manifolds, J. Combin. Theory Ser. A 132 (2015), 172-187, DOI 10.1016/j.jcta.2014.12.001. MR3311342 1426 429

[290] O. R. Musin, Homotopy invariants of covers and KKM-type lemmas, Algebr. Geom. Topol. 16 (2016), no. 3, 1799-1812, DOI 10.2140/agt.2016.16.1799. MR.3523055 1429

[291] O. R. Musin, KKM type theorems with boundary conditions, J. Fixed Point Theory Appl. 19 (2017), no. 3, 2037-2049, DOI 10.1007/s11784-016-0388-7. MR.3692438 1428

[292] N. H. Mustafa, K. Dutta, and A. Ghosh, A Simple Proof of Optimal Epsilon-nets. Combinatorica (2017). https://doi.org/10.1007/s00493-017-3564-5. 1482

[293] N. H. Mustafa and S. Ray, Weak $\epsilon$-nets have basis of size $O(1 / \epsilon \log (1 / \epsilon))$ in any dimension, Comput. Geom. 40 (2008), no. 1, 84-91, DOI 10.1016/j.comgeo.2007.02.006. MR.2392655 1483

[294] N. H. Mustafa and S. Ray, An optimal extension of the centerpoint theorem, Comput. Geom. 42 (2009), no. 6-7, 505-510, DOI 10.1016/j.comgeo.2007.10.004. MR2519371 1483 487

[295] N. H. Mustafa and S. Ray, An optimal generalization of the colorful Carathéodory theorem, Discrete Math. 339 (2016), no. 4, 1300-1305, DOI 10.1016/j.disc.2015.11.019. MR 3442538 1436

[296] N. H. Mustafa, S. Ray, and M. Shabbir, Ray-shooting depth: computing statistical data depth of point sets in the plane, Algorithms - ESA 2011, Lecture Notes in Comput. Sci., vol. 6942, Springer, Heidelberg, 2011, pp. 506-517, DOI 10.1007/978-3-642-23719-5_43. MR.2893227 1490 
[297] N. H. Mustafa, S. Ray, and M. Shabbir, $k$-centerpoints conjectures for pointsets in $\mathbb{R}^{d}$, Internat. J. Comput. Geom. Appl. 25 (2015), no. 3, 163-185, DOI 10.1142/S0218195915500107. MR.3411828 4492

[298] N. H. Mustafa, H. R. Tiwary, and D. Werner, A proof of the Oja depth conjecture in the plane, Comput. Geom. 47 (2014), no. 6, 668-674, DOI 10.1016/j.comgeo.2013.12.006. MR.3168976 1491

[299] N. H. Mustafa and K. Varadarajan, Epsilon-approximations and epsilon-nets. Handbook of Discrete Comput. Geom. (J. E. Goodman, J. O'Rourke, and C. D. Tóth, Eds.), CRC Press LLC, 2017. 483

[300] J. F. Nash Jr., Equilibrium points in n-person games, Proc. Nat. Acad. Sci. U. S. A. 36 (1950), 48-49, DOI 10.1073/pnas.36.1.48. MR0031701 1450

[301] J. Nash, Non-cooperative games, Ann. of Math. (2) 54 (1951), 286-295, DOI 10.2307/1969529. MR0043432 450 452

[302] J. F. Nash, Jr., Some games and machines for playing them, Tech. Rep. D-1164, Rand Corporation, 1952. 418

[303] M. Naszódi, Proof of a conjecture of Bárány, Katchalski and Pach, Discrete Comput. Geom. 55 (2016), no. 1, 243-248, DOI 10.1007/s00454-015-9753-3. MR3439267 442

[304] N. Nisan, T. Roughgarden, E. Tardos, and V. V. Vazirani, Algorithmic Game Theory. Cambridge University Press, New York, NY, 2007. 1432 448,451

[305] A. Novikoff, On convergence proofs for perceptrons, Proc. Sympos. Math. Theory of Automata (New York, 1962), Polytechnic Press of Polytechnic Inst. of Brooklyn, Brooklyn, N.Y., 1963, pp. 615-622. MR.0175722 4438

[306] K. L. Nyman and F. E. Su, A Borsuk-Ulam equivalent that directly implies Sperner's lemma, Amer. Math. Monthly 120 (2013), no. 4, 346-354, DOI 10.4169/amer.math.monthly.120.04.346. MR3035127 1432

[307] H. Oja, Descriptive statistics for multivariate distributions, Statist. Probab. Lett. 1 (1983), no. 6, 327-332, DOI 10.1016/0167-7152(83)90054-8. MR721446 1490

[308] S. Onn, On the geometry and computational complexity of Radon partitions in the integer lattice, SIAM J. Discrete Math. 4 (1991), no. 3, 436-446, DOI 10.1137/0404039. MR1105949 4443

[309] S. Onn, Nonlinear discrete optimization: An algorithmic theory, Zurich Lectures in Advanced Mathematics, European Mathematical Society (EMS), Zürich, 2010. MR2724387 465,466

[310] M. Özaydin, Equivariant maps for the symmetric group (unpublished preprint), University of Winsconsin-Madison, 17 pages, available at http://digital.library.wisc.edu/1793/ 63829, 1987. 4444 445

[311] J. Pach and P. K. Agarwal, Combinatorial geometry, Wiley-Interscience Series in Discrete Mathematics and Optimization, John Wiley \& Sons, Inc., New York, 1995. A WileyInterscience Publication. MR 1354145482

[312] D. Pálvölgyi, 2d-Tucker is PPAD-complete, International Workshop on Internet and Network Economics (2009), Springer, pp. 569-574. 4433

[313] D. Pálvölgyi, Combinatorial necklace splitting, Electron. J. Combin. 16 (2009), no. 1, Research Paper 79, 8. MR 2529788 1457

[314] C. H. Papadimitriou, On the complexity of the parity argument and other inefficient proofs of existence, J. Comput. System Sci. 48 (1994), no. 3, 498-532, DOI 10.1016/S00220000(05)80063-7. 31st Annual Symposium on Foundations of Computer Science (FOCS) (St. Louis, MO, 1990). MR1279412 432 433457

[315] R. Pinchasi, A note on smaller fractional Helly numbers, Discrete Comput. Geom. 54 (2015), no. 3, 663-668, DOI 10.1007/s00454-015-9712-z. MR3392971 440

[316] G. Pisier, Remarques sur un résultat non publié de B. Maurey (French), Seminar on Functional Analysis, 1980-1981, École Polytech., Palaiseau, 1981, pp. Exp. No. V, 13. MR659306 1438

[317] A. Por, Universality of vector sequences and universality of Tverberg partitions, arXiv:1805.07197 (2018). 1445

[318] T. Prescott and F. E. Su, A constructive proof of Ky Fan's generalization of Tucker's lemma, J. Combin. Theory Ser. A 111 (2005), no. 2, 257-265, DOI 10.1016/j.jcta.2004.12.005. MR2156212 1426 432 
[319] M. Queyranne and F. Tardella, Carathéodory, Helly, and Radon numbers for sublattice and related convexities, Math. Oper. Res. 42 (2017), no. 2, 495-516, DOI 10.1287/moor.2016.0815. MR3652003 1476

[320] R. Rado, A theorem on general measure, J. London Math. Soc. 21 (1946), 291-300 (1947), DOI 10.1112/jlms/s1-21.4.291. MR.0021962 1487

[321] J. Radon, Mengen konvexer Körper, die einen gemeinsamen Punkt enthalten (German), Math. Ann. 83 (1921), no. 1-2, 113-115, DOI 10.1007/BF01464231. MR1512002 1442

[322] J. R. Reay, Generalizations of a theorem of Carathéodory, Mem. Amer. Math. Soc. No. 54 (1965), 50. MR0188891 434

[323] M. Richardson, Solutions of irreflexive relations, Ann. of Math. (2) 58 (1953), 573-590; errata 60 (1954), 595, DOI 10.2307/1969755. MR0075184 4463

[324] J. Robertson and W. Webb, Cake-cutting algorithms: Be fair if you can, A K Peters, Ltd., Natick, MA, 1998. MR1643406 1454

[325] D. Rolnick and P. Soberón, Algorithmic aspects of Tverberg's theorem, arXiv:1601.03083 (2016). 1448

[326] D. Rolnick and P. Soberón, Quantitative $(p, q)$ theorems in combinatorial geometry, Discrete Math. 340 (2017), no. 10, 2516-2527, DOI 10.1016/j.disc.2017.06.017. MR3674153 1442

[327] T. Ronkainen, H. Oja, and P. Orponen, Computation of the multivariate Oja median, Developments in robust statistics (Vorau, 2001), Physica, Heidelberg, 2003, pp. 344-359. MR.1977491 1491

[328] J. Rothe (ed.), Economics and computation: An introduction to algorithmic game theory, computational social choice, and fair division, Springer Texts in Business and Economics, Springer, Heidelberg, 2016. With illustrations by Irene Rothe. MR3381851 454

[329] J.-P. Roudneff, Partitions of points into simplices with $k$-dimensional intersection. $I$. The conic Tverberg's theorem, European J. Combin. 22 (2001), no. 5, 733-743, DOI 10.1006/eujc.2000.0493. Combinatorial geometries (Luminy, 1999). MR 1845497 1435 442

[330] P. Rousseeuw and I. Ruts, Algorithm AS 307: Bivariate location depth, J. R. Stat. Soc. Ser. C. Appl. Stat. 45 (1996), 516-526. 491

[331] P. J. Rousseeuw and M. Hubert, Regression depth, J. Amer. Statist. Assoc. 94 (1999), no. 446, 388-433, DOI $10.2307 / 2670155$. With discussion and a reply by the authors and Stefan Van Aelst. MR 1702314 1491

[332] N. Rubin, An improved bound for weak epsilon-nets in the plane, Proceedings IEEE Symposium on Foundations of Computer Science (FOCS) 2018. 1483

[333] H. J. Ryser, Neuere Probleme der Kombinatorik, Vorträge über Kombinatorik (July 1967), Oberwolfach, Mathematisches Forschunginstitute. 1462

[334] K. S. Sarkaria, Tverberg's theorem via number fields, Israel J. Math. 79 (1992), no. 2-3, 317-320, DOI 10.1007/BF02808223. MR.1248921 442

[335] K. S. Sarkaria, Tverberg partitions and Borsuk-Ulam theorems, Pacific J. Math. 196 (2000),

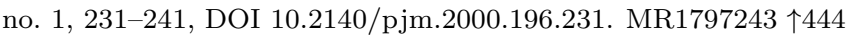

[336] P. Sarrabezolles, The colourful simplicial depth conjecture, J. Combin. Theory Ser. A 130 (2015), 119-128, DOI 10.1016/j.jcta.2014.11.002. MR 3280686 1489

[337] R. Savani and B. von Stengel, Exponentially many steps for finding a Nash equilibrium in a bimatrix game, Proceedings IEEE Symposium on Foundations of Computer Science (FOCS) 2004, pp. 258-267. 452

[338] H. Scarf, The approximation of fixed points of a continuous mapping, SIAM J. Appl. Math. 15 (1967), 1328-1343, DOI 10.1137/0115116. MR0242483 1432

[339] H. E. Scarf, The core of an $N$ person game, Econometrica 35 (1967), 50-69, DOI 10.2307/1909383. MR0234735 1431

[340] H. E. Scarf, An observation on the structure of production sets with indivisibilities, Proc. Nat. Acad. Sci. U.S.A. 74 (1977), no. 9, 3637-3641, DOI 10.1073/pnas.74.9.3637. MR 0452678 431 439

[341] M. Schaefer and D. Stefankovič, Fixed points, Nash equilibria, and the existential theory of the reals, Theory Comput. Syst. 60 (2017), no. 2, 172-193, DOI 10.1007/s00224-015-9662-0. MR.3600379 1433 453

[342] T. Schöneborn and G. M. Ziegler, The topological Tverberg theorem and winding numbers, J. Combin. Theory Ser. A 112 (2005), no. 1, 82-104, DOI 10.1016/j.jcta.2005.01.005. MR 2167476 化44 
[343] A. Schrijver, Vertex-critical subgraphs of Kneser graphs, Nieuw Arch. Wisk. (3) 26 (1978), no. 3, 454-461. MR512648 460

[344] A. Schrijver, Theory of linear and integer programming, Wiley-Interscience Series in Discrete Mathematics, John Wiley \& Sons, Ltd., Chichester, 1986. A Wiley-Interscience Publication. MR 874114 447 465 467 470

[345] A. Schrijver, Combinatorial Optimization: polyhedra and efficiency, Springer, 2003. 1420. 458,469

[346] T. Seacrest and F. E. Su, A lower bound technique for triangulations of simplotopes, SIAM J. Discrete Math. 32 (2018), no. 1, 1-28, DOI 10.1137/140972020. MR3740317 1450

[347] A. Sebő, Hilbert bases, Carathéodory's theorem and combinatorial optimization, Integer programming and combinatorial optimization (Waterloo, 1990), Univ. of Waterloo Press, 1990, pp. 431-455. 437

[348] E. Segal-Halevi, Fairly dividing a cake after some parts were burnt in the oven, arXiv:1704.00726 (2017). 1456

[349] R. Seidel, Small-dimensional linear programming and convex hulls made easy, Discrete Comput. Geom. 6 (1991), no. 5, 423-434, DOI 10.1007/BF02574699. MR.1115100 1473

[350] P. D. Seymour, Decomposition of regular matroids, J. Combin. Theory Ser. B 28 (1980), no. 3, 305-359, DOI 10.1016/0095-8956(80)90075-1. MR579077 4469

[351] A. Shapiro, D. Dentcheva, and A. Ruszczyński, Lectures on stochastic programming: Modeling and theory, 2nd ed., MOS-SIAM Series on Optimization, vol. 9, Society for Industrial and Applied Mathematics (SIAM), Philadelphia, PA; Mathematical Optimization Society, Philadelphia, PA, 2014. MR3242164 1475

[352] C. L. Siegel, Über einige Anwendungen diophantischer Approximationen, Abh. der Preus. Akad. der Wissenschaften. Phys.-math., 1 (1929), 209-266. 4437

[353] F. W. Simmons and F. E. Su, Consensus-halving via theorems of Borsuk-Ulam and Tucker, Math. Social Sci. 45 (2003), no. 1, 15-25, DOI 10.1016/S0165-4896(02)00087-2. MR.1963117 457

[354] S. Simon, Hyperplane equipartitions plus constraints, J. Combin. Theory Ser. A 161 (2019), 29-50, DOI 10.1016/j.jcta.2018.07.012. MR3861769 1479

[355] S. Smale, Mathematical problems for the next century, Math. Intelligencer 20 (1998), no. 2, 7-15, DOI 10.1007/BF03025291. MR1631413 473

[356] P. Soberón, Gerrymandering, sandwiches, and topology, Notices Amer. Math. Soc. 64 (2017), no. 9, 1010-1013, DOI 10.1090/noti1582. MR3699775 1454

[357] P. Soberón, Robust Tverberg and colourful Carathéodory results via random choice, Combin. Probab. Comput. 27 (2018), no. 3, 427-440, DOI 10.1017/S0963548317000591. MR3788169 445

[358] P. Soberón and R. Strausz, A generalisation of Tverberg's theorem, Discrete Comput. Geom. 47 (2012), no. 3, 455-460, DOI 10.1007/s00454-011-9379-z. MR2891241 445

[359] G. Spencer and F. E. Su, The LSB theorem implies the KKM lemma, Amer. Math. Monthly 114 (2007), no. 2, 156-159, DOI 10.1080/00029890.2007.11920401. MR2290367 1432

[360] S. Stahl, n-tuple colorings and associated graphs, J. Combinatorial Theory Ser. B 20 (1976), no. 2, 185-203. MR0406850 1461

[361] M. Stehlik, Personal communication. 1417

[362] S. K. Stein, Transversals of Latin squares and their generalizations, Pacific J. Math. 59 (1975), no. 2, 567-575. MR0387083 1462

[363] H. Steinhaus, Sur la division pragmatique (French), Econometrica 17 (1949), no. (Supplement), 315-319, DOI 10.2307/1907319. MR0039231 1454

[364] E. Steinitz, Bedingt konvergente Reihen und konvexe Systeme (German), J. Reine Angew. Math. 143 (1913), 128-176, DOI 10.1515/crll.1913.143.128. MR.1580879 1435

[365] A. H. Stone and J. W. Tukey, Generalized "sandwich" theorems, Duke Math. J. 9 (1942), 356-359. MR0007036 1479

[366] W. Stromquist, How to cut a cake fairly, Amer. Math. Monthly 87 (1980), no. 8, 640-644, DOI 10.2307/2320951. MR600922 1455

[367] W. Stromquist, Envy-free cake divisions cannot be found by finite protocols, Electron. J. Combin. 15 (2008), no. 1, Research Paper 11, 10. MR2368916 1455

[368] F. E. Su, Rental harmony: Sperner's lemma in fair division, Amer. Math. Monthly 106 (1999), no. 10, 930-942, DOI 10.2307/2589747. MR.1732499 1455 456 
[369] F. Su, A rectangular Sperner's lemma implies the Hex theorem, Working manuscript (2018). 455

[370] A. Sun, To divide the rent, start with a triangle, New York Times, April 29, 2014. https://www.nytimes.com/2014/04/29/science/to-divide-the-rent-start-witha-triangle.html?_r=1. 1417

[371] E. Szemerédi and W. T. Trotter Jr., Extremal problems in discrete geometry, Combinatorica 3 (1983), no. 3-4, 381-392, DOI 10.1007/BF02579194. MR729791 4480

[372] M. Tancer, Intersection patterns of convex sets via simplicial complexes: a survey, Thirty essays on geometric graph theory, Springer, New York, 2013, pp. 521-540, DOI 10.1007/9781-4614-0110-0_28. MR3205172 1441

[373] M. J. Todd, The number of necessary constraints in an integer program: a new proof of Scarf's theorem, Tech. Rep. 355, Cornell University, 1977. 1439

[374] M. J. Todd and L. Tunçel, A new triangulation for simplicial algorithms, SIAM J. Discrete Math. 6 (1993), no. 1, 167-180, DOI 10.1137/0406013. MR1201998 1432 450

[375] J. W. Tukey, Mathematics and the picturing of data, Proceedings of the International Congress of Mathematicians (Vancouver, B. C., 1974), Canad. Math. Congress, Montreal, Que., 1975, pp. 523-531. MR0426989 1487

[376] H. Tverberg, A generalization of Radon's theorem, J. London Math. Soc. 41 (1966), 123-128, DOI 10.1112/jlms/s1-41.1.123. MR0187147 1419 442

[377] H. Tverberg, A generalization of Radon's theorem. II, Bull. Austral. Math. Soc. 24 (1981), no. 3, 321-325, DOI 10.1017/S0004972700004858. MR647358 1435442

[378] H. Tverberg and S. Vrećica, On generalizations of Radon's theorem and the ham sandwich theorem, European J. Combin. 14 (1993), no. 3, 259-264, DOI 10.1006/eujc.1993.1029. MR 1215336 1435 442479

[379] J. D. Vaaler, The best constant in Siegel's lemma, Monatsh. Math. 140 (2003), no. 1, 71-89, DOI 10.1007/s00605-003-0047-0. MR2007141 1437

[380] M. L. J. van de Vel, Theory of convex structures, North-Holland Mathematical Library, vol. 50, North-Holland Publishing Co., Amsterdam, 1993. MR 1234493 1434

[381] E. R. van Kampen, Komplexe in euklidischen Räumen (German), Abh. Math. Sem. Univ. Hamburg 9 (1933), no. 1, 72-78, DOI 10.1007/BF02940628. MR3069580 441.

[382] M. van Kreveld, J. S. B. Mitchell, P. Rousseeuw, M. Sharir, J. Snoeyink, and B. Speckmann, Efficient algorithms for maximum regression depth, Discrete Comput. Geom. 39 (2008), no. 4, 656-677, DOI 10.1007/s00454-007-9046-6. MR2413152 1491

[383] V. N. Vapnik and A. Ja. Červonenkis, The uniform convergence of frequencies of the appearance of events to their probabilities (Russian, with English summary), Teor. Verojatnost. i Primenen. 16 (1971), 264-279. MR0288823 1481 482

[384] K. Varadarajan, Weighted geometric set cover via quasi-uniform sampling, STOC'10Proceedings of the 2010 ACM International Symposium on Theory of Computing, ACM, New York, 2010, pp. 641-647. MR.2743313 1482

[385] V. V. Vazirani, Approximation algorithms, Springer-Verlag, Berlin, 2001. MR.1851303 1465

[386] A. Yu. Volovikov, On a topological generalization of Tverberg's theorem (Russian), Mat. Zametki 59 (1996), no. 3, 454-456, DOI 10.1007/BF02308547; English transl., Math. Notes 59 (1996), no. 3-4, 324-325. MR 1399973 144

[387] J. von Neumann and O. Morgenstern, Theory of Games and Economic Behavior, Princeton University Press, Princeton, New Jersey, 1944. MR0011937 1463

[388] A. Vučić and R. T. Živaljević, Note on a conjecture of Sierksma, Discrete Comput. Geom.

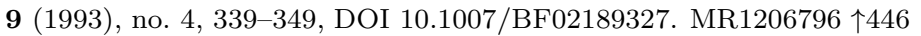

[389] U. Wagner, On $k$-Sets and Applications, PhD thesis, ETH Zurich, 2003. 1488

[390] M. Walter and K. Truemper, Implementation of a unimodularity test, Math. Program. Comput. 5 (2013), no. 1, 57-73, DOI 10.1007/s12532-012-0048-x. MR3030932 4469

[391] D. P. Williamson and D. B. Shmoys, The design of approximation algorithms, Cambridge University Press, Cambridge, 2011. MR2798112 1465

[392] D. R. Woodall, Dividing a cake fairly, J. Math. Anal. Appl. 78 (1980), no. 1, 233-247, DOI 10.1016/0022-247X(80)90225-5. MR.595779 1455 456

[393] A. C. Yao and F. F. Yao, A general approach to d-dimensional geometric queries, Proceedings ACM Symposium on Theory of Computing (STOC) (1985), pp. 163-168. 1479 
[394] X. Zhu, Recent developments in circular colouring of graphs, Topics in discrete mathematics, Algorithms Combin., vol. 26, Springer, Berlin, 2006, pp. 497-550, DOI 10.1007/3-540-337008_25. MR2249284 1460

[395] G. M. Ziegler, Generalized Kneser coloring theorems with combinatorial proofs, Invent. Math. 147 (2002), no. 3, 671-691, DOI 10.1007/s002220100188. MR 1893009 1424 457 459

[396] G. M. Ziegler, $3 N$ colored points in a plane, Notices Amer. Math. Soc. 58 (2011), no. 4, 550-557. MR2807521 1416

[397] R. T. Živaljević Oriented matroids and Ky Fan's theorem, Combinatorica 30, 4 (2010), 471-484. 1426

[398] R. T. Živaljević, Topological methods, Handbook of discrete and computational geometry, CRC Press Ser. Discrete Math. Appl., CRC, Boca Raton, FL, 1997, pp. 209-224. MR.1730167 1479

[399] R. T. Živaljević and S. T. Vrećica, The colored Tverberg's problem and complexes of injective functions, J. Combin. Theory Ser. A 61 (1992), no. 2, 309-318, DOI 10.1016/00973165(92)90028-S. MR1185000 446

University of California, Department of Mathematics, Davis, California 95616

Email address: deloera@math.ucdavis.edu

Université de Lorraine, CNRS, Inria, LORIA, F-54000 NANCy, France

Email address: xavier.goaoc@loria.fr

Université Paris Est, CERMiCS, ENPC, F-77454, Marne-la-Vallée, France

Email address: frederic.meunier@enpc.fr

Université Paris-Est, LiGM, Equipe A3Si, ESIEE Paris, Noisy le-Grand, France

Email address: nabilhassan.mustafa@esiee.fr 